\title{
Correlation between Thermal Transport Mechanisms and Microstructure of Epitaxially Grown $\left(\mathrm{Sb}_{1-\mathrm{x}} \mathrm{Bi}_{\mathrm{x}}\right)_{2} \mathrm{Te}_{3}$ Thin Films
}

\section{DissERTATION}

zur Erlangung des mathematisch-naturwissenschaftlichen Doktorgrades

\author{
"Doctor rerum naturalium" \\ der Georg-August-Universität Göttingen \\ im Promotionsprogramm ProPhys \\ der Georg-August University School of Science (GAUSS)
}

vorgelegt von

Felix Rieger

aus Stuttgart

Göttingen, 2019 


\section{Betreuungsausschuss:}

Prof. Dr. Christian Jooß, Institut für Materialphysik

Prof. Cynthia A. Volkert, Ph.D., Institut für Materialphysik

Prof. Dr. Vasily Moshnyaga, I. Physikalisches Institut

\section{Mitglieder der Prüfungskommission:}

Referent: Prof. Dr. Christian Jooß, Institut für Materialphysik

Korreferent: Prof. Dr. Vasily Moshnyaga, I. Physikalisches Institut

\section{Weitere Mitglieder der Prüfungskommission:}

Prof. Cynthia A. Volkert, Ph.D., Institut für Materialphysik

Prof. Dr. Michael Seibt, IV. Physikalisches Institut

Prof. Dr. Hans Christian Hofsäss, II. Physikalisches Institut

PD Dr. Martin Wenderoth, IV. Physikalisches Institut

Tag der mündlichen Prüfung: 26.04.2019 


\section{Contents}

1 Introduction $\quad 1$

1.1 Motivation . . . . . . . . . . . . . . . . . . . 1

2 Scientific background $\quad 5$

2.1 Thermoelectricity . . . . . . . . . . . . . . . . 5

2.1.1 Introduction and historical development . . . . . . . . . 5

2.1 .2 Origin of thermo voltages . . . . . . . . . . . 6

2.1.3 Efficiency of thermoelectric materials . . . . . . . . . . . 8

2.1.4 Currently used thermoelectric materials . . . . . . . . . . . . . . 10

2.2 Thermal conductivity in solid bodies . . . . . . . . . . . . 13

2.2 .1 Phonons . . . . . . . . . . . . . . . . . 13

2.2.2 Phonon modes and phononic dispersion relations . . . . . . . . . 13

2.2.3 Scattering mechanisms of phonons . . . . . . . . . . . . . 16

2.2.4 Temperature dependence of lattice thermal conductivity . . . . . . 19

$2.3 \quad\left(\mathrm{Sb}_{1-\mathrm{x}} \mathrm{Bi}_{\mathrm{x}}\right)_{2} \mathrm{Te}_{3}$ - a dedicated thermoelectric material system . . . . . . . . 21

2.3.1 Crystallographic structure and typical defects . . . . . . . . . . 21

2.3.2 Basic material properties . . . . . . . . . . . . . . . . . 23

2.3.3 Bonding and phonon dispersions . . . . . . . . . . . . . 25

2.3.4 Thermal conductivity . . . . . . . . . . . . . . . . 27

2.3.5 Kapitza resistances . . . . . . . . . . . . . . . . . . . 28

2.3.6 State of actual research on $\mathrm{Sb}_{2} \mathrm{Te}_{3}$ and $\mathrm{Bi}_{2} \mathrm{Te}_{3}$ thin films . . . . . 29

2.3.7 Superlattices and solid solutions . . . . . . . . . . . . . . . 34

3 Experimental techniques $\quad 37$

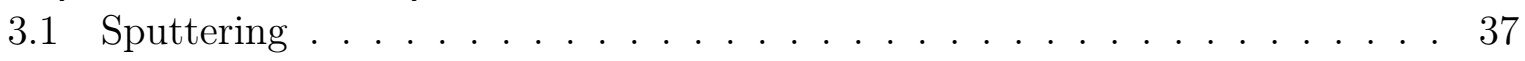

3.1 .1 Ion Beam Sputtering . . . . . . . . . . . . . . . . . 38

3.2 The $3 \omega$ method . . . . . . . . . . . . . . . . . . . . . . . . 40

3.2.1 Fundamentals . . . . . . . . . . . . . . . . . . . . . 40 40

3.2.2 The solution of the heat equation . . . . . . . . . . . . . . . . . . . . 41

3.2.3 The offset model . . . . . . . . . . . . . . . . . . . . . . . . . . . 43

3.2.4 Implementing the evaluation process . . . . . . . . . . . . . . . . . 45

3.2.5 The experimental setup . . . . . . . . . . . . . . 45 
3.3 TEM lamella preparation by Focused Ion Beam . . . . . . . . . . . . . . . 48

3.4 High Resolution Transmission Electron Microscopy . . . . . . . . . . . . . 49

4 Low intrinsic c-axis thermal conductivity in PVD grown epitaxial $\mathrm{Sb}_{2} \mathrm{Te}_{3}$ films

5 Transition into a phonon glass in crystalline thermoelectric $\left(\mathrm{Sb}_{1-\mathrm{x}} \mathrm{Bi}_{\mathrm{x}}\right)_{2} \mathrm{Te}_{3}$ films

6 General Discussion and Summary

Author contributions

Appendix 1: Low-temperature MOCVD deposition of $\mathrm{Bi}_{2} \mathrm{Te}_{3}$ thin films using $\mathrm{Et}_{2} \mathrm{BiTeEt}$ as single source precursor

Appendix 2: Structural and thermoelectrical characterization of epitaxial $\mathrm{Sb}_{2} \mathrm{Te}_{3}$ high quality thin films grown by thermal evaporation 


\section{CHAPTER 1}

\section{Introduction}

\subsection{Motivation}

Presently mankind is facing many global challenges. One of those big challenges is the man-made climate change. It is significantly caused by constantly rising $\mathrm{CO}_{2}$ emissions arising from burning fossil fuels in combustion machines and power plants. A rapidly increasing population worldwide, a higher standard of living for huge population groups and the inefficient use of energy will further lead to a conceivable rise of the global demand for energy. In view of these grand challenges, finding alternative routes of energy conversion and achieving higher efficiencies in using energy sources is of highest social interest.

The present work aspires to be a puzzle piece in this pursuit of knowledge and technological progress. It takes up current efforts on the more efficient use of energy and therefore focuses on so called thermoelectric materials. The potential applications of thermoelectric materials are numerous and promising. Utilization of waste heat in power plants and autarkic sensors in nature, technical systems and human body are only a few of those. Their unique strength consists in their capability of converting thermal into electrical energy without any moving parts. Thermoelectric devices are characterized by very low wear combined with exceptionally high reliability. However, there are challenges on their way to large-scale deployment. Mainly they comprise high material costs up to now and relatively modest efficiencies.

Already more than 100 years ago, the van der Waals (vdW) layered binary compounds $\mathrm{Bi}_{2} \mathrm{Te}_{3}$ and $\mathrm{Sb}_{2} \mathrm{Te}_{3}$ have been investigated with respect to thermoelectrical properties [1]. Since the middle of the 20th century, both compounds and the solid solution $\left(\mathrm{Sb}_{1-\mathrm{x}} \mathrm{Bi}_{\mathrm{x}}\right)_{2} \mathrm{Te}_{3}$ have been studied in more detail due to their high thermoelectric efficiency close to ambient temperature [2-6]. At the beginning, the main focus was on the development of high-performance Peltier elements for cooling applications.

Composition dependent measurements of electrical properties, thermal conductivity and thermoelectric effectivity performed at bulk samples prepared by the Bridgeman method revealed an optimum composition range between $\left(\mathrm{Sb}_{0.8} \mathrm{Bi}_{0.2}\right)_{2} \mathrm{Te}_{3}$ and $\left(\mathrm{Sb}_{0.6} \mathrm{Bi}_{0.4}\right)_{2} \mathrm{Te}_{3}[5]$. 
Since then, in the subsequent decades a lot of research has been done on this material system. Initially bulk samples have been investigated while later the investigation of thin films increased strongly. Since microstructure and doping play a major role in the semiconducting material $\left(\mathrm{Sb}_{1-\mathrm{x}} \mathrm{Bi}_{\mathrm{x}}\right)_{2} \mathrm{Te}_{3}$, the comparison of data obtained from differently synthesized samples is very challenging. A systematical temperature and composition dependent investigation of physical properties done at samples of the solid solution with well analyzed properties can hardly be found. Such data is crucial for a reliable assessment of structure-property correlations, however, many basic properties, such as the anisotropic thermal conductivity, are not well understood, even 100 years after first studies.

After the first studies at single crystals [5], the research shifted to nanostructuring approaches and multilayers, chasing a maximum value for the thermoelectric figure of merit $Z T$ at a specific temperature. Although that research efforts revealed a huge amount of interesting data, the interpretation was often not done on solid grounds. As questionable highlight of this development so far, a widely cited Nature publication by Venkatasubramanian et al. in 2001 [7] can be considered. The authors claimed a high $Z T$ value of 2.4 at $300 \mathrm{~K}$ for p-type $\mathrm{Sb}_{2} \mathrm{Te}_{3} / \mathrm{Bi}_{2} \mathrm{Te}_{3}$ superlattices synthesized by a MOCVD method. This outstanding value remains a "record" till today, since it could not be reproduced by other researchers. Nevertheless, this publication gave rise to enhanced worldwide research efforts on thermoelectric materials, presumably also against the background of climate change demanding for improved ways of power generation and consumption. To date, the reproduction of the high $Z T$ for superlattices failed, although great efforts have been made and different methods have been used as summarized by ref. [8] and ref. [9]. Also the reproducible synthesis of fully stable and thus useable nanostructures remains a big challenge, since thermoelectric materials in practice have to fulfill tough requirements with regard to thermodynamic and thermocycle stability.

With this work, a different path was taken: Epitaxially grown, nearly single crystalline $\left(\mathrm{Sb}_{1-\mathrm{x}} \mathrm{Bi}_{\mathrm{x}}\right)_{2} \mathrm{Te}_{3}$ thin films on $\alpha-\mathrm{Al}_{2} \mathrm{O}_{3}$ substrates, synthesized by a co-evaporation method, have been investigated with regard to correlation between structural and thermal properties. The [001] orientation of all films was confirmed by X-Ray Diffractometry (XRD) and topological analysis have been done by Scanning Electron Microscopy (SEM). Using a Focused Ion Beam (FIB) device, cross section lamellas have been prepared by a specially designed and very gentle procedure to avoid damage at the highly sensitive material. The lamellas have been studied extensively via High Resolution Transmission Electron Microscopy (HRTEM), Scanning Transmission Microscopy (STEM) and in particular by High Angle Annular Dark Field (HAADF) at an image corrected high performance TEM with lateral HRTEM resolution of $0.8 \AA$. Reaching atomic resolution, the substrate-film interface as well as defect types and densities were evaluated. While Energy Dispersive X-Ray Spectroscopy (EDX) gave global sample compositions, Electron Energy Loss Spectroscopy (EELS) at a $\mathrm{Sb}_{2} \mathrm{Te}_{3}$ film revealed the composition profile at the substrate-film interface and confirmed stoichiometric composition. Finally, by combining experimental and simulation results on STEM HAADF, the presence of solid 
solutions without pronounced agglomeration or depletion of any element has been shown.

A main goal of this thesis is the precise measurement of temperature and doping dependent $\mathrm{c}$-axis thermal conductivities of the $\left(\mathrm{Sb}_{1-\mathrm{x}} \mathrm{Bi}_{\mathrm{x}}\right)_{2} \mathrm{Te}_{3}$ films. This has been done by an advanced $3 \omega$ method. To obtain high-precision data, the obligatory subtraction of the insulation layer contribution has been done. Additionally, by appropriate layer thickness series, the interfacial thermal resistances (Kapitza resistances) occurring in the stack of layers have been determined. Thus a very precise measurement of thermal resistances and, derived from them, conductivities of films with different compositions has been successfully carried out over an extended temperature range.

Additional numerical calculations of the lattice thermal conductivities have been performed, implementing frequency and temperature dependent scattering mechanisms and using material specific properties. Matching the results with values gained from the $3 \omega$ measurements combined with literature data, revealed great insight into the impact of temperature, composition and phonon frequencies on phonon scattering mechanisms in $\left(\mathrm{Sb}_{1-\mathrm{x}} \mathrm{Bi}_{\mathrm{x}}\right)_{2} \mathrm{Te}_{3}$. For a composition with approximately every fourth $\mathrm{Sb}$ atom replaced by $\mathrm{Bi}$, the presence of a "Phonon glass - electron crystal" state over a remarkable temperature range has been shown.

This work grants a valuable dataset of conscientiously determined temperature dependent c-axis thermal conductivity of the ternary thermoelectric material $\left(\mathrm{Sb}_{1-\mathrm{x}} \mathrm{Bi}_{\mathrm{x}}\right)_{2} \mathrm{Te}_{3}$. It could shed light onto the intrinsic nature of the low thermal conductivity in these material systems which is due to both strong anharmonicity of the vdW bonds in c-direction and resonant bonds. It is present without any nanostructuring methods or high defect concentrations this material system possesses outstandingly low thermal conductivities, especially at certain doping levels. Furthermore, the structural analysis showed that the synthesis of highly textured thin films succeeded while using a scalable method, which makes the material even more attractive with regard to large-scale applications. Another important aspect is that presumably all findings about phonon scattering mechanisms to a great extend can be transferred to other vdW layered materials.

Finally, this thesis is a contribution on a wider field of materials science, since the method development concerning $3 \omega$ technique and data analysis can be applied at thin films composed of various other materials aiming at the understanding of heat transport mechanisms and microstructural properties. 



\section{CHAPTER 2}

\section{Scientific background}

\subsection{Thermoelectricity}

\subsubsection{Introduction and historical development}

Thermoelectric materials are a class of solid bodies, which are able to convert heat directly into electricity and vice versa. Physically thermoelectric devices are based on the Seebeck effect [10] respectively the Peltier effect [11]. There are two main applications:

- Thermoelectric Generators - TEGs: TEGs have been used for a long time in spacecrafts in so called Radioisotope Thermoelectric Generators (RTGs). Furthermore, thermoelectric materials are very interesting with regard to electric power generation from waste heat. Thermoelectrically usable waste heat is mainly created in power plants, industries, ships and cars, where up to now it is challenging to make it utilizable. Another application possibility consists in autarkic sensors e.g. in nature. TEGs integrated in clothes could use the body heat for driving small devices.

Last but not least, there are considerations of constructing implantable TEGs for recharging devices for pacemakers etc. This would overcome the need for regularly changing batteries. [12]

- Peltier elements: Peltier elements are for example used in solid state refridgerators. Here it is possible to cool down a volume without any moving parts and without any toxic liquid. The applications are manifold, e.g. for adjusting the temperature of cup holders and seats in cars.

Thermoelectric research had already taken place at the beginning of the 20th century by the German scientist E. Altenkirch [13,14], at the same time W. Haken mentioned the intermetallic Tellurium compounds $\mathrm{Sb}_{2} \mathrm{Te}_{3}$ and $\mathrm{Bi}_{2} \mathrm{Te}_{3}$ in the context of thermoelectricity [1]. This was taken up later again, for instance in 1947, when M. Telkes presented a large amount of intermetallic materials with high thermoelectric powers, including $\mathrm{Te}$ compounds such as Bismuth telluride [2]. Research on a large scale on intermetallic semiconductors started in the 1950s. Subsequently to the investigation of the binary 
compounds [3,4], the solid solution $\left(\mathrm{Sb}_{1-\mathrm{x}} \mathrm{Bi}_{\mathrm{x}}\right)_{2} \mathrm{Te}_{3}[5]$ has been studied in more detail. A pronounced Seebeck effect was also observed in Germanium [15] and Silicon [16], SiGe compounds are used for high temperature thermoelectric applications since decades. Many other compounds have been identified having optimum efficiency at different temperatures. At the end of the 1950s, thermoelectric research was an international effort, whereby one scientist who contributed a lot and should be expressly mentioned was the Sowjet physicist Abram Fjodorowitsch Joffe [17, 18].

For decades, the main application of thermoelectric materials have been RTGs in spacecrafts (and also for example in pacemakers and secluded lighthouses). They use the heat of a radioactive source such as for example ${ }^{238} \mathrm{Pu}\left({ }^{238} \mathrm{PuO}_{2}\right)$ or ${ }^{90} \mathrm{Sr}\left({ }^{90} \mathrm{SrTiO}_{3}\right)$. High temperatures can be reached and the thermal energy is converted into electricity with the help of TEGs.

There are some obstacles or challenges on the way to the widespread commercial use of TEGs. Bi-Te compounds and PbTe have a high density which makes devices heavy, a detriment especially for usage in cars. $\mathrm{Pb}$ compounds are additionally known for their toxicity which limits application and acceptance. The efficiency will be treated in subsection 2.1.3, one generally can say that TEGs have rather low to medium efficiencies. The temperature windows for high efficiency of a thermoelectric material usually is up to some hundred K. This leads to a segmented architecture in modern TEGs: Dependent on the temperature of their maximum figure of merit (see subsection 2.1.3) different materials are combined in a series [19]. By doing so and using high temperature gradients, it is possible to achieve decent efficiencies. RTGs with hot side temperature of $T_{H} \approx 1000{ }^{\circ} \mathrm{C}$ and temperature gradients of many $100 \mathrm{~K}$ at least reach $\eta$ in the upper single-digit percentage range, sufficient for the supply of several instruments on board of spacecrafts. However, even more important are high reliability and low degeneration rates.

\subsubsection{Origin of thermo voltages}

The Seebeck effect was first discovered by the Italian scientist Alessandro Volta in 1794 [20] and then rediscovered by its eponym, the Baltic German physicist Thomas Johann Seebeck in 1821 (presented in 1821 and 1822, publication printed in 1825 [10]). It describes the genesis of a voltage in a material between zones of different temperatures. This effect is used in TEGs to convert heat directly into electric energy without moving parts. Vice versa an applied voltage between the two ends of such a material creates a temperature gradient, based on the so called Peltier effect, which is used in Peltier elements, for example in solid state refrigerators.

In general, a temperature gradient between two sides of a material leads to a decrease of the chemical potential $\mu$ at the hot side which results in a so called thermo-diffusion current $j_{\mu}$ from the cold to the hot side. The hereby created electrical counter field $\phi$ leads to a drift-current $j_{d}$ of opposite sign. Both currents cancel each other out: 


$$
j_{\mu}+j_{d}=0
$$

Heat transport by electrons via $j_{d}$ takes place, but the effective transport of charge for open circuit conditions is 0 . A thermo voltage $U_{1}$ is created by a gradient in the chemical potential.

However, a second "type" of thermo voltages exists, caused by the Seebeck effect:

Electrons (respectively electron holes) at the hot side in average have higher kinetic energies, leading to a gradient $\nabla E_{k i n}$. As a result, at the hot side more electrons have energies above the chemical potential and their mobility is higher. This leads to a greater number of electrons drifting from the hot to the cold side than the other way round, resulting in a thermo-diffusion current from the hot to the cold side $j_{t h}$. The so created gradient in the electrical potential for its part generates a drift current $j_{d, t h}$ proportional to the gradient in the electric potential. At a certain point, a stationary state is reached:

$$
j_{t h}+j_{d, t h}=0
$$

In this stationary state, the currents cancel it other out, analogous to equation (2.1). The hereby created thermo voltage $U_{2}$ is of opposite sign to the thermo voltage $U_{1}$ caused by the gradient in the chemical potential and proportional to the temperature difference between hot and cold side $\Delta T$ :

$$
U_{2}=S \Delta T
$$

$S$ is the so called Seebeck coefficient, a crucial material specific key figure in thermoelectrics.

Consequently, a temperature gradient in a conducting material leads to an electric field:

$$
\overrightarrow{E_{t h}}=S \nabla T-\frac{1}{e} \nabla \mu=\left(S-\frac{1}{e} \frac{\partial \mu}{\partial T}\right) \nabla T
$$

To understand equation (2.4), it is important to take into account that $\nabla T$ and $\nabla \mu$ point into opposite directions and that $\frac{\partial \mu}{\partial T}$ is negative, since the chemical potential decreases with increasing temperature.

The size of the Seebeck coefficient $S$ depends on band structure, temperature, charge carrier densities and mobilities. Semiconductors with a Fermi level near the band gap are favorable since small changes in the kinetic energy have a huge impact. In particular, semiconductors with small band gaps provide good carrier concentrations, carrier mobilities and a huge temperature dependence of carrier mobilities. For that reason common thermoelectrics very often contain elements found in the 4th (Tetrels: $\mathrm{Si}, \mathrm{Ge}, \mathrm{Zn}, \mathrm{Pb}$ ), 5th (Pnictogens: P, As, Sb, Bi) and 6th (Chalkogens: O, S, Se, Te) main groups of the periodic table. The mentioned elements are the commonly used for thermoelectric applications from these groups. On the contrary, metals with broad bands are inappropriate 
for thermoelectric applications. Insulating materials do not show a Seebeck effect due to the non-existence of free charge carriers. The modulus of $S$ usually is between 0 and some hundred $\frac{\mathrm{\mu V}}{\mathrm{K}}$ for common materials, only in rare cases it exceeds $1 \frac{\mathrm{mV}}{\mathrm{K}}$ (e.g. $S=1.86 \frac{\mathrm{mV}}{\mathrm{K}}$ for amorphous $\mathrm{Pb}_{3} \mathrm{Ge}_{39} \mathrm{Se}_{48} \mathrm{Te}_{10}$ thin films [21]. It can take on negative and positive values depending on the nature of charge carriers present in the specific material (electrons respectively electron holes). Materials with both n- and p-conductivity are unfeasible because they exhibit two superimposing electric fields of different sign, canceling each other out to a certain degree and thus alleviating the Seebeck effect.

\subsubsection{Efficiency of thermoelectric materials}

Achieving high thermoelectric efficiencies is a non trivial problem, which is related to conflicting goals. To understand the challenges in this field of research, this subsection deals with the derivation and the physical interpretation of the mathematical formulas expressing that efficiency.

A derivation of these equations, which I want to outline (slightly modified) in this subsection, is given by G. D. Mahan [19].

Equations for electric and thermal currents (per area) are the starting point:

$$
\begin{gathered}
\vec{I}=-\sigma\left[-\nabla\left(\frac{\bar{\mu}}{e}\right)+S \nabla T\right] \\
\overrightarrow{I_{Q}}=T S \vec{I}-\kappa \nabla T
\end{gathered}
$$

Equation (2.5) builds up on equation (2.4): The first summand describes the electric current caused by the electrochemical potential $\bar{\mu}=\mu+e U$, the second summand the electric current caused by temperature gradients.

Equation (2.6) expresses the thermal current (heat flow) connected with the electric current. The thermal current caused by the temperature gradient (Fourier's law) is subtracted.

The subsequent derivation deals with a 1 dimensional problem due to $1 \mathrm{D}$ heat flow and gradient in the bars of thermoelectric generators or Peltier elements in steady-state mode. The efficiency is defined as the ratio of the developed power $P=I \frac{\Delta \bar{\mu}}{e}$ is and the heat drawn from the hot side $I_{Q, h}$ (both per area):

$$
\eta=\frac{P}{I_{Q, h}}
$$

Joule's heating caused by the electric current which is flowing through the device as it is in operation, is taken into account.

For the optimum efficiency with $\frac{\partial \eta}{\partial I}=0$ the final result is equation (2.8) expressing the efficiency $\eta$ in dependence of temperatures and material properties: 


$$
\begin{gathered}
\eta=\frac{T_{H}-T_{C}}{T_{H}} \frac{\sqrt{Z T+1}-1}{\sqrt{Z T+1}+\frac{T_{C}}{T_{H}}} \\
Z T=\frac{\sigma S^{2}}{\kappa} T=\frac{\sigma S^{2}}{\kappa_{l}+\kappa_{e}} T=\frac{\sigma S^{2}}{\kappa_{l}+L T \sigma} T
\end{gathered}
$$

The first fraction in equation (2.8) is the Carnot efficiency. It is lowered by the second fraction, containing the so called figure of merit (FOM) or $Z T$ (value). For $T$ usually the average of the two temperatures at the end of the bar is taken: $T=\frac{T_{H}+T_{C}}{2}$. However, since $Z$ is strongly $T$ dependent, one should calculate $\eta$ for many small temperature ranges subsequently adding the efficiencies up. The expression $\sigma S^{2}$ is also known as power factor $(\mathrm{PF})$.

As shown in equation (2.9) $Z T$ is material and strongly temperature dependent. For a huge $Z T$ value at first a huge Seebeck coefficient $S$ is needed. A good electrical conductivity $\sigma$ is beneficial as it stands in the numerator, but it is also part of the sum in the denominator since according to the Wiedeman-Franz law the electronic contribution to the thermal conductivity $\kappa_{e}$ is directly proportional to $\sigma$. However, one commonly aims at a high $\sigma$ since its positive impact prevails. Last but not least the lattice thermal conductivity $\kappa_{l}$ to has to be as low as possible. For that reason it is treated in detail in section 2.2 and a big part of the present thesis is about the precise measurement of thermal conductivities.

The charge carrier concentration $n$ influences $Z T$ strongly. It lowers the Seebeck coefficient $S$ and it increases the electronic and therefore also the thermal conductivity. The relationship between $n$ and $S$ for semiconductors and metals (approximation of energyindependent scattering, parabolic band) is [22]:

$$
S=\frac{8 \pi^{2} k_{B}^{2}}{3 e h} m^{*} T\left(\frac{\pi}{3 n}\right)^{\frac{2}{3}}
$$

$m^{*}$ is the effective mass of electrons or holes. As result the progression of $Z T(n)$ shows a maximum which lies for most materials in the range of $10^{19}$ to $10^{21} \frac{1}{\mathrm{~cm}^{3}}$. [22] Therefore doping plays a crucial role and thus the synthesis method of a material strongly influences its Seebeck coefficient. 


\subsubsection{Currently used thermoelectric materials}

There are some guidelines known for finding promising thermoelectric materials. Material properties beneficial for high thermoelectric performances are:

- A steep drop in the electronic DOS at or close to $\mathrm{E}_{\mathrm{F}}$ gives rise to a high Seebeck coefficient $S$.

- A narrow band gap is important for a high electrical conductivity $\sigma$ of the used semiconducting material.

- Heavy constituents are also helpful, especially for a low lattice thermal conductivity $\kappa_{l}$.

- A complex crystal structure further lowers $\kappa_{l}$ while it far less impacts the mobility of electrons.

- Saturated bondings, e.g. in $\mathrm{Si}, \mathrm{Ge}$ and $\mathrm{Si}-\mathrm{Ge}$ alloys. Those materials fulfill the so called „Octed rule".

- Different bonding types, e.g. covalent and van der Waals bonding. They induce high anharmonicity and lower the lattice thermal conductivity $\kappa_{l}$ by increased Umklapp scattering, see subsection 2.2.3. Van der Waals layered materials additionally show relatively low sound velocities due to the weak nature of that bonding type.

These are only the most common and important rules. A good thermoelectric material does not have to fulfill all those criteria but at least some.

It has to be noted that a high $Z T_{\max }$ value at a specific temperature is not expedient. More significant is the average $Z T$ in a specific temperature range and both high temperature and thermocycle stability. Reconciling all those considerations is a major challenge, including conflicts of objectives, which current research has to deal with.

A selection of used and/or promising material classes is given below, including common application temperatures and efficiencies:

- A common material system used around room temperature today is $\left(\mathrm{Sb}_{1-\mathrm{x}} \mathrm{Bi}_{\mathrm{x}}\right)_{2}\left(\mathrm{Te}_{1-\mathrm{y}} \mathrm{Se}_{\mathrm{y}}\right)_{3}$. It belongs to the class of Van der Waals layered thermoelectrics with resonant bondings within the quintuple layers. Known for many decades as an excellent thermoelectric material around room temperature $\left(Z T=1.86\right.$ at $320 \mathrm{~K}$ for nanostructured $\left(\mathrm{Sb}_{0.75} \mathrm{Bi}_{0.25}\right)_{2} \mathrm{Te}_{3}$ [23] $)$ its properties are still not fully understood and some important measurements have been skipped up to now. This was one of the reasons for the present study, where the quasi binary compound $\left(\mathrm{Sb}_{1-\mathrm{x}} \mathrm{Bi}_{\mathrm{x}}\right)_{2} \mathrm{Te}_{3}$ has been examined in detail.

In the last years, vdW layered thermoelectric materials with very high $Z T$ values at high temperatures came into focus of research. For the compound SnSe at $923 \mathrm{~K}$ a maximum value of $Z T=2.62$ along the b-axis and $Z T=2.3$ along the c-axis has been reported [24]. While these values of SnSe come along with an already low 
lattice thermal conductivity $\left(0.23 \frac{\mathrm{W}}{\mathrm{mK}}\right.$ at $\left.923 \mathrm{~K}\right)$, an ultra low thermal conductivity of $0.048 \frac{\mathrm{W}}{\mathrm{mK}}$ at $300 \mathrm{~K}$ was measured for a $62 \mathrm{~nm}$ thin film of vdW layered, disordered $\mathrm{WSe}_{2}[25]$.

- Oxides as for example $\mathrm{CaMnO}_{3}: \mathrm{Nb}, \mathrm{SrTiO}_{3}: \mathrm{Nb}$ and $\mathrm{Ca}_{3} \mathrm{Co}_{4} \mathrm{O}_{9}$ [26] respectively more complex layered systems as $\left(\mathrm{Ca}_{2} \mathrm{CoO}_{3}\right)_{0.62} \mathrm{CoO}_{2}$ [27] are promising thermoelectric materials for high temperature applications. Medium scale $Z T$ values can be reached, a rather high value for n-type thermoelectric oxides is $Z T=0.29$ at $1046 \mathrm{~K}$ for $\mathrm{Sr}_{0.9} \mathrm{La}_{0.1} \mathrm{TiO}_{3}$ ceramics [28]. The main advantages of oxide thermoelectrics reside in oxidation stability and low material costs.

- 4th main group element compounds: Silicon itself shows poor ZT values due to its high lattice thermal conductivity which is associated with the diamond structure. However, Si-Ge compounds show only moderate $\kappa_{l}$ and especially at high temperatures $S$ and $\sigma$ can be quite high. $\mathrm{Si}_{1-\mathrm{x}} \mathrm{Ge}_{\mathrm{x}}$ shows excellent high temperature stability and $Z T$ values (1.84 at $1073 \mathrm{~K}$ has been reported for nanostructured n-type $\mathrm{Si}_{0.8} \mathrm{Ge}_{0.2}$ [29]) and therefore it is used for high temperature applications, especially for RTGs in spacecrafts.

- Silicides: Compounds formed by transition group metals and Silicon are another promising material group for thermoelectric applications. Especially Magnesium and Manganese are interesting alloying elements due to their abundance in the earth crust, which is also an important benefit of Si itself. This abundance makes Silicides very cheap and balances well the fact, that their ZT values and efficiencies often range only midfield compared to other thermoelectrics. Their application temperatures are rather high and therefore higher Manganese Silicides (HMS: $\mathrm{Mn}_{4} \mathrm{Si}_{7}$, $\left.\mathrm{Mn}_{11} \mathrm{Si}_{18}, \mathrm{Mn}_{15} \mathrm{Si}_{26}, \mathrm{Mn}_{27} \mathrm{Si}_{47}\right)$ are promising TEG materials for waste heat utilization in industry. Especially the compound $\mathrm{Mn}_{15} \mathrm{Si}_{26}$ is of great interest, for spark plasma sintered (SPS) samples a maximum $Z T$ of 0.52 at $750 \mathrm{~K}$ was determined [30].

- Zintl phases, named after the German chemist Eduard Zintl (1898-1941), are intermetallic compounds formed out of alkali/alkaline earth metals (sometimes also Lanthanides) and elements between Group 11 (copper group) and Group 16 (Chalkogens). They show both ionic and covalent bondings and many of them are therefore semiconductors with relatively small band gaps. This makes some of them attractive for thermoelectric applications, especially those with huge unit cells as for example $\mathrm{Sr}_{21} \mathrm{Cd}_{4} \mathrm{Sb}_{18}$. Their good thermoelectric properties base on their special structure: For example the Zintl phase $\mathrm{Ca}_{\mathrm{x}} \mathrm{Yb}_{1-\mathrm{x}} \mathrm{Zn}_{2} \mathrm{Sb}_{2}$ consists out $\mathrm{Zn}_{2} \mathrm{Sb}_{2}^{2+}$ layers leading to electron-crystal properties. On the other side, Ca substitution by $\mathrm{Yb}$ atoms leads to tuning of the electron concentration and creates disorder for phonon-glass properties [31].

- Heusler compounds are named after the German chemist Friedrich Heusler (18661947) who in 1903 found the ferromagnetic alloy $\mathrm{Cu}_{2} \mathrm{MnAl}$ [32] consisting of non magnetic elements. Heusler compounds are magnetic intermetallic compounds with fcc crystal structure. To date more than 1000 compounds are known consisting of 
more than 40 chemical elements [33]. They are divided into full-Heuslers with composition $\mathrm{X}_{2} \mathrm{YZ}$ and half-Heuslers with composition XYZ. X and $\mathrm{Y}$ are transition metals respectively rare earth elements while $\mathrm{Z}$ represents a main group element. Several Heusler compounds exhibit properties beneficial for thermoelectricity, such as semiconductivity and topologically protected states. Additionally dopability is quite easy as e.g. the p-type $\mathrm{Fe}_{2} \mathrm{VAl}$ can be transferred to n-type by adding electrons in the form of replacing Fe atoms by Co atoms [34]. By substitution also enhanced Rayleigh scattering of phonons is realizable, e.g. in $\mathrm{Fe}_{16} \mathrm{~W}_{1} \mathrm{~V}_{7} \mathrm{Al}_{8}$, and the used elements mostly show moderate material costs. Complex structures give rise to good thermoelectric properties: For the half-Heusler compound $\mathrm{Ti}_{0.5} \mathrm{Zr}_{0.25} \mathrm{Hf}_{0.25} \mathrm{NiSn}_{0.98} \mathrm{Sb}_{0.02}$ (with an added densification aid) a $Z T_{\max }$ of 1.13 could be reached and for this compound an average value $Z T_{a v}$ of 0.74 has been measured in the temperature range from 300 to $825 \mathrm{~K}$ resulting in a thermoelectric efficiency of $\eta=11.8 \%$ [35].

- Skutterudites are a material class named after a naturally occurring mineral called Skutterudite with molecular formula $\mathrm{CoAs}_{3}$. What makes them interesting with regard to thermoelectric applications are voids in the crystal lattice, which can be filled which low coordinated ions, mostly those ions are rare earth metals. This leads to so called filled Skutterudites $\mathrm{R}_{\alpha} \mathrm{Y}_{4} \mathrm{Z}_{12}$, where $\mathrm{R}$ is an element of the lanthanides or actinides or a group 1 or group 2 element $(\alpha \leq 1)$, the position $\mathrm{Y}$ is occupied by one ore more transition metals and $\mathrm{Z}$ denotes one or more pniktogen elements, often $\mathrm{Sb}[36,37]$. These „rattling “ ions $\mathrm{R}$ give rise to strong scattering of, especially low frequency, phonons. $\kappa_{l}$ is thereby strongly decreased while $\sigma$ is only little influenced. Presumably, they will be applied in prospective RTGs in spacecrafts. A multiplicity of different compounds has been investigated, an extensive review has been written by Rull-Bravo et al. [38], including a detailed comparison with other thermoelectric materials. High figures of merit $Z T$ have been reported for different Skutterudites, reaching high values as e.g. 1.7 at $850 \mathrm{~K}$ for the compound $\mathrm{Ba}_{0.08} \mathrm{La}_{0.05} \mathrm{Yb}_{0.04} \mathrm{Co}_{4} \mathrm{Sb}_{12}$ [39].

- Intermetallic Clathrates are compounds containing a lattice that includes / traps other atoms (Latin clatratus: grilled). Different types are known, a common composition is $\mathrm{A}_{\mathrm{x}} \mathrm{B}_{\mathrm{y}} \mathrm{C}_{46-\mathrm{y}}$, where $\mathrm{A}$ is an alkali or alkaline earth metal (the so called "rattling" guest atom), while $\mathrm{B}$ and $\mathrm{C}$ are elements from main groups III respectively IV forming the cage. Often adopted stoichiometries are $\mathrm{A}_{8} \mathrm{~B}_{16} \mathrm{C}_{30}$ and $\mathrm{A}_{8} \mathrm{~B}_{8} \mathrm{C}_{38}$. The complex unit cell with the guest atom that effectively leads to strong scattering of phonons (comparable to Skutterudites) and unusual glasslike specific heats make Inorganic Clathrates a promising candidate for thermoelectric applications [37]. For $\mathrm{Ba}_{8} \mathrm{Ga}_{16} \mathrm{Ge}_{30}$ grown by the Czochralski method, a $Z T$ value of 1.35 at $900 \mathrm{~K}$ has been reported [40]. 


\subsection{Thermal conductivity in solid bodies}

As seen in the previous section, a key goal on the way to higher thermoelectric efficiencies is the reduction of thermal conductivity. The electronic contribution $\kappa_{c}=\sigma L T$ cannot be lowered without lowering the electrical conductivity $\sigma$, which affects the $Z T$ negatively. For that reason current thermoelectric research focuses on semiconductors with low phononic contributions to the thermal conductivity. Therefore this section is dedicated to the physical basics of lattice heat transport in solid bodies.

\subsubsection{Phonons}

The idea of the term "phonon" was first used by Jakow Illjitsch Frenkel in 1932 [41]. It was invented in analogy to the term "photon". Its philological origin is the Greek word $\phi \omega \nu \eta$ which can be translated to "sound". It is sometimes called a bosonic quasiparticle representing mechanical excitations in a periodic and elastic configuration of atoms respectively molecules. Hence this concept can only be applied in crystalline solids and some liquids.

\subsubsection{Phonon modes and phononic dispersion relations}

This subsection presents how energy and momentum of phonons are connected and shows the impact of this connection on material parameters such as the lattice thermal conductivity.

Phonons are bosons, so the expected number of phonon states is given by the BoseEinstein distribution:

$$
\langle n\rangle=\frac{1}{e^{\frac{\hbar \omega}{k^{T}}}-1}
$$

As phonons are basically the quanta of harmonic lattice oscillations, in first approximation the energy states are those of harmonic oscillators:

$$
E_{n}(k)=\hbar \omega(k)\left(n+\frac{1}{2}\right)
$$

The wave number $k$ is the modulus of the wave vector $\vec{k}$ and $\omega$ is the angular frequency of a phonon, while $n$ denotes the occupation number $(n=0,1,2, \ldots)$.

The relation $\omega(k)$ is called dispersion relation and represents the important connection between energy $E$ and momentum $\vec{p}$ of a phonon mode. 
In quantum mechanics, the following relationships apply:

$$
\begin{aligned}
& E=\hbar \omega \\
& \vec{p}=\hbar \vec{k}
\end{aligned}
$$

There are two different kinds of phonon modes: Acoustic and optical ones. The main difference between them is that in acoustic modes adjacent atoms oscillate cophasal while in optical modes they oscillate out of phase. One consequence is that optical modes only appear in crystals with a polyatomic basis, which is the case in the vast majority of materials. If adjacent atoms have different electric charges, the out of phase movement associated with optical modes can be excited by the electric field of a light wave, which is the origin of their nomenclature [42]. For example, in ionic crystals atoms with charges of opposite sign are caused to oscillate by infrared radiation.

A very important role dealing with thermal transport by phonons (lattice thermal conductivity $\kappa_{l}$ ) plays the ratio of phonon energy and phonon momentum, the so called dispersion relation. All considerations can be done within the first Brillouin zone because hereby all relevant wave numbers are covered. Let us assume a harmonic potential, a linear chain of atoms with a force constant $C$ between the atoms and a lattice parameter $a$.

Based on the assumption of a monoatomic (mass $M$ ) the motion equation (2.15) has to be solved where the force of neighbouring plains on plain $s$ is considered, $u$ is the respective spacial position [42].

$$
F_{s}=M \frac{d^{2} u}{d t^{2}}=C\left(u_{s+1}-u_{s}\right)+C\left(u_{s-1}-u_{s}\right)=C\left(u_{s+1}+u_{s-1}-2 u_{s}\right)
$$

Solving equation (2.15) leads to the phonon dispersion relation for acoustic phonon modes shown in (2.16) [42]:

$$
\omega_{a c}(k)=2 \sqrt{\frac{C}{M}}\left|\sin \left(\frac{k a}{2}\right)\right|
$$

For a diatomic basis (masses $M_{1}$ and $M_{2}$, positions $u$ and $v$ ) and based on the same force constant $C$ between the atoms, equation (2.17) and (2.18) have to be solved [42]:

$$
\begin{aligned}
& M_{1} \frac{d^{2} u_{s}}{d t^{2}}=C\left(v_{s}+v_{s-1}-2 u_{s}\right) \\
& M_{2} \frac{d^{2} v_{s}}{d t^{2}}=C\left(u_{s+1}+u_{s}-2 v_{s}\right)
\end{aligned}
$$

The exact solution in this case is more complicated than for only one type of atoms. Researching "real materials" one also has to deal with multiple force constants, different atomic distances and with atoms interacting which each other despite not being next neighbors. However, here a simple case is treated, which still reveals interesting basic 
information. In table 2.1 the approximated frequencies (for $M_{1}>M_{2}$ ) of the different phonon branches at the center and the border of the first Brillouin zone are presented. It is very important to notice the gap between acoustic $\left(\omega_{\max }=\sqrt{\frac{2 C}{M_{1}}}\right)$ and optical branch $\left(\omega_{\min }=\sqrt{\frac{2 C}{M_{2}}}\right)$, which is found for the vast majority of materials. Commonly there are also frequency gaps between different optical modes.

\begin{tabular}{|c|cc|}
\hline & $k=0$ & $k=\frac{\pi}{a}$ \\
\hline acoustic branch & 0 & $\sqrt{\frac{2 C}{M_{1}}}$ \\
optical branch & $\sqrt{2 C\left(\frac{1}{M_{1}}+\frac{1}{M_{2}}\right)}$ & $\sqrt{\frac{2 C}{M_{2}}}$ \\
\hline
\end{tabular}

Table 2.1: Phonon frequency limits of a linear lattice with two different types of atoms [42]

With regard to thermal conductivities, the group velocity $v_{g}$ of a wave package is a decisive factor. It represents the velocity of energy transport through the crystal. The group velocity of waves is the total derivative of the angular frequency with respective to the wave number:

$$
v_{g}=\frac{d \omega}{d k}
$$

On the basis of (2.16) the group velocities of acoustic modes can be calculated:

$$
\begin{gathered}
v_{g, a c}=\sqrt{\frac{C a^{2}}{M}} \cos \left(\frac{k a}{2}\right) \\
v_{g, o p} \ll v_{g, a c}
\end{gathered}
$$

As equation (2.21) implies, optical phonons can contribute to thermal conductivity. But though optical phonons generally exhibit higher frequencies and often cover huge frequency ranges, for thermal energy transport through the lattice, optical phonons usually play a minor role due to very small group velocities. Additionally, phonons with higher frequencies are stronger influenced by point defects which will be discussed in detail in the next subsection. And also a comparison of phonon dispersion relations of different materials and their massive effect on thermal material properties can be found in a later section. 


\subsubsection{Scattering mechanisms of phonons}

For understanding thermal properties of "real" materials the above introduced picture of phonons in a defect free crystal with an totally harmonic potential is not sufficient. Scattering of phonons at defects and other phonons plays a decisive role for the effective thermal conductivity of materials. In this subsection different scattering mechanisms will be discussed.

The phonon mean free path $l_{m f p}$ is a quantity which is lowered by scattering and thus limits the thermal conduction by phonons. In analogy to the Matthiessen rule used for the calculation of the total electrical resistivity in the presence of different electron scattering mechanisms, the total phonon mean free path can be determined by the formula $(2.22)$ out of the mean free paths resulting from the single scattering mechanisms:

$$
\frac{1}{l_{m f p}}=\frac{1}{l_{1}}+\frac{1}{l_{2}}+\frac{1}{l_{3}}+\ldots
$$

\section{Scattering of phonons grain boundaries}

In materials with small grains and/or low defect concentrations, at low temperatures the scattering at grain boundaries plays a role. In this case, the phonon mean free path $(2.23))$ is directly proportional to the average grain diameter $D_{\text {ave }}$ and often 1 is used as constant of proportionality [43].

$$
l_{g r} \propto D_{\text {ave }}
$$

The scattering at very rough surfaces is also known as Casimir limit [44], where only diffuse scattering takes place. In nanoscale systems it is possible that sample dimensions limit the phonon mean free path.

\section{Scattering of phonons at point defects}

Additional to grain boundary scattering, scattering at defects limits the maximum of the lattice thermal conductivity of a material. The scattering rate hereby depends on the defect density and the scattering cross sections. Since in good approximation it is not temperature dependent, it is often the dominating scattering mechanism at low temperatures.

The elastic scattering of photons at atoms and molecules is called Rayleigh scattering, named after the British physicist J. W. Strutt (3rd Baron Rayleigh), who presented it in ref. [45] and further publications. Since the rates of phonon scattering at point defects are also proportional to $\omega^{4}$ [46], this scattering mechanism is called Rayleigh scattering, too. The resulting mean free path can be expressed via equation (2.24), where B is a parameter which scales the strength of scattering, depending on sample specific properties.

$$
l_{R}=B\left(\frac{k_{B}}{\hbar \omega}\right)^{4}
$$


It is important to notice that phonons with high frequencies are much stronger scattered by point defects, which often lowers the contribution of optical phonons to heat transport processes in solid bodies. Actually, the frequency dependent contribution of phonons is strongly dependent on microstructure and defect density of a specific sample, which allows conclusions from thermal conductivity measurements.

\section{Phonon-phonon scattering}

In real crystals, the potential has anharmonic components. The potential is asymmetrical around the atomic position of rest due to the strong repulsive potential for small distances. These anharmonic components lead to phonon-phonon scattering. In three-phonon processes two phonons (momentums $\overrightarrow{p_{1}}$ and $\overrightarrow{p_{2}}$ ) interact with each other and transform into a third phonon with momentum $\overrightarrow{p_{3}}$. Also the splitting of one phonon into two is possible. Two different cases have to be distinguished:

- $\mathbf{N}$ process: Also called "normal process". The resulting momentum $\overrightarrow{p_{1}}+\overrightarrow{p_{2}}=\overrightarrow{p_{3}}$ lies within the first Brillouin zone, the phonon momentum is conserved. This process does not hinder the propagation of heat via phonons.

- U process: Also called "Umklapp process" or "Umklapp scattering", the concept and term was introduced by the German-born British physicist Rudolph Ernst Peierls in 1929 [47]. The resulting momentum $\overrightarrow{p_{1}}+\overrightarrow{p_{2}}$ "overtops" the first Brillouin zone and the wave vector of the resulting phonon is therefore turned down, which is the meaning of the German word "umklappen", which has negative impact on heat transport. The lattice transfers momentum $\vec{G}$, the reciprocal lattice vector, to the phonons: $\overrightarrow{p_{1}}+\overrightarrow{p_{2}}+\vec{G}=\overrightarrow{p_{3}}$. This is valid since the so called momentum of a phonon in fact is only a quasi momentum, defined in the first Brillouin zone, which means for a wave vector $\vec{k}: \vec{k}=\overrightarrow{k^{\prime}}+\vec{G}$.

$\mathrm{N}$ and $\mathrm{U}$ processes are also possible for the splitting of a single phonon into two phonons. One crucial requirement for Umklapp scattering of two phonons is that the sum of their momentums lies outside the first Brillouin zone. Additionally, energy and momentum of the involved phonons have to fit together, therefore the Umklapp scattering rates highly depend on phonon dispersion relations, frequencies and temperatures.

Different temperature dependences for Umklapp scattering rates have been found theoretically and experimentally for very low and very high temperatures [48, 49]. An overview is given by ref. [50].

The phonon mean free path progression in the medium temperature range (which depends mainly on $\Theta_{D}$ of the regarding material) can be expressed by equation (2.25). A is a coefficient determining the strength of Umklapp scattering, while $\left(\frac{k_{B}}{\hbar \omega}\right)^{2} \frac{1}{T}$ expresses the probability of scattering events in dependence of phonon frequencies and temperature. Additionally, the population of phonon modes is taken into account by the factor $\exp \left(\frac{\Theta_{D}}{\alpha T}\right)$ (coefficient $\alpha$ is related to material specific phonon properties, often $\alpha=3$ is used, see 
e.g. Supporting Information of ref. [27]) which approaches unity for high temperatures $T \gg \Theta_{D}$ leading to an $\frac{1}{T}$ behavior.

$$
l_{U}=A\left(\frac{k_{B}}{\hbar \omega}\right)^{2} \frac{1}{T} \exp \left(\frac{\Theta_{D}}{\alpha T}\right)
$$

In contrast to scattering at defects, Umklapp scattering is strongly temperature dependent. It reduces the phonon mean free path with rising temperature and thereby contributes to the temperature progression of the lattice thermal conductivity $\kappa_{l}(T)$. An elaborate treatise about the mathematical modelling of $\mathrm{U}$ and $\mathrm{N}$ processes is given by Han [51].

Higher orders of anharmonicity enable the possibility of four-phonon scattering. This includes the splitting of one phonon into three phonons as well as the scattering of two phonons generating two new phonons and the combination of three phonons into a single one. All three scenarios are possible as $\mathrm{N}$ respectively $\mathrm{U}$ process. In general, the transition probabilities of four-phonon processes are significantly low compared to three-phonon processes. But the scattering rates of four-phonon processes show a stronger dependence of temperatures and phonon frequencies compared to those of three-phonon processes. Theoretical calculations [52] reveal:

$$
\tau_{4}^{-1} \propto T^{2} \omega^{4}
$$

As a result, four-phonon $\mathrm{U}$ processes can be relevant for materials with high frequency phonons (and thus strong atomic bondings) at high temperatures far above room temperature. Feng et al. [52] showed a reduction of the predicted thermal conductivity at $1000 \mathrm{~K}$ of $30 \%$ for Silicon and Diamond and an even higher reduction of $60 \%$ for zinc-blende BAs, by considering four-phonon processes. In detail the exact impact of four-phonon processes is depending on transition probabilities of three- respectively four-phonon processes in the specific material. Equation (2.26) shows that high frequency phonons, higher frequency longitudinal acoustic (LA) phonons and optical phonons, can be significantly affected by four-phonon processes, but finally the material specific phonon dispersion relations determine the actual transition probabilities. Interestingly, deviations from the Debye model and a very low thermal conductivity observed for $\mathrm{Sb}_{2} \mathrm{Te}_{3}$, whereby higher-order anharmonic effects already at low temperatures are discussed as a possible explanation [53]. 


\subsubsection{Temperature dependence of lattice thermal conductivity}

To understand the temperature dependence of the lattice contribution to thermal conductivity, one has to combine the content discussed up to now. The thermal conductivity $\kappa$ at a specific temperature $T$ can be expressed by the equation (2.27):

$$
\kappa_{l}(T)=\sum_{n=1}^{N} \int_{\omega_{\min }}^{\omega_{\max }} g(\omega) C_{p h}(\omega, T) v_{n}(\omega) l_{m f p}(\omega, T) d \omega
$$

Equation (2.27) treats every single phonon mode separately (total number of modes $\mathrm{N})$ and integrates from the respective minimum angular frequency $\omega_{\min }$ to the respective maximum angular frequency $\omega_{\max } . g(\omega)$ is the phonon density of states at a specific angular frequency, $C_{p h}(\omega, T)$ denotes the specific heat capacity per single phonon and $v_{n}(\omega)$ is the mode specific and angular frequency dependent phonon velocity.

The specific heat shows a strong $T$ dependence, especially for low temperatures. Its frequency dependence is actually low in the relevant range. It can be obtained by differentiating the inner energy per phonon with respective to the temperature and is shown in equation (2.28).

$$
C_{p h}(\omega, T)=\frac{(\hbar \omega)^{2}}{k_{B} T^{2}} \frac{\exp \left(\frac{\hbar \omega}{k_{B} T}\right)}{\left(\exp \left(\frac{\hbar \omega}{k_{B} T}\right)-1\right)^{2}}
$$

Equation (2.29) represents a simple kinetic theory approximation. As phonon group velocity $v_{g}$, the average sound velocity is used. This is a good approximation since contributions by optical phonons are most often negligible. Additionally, an average mean free path of phonons $l_{m f p}(T)$ and a macroscopic specific heat capacity with respect to the volume $C_{V}(T)$ is used.

$$
\kappa_{l}(T)=\frac{1}{3} C_{V}(T) v_{g} l_{m f p}(T)
$$

Since phonons are treated individually in dependence of their frequencies and modes, equation (2.27) can reveal much more insights to scattering mechanisms and temperature and frequency dependences of phonon mean free paths.

However, for the sake of convenience, the temperature dependence of $\kappa_{l}$ will be discussed below on basis of equation (2.29), whereas equation (2.27) is the basis for the work presented in chapter 5 . The main trend will be illustrated with regard to the progressions of both specific heat capacity $C_{V}(T)$ and phonon mean free path $l_{m f p}(T)$. The phonon velocity is treated as a temperature-independent quantity.

In general, three different temperature regimes can be distinguished in crystals (for amorphous systems and the lower limit of disordered crystals see ref. [54]):

- $T \ll \Theta_{D}$ : For very low temperatures, Umklapp processes are negligible due to insufficient populated phonon modes and low phonon momentums. The mean free path can be very high, mainly depending on the microstructure (grain boundaries, point 
defects etc.), in extreme cases it is only limited by the sample dimensions. In this regime, the specific heat capacity $C_{V}(T)$ determines the temperature dependence of the lattice thermal conductivity and the Debye model yields: $\kappa_{l}(T) \propto T^{3}$

- Intermediate temperatures $T \approx \Theta_{D}$ : The most interesting temperature range is characterized by a maximum after the initial strong increase, followed by a decrease in $\kappa_{l}$. The position and height of the $\kappa_{l}$ peak is determined by anharmonicity, phonon dispersion relations, specific heat progression and defect concentrations. In this range, $C_{V}(T)$ is constantly rising, but with increasing temperature its slope is decreasing and thus $C_{V}(T)$ increasingly deviates from the $T^{3}$ behavior. On the other hand, phonon modes get more and more populated and Umklapp scattering increasingly influences the mean free path. Very general, the progression of $\kappa_{l}(T)$ therefore can be expressed (exponent $n$ decreases as $T$ rises) by: $\kappa_{l}(T) \propto T^{n} e^{\frac{\Theta}{\alpha T}}$

- $T \gg \Theta_{D}$ : For temperatures far above the Debye temperature $\Theta_{D}$ all phonon modes are populated and in good approximation, the specific heat can be considered to be constant. Thereby the mean free path determines the lattice thermal conductivity and the latter is dominated by the high temperature Umklapp scattering behavior: $\kappa_{l}(T) \propto \frac{1}{T}$

Phonon velocities are material specific property directly related to the phonon dispersion relations. Thus they are effected by the strength and nature of chemical bondings. On the other side, also the mean free path $l_{m f p}$ is material dependent, for example the atomic bondings determine the extend of anharmonicity. But additionally it is highly affected by defects and therefore $l_{m f p}$ is influenced by synthesis processes and by type and density of defects linked to it. 


\section{$2.3\left(\mathrm{Sb}_{1-\mathrm{x}} \mathrm{Bi}_{\mathrm{x}}\right)_{2} \mathrm{Te}_{3}-\mathrm{a}$ dedicated thermoelectric material system}

In this work, the binary intermetallic compound $\mathrm{Sb}_{2} \mathrm{Te}_{3}$ has been investigated. Furthermore, the $\mathrm{Sb}$ atoms have been replaced gradually by $\mathrm{Bi}$ atoms revealing the solid solution $\left(\mathrm{Sb}_{1-\mathrm{x}} \mathrm{Bi}_{\mathrm{x}}\right)_{2} \mathrm{Te}_{3}$ up to the binary compound $\mathrm{Bi}_{2} \mathrm{Te}_{3}$. In this section, the material properties of the binary compounds will be discussed and the state of actual research on $\left(\mathrm{Sb}_{1-\mathrm{x}} \mathrm{Bi}_{\mathrm{x}}\right)_{2} \mathrm{Te}_{3}$ as well as different synthesis methods are presented in order motivate the present thesis.

\subsubsection{Crystallographic structure and typical defects}

$\left(\mathrm{Sb}_{1-\mathrm{x}} \mathrm{Bi}_{\mathrm{x}}\right)_{2} \mathrm{Te}_{3}$ crystallizes in a trigonal crystal structure, its space group is $R \overline{3} m$, No.166, thus possessing the point group symmetry $D_{3 d}$. Therefore it has one 3 -fold rotation axis, three 2-fold rotation axes perpendicular to the 3 -fold rotation axis and three vertical mirror planes diagonally passing between the 2-fold rotation axes. Prototype of its crystal structure is the mineral Tetradymite with elemental formula $\mathrm{Bi}_{2} \mathrm{Te}_{2} \mathrm{~S}$. $\left(\mathrm{Sb}_{1-\mathrm{x}} \mathrm{Bi}_{\mathrm{x}}\right)_{2} \mathrm{Te}_{3}$ shows anisotropic behavior due to its layered structure: Five layers of atoms, perpendicular to the c-axis, form one so called quintuple layer (QL) and one unit cell consists of three of those quintuple layers in c-direction. The atomic sequence of one quintuple layer is $\mathrm{Te}(1)-\mathrm{Sb} / \mathrm{Bi}-\mathrm{Te}(2)-\mathrm{Sb} / \mathrm{Bi}-\mathrm{Te}(1)$. $\mathrm{Te}(1)$ and $\mathrm{Te}(2)$ differ with respective to the nature of their chemical bondings, therefore they are named differently. The main bonding type inside of a quintuple layer is covalent bonding while the quintuple layers only are connected with each other via van der Waals (vdW) forces [55]. This gives rise to anisotropy regarding different properties.

Due to the layered structure and a unit cell which is three quintupel layers high, common defects occurring in this layered material are stacking faults and the associated partial dislocations. Further common defects are twin boundaries, often realized by a rotation of $180^{\circ}$ around the c-axis, and antiphase boundaries with a shift of half a quintuple layer along the c-axis. Those planar defects can all be observed via High Resolution TEM respectively High Resolution STEM. Antisite defects, where a Pnictogen occupies a Te site or vice versa, are also very typical for this material.

Theoretical studies reveal antisite defect formation energies of 0.35 and $0.4 \mathrm{eV}$ for $\mathrm{Sb}_{2} \mathrm{Te}_{3}$ respectively $\mathrm{Bi}_{2} \mathrm{Te}_{3}$. The difference can be explained by different atomic radii and a small ionic bonding component being a little bit higher in case of $\mathrm{Bi}_{2} \mathrm{Te}_{3}$. [56] 


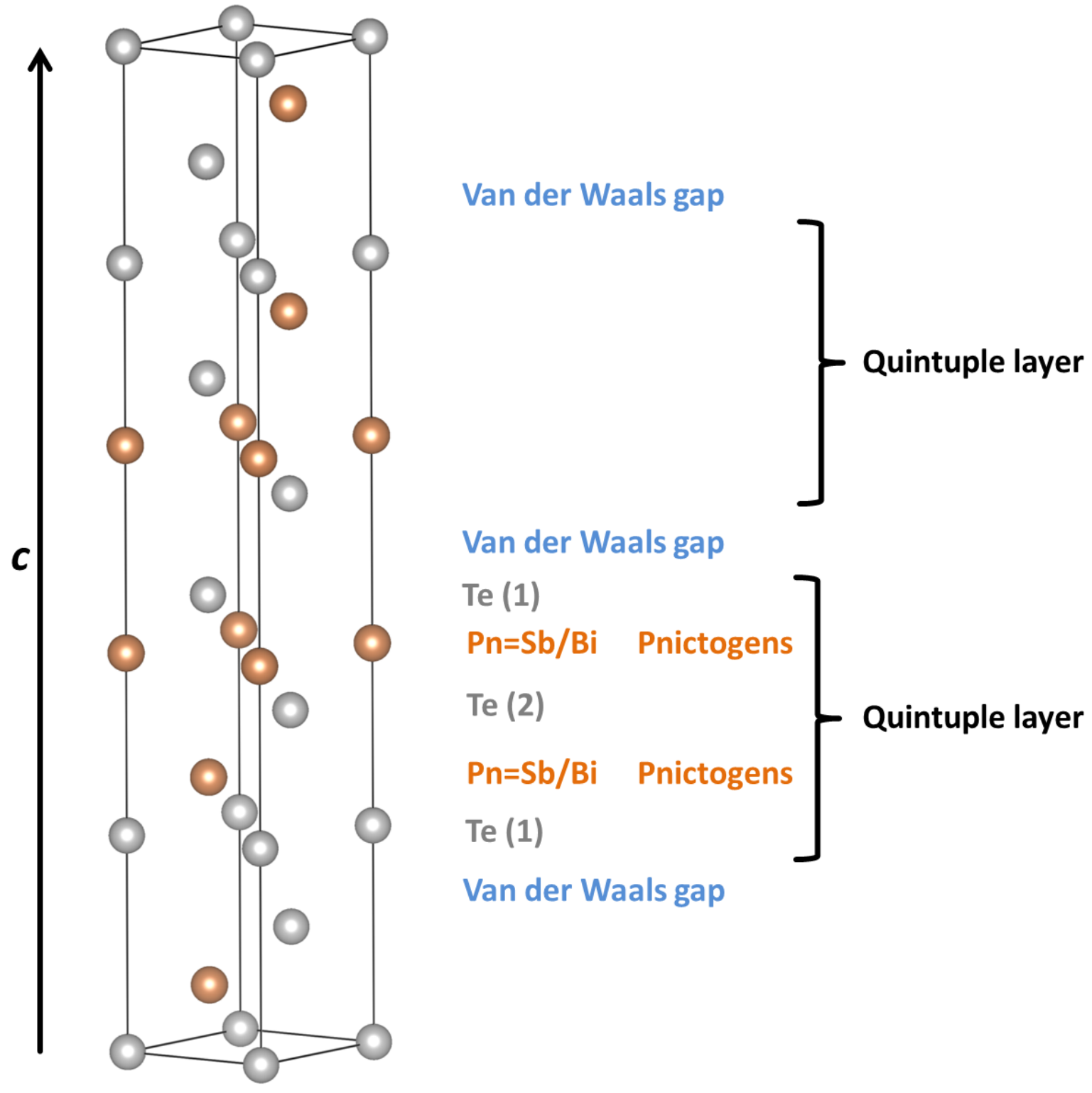

Figure 2.1: Unit cell of $\left(\mathrm{Sb}_{1-\mathrm{x}} \mathrm{Bi}_{\mathrm{x}}\right)_{2} \mathrm{Te}_{3}$ 


\begin{tabular}{|l|c|c|}
\hline Physical quantity & $\mathrm{Sb}_{2} \mathrm{Te}_{3}$ & $\mathrm{Bi}_{2} \mathrm{Te}_{3}$ \\
\hline Density $\rho\left[\frac{\mathrm{g}}{\mathrm{cm}^{3}}\right]$ & $6.50[57]$ & $7.74[57]$ \\
Melting temperature $T_{m}\left[{ }^{\circ} \mathrm{C}\right]$ & $620[57]$ & $580[57]$ \\
Lattice constant $a[\mathrm{~nm}]$ & $0.4262(1)[58]$ & $0.4395(3)[59]$ \\
Lattice constant $c[\mathrm{~nm}]$ & $3.0435(2)[58]$ & $3.044(1)[59]$ \\
Band gap $E_{g}[\mathrm{eV}]$ & $0.21[60]$ & $0.165[61]$ \\
Electrical conductivity $\sigma_{\perp c}\left[\frac{\mathrm{S}}{\mathrm{m}}\right]$ & $4.41 \times 10^{5}(289 \mathrm{~K})[62]$ & $5.30 \times 10^{4}(287 \mathrm{~K})[63]$ \\
Electrical conductivity $\sigma_{\| c}\left[\frac{\mathrm{S}}{\mathrm{m}}\right]$ & $1.79 \times 10^{5}(300 \mathrm{~K})[62]$ & $1.83 \times 10^{4}(287 \mathrm{~K})[63]$ \\
& & with $\eta_{\sigma}[64]$ \\
Thermal conductivity $\kappa_{\perp c}\left[\frac{\mathrm{W}}{\mathrm{mK}}\right]$ & $5.07[65]$ & $2.79[66]$ \\
Thermal conductivity $\kappa_{\| c}\left[\frac{\mathrm{W}}{\mathrm{mK}}\right]$ & $1.65 \pm 0.11[67]$ & $1.2 \pm 0.2[68]$ \\
Debye temperature $\Theta_{D}[\mathrm{~K}]$ & $165[69]$ & $165 \pm 2[70]$ \\
\hline
\end{tabular}

Table 2.2: Selected material properties of $\mathrm{Sb}_{2} \mathrm{Te}_{3}$ and $\mathrm{Bi}_{2} \mathrm{Te}_{3}$ at $T=300 \mathrm{~K}$ respectively the listed temperatures

\subsubsection{Basic material properties}

The binary compounds $\mathrm{Sb}_{2} \mathrm{Te}_{3}$ and $\mathrm{Bi}_{2} \mathrm{Te}_{3}$ have been investigated detailed and a lot of data is available on crystallography, electrical conductivities, band structures, Seebeck coefficients, preparation methods and thermal transport properties. Table 2.2 summarizes important basic material properties, the lattice constants are presented for the commonly used hexagonal crystal formalism.

For temperature dependent properties, if not stated differently, $300 \mathrm{~K}$ values have been chosen. Otherwise the measurement temperature is shown in brackets subsequent to the value.

Due to the anisotropy it is important to show some quantities perpendicular to the c-axis $(\perp c)$ respectively parallel to the c-axis $(\| c)$. The Seebeck coefficients $S$ are not shown due to their very strong dependence on doping and synthesis processes. However, in subsection 2.3.6 different methods for preparing thin films are presented often accompanied by determined values for $S$. 
$\mathrm{Sb}_{2} \mathrm{Te}_{3}$ and $\mathrm{Bi}_{2} \mathrm{Te}_{3}$ belong to the material class of so called topological insulators (TIs). These special materials show low bulk but much higher topological (viz. surface) electrical conductivity. They have been verified in 2007 by Laurens W. Molenkamp and coworkers [71] at HgTe quantum wells. In addition to conventional bulk states, topological insulators exhibit electronic surface states in between the band gap which lead to almost dissipationless charge transport. TIs are often systems with heavy elements and small band gaps. In such systems spin-orbit coupling can lead to band inversion at the surface. The band structure of the resulting topologically protected states is a metallic one. [72]

$\mathrm{Sb}_{2} \mathrm{Te}_{3}$ and $\mathrm{Bi}_{2} \mathrm{Te}_{3}$ have been identified to belong to the $\mathrm{Z}_{2}$ topological-insulator class by theoretical calculations [73]. There is experimental evidence for $\mathrm{Bi}_{2} \mathrm{Te}_{3}$ [61] and $\mathrm{Sb}_{2} \mathrm{Te}_{3}$ for being TIs [74].

First principle calculations done by Liang et al. [72] for $\mathrm{Bi}_{2} \mathrm{Te}_{3}$ predict only trivial surface states in films which are 1-2 quintuple layers thick. Films with thicknesses $\geq 3$ QLs show topologically protected states, also called Topologically Non-trivial Surface States (TNSS). Liang et al. found an indirect band gap of $304 / 61 \mathrm{meV}$ for the $1 / 2 \mathrm{QL}$ films while thicker films show metallic behavior. Bulk gaps of $170 / 140 / 135 / 128 \mathrm{meV}$ are reported for the $3 / 4 / 5 / 6$ QL films. [72]

Dealing with such TI systems one has to be careful with the expression „band gap“ since it obviously shows a strong thickness dependence. Ref. [72] addtionally predicts a high $Z T$ value for films with thicknesses in the range of the trivial - non trivial surface state transition due to a much higher relaxation time of the TNSS.

This is connected with the fact that topologically protected states show inhibited elastic backscattering generated by non-magnetic defects and impurities. On the other side, elastic scattering has to be taken into account since topological protection is closely linked to phase coherence which can be broken by inelastic scattering. [75]

A very important effect of TIs is the Anormalous Seebeck effect. The Seebeck effect usually is weak for metallic states for the already mentioned reasons, the energy dependence of the electron mean free path $l(E)$ normally is negligible. However, boundary-bulk interactions in TIs lead to a strong $l(E)$ dependency which is connected with a Seebeck coefficient $S$ much higher than those of conventional metals. [75]

Concerning thermoelectric performance this is especially interesting in combination with the high electrical conductivity of the topologically protected surface states.

Current thermoelectric research strives to utilize the high electrical conductivities at the surfaces of TIs. One approach is the manufacturing of materials with a high surface to volume ratio by creating grains in the range of some $\mathrm{nm}$. The idea behind is to increase the electrical conductivity while achieving increased phonon scattering at grain boundaries. This has been shown for $\mathrm{Bi}_{2} \mathrm{Se}_{3}$ bulk samples created by hot pressing of 
powder: Lowering the grain size from $300 \mathrm{~nm}$ to $80 \mathrm{~nm}$ decreased $\kappa$ by $30-50 \%$ and increased the charge carrier mobility by a factor of 50 . Between $100 \mathrm{~nm}$ and $80 \mathrm{~nm} \mathrm{a}$ transition from semiconducting to metallic $R(T)$ progression took place. [76]

However, while in case of $\mathrm{Bi}_{2} \mathrm{Se}_{3}$ in a specific range the resistivity can be decreased by decreasing the grain size, a work by Park et al. [77] showed for magnetron sputtered $\mathrm{Sb}_{2} \mathrm{Te}_{3}$ films with grain sizes between 200 and $350 \mathrm{~nm}$ the opposite behavior. The same result was achieved for sputtered $\left(\mathrm{Sb}_{0.1} \mathrm{Bi}_{0.9}\right)_{2} \mathrm{Te}_{3}$ films with grain sizes even beneath $100 \mathrm{~nm} \mathrm{[78]}$ and additionally the Seebeck coefficient was almost constant at approximately $-190 \frac{\mathrm{\mu V}}{\mathrm{K}}-$ 190. One can conclude that for the system $\left(\mathrm{Sb}_{1-\mathrm{x}} \mathrm{Bi}_{\mathrm{x}}\right)_{2} \mathrm{Te}_{3}$ the effect of such type of nano structuring seems to be limited, although more research has to be done on that field.

\subsubsection{Bonding and phonon dispersions}

The anharmonicity of Tetradymite type materials is high. One reason for that can be seen in the van der Waals bonded quintuple layers. Te(1) atoms are bonded covalently and by van der Waals forces which induces anharmonic potentials.

Additionally, within the quintuple layers, so called resonant bonding plays an important role:

In $\left(\mathrm{Sb}_{1-\mathrm{x}} \mathrm{Bi}_{\mathrm{x}}\right)_{2} \mathrm{Te}_{3}$, the Pnictogens (Sb respectively $\mathrm{Bi}$ ) exhibit three valence p-electrons while there are six available covalent bonds to their respective octahedral neighbors. The octahedral coordination leads to an alternation of the occupancy of the different available bonds. Important bases for the resonant bonding are furthermore a weak s-p hybridization as well as the fact that the s-orbital electrons form bands with much lower energies compared to the energies of the bands formed by p-orbital electrons. Since these bonds are strongly polarizable, this gives rise to distinct anharmonocity. Resonant bonds are not only beneficial in terms of low phonon thermal conductivities, but also by causing a high charge carrier mobility and thus relatively high electrical conductivities. [79]

The nature of atomic bondings in $\mathrm{Sb}_{2} \mathrm{Te}_{3}$ and $\mathrm{Bi}_{2} \mathrm{Te}_{3}$ is reflected in the phonon dispersion relations and their detailed analysis is worthwhile (see figures 2.2 and 2.3). The three lowest phonon modes in general are the three acoustic ones: Two transverse modes (TA) and one longitudinal mode (LA). The maximum frequencies of acoustic phonons (acoustic cutoff frequencies) of $\mathrm{Sb}_{2} \mathrm{Te}_{3}$ are $\mathrm{SI} 1.23 \mathrm{THz}$ parallel and $\mathrm{SI} 2.00 \mathrm{THz}$ perpendicular to the c-axis [80]. This demonstrates once more the anisotropic behavior of the investigated materials.

Along the c-axis, for both compounds the highest optical frequency is around four times higher than the maximum acoustic frequency. As a result, long before reaching the actual Debye temperature $\Theta_{D}$, the acoustic phonon modes are already populated. An acoustic Debye temperature $\Theta_{D, a c}$ can be introduced where all acoustic phonon modes are populated, values reported by ref. [81] for $\mathrm{Sb}_{2} \mathrm{Te}_{3}$ and $\mathrm{Bi}_{2} \mathrm{Te}_{3}$ are 94 respectively $71 \mathrm{~K}$. 


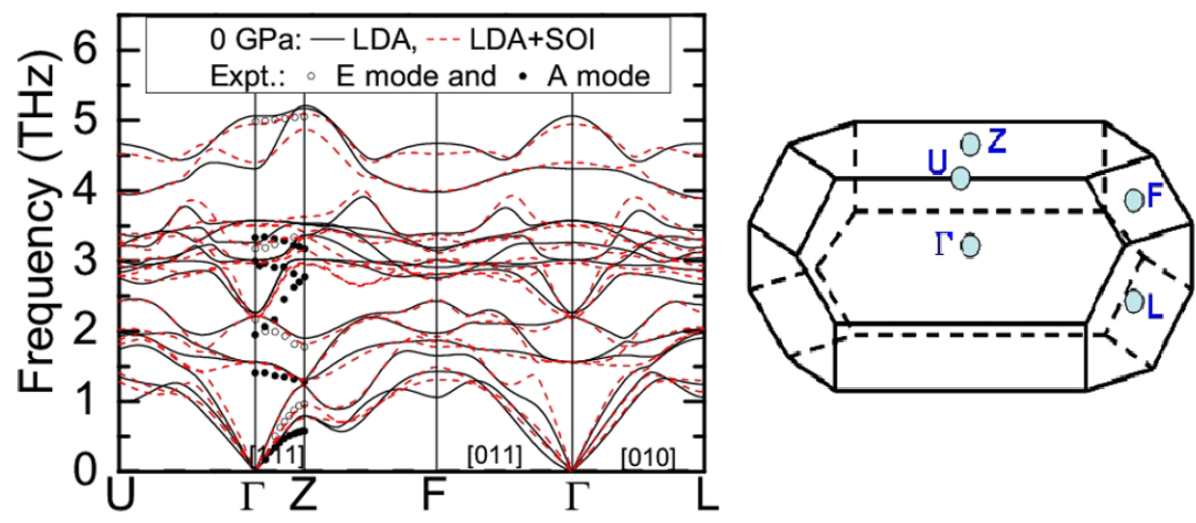

Figure 2.2: Phonon dispersion relations of $\mathrm{Sb}_{2} \mathrm{Te}_{3}$. Reproduced from B.-T. Wang, P. Souvatzis, O. Eriksson, and P. Zhang, J. Chem. Phys., 142:174702, 2015, with the permission of AIP Publishing. DOI: 10.1063/1.4919683 [80]

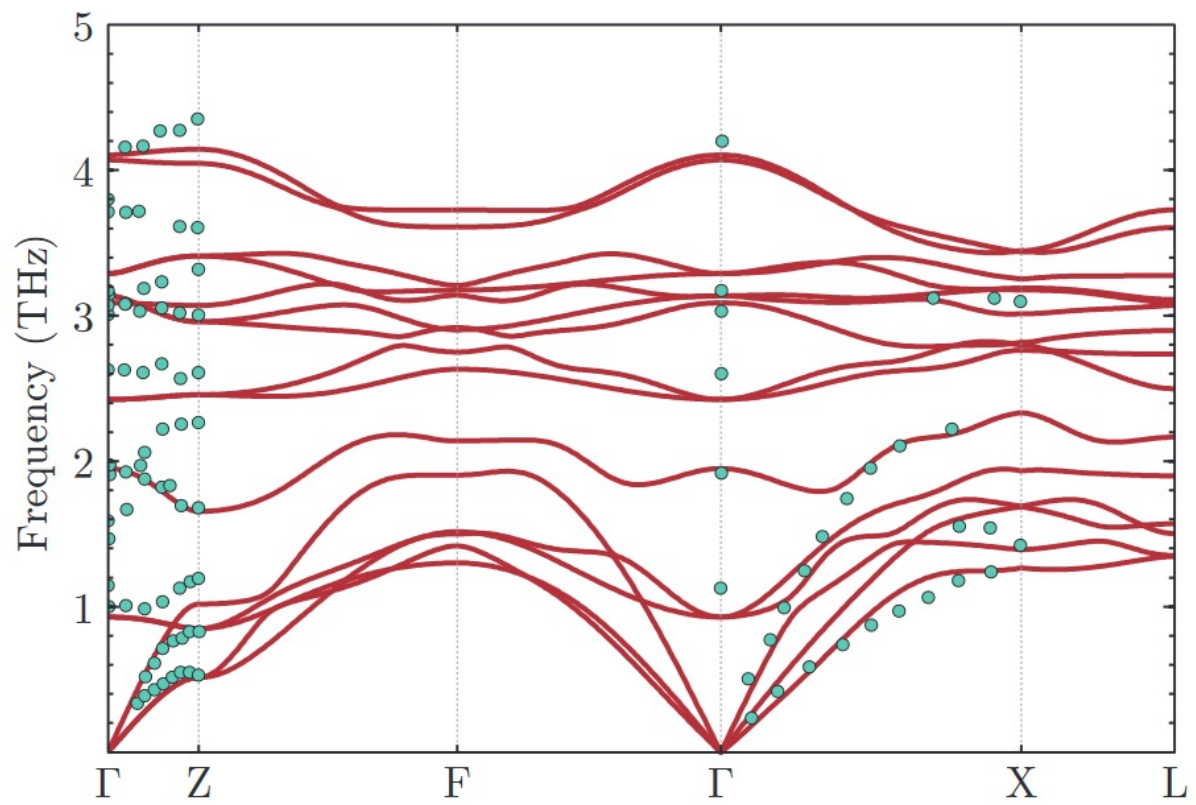

Figure 2.3: Phonon dispersion relations of $\mathrm{Bi}_{2} \mathrm{Te}_{3}$. Reprinted figure with permission from O. Hellman and D. A. Broido, Phys. Rev. B, 90:134309, 2014. Copyright 2019 by the American Physical Society. DOI: 10.1103/PhysRevB.90.134309 [82]

In this work, $\left(\mathrm{Sb}_{1-\mathrm{x}} \mathrm{Bi}_{\mathrm{x}}\right)_{2} \mathrm{Te}_{3}$ films deposited on (001) oriented Sapphire $\left(\alpha-\mathrm{Al}_{2} \mathrm{O}_{3}\right)$ substrates have been investigated. The space group of $\alpha-\mathrm{Al}_{2} \mathrm{O}_{3}$ is $R \overline{3} c$, No.167, and thus structurally related to the one of $\left(\mathrm{Sb}_{1-\mathrm{x}} \mathrm{Bi}_{\mathrm{x}}\right)_{2} \mathrm{Te}_{3}$ which is $R \overline{3} \mathrm{~m}$, No.166. However, the atomic binding forces in Sapphire with its predominant ionic bonds are significantly higher.

The thermal properties of $\alpha-\mathrm{Al}_{2} \mathrm{O}_{3}$ and $\mathrm{Sb}_{2} \mathrm{Te}_{3}$ respectively $\mathrm{Bi}_{2} \mathrm{Te}_{3}$ strongly deviate from each other. One reason for the different thermal properties can directly seen in very dif- 
ferent phonon dispersion relations:

The acoustic phonon modes in $\alpha-\mathrm{Al}_{2} \mathrm{O}_{3}$ [83] have much higher maximum frequencies than those of $\mathrm{Sb}_{2} \mathrm{Te}_{3}$ and they actually overtop the maximum frequencies of the optical modes of the latter, while in $\mathrm{Bi}_{2} \mathrm{Te}_{3}$ actually two optical modes lie within the acoustic frequency range. The optical phonon modes in $\alpha-\mathrm{Al}_{2} \mathrm{O}_{3}$ are much higher, although the ratio between highest optical and highest acoustic phonon frequencies is lower for $\alpha-\mathrm{Al}_{2} \mathrm{O}_{3}$ compared to $\mathrm{Sb}_{2} \mathrm{Te}_{3}$ and $\mathrm{Bi}_{2} \mathrm{Te}_{3}$.

The comparison shows: While in $\alpha-\mathrm{Al}_{2} \mathrm{O}_{3}$ acoustic phonons with high velocities are present over a huge frequency range, the covered frequency ranges of acoustic phonons in $\mathrm{Sb}_{2} \mathrm{Te}_{3}$ and $\mathrm{Bi}_{2} \mathrm{Te}_{3}$ are much lower.

\subsubsection{Thermal conductivity}

$\mathrm{Sb}_{2} \mathrm{Te}_{3}$ and $\mathrm{Bi}_{2} \mathrm{Te}_{3}$ have rather low and anisotropic thermal conductivities. This does have in principle two origins: Though they are semiconducting materials with quite high electrical conductivities, the latter are still some magnitudes lower than those of metals where the electrons play an important if not dominating role for the thermal conductivity.

The second origin is more specific and will be discovered in this subsection: The lattice thermal conductivity is very low, especially parallel to the c-axis $\left(\kappa_{c, l}\right)$. Two main reasons can be identified:

- A low sound velocity and thus phonon propagation velocity for acoustic modes Particularly parallel to the c-axis $\left(v_{c}\right)$. This comes from small bulk moduli $K$ and shear moduli $G$ due to the van der Waals bonded layers. As done in [84] the sound velocity of $\mathrm{Sb}_{2} \mathrm{Te}_{3}$ (parallel to the c-axis) can be calculated out of that moduli in form of elements of the stiffness matrix: $v_{c}=\frac{1}{3} v_{L}+\frac{2}{3} v_{T}=\frac{1}{3} \sqrt{\frac{C_{33}}{\rho}}+\frac{2}{3} \sqrt{\frac{C_{44}}{\rho}}=1383 \frac{\mathrm{m}}{\mathrm{s}}$

Due to a higher density and even lower bulk and shear moduli $v_{c}$ of $\mathrm{Bi}_{2} \mathrm{Te}_{3}$ is lower: $v_{c}=1057 \frac{\mathrm{m}}{\mathrm{s}}$.

For the calculation of both sound velocities, reference [85] has been used for the required elements of the stiffness matrix.

- A low intrinsic mean free path of phonon $l_{m f p}$ due to pronounced Umklapp scattering. One cause of this is high anharmonicity of the potential due to the van der Waals bonds. But also resonant bonding within the quintuple layers is an important source for high anharmonicity [79]. In the ternary system, $l_{m f p}$ is further reduced by Rayleigh scattering at $\mathrm{Sb}$ respectively Bi atoms. The influence of doping on both Rayleigh and Umklapp scattering has been investigated by the author of this thesis, see chapter 5 .

The group velocities of optical phonons are even lower than those of acoustic ones and since they are more sensitive to Rayleigh scattering, the contributions of optical phonons to thermal conductivity is limited and strongly depends on point defect densities. Due 
to the vdW layered structure, acoustic phonon frequencies and velocities of $\mathrm{Sb}_{2} \mathrm{Te}_{3}$ along the c-axis are even lower than those in the a,b-plane.

The pronounced Umklapp scattering is crucial for the low lattice thermal conductivity and the fundamental processes are still not fully understood. Therefore here the current state of research shall be outlined. A detailed discussion can be found in chapter 5 .

The highly anharmonic potentials of both $\mathrm{Sb}_{2} \mathrm{Te}_{3}$ and $\mathrm{Bi}_{2} \mathrm{Te}_{3}$ have been investigated by theoretical $[82,86,87]$ as well as by experimental studies $[53,88]$. For the Grüneisen parameter $\gamma_{G}=\frac{\alpha_{V}}{C_{V} K_{T}}$, often used as a measure of anharmonicity of a material, for $\mathrm{Sb}_{2} \mathrm{Te}_{3}$ and $\mathrm{Bi}_{2}$ Te values of 1.7 respectively 1.5 have been found [81]. Based on an increasing $\gamma_{G}$ by substitution of Te by Se atoms, $2.65 \pm 0.013$ for $\mathrm{Sb}_{2} \mathrm{Te}_{2} \mathrm{Se}$ [53], a comparable effect on the anharmonicity in the ternary system $\left(\mathrm{Sb}_{1-\mathrm{x}} \mathrm{Bi}_{\mathrm{x}}\right)_{2} \mathrm{Te}_{3}$ can be assumed.

However, the relatively low Grüneisen parameters cannot fully explain the oberserved low lattice thermal conductivities. In $\mathrm{Bi}_{2} \mathrm{Te}_{3}$ transverse optical (TO) modes with frequencies partially below the longitudinal acoustic (LA) mode, which further soften with increasing temperature, may lead to huge transition probabilities of Umklapp processes [82]. Furthermore, doping induced disorder is suspected to lead to amplified selected phonon scattering channels [89].

Strong Umklapp scattering requires high anharmonicity. Besides the bonding types of different strength, resonant bonding could be a reason for the huge anharmonicity in $\left(\mathrm{Sb}_{1-\mathrm{x}} \mathrm{Bi}_{\mathrm{x}}\right)_{2} \mathrm{Te}_{3}$. Long-ranged interactions in materials with resonant bondings are seen as an origin of the softening of low frequency TO modes leading to high anharmonicity [90].

\subsubsection{Kapitza resistances}

For the exact determination of the thermal conductivity of a material, one has to consider so called Kapitza resistances, also known as thermal boundary resistances or interfacial thermal resistances. The eponym was Peter Kapitza (Pyotr Kapitsa) who in 1941 reported a drop of temperature close to the interface of liquid helium II and a solid [91]. Already in 1936 Keesom and Keesom found the thermal resistance between copper and liquid helium II "relatively very considerable" [92], while another work from the same year neglected thermal boundary resistances [93]. Theoretical explanations including the important Acoustic Mismatch Modell (AMM) were found in the 1950s, a comprehensive review article has been written by Swartz and Pohl in 1989 [94].

The AMM explains Kapitza resistances at "perfectly" smooth interfaces by a mismatch of phonon modes in the joined materials. The so called Diffuse Mismatch Modell (DMM) deals with rough interfaces, taking diffuse scattering of phonons at the interface into account. 
In general, three parameters can be identified which lead to a high Kapitza resistance at the interface of two materials:

1. Different crystal structures and lattice parameters

2. Strongly deviating Debye temperatures

3. Different types of heat conduction: Electrons / Phonons

In case of a $\mathrm{Sb}_{2} \mathrm{Te}_{3}$ film on a $\alpha-\mathrm{Al}_{2} \mathrm{O}_{3}$ substrate, there is a considerable mismatch regarding lattice parameters. Additionally, van der Waals bonding leads to a high interfacial boundary resistance. The Debye temperatures are very different (165 [69] compared to $1042 \mathrm{~K}$ [95]). In combination this leads to the already discussed very different phonon dispersion relations and gives rise to a high acoustic mismatch. Additionally, in $\mathrm{Sb}_{2} \mathrm{Te}_{3}$ charge carriers significantly contribute to heat transport, while the electrically strongly insulating material $\alpha-\mathrm{Al}_{2} \mathrm{O}_{3}$ only exhibits lattice thermal conductivity.

Often the inverse of the Kapitza resistance, the so called thermal conductance $G$ is used expressing the heat flowing through an interface of defined area in the presence of a specific temperature gradient. High values have been obtained from timedomain thermoreflectance (TDTR) measurements for TiN films on different insulating substrates $\left(\mathrm{MgO}(001), \mathrm{MgO}(111)\right.$ and $\left.\mathrm{Al}_{2} \mathrm{O}_{3}(0001)\right)$ : Costescu et al. reported a value of $G \approx 7 \times 10^{8} \frac{\mathrm{W}}{\mathrm{m}^{2} \mathrm{~K}}[96]$. Even higher conductances (lower Kapitza resistances) have been measured at interfaces formed by the fcc metals $\mathrm{Al}$ and $\mathrm{Cu}$ which both show high electrical conductivities: A room temperature conductance of $G=4 \times 10^{9} \frac{\mathrm{W}}{\mathrm{m}^{2} \mathrm{~K}}$ has been reached [97]. The other extreme are interfaces of $\mathrm{Bi}$ and hydrogen-terminated diamond with a minimum room temperature value of $G=8 \times 10^{6} \frac{\mathrm{W}}{\mathrm{m}^{2} \mathrm{~K}}[98]$.

\subsubsection{State of actual research on $\mathrm{Sb}_{2} \mathrm{Te}_{3}$ and $\mathrm{Bi}_{2} \mathrm{Te}_{3}$ thin films}

Investigation of thin films has many advantages: On the one hand the required amount of material is small, on the other hand there are many established methods available for synthesizing films with very defined and reproducible properties. Additionally, the detailed investigation of such films can be performed by different well known techniques.

$\mathrm{Sb}_{2} \mathrm{Te}_{3}$ and $\mathrm{Bi}_{2} \mathrm{Te}_{3}$ thin films have been synthesized by many different methods during the last years. A selection of interesting publications is given below. In part, the motivation of the respective publication is included due to the fact that especially for $\mathrm{Sb}_{2} \mathrm{Te}_{3}$ thermoelectricity is only one of many promising applications.

\section{- Atomic Layer Deposition (ALD)}

Deposition of highly textured $\mathrm{Sb}_{2} \mathrm{Te}_{3}$ films on $\mathrm{Si} / \mathrm{SiO}_{2}$ substrates at $80^{\circ} \mathrm{C}$. This low deposition temperature allowed special lithography steps paving the way for elaborate measurement geometries. Room temperature measurements results: A Seebeck coefficient of $S=146 \frac{\mathrm{\mu V}}{\mathrm{K}}$, a mobility of $\mu=270.5 \times 10^{-4} \frac{\mathrm{m}^{2}}{\mathrm{Vs}}$, a low carrier 
concentration of $2.4 \times 10^{18} \frac{1}{\mathrm{~cm}^{3}}$ and therefore a low electrical conductivity of $\sigma=10^{4} \frac{\mathrm{S}}{\mathrm{m}}$. The low carrier concentration was interpreted as a result of low defect content and hence ALD was presented as advantageous compared to MBE where the films show ten times higher carrier concentrations.

Motivation: Precise measurement of thermoelectric relevant properties at $\mathrm{Sb}_{2} \mathrm{Te}_{3}$ films. (Research founded by the SPP1386 of the DFG that also founded parts of my work) [99]

Polycrystalline $\mathrm{Bi}_{2} \mathrm{Te}_{3}$ films have been deposited on native $\mathrm{SiO}_{2}$, soda-lime glass and covered silicon substrates with substrate temperatures between 160 and $350{ }^{\circ} \mathrm{C}$. The films have been investigated by XRD, XRR, EDX, SEM and TEM. Regular growth along the c-axis and decreasing growth rates with increasing temperature up to $250^{\circ} \mathrm{C}$, above no film growth was observed. With growing thickness/increasing number of cycles the roughness and the ratio of other orientations increase $((015)$ orientation already detectable for thicknesses below $20 \mathrm{~nm})$. In-plane electrical conductivities mainly between approximately $\sigma=1 \times 10^{5} \frac{\mathrm{S}}{\mathrm{m}}$ and $1 \times 10^{6} \frac{\mathrm{S}}{\mathrm{m}}$ were measured, but the authors itself question the reliability of the 4-point probe due to low film thicknesses. The obtained Seebeck coefficient for the film grown at $160^{\circ} \mathrm{C}$ was $S=-180 \frac{\mu \mathrm{V}}{\mathrm{K}} \cdot[100]$

\section{- Molecular Beam Epitaxy (MBE)}

Highly (001) textured (polycrystalline) $\mathrm{Sb}_{2} \mathrm{Te}_{3}$ films have been deposited on $\mathrm{Si}$ (111) substrates at $280^{\circ} \mathrm{C}$. Mobility measured at an $121 \mathrm{~nm}$ thick film was determined to be $\mu=305 \times 10^{-4} \frac{\mathrm{m}^{2}}{\overline{\mathrm{Vs}}}$ and an electrical conductivity of $\sigma=10.36 \times 10^{4} \frac{\mathrm{S}}{\mathrm{m}}$ has been measured. A decrease of electrical conductivity with decreasing film thickness has been observed, for a $28 \mathrm{~nm}$ thick film $\sigma=4.257 \times 10^{4} \frac{\mathrm{S}}{\mathrm{m}}$ has been measured. The mean free path of charge carriers has been found to be dependent on grain sizes and defects.

Motivation: Basic research on material parameters of $\mathrm{Sb}_{2} \mathrm{Te}_{3}$ with regard to thermoelectric application, usage of the crystalline-amorphous phase change, and usage of topological insulators. [101]

Thin films of $\mathrm{Bi}_{2} \mathrm{Te}_{3}$ have been grown on $\mathrm{Si}(111)$ substrates with widely varied deposition parameters. Substrate temperature $T_{S}$ was varied in the range from 200 to $600{ }^{\circ} \mathrm{C}$. For $\mathrm{T}_{\mathrm{S}}=350{ }^{\circ} \mathrm{C}, \mathrm{T}_{\mathrm{S}}=400^{\circ} \mathrm{C}$ and $\mathrm{T}_{\mathrm{S}}=450{ }^{\circ} \mathrm{C}(001)$ oriented growth has been shown by XRD. It has been found that during the cooling, until $\mathrm{T}_{\mathrm{S}} \leq 350^{\circ} \mathrm{C}$ a Te atmosphere has to been maintained in order to obtain films of good quality. Furthermore Te out-diffusion and desorption processes strongly have to be considered. Angular-resolved photoelectron spectroscopy (ARPES) has been performed revealing a Dirac cone which is specific for topological insulators. [102] 


\section{- Ion Beam Sputtering (IBS)}

$\mathrm{Sb}_{2} \mathrm{Te}_{3}$ films have been prepared at room temperature on BK7 glass (a borosilicate glass). XRD shows „poor crystalline quality“ of the polycrystalline films, after annealing at $400{ }^{\circ} \mathrm{C}$ for $1 \mathrm{~h}$, a $(205)$ orientation is dominating. The power factor was increased from $0.4 \times 10^{-3} \frac{\mathrm{W}}{\mathrm{mK}^{2}}$ to $2.26 \times 10^{-3} \frac{\mathrm{W}}{\mathrm{mK}^{2}}$ by annealing. Seebeck coefficient after annealing was $S=106 \frac{\mu \mathrm{V}}{\mathrm{K}}$ and an electrical conductivity of $\sigma=20.1 \times 10^{4} \frac{\mathrm{S}}{\mathrm{m}}$ has been measured.

Motivation: Method for preparing $\mathrm{Sb}_{2} \mathrm{Te}_{3}$ films with good thermoelectric properties. [103]

Ion beam sputtered polycrystalline $\mathrm{Bi}_{2} \mathrm{Te}_{3}$ films on glass substrates have been investigated. Substrate temperatures $T_{S}$ have been varied between 100 and $350{ }^{\circ} \mathrm{C}$. For substrate temperatures above $250^{\circ} \mathrm{C}$ XRD indicated the c-axis to be the preferred crystallographic orientation. SEM shows an increasing grain size with increasing substrate temperatures, above $300^{\circ} \mathrm{C}$ an average grain size larger than $500 \mathrm{~nm}$ has been obtained. A maximum electric conductivity of $\sigma\left(T_{S}=300^{\circ} \mathrm{C}\right)=3.94 \times 10^{5} \frac{\mathrm{S}}{\mathrm{m}}$ and an optimum Seebeck coefficient of $S\left(T_{S}=250{ }^{\circ} \mathrm{C}\right)=-146 \frac{\mathrm{\mu V}}{\mathrm{K}}$ have been reported (direction of measurement is not explicitely mentioned). The highest power factor has been obtained at a substrate temperature of $T_{S}=300^{\circ} \mathrm{C}: 6.45 \times 10^{-3} \frac{\mathrm{W}}{\mathrm{mK}^{2}}$. [104] This publication illustrates the crucial role of optimized deposition parameters in order to get usable thermoelectric films. It is necessary to comment on the very high $\sigma$ leading to the high power factor. It is a very high value and should be regarded critically. However, even if true it would lead to a high electronic contribution to $\kappa$ and so it would have a negative impact on $Z T$.

\section{- Radio Frequency Magnetron Sputtering (RFMS)}

Deposition of $\mathrm{Sb}_{2} \mathrm{Te}_{3}$ films (amongst other materials) on $\mathrm{Si}$ (100), $\mathrm{Si}$ (111) and amorphous $\mathrm{SiO}_{2}\left(\mathrm{a}-\mathrm{SiO}_{2}\right)$ at deposition temperatures between 200 and $250{ }^{\circ} \mathrm{C}$. For the $\mathrm{Si}$ substrates formation of an amorphous $\mathrm{Si}(\mathrm{a}-\mathrm{Si}$ ) layer has been observed due to Ar ion bombardment. Still (001) growth of the $\mathrm{Sb}_{2} \mathrm{Te}_{3}$ films. Growth on a- $\mathrm{SiO}_{2}$ led to poorly oriented films, explained by the reaction of $\mathrm{O}$ atoms with $\mathrm{Te}$ inhibiting the formation of quintuple layers. A previous sputtering of $50 \mathrm{~nm}$ a-Si on the substrate led to highly (001) oriented films. Generally before the of quintuple layer formation, a termination with a monoatomic Te layer is assumed.

Motivation: The use of topological insulators in spintronics and quantum computing. [105]

Polycrystalline $\mathrm{Bi}_{2} \mathrm{Te}_{3}$ thin films have been synthesized by $\mathrm{RF}$ magnetron cosputtering on $\mathrm{SiO}_{2} / \mathrm{Si}$ substrates without intentional heating. Subsequently the effect of different annealing temperatures between 150 and $350{ }^{\circ} \mathrm{C}$ has been studied. For all samples, different crystallographic orientations are visible in the XRD 
patterns, while the as-deposited sample has the broadest peaks. Higher annealing temperatures turned out to increase crystallinity enhancing the mobility of charge carriers. After annealing at $250^{\circ} \mathrm{C}$ a charge carrier concentration of $6.85 \times 10^{19} \frac{1}{\mathrm{~m}^{3}}$ has been determined. Using an annealing temperature of $300{ }^{\circ} \mathrm{C}$ an optimum Seebeck coefficient $S=-242 \frac{\mathrm{\mu V}}{\mathrm{K}}$ and a maximum power factor of $2.1 \times 10^{-3} \frac{\mathrm{W}}{\mathrm{mK}^{2}}$ have been found. [106]

Out of these data one can calculate an electric conductivity of $\sigma=3.6 \times 10^{4} \frac{\mathrm{S}}{\mathrm{m}}$ at $300{ }^{\circ} \mathrm{C}$ which is quite reasonable and comparable to other literature data. However, one has to note that the given values for $\sigma$ in this publication are contradictory and too high concerning the magnitude. Nevertheless, this publication is worth mentioning since it demonstrates the significant correlation between annealing temperature and thermoelectric performance.

\section{- Pulsed Laser Deposition (PLD)}

Growth of (001) textured polycrystalline $\mathrm{Sb}_{2} \mathrm{Te}_{3}$ on $\mathrm{Si}$ (111) substrates. Different deposition temperatures between room temperature and $280^{\circ} \mathrm{C}$ have been used. For deposition temperatures between 170 and $240^{\circ} \mathrm{C}$, XRD shows intense and narrow peaks indicating highly textured films of good crystalline quality. HRTEM shows regularly grown quintuple layers for the film deposited at $240^{\circ} \mathrm{C}$.

Motivation: Basic research on thin film synthesis of Phase Change Materials (PCMs), which often use GeTe and $\mathrm{Sb}_{2} \mathrm{Te}_{3}$ as starting materials. [107]

Varying different deposition parameters, a Nd:YAG laser has been used to grow $\mathrm{Bi}_{2} \mathrm{Te}_{3}$ thin films. Substrate temperature was kept at $295 \mathrm{~K}$ for every sample. The films have been analyzed by SEM, XRD, microprobe and SIMS. Since the desired stoichiometry could be reached, the authors claim an advantage towards other methods concerning room temperature deposition. No preferred growth direction could be identified by XRD. [108]

\section{- Thermal (co-)evaporation}

Polycrystalline $\mathrm{n}-\mathrm{Sb}_{2} \mathrm{Te}_{3}$ films (Te rich) have been deposited on polyimide foil (as substrate) by thermal evaporation using substrate temperatures between 150 and $220^{\circ} \mathrm{C}$. For a film deposited at $220^{\circ} \mathrm{C}$, parallel to the substrate a thermal conductivity of $\kappa=1.8 \frac{\mathrm{W}}{\mathrm{mK}}$ and a Seebeck coefficient of $S=160 \frac{\mathrm{\mu V}}{\mathrm{K}}$ were measured and a $Z T$ value of 0.3 has been determined. [109]

Almost single crystalline (001) oriented $\mathrm{Sb}_{2} \mathrm{Te}_{3}$ films have been grown on (001) oriented $\alpha-\mathrm{Al}_{2} \mathrm{O}_{3}$ substrates by Bendt et al. (partners of the author of this thesis within the joint DFG project) in a cold-wall reactor and temperature series have been conducted for substrate $\left(300-400^{\circ} \mathrm{C}\right)$ respectively evaporator $\left(540-620^{\circ} \mathrm{C}\right)$. The samples have been investigated via XRD, XPS, HRTEM (by the author of this thesis) and electric transport measurements. Films of best quality have 
been obtained for an evaporator temperature of $580{ }^{\circ} \mathrm{C}$ combined with a substrate temperature of $350^{\circ} \mathrm{C}$. For such a film, at $300 \mathrm{~K}$ an in-plane electrical conductivity of $3.95 \times 10^{5} \frac{\mathrm{S}}{\mathrm{m}}$ and a high carrier mobility of $558 \frac{\mathrm{cm}^{2}}{\mathrm{Vs}}$ have been measured. [110] see Appendix 2 of this thesis

At such (001) oriented $\mathrm{Sb}_{2} \mathrm{Te}_{3}$ films of different thickness, out-of-plane thermal conductivities have been measured by the author of this thesis. The measurements revealed a low intrinsic $\kappa_{c}$. Detailed HRTEM and STEM-HAADF investigations have been done, including an EELS analysis of the substrate-film interface. [84] (see chapter 4 of this thesis)

Polycrystalline $\mathrm{n}-\mathrm{Bi}_{2} \mathrm{Te}_{3}$ films have been deposited on $25 \mu \mathrm{m}$ thick polyimide foil (due to the low thermal conductivity of this material and its thermal expansion coefficient close to that of the telluride film). The influence of $\mathrm{Bi}$ and Te evaporation rates and substrate temperatures has been studied in detail and more than 100 samples have been prepared and investigated by a variety of methods. An optimum Seebeck coefficient of $S=-250 \frac{\mathrm{\mu V}}{\mathrm{K}}$ could be achieved. The highest power factor of $4.9 \times 10^{-3} \frac{\mathrm{W}}{\mathrm{mK}^{2}}$ has been obtained at a substrate temperature of approximately $270^{\circ} \mathrm{C}$. By combination of their measurement results with literature data, the authors claim a $Z T$ value close to unity. [111]

\section{- Metal Organic Chemical Vapour Deposition (MOCVD)}

(001) oriented $\mathrm{Sb}_{2} \mathrm{Te}_{3}$ films have been deposited on $\alpha-\mathrm{Al}_{2} \mathrm{O}_{3}$ substrates at $400{ }^{\circ} \mathrm{C}$ by Bendt et al. (partners of the author of this thesis within the joint DFG project). Deposition rates of $3 \frac{\mathrm{\mu m}}{\mathrm{h}}$ have been reached. The measured sheet resistivity increased by more than a factor 10 between 2 and $400 \mathrm{~K}$, a carrier concentration above $10^{19} \frac{1}{\mathrm{~cm}^{3}}$ has been measured. The latter is explained by defects, most likely antisite defects which occur frequently in $\mathrm{Sb}_{2} \mathrm{Te}_{3}$. [112]

A modified MOCVD method has been used for synthesizing $\mathrm{Bi}_{2} \mathrm{Te}_{3}$ films: The metal organic sources were mixed with hydrogen gas in a special mixing room. This resulted in a significantly increased growth rate with a maximum value of $7 \frac{\mathrm{\mu m}}{\mathrm{h}}$ at a mixing room temperature of $300^{\circ} \mathrm{C}$ while the substrate temperature was fixed at $400^{\circ} \mathrm{C}$ for all samples. However, the authors state that high mixing room temperatures have a negative impact on thermoelectric properties. The optimum Seebeck coefficient obtained was $-225 \frac{\mathrm{\mu V}}{\mathrm{K}}$ and the highest power factor was $1.86 \times 10^{-3} \frac{\mathrm{W}}{\mathrm{m} \mathrm{K}^{2}}$. [113] 


\subsubsection{Superlattices and solid solutions}

The beneficial thermoelectric properties of the solid solution $\left(\mathrm{Sb}_{1-\mathrm{x}} \mathrm{Bi}_{\mathrm{x}}\right)_{2} \mathrm{Te}_{3}$ in comparison to the binary compounds and a minimum lattice thermal conductivity $\kappa_{1}$ at 20 to 40 at.\% $\mathrm{Bi}_{2} \mathrm{Te}_{3}$ have been identified already 6 decades ago [5]. Subsequent research dealt with a variety of different manufacturing methods and also additional alloying elements. Substitution of Te by Se is just one of many examples.

Since $\mathrm{Sb}_{2} \mathrm{Te}_{3}$ and $\mathrm{Bi}_{2} \mathrm{Te}_{3}$ are known as topological insulators, recent works mainly use a nanostructuring approach. One idea is to increase phonon scattering and simultaneously increase electric conductivity via topologically protected states by increasing the total surface of grain boundaries. Another important aspect is the development of scalable methods in order to come closer to economically justifiable synthesis procedures.

For nanoplatelets with $\mathrm{Bi}$ concentration $x=0.25$ prepared by solvothermal synthesis, a $Z T$ of $\sim 0.51$ at $375 \mathrm{~K}$ has been reported. The authors interpret the result as promising towards practical thermoelectric applications. They further state that the platelets can be sintered into bulk pellets. [114]

Serrano-Sánchez et al. used an arc-melting procedure to obtain solid solution samples with different Bi contents. For a sample with measured composition $\mathrm{Sb}_{1.49(1)} \mathrm{Bi}_{0.51(1)} \mathrm{Te}_{3}$ a high Seebeck coefficient of $S=350 \frac{\mathrm{\mu V}}{\mathrm{K}}$ and a high figure of merit $Z T=1.1$ have been measured at $T=395 \mathrm{~K}$. [115]

Serrano-Sánchez et al. claim increased phonon scattering at grain boundaries which seems reasonable since the published $\kappa(T)$ data for the $\mathrm{Sb}_{2} \mathrm{Te}_{3}$ sample shows a monotonous increase between 5 and $320 \mathrm{~K}$. Such a behavior is only explainable by heat transport via charge carriers being the dominant thermal conductivity mechanism.

In $2015 \mathrm{Kim}$ et al. stated to have reached a $Z T$ of 1.86 at $320 \mathrm{~K}$ for $\left(\mathrm{Sb}_{0.75} \mathrm{Bi}_{0.25}\right)_{2} \mathrm{Te}_{3}$ bulk alloys. The authors justify this high value by lowering the lattice thermal conductivity via the scattering of midfrequency phonons at dense dislocation arrays. [23] In this context, recent research on $\left(\mathrm{Sb}_{1-\mathrm{x}} \mathrm{Bi}_{\mathrm{x}}\right)_{2} \mathrm{Te}_{3}$ solid solution samples is promising. However, separating of intrinsic and microstructural effects is not possible with such samples. This is in particular the case since the material exhibits complex temperature dependent structure-property correlations. To evaluate the impact of different nanostructuring methods, comprehensive and systematical studies of well defined solid solution samples are the essential basis.

Another approach is the creation of thin film superlattices to further decrease the thermal lattice conductivity by utilization of acoustic mismatches at interfaces. A sensational publication regarding this topic was published in Nature by Venkatasubramian et al. in 2001 [7]. It gave rise to new interest and efforts in global research on thermoelectric materials, especially concerning thin films and nano structured materials. The authors reported a $Z T$ value at $300 \mathrm{~K}$ of 2.4 for p-type $\mathrm{Bi}_{2} \mathrm{Te}_{3}-\mathrm{Sb}_{2} \mathrm{Te}_{3}$ superlattices with single layer thicknesses of $d\left(\mathrm{Bi}_{2} \mathrm{Te}_{3}\right) / d\left(\mathrm{Sb}_{2} \mathrm{Te}_{3}\right)=0.2 \mathrm{~nm} / 0.3 \mathrm{~nm}$ grown by MOCVD. Lattice thermal conductivity values of superlattices with different thicknesses have already been published in 2000 and reach a very low value of $\kappa_{l}=0.22 \frac{\mathrm{W}}{\mathrm{mK}}$ [116] for the high ZT superlattices. 
However, to date the outstanding results of ref. [7] could not be reproduced by any other group. A detailed review report by Winkler et al. [8] deals with superlattices deposited by different methods finding that a significant reduction of $\kappa_{1}$ could not be achieved.

Furthermore, for $\mathrm{Bi}_{2} \mathrm{Te}_{3} /\left(\mathrm{Sb}_{0.1} \mathrm{Bi}_{0.9}\right)_{2} \mathrm{Te}_{3}$ superlattices with single layer thicknesses $25 \mathrm{~nm} / 25 \mathrm{~nm}$ grown by a sputtering process, a strong increase of roughness and the formation of grains have been observed after an annealing process at only $150{ }^{\circ} \mathrm{C}$ for 2 h. [78]

MBE grown $\mathrm{Bi}_{2} \mathrm{Te}_{3} / \mathrm{Sb}_{2} \mathrm{Te}_{3}$ superlattices with single layer thicknesses of $1 \mathrm{~nm} / 5 \mathrm{~nm}$ have been shown by XRD and TEM to not being stable at elevated temperatures due to interdiffusion processes. Interdiffusion has been noticed already at $200^{\circ} \mathrm{C}$. [117]

A review article published in 2016 [9] again comes to the conclusion that the results from 2001 could not be reproduced. The authors argue that compensation effects between different majority charge carriers in the superlattices might lead to the fact that the high Seebeck coefficient, high carrier mobility and subsequently the high power factor reported by ref. [7] could not be reproduced.

Since the MOCVD superlattices with the high $Z T$ value [7] and low lattice thermal conductivity [116] have been grown at temperatures of approximately $225^{\circ} \mathrm{C}$ [118], it cannot be excluded that interdiffusion already took place during the growth process. The TEM images shown in ref. [118] are lacking of quality in order to judge whether interdiffusion has taken place respectively to which extend. However, some kind of irregularities can be identified there. In any case, the application temperature windows of such superlattices would be very limited foiling many application ideas concerning TEGs. With regard to technical applications one also has to consider the elaborate production process compared to single layer films.

Conclusive it can be stated, that superlattices of $\mathrm{Sb}_{2} \mathrm{Te}_{3}$ and $\mathrm{Bi}_{2} \mathrm{Te}_{3}$ are rather impractical for technical applications and further research does not seem to be worthwhile. However, $\left(\mathrm{Sb}_{1-\mathrm{x}} \mathrm{Bi}_{\mathrm{x}}\right)_{2} \mathrm{Te}_{3}$ thin films and bulk materials show promising thermoelectric properties and the synthesis possibilities are diverse. Microstructure, doping levels and related properties are well tunable. For research purposes growing methods are usually implemented on small scales, but many of them are scalable and some are already established in industrial processes for a long time. 



\section{CHAPTER 3}

\section{Experimental techniques}

For the purpose of establishing structure property relations between thermal conductivity and the defects which mainly dominate the phonon mean free path, main experimental methods have been the $3 \omega$ technique for measuring cross-plane thermal conductivities of the $\left(\mathrm{Sb}_{1-\mathrm{x}} \mathrm{Bi}_{\mathrm{x}}\right)_{2} \mathrm{Te}_{3}$ thin films and High Resolution Transmission Microscopy (HRTEM) for investigating the microstructure of the films down to atomic resolution. For the $3 \omega$ measurements, conducted at a self-built setup, an insulation layer synthesis routine via sputtering has been developed by the author, including subsequent sputtering of $\mathrm{Pt}$ for the heating structures. This chapter outlines the most important experimental techniques which have been used, with an emphasis on $3 \omega$ setup and analysis.

\subsection{Sputtering}

Sputtering is a deposition process which belongs to the group of Physical Vapour Deposition (PVD) techniques. In sputtering processes, a plasma source creates ions which are subsequently accelerated by high voltages whereupon they eject atoms from a target made out of a specific material. This target may consist of one or more elements and the ejected atoms move to a substrate placed on a holder at a specific position, which may be heated up to a defined temperature. The incoming atoms form a continuously growing film on the substrate. Typical ion energies for sputtering processes are commonly in the range of 100 to $1000 \mathrm{eV}$ or beyond und thus much higher than the thermal energies. The ejected atoms also have energies in that range and therefore, the synthesized films exhibit a significant density of defects due to knock-on damage, compared to other methods which are more close to the thermodynamic equilibrium. On the other side this enables to influence the material parameters of sputtered films to a great extend. Sputtering processes need high vacuum, which is commonly created by a backing pump and a turbo molecular pump (TMP).

Commonly, AC and RF sources are used. For the reults presented in this thesis, an Ion Beam Sputtering (IBS) unit equipped with a RF source has been utilized. A RF plasma source works by creating electric (and magnetic) fields with an oscillation frequency of $13.56 \mathrm{MHz}$ (a special frequency which is chosen in order to not disturb radio communication by the resulting electromagnetic waves). Caused by the oscillating fields, already 
existing ions perform movements and thereby ionize atoms creating further ions. The operating principle of IBS is explained below, a TEM image showing a cross section of ion beam sputtered insulation layer and Pt heating structure on top of a $\mathrm{Sb}_{2} \mathrm{Te}_{3}$ film is shown in chapter 4 (Fig. S2).

\subsubsection{Ion Beam Sputtering}

In Ion Beam Sputtering, ions created by the ion source in a so called discharge chamber (the Forward power is supplied here) are accelerated by an accelerating grid (by applying the so called beam voltage). In case of the present work, Xe has been used as sputtering gas, therefore $\mathrm{Xe}^{+}$ions have been accelerated. The higher atomic mass of Xe compared to the often used Ar results in a higher sputtering rate.

Close to the accelerating grid a second grid is placed. At the second grid, a potential with reversed sign of the potential at the accelerating grid, is applied. It is repulsive for the ions and influences the shape of the ion beam. The applied voltage is called accelerator voltage.

In the neutralizer an electron emitting filament is located. The emitted electrons are accelerated by a relatively small voltage and recombine with the ion beam. The purpose of a neutralizer is to avoid charging effects at the target, the used Ar gas is needed for the operation of the neutralizer. The target itself is placed under a specific angle of commonly $45^{\circ}$ to the beam direction. The former $\mathrm{Xe}^{+}$ions hit the target as $\mathrm{Xe}$ atoms and eject target atoms. The angular distribution under which the target atoms leave the target depends on the specific atomic mass. While using targets consisting of only one element, the mass dependent angles do not play a significant role.

For the $3 \omega$ method an amorphous a-SiO $\mathrm{S}_{2}$ insulation layer was deposited via reactive IBS using $\mathrm{O}_{2}$ and subsequently, in the same sputter run, Pt was deposited for the $3 \omega$ heating structure. As Si and Pt atoms have different masses, I determined a compromise angle, where both compounds showed decent sputtering rates. Under this angle a substrate was placed on a holder. Commonly substrates in sputtering processes are heated up to a temperature that is beneficial for the growth of the desired compound. In my case due to an e-beam resist on top of the sample (necessary for achieving the heater geometry by a subsequent lift-off process) heating was not possible. However, the developed routine enabled depositing insulation layers without intentional heating.

The used parameters for IBS of $\mathrm{SiO}_{2}$ respectively Pt are shown in table 3.1. Note that the pressure values are quite rough due to calibration of the used Penning gauge to $\mathrm{N}_{2}$. To establish reproducible gas conditions, specific gas flows were adjusted by using a flow controller. The used gas flows have been: $2.3 \mathrm{sccm}$ for Xe, $3.6 \mathrm{sccm}$ for Ar and a fixed value was set at a manual needle valve for $\mathrm{O}_{2}$. 


\begin{tabular}{|l|c|c|}
\hline Parameter & $\mathrm{a}-\mathrm{SiO}_{2}$ & $\mathrm{Pt}$ \\
\hline Used target & $\mathrm{Si}$ & $\mathrm{Pt}$ \\
Substrate temperature $T\left[{ }^{\circ} \mathrm{C}\right]$ & $<100$ (not heated) & $<100$ (not heated) \\
Background pressure $p[\mathrm{mbar}]$ & $<3 \times 10^{-7}$ & $<3 \times 10^{-7}$ \\
Xe partial pressure $p_{\mathrm{Xe}}[\mathrm{mbar}]$ & $2.3 \times 10^{-4}$ & $2.3 \times 10^{-4}$ \\
Ar partial pressure $p_{\mathrm{Ar}}[\mathrm{mbar}]$ & $1.2 \times 10^{-4}$ & $1.2 \times 10^{-4}$ \\
O partial pressure $p_{\mathrm{O}_{2}}[\mathrm{mbar}]$ & $1.1 \times 10^{-3}$ & $<3 \times 10^{-7}$ \\
Forward power $P[\mathrm{~W}]$ & 53 & 50 \\
Beam voltage $U_{\mathrm{i}}[\mathrm{V}]$ & 1000 & 1000 \\
Beam current $I_{\mathrm{i}}[\mathrm{mA}]$ & 20 & 20 \\
Neutralizer voltage $U_{\mathrm{e}}[\mathrm{V}]$ & 20 & 20 \\
Neutralizer current $I_{\mathrm{e}}[\mathrm{mA}]$ & 25 & 25 \\
Accelerator voltage $U_{\mathrm{a}}[\mathrm{V}]$ & 100 & 200 \\
Deposition rate $R\left[\frac{\mathrm{nm}}{\mathrm{min}}\right]$ & 23 & \\
\hline
\end{tabular}

Table 3.1: Parameters used for IBS of a- $\mathrm{SiO}_{2}$ insulation layers and Pt heating structures. 


\subsection{The $3 \omega$ method}

\subsubsection{Fundamentals}

The $3 \omega$ method is a powerful technique for measuring thermal conductivities of bulk materials and in particular those of thin films. A small $3 \omega$ voltage occurring in a periodically with angular frequency $\omega$ heated wire has already been discovered in 1911 by Corbino [119]. Its utilization for measuring thermal conductivities of bulk materials was introduced by David G. Cahill in 1990 [120] and an extension for thin films was presented in 1994 by Cahill and coworkers [121]. The advantage of this method regarding thin films consists in a precise measurement of the cross-plane thermal conductivity at well defined temperatures. Concerning the latter, to the best knowledge of the author, no other method can compete.

The $3 \omega$ method is an indirect measurement: Electrical quantities and temperatures are measured, thermal conductivities are derived at the end. A heating structure (length $l$, width $2 b$ ) placed on top of the thin film (thickness $t_{\mathrm{f}}$ ) is electrically heated by a defined AC power $P$ at different angular frequencies $\omega$. The temperature fluctuation $\Delta T$ of the heater (Pt heating structure) is detected by measuring the following quantities:

- $U_{\omega}$ : The amplitude of the voltage that is applied between the ends of the heater.

- $U_{3 \omega}$ : The amplitude of a voltage with the triple frequency (third harmonic) of the original heating current. It results from the periodically fluctuating resistance of the heater.

- $R_{0}$ : The average resistance of the heater at a specific temperature.

- $d R / d T$ : The temperature gradient of the resistance of the heater.

The derivation of the connection between these quantities is:

$$
\begin{gathered}
I(t)=I_{0} \sin (\omega t) \\
P(t)=U(t) I(t)=R I^{2}(t)=\frac{R I_{0}^{2}}{2}(1-\cos (2 \omega t)) \\
R(t)=R_{0}(1+\alpha \Delta T(t))=R_{0}(1+\alpha \Delta T \cos (2 \omega t+\phi)) \quad, \quad \alpha=\frac{1}{R_{0}} \frac{d R}{d T} \\
U(t)=R(t) I(t)=R_{0} I_{0} \sin (\omega t)+\frac{R_{0} I_{0} \alpha \Delta T}{2}(\sin (3 \omega t+\phi)-\sin (\omega t+\phi)) \\
\Delta T=\frac{2 U_{3 \omega}}{U_{\omega} \alpha}=\frac{d T}{d R} \cdot \frac{2 R_{0} U_{3 \omega}}{U_{\omega}}
\end{gathered}
$$


Equation (3.1) shows the original heating current with angular frequency $\omega$. It leads to a heating power with double the frequency, equation (3.2). By Joule heating this applied power leads to a temperature fluctuation of the heater with the same angular frequency $2 \omega$, but shifted in time by a phase shift $\phi$, which results in a periodical fluctuation of the resistance, see equation (3.3). Multiplication with equation (3.1) reveals the resulting voltage between the ends of the heater, shown in equation (3.4). The important component of the total voltage is the $U_{3 \omega}$ voltage. Equation (3.5) finally shows the amplitude of the thermal oscillation of the heater $\Delta T$ expressed by the measured quantities.

$\Delta T$ subsequently is brought into connection with the thermal conductivities of film and substrate via a solution of the heat equation using specific boundary conditions. This is shown explicitly in the next subsections.

\subsubsection{The solution of the heat equation}

The evaluation of the $3 \omega$ measurement results needs a solution of the heat equation for the substrate under the heater. The thin film is then added as simple thermal offset, which is reasonable within specific boundary conditions shown later.

The general heat equation with a heat source $f(\vec{x}, t)$ is shown in equation (3.6). $\kappa, \rho$ and $C$ are the thermal conductivity, the density and the specific heat capacity of the material, $D$ is called thermal diffusivity and can be considered as a kind of thermal diffusion coefficient.

$$
\frac{\partial}{\partial t} T(\vec{x}, t)-D \nabla^{2} T(\vec{x}, t)=f(\vec{x}, t) \quad, \quad D=\frac{\kappa}{\rho C}
$$

Solutions of equation (3.6) for different cases can be found in ref. [122].

For a heater of infinite length, a 2 dimensional stationary solution $\left(\mathrm{r}=x^{2}+y^{2}\right)$ of the heat equation is derived by ref. [122] ( $x$ is parallel to the surface and perpendicular to the heater, $y$ is perpendicular to surface and heater). This solution shown in equation (3.7) is the starting point of the mathematical derivations for the $3 \omega$ method in ref. [120]:

$$
\begin{gathered}
\Delta T(r)=\frac{P}{l \pi \kappa_{s}} K_{0}(q r) \\
\frac{1}{q}=\sqrt{\frac{D}{i 2 \omega}}=\sqrt{\frac{\kappa_{s}}{i 2 \omega \rho C}}
\end{gathered}
$$

$P$ is the amplitude of the power applied at the heater, $l$ is the length of the heater, $\omega$ is the angular frequency of the heating current (see equation (3.1)), $\left|\frac{1}{q}\right|$ is the so called thermal penetration depth. $\kappa_{s}, \rho$ and $C$ are material parameters of the substrate. The boundary condition "infinite long heater" is well fulfilled in case of $l \gg 2 b$ and $l \gg\left|\frac{1}{q}\right|$. For that reason these two conditions always have to be kept in mind, otherwise the $3 \omega$ evaluation may lead to inaccurate results. 
$K_{0}$ is the zeroth-order modified Bessel function. For $r \ll\left|\frac{1}{q}\right|$ equation (3.7) can be further simplified to equation (3.9).

$$
\Delta T(r)=\frac{P}{l \pi \kappa_{s}}\left(\frac{1}{2} \ln \frac{D}{r^{2}}+\ln 2-0.5772-\frac{1}{2} \ln (2 \omega)-\frac{i \pi}{4}\right)
$$

The next step is to bring the heater width $2 b$ into play switching from a point source in $2 \mathrm{D}$ to a heater of finite width. This is done by Cahill [120] for the area close to the surface $(y=0)$ by means of a Fourier transformation and convolution. Thereby the real space coordinate $x$ is replaced by the Fourier space coordinate $k$. The average $\Delta T$ over the whole width $2 b$ of the heater is then given by equation (3.10). Now $\kappa_{s, y}$ is taken due to considering a domain close to the heater where the heat flow through the material goes approximately only in $\mathrm{y}$-direction (however, this is purely a question of designation).

$$
\Delta T=\frac{P}{l \pi \kappa_{s, y}} \int_{0}^{\infty} \frac{\sin ^{2}(k b)}{(k b)^{2} \sqrt{k^{2}+q^{2}}} d k
$$

For a small heater width $\left(b \ll\left|\frac{1}{q}\right|\right)$ equation (3.10) can be simplified to:

$$
\Delta T=-\frac{P}{2 l \pi \kappa_{s, y}}\left(\ln (\omega)+\frac{1}{2} \ln \frac{i b^{2}}{D}\right)+\text { const. }
$$

Using equation (3.11) the determination of $\kappa_{s, y}$ can easily be done by a linear fit of the real part of $\Delta T$ determined by equation (3.5) vs. $\ln \omega$. However, therefor the phase shift between the original heating current and the resulting $\Delta T$ oscillation has to be determined very precisely, which is very challenging and error-prone. Due to relatively small phase shifts it is even more difficult to get $\kappa_{s, y}$ using the imaginary part of $\Delta T$, so this approach is not an useful option.

For that reasons in the course of the P. Thiessen's master thesis [123], another method has been developed. We went a step back to equation (3.10) and further modified this equation oriented at a publication of Borca-Tasciuc et al. in 2001 [124]. While equation (3.10) needs an infinite half-space, which means in practice a substrate thickness of $t_{s} \gg\left|\frac{1}{q}\right|$, the equation has been modified for finite substrate thicknesses implementing a backside boundary condition. Experimentally we found that the adiabatic model fits best to our results. The modification of equation (3.10) for a substrate with thickness $t_{s}$ and thermal anisotropy $\eta_{s}$, using an adiabatic backside boundary condition, is presented in equation (3.12). The measured $\Delta T$ on the left side of the equation was fitted to the modulus (because what physically is measured is actually the modulus of a quantity) of the whole integral plus a contribution $\Delta T_{\text {tot }}$ by film, thermal boundary resistances and (if necessary) an insulation layer. $\Delta T_{\text {tot }}$ is simply added implementing the so called offset model which will be discussed below.

$$
\Delta T=\left|\frac{P}{l \pi \kappa_{s, y}} \int_{0}^{\infty} \frac{\sin ^{2}(k b)}{\tanh \left(t_{s} \sqrt{\eta_{s} k^{2}+q^{2}}\right)(k b)^{2} \sqrt{\eta_{s} k^{2}+q^{2}}} d k+\Delta T_{t o t}\right|
$$




\subsubsection{The offset model}

For sufficiently low film thicknesses, the thermal offset caused by the film can be considered to be constant and frequency-independent. The offset can be seen as a thermal resistance in analogy to an electrical series resistant. The boundary conditions that have to be fulfilled for the offset-model are a small film thickness compared to the heater width (equation (3.13)) and a much smaller thermal conductivity of the film compared to that of the substrate (equation (3.14)).

$$
\begin{aligned}
& t_{f} \ll 2 b \\
& \kappa_{f} \ll \kappa_{s}
\end{aligned}
$$

When these conditions are fulfilled, an approximately perpendicular heat flow through the film can be assumed and the addition of the offset $\Delta T_{\text {tot }}$ in equation (3.12) is justified. Precision has been further improved by using a corrected or effective heater width $2 b_{e f f}=2 b+0.76 t_{f} \sqrt{\eta_{f}}$ [124] taking into account lateral heat flow through the film. Here, $\eta_{f}$ is the thermal anisotropy of the film. Using this correction, one has to replace $2 b$ by $2 b_{e f f}$ in the respective equations.

The offset model was presented for the first time by Cahill et al. [121], whereby the relation between thermal offset of a film $\Delta T_{f}$ and its thermal conductivity perpendicular to the surface $\kappa_{f, y}$ was simply expressed by Fourier's law:

$$
\Delta T_{f}=\frac{P}{l \kappa_{f, y}} \frac{t_{f}}{2 b}
$$

Equation (3.15) works very well especially for poor conducting and isotropic films. For films approaching the thermal conductivity of the substrate and/or for anisotropic films, a modified offset model furnishes more accurate results.

\section{The modified offset model}

Tong et al. [125] presented a modified offset model which was partially adopted [123] and subsequently used for the evaluation routine obtaining the results presented in this thesis. The derivation based on ref. [125] is shown here.

Starting with equation (3.15) a correction term $\Gamma$ and the thermal resistance between substrate and film $R_{f, s}$ have been added. As result a total thermal offset by film and thermal boundary resistance between film and substrate is shown in equation (3.16).

$$
\Delta T_{f+f, s}=\frac{P}{2 b l}\left[\frac{t_{f}}{\kappa_{f, y}} \Gamma\left(K, B i_{f}, H r\right)+R_{f, s}\right]
$$




$$
K=\frac{\kappa_{f, y}}{\kappa_{s, y}} \quad B i_{f}=\frac{t_{f}}{\kappa_{f, y} R_{K}} \quad H r=\frac{b}{t_{f} \sqrt{\eta_{f}}}
$$

$R_{K}$ is the sum of all thermal boundary resistances in the system, also called Kapitza resistances, while the added $R_{f, s}$ term only includes the boundary resistance between substrate and film. We replaced $R_{f, s}$ by $R_{K}$ and additionally added $R_{\mathrm{SiO}_{2}}$, the thermal resistance contribution by the insulation layer (amorphous $\mathrm{SiO}_{2}$, necessary for the $3 \omega$ measurement of electrically conductive film materials such as $\left.\left(\mathrm{Sb}_{1-\mathrm{x}} \mathrm{Bi}_{\mathrm{x}}\right)_{2} \mathrm{Te}_{3}\right)$. The thermal offset by Kapitza resistances and insulation layer is therefore given by $\Delta T_{K, I}=\frac{P}{l 2 b}\left(R_{K}+R_{\mathrm{SiO}_{2}}\right)$. With interim step (3.18) this leads to equation (3.19) for the thermal offset caused by only the film $\Delta T_{f}$.

$$
\begin{gathered}
\Delta T_{t o t}=\frac{P}{2 b l}\left[\frac{t_{f}}{\kappa_{f, y}} \Gamma\left(K, B i_{f}, H r\right)+R_{K}+R_{\mathrm{SiO}_{2}}\right] \\
\Delta T_{f}=\Delta T_{t o t}-\Delta T_{K, I}=\frac{P t_{f}}{2 b l \kappa_{f, y}} \Gamma\left(K, B i_{f}, H r\right)
\end{gathered}
$$

For the next step, Tong et al. assumed the conditions shown in (3.20).

$$
t_{s} \gg\left|\frac{1}{q}\right| \quad, \quad t_{f} \ll\left|\frac{1}{q}\right| \quad, \quad t_{f} \ll 2 b
$$

Tong et al. subsequently derived equation (3.21) for the thermal impedance $\Delta Z$ and the Kapitza resistance between substrate and film in Fourier space:

$$
\Delta Z=\frac{t_{f}}{\kappa_{f, y}}\left(1-\frac{\kappa_{f, y}^{2} \eta_{f}}{\kappa_{s, y}^{2} \eta_{s}}-\frac{2 R_{f, s} \kappa_{f, y}^{2} \eta_{f} \lambda}{\kappa_{s, y} \sqrt{\eta_{s}}}-R_{f, s}^{2} \kappa_{f, y}^{2} \eta_{f} \lambda^{2}\right)+R_{f, s}
$$

Here, $\lambda$ is the Fourier transform variable in $\mathrm{x}$ direction. It denotes the $\mathrm{x}$-component of the spatial frequency (wave vector) and its physically important range can be estimated by the reciprocal heater width $\frac{1}{2 b}$. Thereby and taking the Kapitza B resistances published in [84] as an upper limit for $R_{f, s}$, it can be shown that the third and fourth term within the bracket of equation (3.21) are negligible for the performed $3 \omega$ measurements of $\left(\mathrm{Sb}_{1-\mathrm{x}} \mathrm{Bi}_{\mathrm{x}}\right)_{2} \mathrm{Te}_{3}$ films. This leads to a correction term $\Gamma$ shown in equation (3.22).

$$
\Gamma=1-\frac{\kappa_{f, y}^{2} \eta_{f}}{\kappa_{s, y}^{2} \eta_{s}}
$$

The combination of equations (3.19) and (3.22) yields the expression (3.23) as link between the thermal offset of the film $\Delta T_{f}$ and the crossplane component of the thermal conductivity of the film $\kappa_{f, y}$.

$$
\Delta T_{f}=\frac{P t_{f}}{2 b l \kappa_{f, y}}\left(1-\frac{\kappa_{f, y}^{2} \eta_{f}}{\kappa_{s, y}^{2} \eta_{s}}\right)
$$

Finally, transposing of equation (3.23) reveals equations (3.24) and (3.25) which finally have been used for the determination of $\kappa_{f, y}$. 


$$
\begin{gathered}
\kappa_{f, y}=\sqrt{\frac{1}{4} \Xi^{2}+\frac{\eta_{s}}{\eta_{f}} \cdot \kappa_{s, y}^{2}}-\frac{1}{2} \Xi \\
\Xi=\frac{2 b l}{P t_{f}} \cdot \frac{\eta_{s}}{\eta_{f}} \cdot \kappa_{s, y}^{2} \cdot \Delta T_{f}
\end{gathered}
$$

The big advantage of the modfied offset model consists in consideration of both the film anisotropy and the ratio of substrate and film thermal conductivities. Equations (3.24) and (3.25) enable the handling of substrate thermal conductivities which are close to the film thermal conductivities. By that it significantly increases the number of possible substrate-film combinations. It also results in an expansion of the measurement range to higher temperatures. In particular the application of these refined formulas increases the measurement accuracy and data reliability.

\subsubsection{Implementing the evaluation process}

The concrete evaluation was done by a script written in the program MATLAB (MathWorks $\left.{ }^{\circledR}\right)$. For every single temperature, $\Delta T$ has been calculated according to equation (3.5), one value for every frequency. Fitting the right side of equation (3.12) to the measured $\Delta T$ values has been done using $\kappa_{s, y}$ and $\Delta T_{t o t}$ as fit parameters. For $\eta_{s}$ a value of 0.93 for the $\alpha-\mathrm{Al}_{2} \mathrm{O}_{3}$ substrates has been used. All other parameters have been taken either from literature (the temperature dependency of $C\left(\mathrm{Al}_{2} \mathrm{O}_{3}\right)$ is taken from Ditmars et al. [126]) or have been measured via SEM, TEM or profilometry.

The desired $\kappa_{f, y}$ has been determined in a next step by using equations (3.24) and (3.25).

For error calculation, initially for every single quantity an error has been estimated. Subsequently, the evaluation routine described above has been performed for systematically composed combinations of deviations of the quantities within their error ranges. The standard deviations $\sigma$ have been calculated out of the so obtained distributions for $\kappa_{s, y}$ and $\kappa_{f, y}$ values. The finally declared errors have been determined using a confidence interval of $2 \sigma$.

\subsubsection{The experimental setup}

The $3 \omega$ measurements have been carried out at a self-built setup. Two bachelor $[127,128]$ and one diploma thesis [129] have contributed to the development and optimization of the system. The setup is designed on basis of the doctoral thesis of C. E. Raudzis [130], which provides more details about the concept of the measurement setup. The following list shows the technical equipment which has been used, all self-built components have been developed and constructed in cooperation with the electronic workshop of the Institute of Material Physics:

- Anfatec eLockIn 204/2: Heart of the $3 \omega$ measurement device is a Lock-In amplifier. This powerful model produced by the company Anfatec is used as voltage 
source, an AC voltage with angular frequency $\omega$ is applied by the output port. Its quantity is well adjusted to the before measured resistances of supply lines and $\mathrm{Pt}$ heater in order to apply a well defined electrical power to the Pt heater.

The input signal of the Lock-In amplifier is a preamplified signal consisting of an $\omega$ and a $3 \omega$ part. The amplitude of $U_{3 \omega}$ lies in the range of $<\mathrm{mV}$ and it has to be divided from a still much bigger $\omega$ signal, however, the precise determination of $U_{3 \omega}$ is indispensable. This task only can be carried out by a high-performance Lock-In amplifier.

Furthermore the phase shift between outgoing $\omega$ signal and incoming $3 \omega$ signal is measured. A switch elsewhere implemented in the setup enables the measurement of the phase shift created by the whole system without the Pt heater itself. These phase determinations have been performed during every measurement for the sake of completeness, however, they have not been used for the results presented in this thesis due to the application of the improved evaluation routine using equation (3.12).

- Driver circuit (self-built): The active driver circuit receives the outgoing signal of the Lock-In amplifier and possesses two outputs. The signal is forwarded to the sample (Pt heater) and to the so called resistance decade.

- Resistance decade (self-built): This component is used for "mirroring" the resistance of the $\mathrm{Pt}$ heater. The outgoing signals from both resistance decade and Pt heater are supposed to have a very similar $\omega$ component. Therefore the resistance decade signal is subtracted in a preamplification process to support the work of the Lock-In amplifier.

- Bridge (self-built): The so called "bridge" constitutes a kind of center of the setup. It connects many components and enables measurement and subtraction processes. Since it is no active component, it has neither an own power supply nor an interface with the measurement PC.

- Agilent Technologies L4532A $20 \mathrm{MSa} / \mathrm{s}$ Digitizer: On the one hand, this A-D converter is used to measure precisely the $U_{\omega}$ voltage dropping off over the $\mathrm{Pt}$ heater. On the other hand it is capable of performing a Fast Fourier Transformation (FFT) of the (preamplified) signal. The latter function is useful in terms of checking for interfering signals pointing out to possible measurement problems.

- Stanford Research Systems Model SR560 Low-Noise-Preamplifier: The preamplifier subtracts the signal gripped at the resistance decade from the signal of the $\mathrm{Pt}$ heater. The resulting signal has an $\omega$ to $3 \omega$ ratio which is orders of magnitudes lower than the ratio for the original sample signal. This crucial preamplification supports the Lock-In amplifier in the precise measurement of $U_{3 \omega}$.

- Keithley 2000 Multimeter: This multimeter is used for the measurement of the voltage drop over a so called Shunt resistance, which has a well known electrical resistance. Since Shunt resistance and Pt heater are connected in series, this enables 
to calculate the current through both. In combination with the measurement of the voltage drop over the Pt heater, the Pt heater resistance is determined.

- Keithley 2700 Multimeter / Data Acquisition System: This component measures the voltage which drops over the resistance decade.

The measurements have been carried out in a closed cycle cryostat under high vacuum conditions. Beyond the actual $3 \omega$ measurement setup, additional devices listed hereafter were in use in order to establish the desired measurement conditions:

- Pfeiffer DUO 5 M: A rotary vane pump is used as a backing pump in order to support the turbomolecular pump.

- Pfeiffer TVM 261: This turbomolecular pump (1000 Hz, $\left.\mathrm{S}\left(\mathrm{N}_{2}\right)=210 \frac{1}{\mathrm{~s}}\right)$ kept the cryostat under high vacuum conditions in order to prevent unwanted thermal transport processes to or from the sample. A proper vacuum is also absolutely necessary for the prevention of condensating humidity.

- Leybold RW-2 helium compressor: This water cooled compressor works with helium gas under high pressures. Temperatures down to approximately $20 \mathrm{~K}$ can be achieved. The main advantages compared to systems which use cryogenic liquefied gases consist in lower costs and easier operation.

- Leybold Temperature Controller LTC 60: While the compressor always works on maximum power, temperature is controlled by this PID controller. To ensure the thermalization throughout the copper finger and the sample fixed on the latter by vacuum grease, at every temperature step a hold time of usually $1200 \mathrm{~s}$ has been complied. Systematical experimental variation of the hold time indicated that thermalization has taken place after the mentioned period. Application of the $\mathrm{Pt}$ heater resistance vs. temperature measured by the LTC 60 provides a very linear slope, confirming this assumption.

- Knick Präzisionsstromgeber: A diode is placed at the underside of the copper finger and can be used for further temperature measurements. This device works as a current source for the diode.

- Keithley 199: This instrument determines the voltage at the diode. Via the characteristic $R(T)$ curve the measured resistances provide temperature values. 


\subsection{TEM lamella preparation by Focused Ion Beam}

HRTEM used to determine the microstructure at the atomic scale requires a special preparation process. This preparation was done in a Scanning Electron Microscope (SEM) equipped with a Ga-ion source, a so called Focused Ion Beam (FIB). The used device is a Nova NanoLab 600 (Thermo Fisher Scientific).

A good overview about the general functioning of a FIB can be found in a review paper by Ali et al. [131].
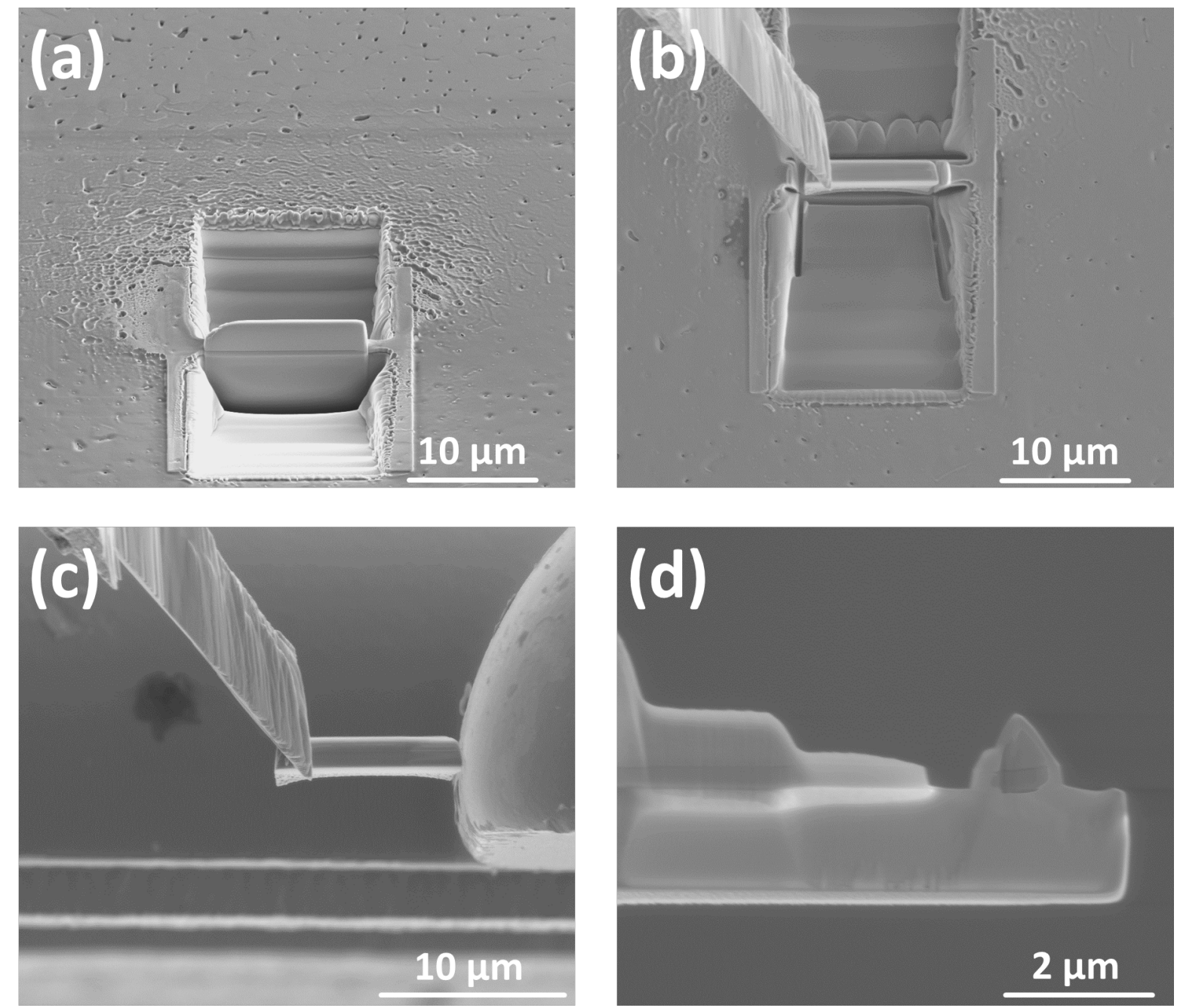

Figure 3.1: Different steps of the TEM lamella preparation of a $\left(\mathrm{Sb}_{0.88} \mathrm{Bi}_{0.12}\right)_{2} \mathrm{Te}_{3}$ film on an $\alpha-\mathrm{Al}_{2} \mathrm{O}_{3}$ substrate by means of a Focused Ion Beam (FIB) device.

In this section I want to comment on the used routine for preparing so called lamellas which subsequently were investigated by HRTEM. Figure 3.1 shows SEM images illustrating different steps of the TEM lamella preparation process. 
The first steps of the sample preparation were straight forward oriented at established proceedings. First of all a protection layer of Pt-C has been deposited by Electron-beam assisted platinum deposition (EBAD) at an acceleration voltage of $5 \mathrm{kV}$ and a beam current of $1.6 \mathrm{nA}$. Subsequently a thicker Pt-C protection layer has been deposited via Ion-beam assisted platinum deposition(IBAD). This was followed by different cutting steps until the lamella stood free (Figure 3.1 (a)).

The next steps were the so called $U$ cut and the attachment of the lamella to an inserted piezo element controlled micromanipulator, the so called Omniprobe, by Pt-C (Figure 3.1 (b)).

After removing the last connection between lamella and substrate/film and a careful liftout process, the lamella was transferred to a $\mathrm{Cu}$ grid (Figure 3.1 (c)) to which it was attached by $\mathrm{Pt}-\mathrm{C}$ from two sides. The special lateral mounting at one end of the lamella was chosen to reduce mechanical stress and strain.

At the ion source an acceleration voltage of $30 \mathrm{kV}$ was used in combination with different ion beam currents. The ranges of those beam currents were $10 \mathrm{pA}$ to $300 \mathrm{pA}$ for the deposition of $\mathrm{Pt}-\mathrm{C}$ and $100 \mathrm{pA}$ to $7 \mathrm{nA}$ for cutting and thinning purposes.

The most challenging part consists in the last thinning step. Conventionally it is done at $30 \mathrm{kV}$ and $10 \mathrm{pA}$ and proper results can be achieved with that parameters. However, the $\left(\mathrm{Sb}_{1-\mathrm{x}} \mathrm{Bi}_{\mathrm{x}}\right)_{2} \mathrm{Te}_{3}$ films turned out to be very sensitive to the ion beam which means a high danger of knock-on damage. This can be explained by the weak bondings mainly between the van der Waals bonded quintuple layers. Knock-on damage leads to thick amorphous layers at the surfaces of the lamella reducing the quality of the achievable HRTEM images.

The solution developed by the author consisted in applicating a reduced ion acceleration voltage of $5 \mathrm{kV}$ with the lowest possible current at that voltage which was $29 \mathrm{pA}$. In this way it was possible to reduce the energy of each ion by a factor of 6 . A side view from the lamella after this last thinning step is shown in Figure 3.1 (d).

\subsection{High Resolution Transmission Electron Microscopy}

A crucial part of my work was the analysis of the microstructure of the films by Transmission Electron Microscopy (TEM). TEM lamellas have been prepared via FIB, partially followed by $\mathrm{Ar}^{+}$ion milling in a Precise Ion Polishing System (PIPS) at voltages below $1 \mathrm{kV}$. Different TEMs have been used for precharacterization of the lamellas: A CM12 $(120 \mathrm{kV})$ equipped with an EDX detector, a CM120 (120kV) and a CM300 $(300 \mathrm{kV})($ all $F E I)$.

For further structural studies High Resolution TEM (HRTEM) at a Thermo Fisher Scientific Titan $80-300 \mathrm{kV}$ operated at $300 \mathrm{kV}$ has been applied. Immediately before the insertion of the lamellas into the microscope, a further plasma cleaning step (75\% Argon - $25 \%$ Oxygen mixture) has been conducted. The used TITAN electron microscope is equipped with a $C_{\mathrm{S}}$ corrector, a magnetic hexapole which is placed beneath the sample. 
Amongst other image errors, the spherical aberration $C_{\mathrm{S}}$ is strongly mitigated by this corrector. As a result, very high magnifications down to atomic resolution are possible: For the lateral HRTEM resolution $0.8 \AA$ are specified.

Besides HRTEM, the used operation modes at the TITAN comprised also Scanning Transmission Electron Microscopy (STEM). In STEM, the distinction of different elements is possible due to their different atomic numbers affecting the strength of electron scattering. Therefore a so called High Angle Annular Dark Field (HAADF) detector has been used. For the investigation of substrate-film interfaces, Electron Energy Loss Spectroscopy (EELS) has been applied.

An important step was the development of a customized lamella preparation procedure as mentioned in the previous section. The difference between $30 \mathrm{kV}$ and $5 \mathrm{kV}$ at the last thinning step is remarkable and catches the eye, see figure 3.2.
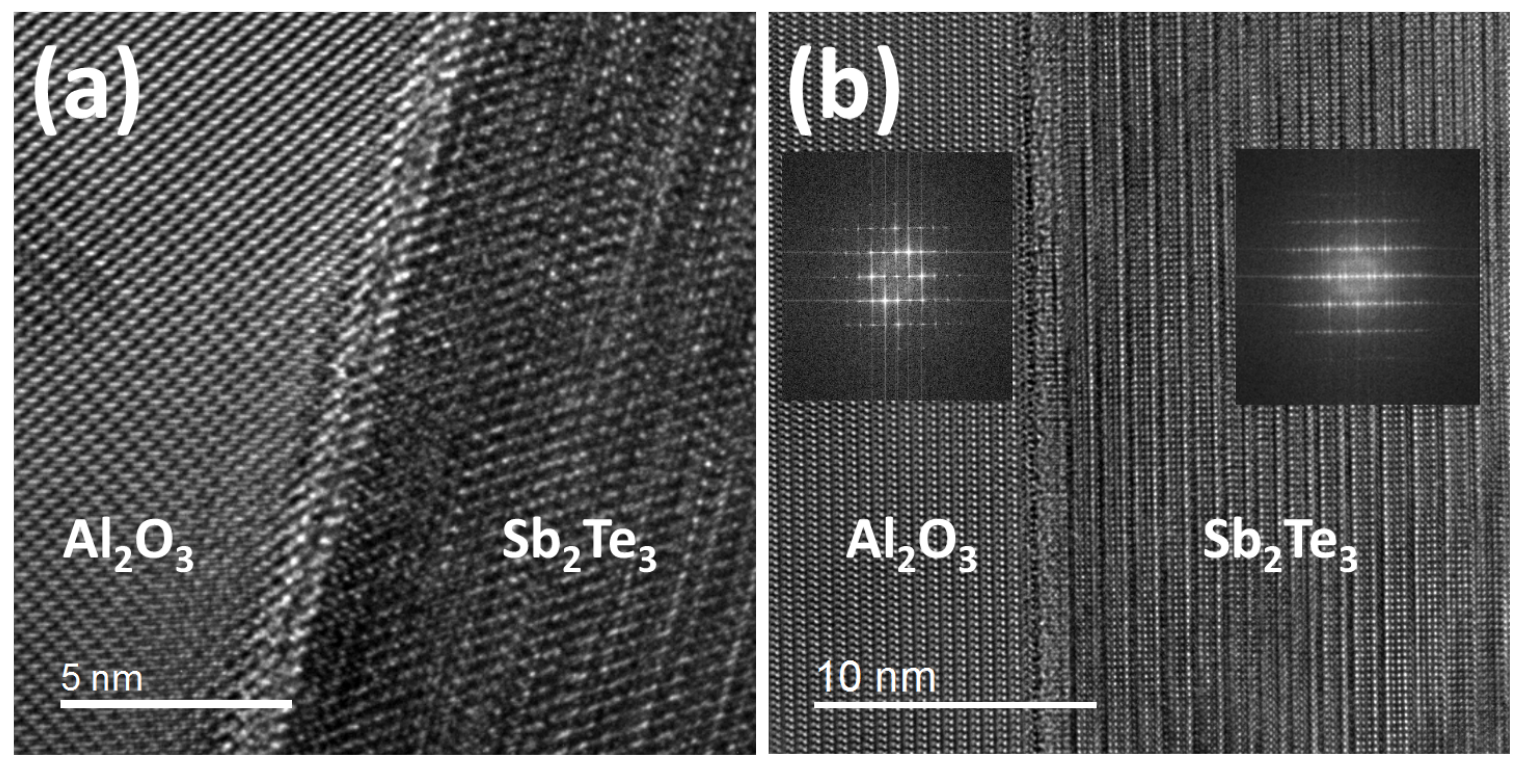

Figure 3.2: HRTEM image of an $\alpha-\mathrm{Al}_{2} \mathrm{O}_{3}-\mathrm{Sb}_{2} \mathrm{Te}_{3}$ interface with last FIB thinning step at (a) $30 \mathrm{kV}$ and (b) $5 \mathrm{kV}$. The included Fast Fourier Transformations (FFTs) at (b) prove the epitaxial growth of the film.

(b) is a modification of figure 7(a) in F. Rieger, K. Kaiser, G. Bendt, V. Roddatis, P. Thiessen, S. Schulz, and C. Jooss, J. Appl. Phys. 123:175108, 2018. DOI: $10.1063 / 1.5025491$ [84]. Used here in accordance with the CC BY 4.0 license

Exact construction, design and operating principles of modern TEMs are very complex. They can be found elsewhere $[132,133]$ and will not be described in detail at this point. Concerning $C_{\mathrm{S}}$ corrected TEM and STEM, a report about the use of a hexapole corrector provides further information [134] and general considerations about the resolution limits of HRTEM are stated in an article by David J. Smith [135]. 


\section{CHAPTER 4}

\section{Low intrinsic c-axis thermal conductivity in PVD grown epitaxial $\mathrm{Sb}_{2} \mathrm{Te}_{3}$ films}

This chapter consists of the accepted manuscript, including the Supplemental Information, of the original article

Low intrinsic c-axis thermal conductivity in PVD grown epitaxial $\mathrm{Sb}_{2} \mathrm{Te}_{3}$ films F. Rieger ${ }^{1}$, K. Kaiser ${ }^{2}$, G. Bendt ${ }^{2}$, V. Roddatis ${ }^{1}$, P. Thiessen ${ }^{1}$, S. Schulz ${ }^{2}$, and C. Jooss ${ }^{1}$

${ }^{1}$ Institute of Material Physics, University of Göttingen, 3707r Göttingen, Germany

${ }^{2}$ Institute of Inorganic Chemistry and Center for Nanointegration Duisburg-Essen (Cenide), University of Duisburg - Essen, 45141 Essen, Germany

Originally published in Journal of Applied Physics 123:175108, 2018. [84]

Published under the CC BY 4.0 license.

DOI: $10.1063 / 1.5025491$

The manuscript has been submitted without arrangement of figures.

The arrangement of figures (changed in size) shown here has been done by the author of this thesis, oriented at the published article.

Minor changes have been made by correcting misspellings and by changing font sizes and line spacing. In the Introduction "Reduction of thermal conductivity" has been corrected to "Reduction of lattice thermal conductivity", references 1 and 2 have been corrected and in section I. of the Supplemental Information the indices of the thermal conductivities have been aligned for the sake of consistency. 



\title{
Low intrinsic c-axis thermal conductivity in PVD grown epitaxial $\mathrm{Sb}_{2} \mathrm{Te}_{3}$ films
}

\author{
F. Rieger, ${ }^{1}$ K. Kaiser, ${ }^{2}$ G. Bendt, ${ }^{2}$ V. Roddatis, ${ }^{1}$ P. Thiessen, ${ }^{1}$ S. Schulz, ${ }^{2}$ \\ and C. Jooss ${ }^{1}$ \\ ${ }^{1}$ Institute of Material Physics, University of Göttingen, 37077 Göttingen, Germany \\ ${ }^{2}$ Institute of Inorganic Chemistry and Center for Nanointegration Duisburg-Essen \\ (Cenide), University of Duisburg - Essen, 45141 Essen, Germany
}

Accurate determination and comprehensive understanding of the intrinsic c-axis thermal conductivity $\kappa_{c}$ of thermoelectric layered $\mathrm{Sb}_{2} \mathrm{Te}_{3}$ is of high importance for the development of strategies to optimize the figure of merit in thin film devices via heterostructures and defect engineering. We present here high precision measurements of $\kappa_{c}$ of epitaxial $\mathrm{Sb}_{2} \mathrm{Te}_{3}$ thin films on $\mathrm{Al}_{2} \mathrm{O}_{3}$ substrates grown by physical vapor deposition (PVD) in the temperature range of $100 \mathrm{~K}$ to $300 \mathrm{~K}$. The Kapitza resistances of the involved interfaces have been determined and subtracted from the film data, allowing access to the intrinsic thermal conductivity of single crystalline $\mathrm{Sb}_{2} \mathrm{Te}_{3}$. At room temperature, we obtain $\kappa_{c}=1.9 \mathrm{~W} / \mathrm{mK}$, being much smaller than the in-plane thermal conductivity of $\kappa_{a b}=5 \mathrm{~W} / \mathrm{mK}$ and even lower than the thermal conductivity of nanocrystalline films of $\kappa_{\mathrm{nc}} \approx 2.0-2.6 \mathrm{~W} / \mathrm{mK}$ published by Park et al. ${ }^{1}$. High crystallinity and very low defect concentration of the films were confirmed by x-ray diffraction (XRD) and high resolution transmission electron microscopy (HRTEM). Our data reveal that the phonon mean free path $l_{m f p}(T)$ is not limited by defect scattering and is of intrinsic nature, i.e. due to phononphonon scattering similar to other soft van der Waals type bonded layered systems.

\section{INTRODUCTION}

Tetradymite-type materials such as $\mathrm{Sb}_{2} \mathrm{Te}_{3}$ and $\mathrm{Bi}_{2} \mathrm{Te}_{3}$ are thermoelectric materials with promising high figure of merit ZT close to 1 in a temperature range of 300-400 $\mathrm{K}$ which is promising for technical applications ${ }^{2}$. The thermoelectric efficiency of a material is given by the dimensionless figure of merit $Z T=\left(S^{2} \sigma / \kappa\right) T(S=$ Seebeck coefficient, $\sigma=$ specific electrical conductivity, $\kappa=$ thermal conductivity $=$ sum of electronic $\kappa_{\mathrm{el}}$ and lattice $\kappa_{\mathrm{la}}$ contributions, $\mathrm{T}=$ absolute temperature $[\mathrm{K}])^{3}$. In order to achieve a high ZT, high Seebeck coefficients (S), high electrical conductivity $(\sigma)$ and low thermal conductivity $(\kappa)$ are required. For many thermoelectric relevant materials the lowering of lattice thermal conductivity by the introduction of grain boundaries or interfaces in heterostructures has been demonstrated ${ }^{4}$. Reduction of lattice thermal conductivity down to $\kappa=0.22 \mathrm{~W} / \mathrm{mK}$ by a factor of 7-8 was reported for $\mathrm{Sb}_{2} \mathrm{Te}_{3} / \mathrm{Bi}_{2} \mathrm{Te}_{3}$ 
multilayer structures by Venkatasubramanian et al. ${ }^{5}$. The same author claimed a high ZT value of 2.4 for p-type $\mathrm{Sb}_{2} \mathrm{Te}_{3} / \mathrm{Bi}_{2} \mathrm{Te}_{3}$ multilayer structures ${ }^{6}$. This result could not be reproduced by other research groups and was later questioned since the superlattices have been shown to be unstable at elevated temperatures ${ }^{7,8,9}$. Here, reduced cross-plane thermal conductivity ( 0.55 to $0.6 \mathrm{~W} / \mathrm{mK}$ ) due to nanoalloying of the superlattice at elevated temperatures was observed, while no measurable reduction of the thermal conductivity by the superlattice-type 2D nanostructuring was found (see also the overview in ref. $^{8}$ ), therefore pointing against simultaneous low thermal and high electrical cross plane conductivities in such systems.

High-quality $\mathrm{Sb}_{2} \mathrm{Te}_{3}$ films have been grown by use of different methods, including molecular beam epitaxy (MBE) ${ }^{10,11,12}$ RF-magnetron sputtering, ${ }^{13}$ pulsed laser deposition (PLD) ${ }^{14,15}$ metal organic chemical vapor deposition (MOCVD) ${ }^{16,17}$ and thermal (co-)evaporation, ${ }^{18,19,20,21}$ respectively. ${ }^{22,23}$ Among these, thermal (co-)evaporation is of particular interest, since it allows the deposition of highly stoichiometric, impurity-free $\mathrm{Sb}_{2} \mathrm{Te}_{3}$ thin films on rather large substrates with high growth rates starting with commercially available, high-purity $\mathrm{Sb}_{2} \mathrm{Te}_{3}$ powder. As a consequence, it is a far less expensive and less time-consuming method compared to MOCVD, MBE and sputtering, but to the best of our knowledge, its capability for the epitaxial growth of highly c-oriented $\mathrm{Sb}_{2} \mathrm{Te}_{3}$ films has not been demonstrated.

For an understanding of the thermal conductivity in superlattices or nanocrystalline materials, reliable data of the anisotropic intrinsic thermal conductivity of single crystalline tellurides is required. Whereas temperature dependent data for the in-plane thermal conductivity $\kappa_{a b}$ in $\mathrm{Sb}_{2} \mathrm{Te}_{3}$ single crystals is published ${ }^{24,25}$, to the best of our knowledge, data on c-axis thermal conductivity $\kappa_{\mathrm{c}}$ is only reported for room temperature ${ }^{26}$. Park et al. ${ }^{1}$ measured the thermal conductivity of magnetron-sputtered nanocrystalline $\mathrm{Sb}_{2} \mathrm{Te}_{3}$ films, where the grain size has been varied by annealing. Remarkably, the room temperature thermal conductivity $\kappa_{a b}=5 \mathrm{~W} / \mathrm{mK}$, $\kappa_{\mathrm{c}}=1.9 \mathrm{~W} / \mathrm{mK}$ shows a pronounced anisotropy $A=\kappa_{a b} / \kappa_{c} \approx 2.6$. Consequently, the thermal conductivity in nanocrystalline films in the range of $\kappa_{\mathrm{nc}} \approx 2.0-2.6 \mathrm{~W} / \mathrm{mK}$ is certainly strongly influenced by the anisotropy. Temperature dependent $\kappa_{c}(T)$ measurements are thus highly desirable for the understanding of nanostructured tellurides and for basic insights into the transport mechanism of phonons across layered systems which are connected only via van der Waals bonding. Furthermore, for the application in thermoelectric devices the c-axis component $\kappa_{\mathrm{c}}(\mathrm{T})$ is most relevant.

Here, we present $\kappa_{c}(T)$ in the range between $T=100-300 \mathrm{~K}$ determined by the $3 \omega$ method. Highly crystalline epitaxial $\mathrm{Sb}_{2} \mathrm{Te}_{3}$ films with a very low defect concentration have been grown by physical vapor deposition (PVD). The epitaxial growth and crystallinity is confirmed by $\mathrm{x}-$ ray diffractometry as well as by transmission electron microscopy (TEM). High resolution transmission electron microscopy (HRTEM) shows a low density of stacking faults and grain boundaries. In order to determine the intrinsic $\kappa_{\mathrm{c}}(\mathrm{T})$, a careful determination and subtraction of 
the Kapitza resistances of all involved interfaces between the $\mathrm{Sb}_{2} \mathrm{Te}_{3}$ films and the substrate as well as the $3 \omega$ heater structure is required. This is carried out by variation of the thickness of the $\mathrm{Sb}_{2} \mathrm{Te}_{3}$ films as well as of the $\mathrm{SiO}_{2}$ layer, required for electrical insulation of the Pt heater structures from the thermoelectric films in the $3 \omega$ measurements. The paper is organized as follows: In the experimental method section, we present the growth and characterization of the PVD $\mathrm{Sb}_{2} \mathrm{Te}_{3}$ films as well as the introduction of the used $3 \omega$ setup. We continue in presenting the thermal conductivity measurements and the HRTEM characterization. In the discussion section, the obtained intrinsic $\kappa_{c}(T)$ of the epitaxial films is compared with literature data of single crystalline and nanocrystalline $\mathrm{Sb}_{2} \mathrm{Te}_{3}$ samples, and the determined phonon mean free path is discussed in the light of the determined crystal- and microstructure. This allows for conclusions on the intrinsic limitation of the c-axis phonon thermal conductivity in the van der Waals type layered system.

\section{EXPERIMENTAL}

\section{A. Film preparation via PVD}

Epitaxial thin films of $\mathrm{Sb}_{2} \mathrm{Te}_{3}$ were grown on $10 \mathrm{x} 10 \mathrm{~mm}$-oriented sapphire $\left(\mathrm{Al}_{2} \mathrm{O}_{3}(0001)\right)$ substrates in a vertical PVD-reactor at a substrate temperature of $330{ }^{\circ} \mathrm{C}$. Commercial $\mathrm{Sb}_{2} \mathrm{Te}_{3}$ powder (150 mg, $99.999 \%$ abcr) was evaporated at $570{ }^{\circ} \mathrm{C}$ at 20 mbar within $15 \mathrm{~min}$.

According to XPS studies (FIG. S1), the $\mathrm{Sb}_{2} \mathrm{Te}_{3}$ films show a thin (1-3 nm) oxide surface layer due to post-oxidation processes during transport and handling of the films under atmosphere. However, the thickness of the oxide layer did not increase with time as was shown by analyzing a sample that was stored for 1 month under ambient conditions.

A Bruker D8 Advance powder diffractometer in a Bragg-Brentano geometry with $\mathrm{Cu}-\mathrm{K} \alpha$ radiation $(\lambda=1.5418 \AA$ ) was used for XRD studies. A typical XRD pattern as displayed in FIG. 1 shows the characteristic $00 l(l=3,6,9,12, \ldots)$ reflexions for $c$-oriented antimony telluride (PDF: 71-393). No other crystalline phases are present.

SEM investigations done at a FEI Nova NanoSEM 650 shown in FIG. 2 reveal a flat surface morphology. All films show island growth mode. Crystallites of some $\mu \mathrm{m}$ size are observed which are all c-oriented as confirmed by XRD and TEM. Flatness is an important requirement for depositing a proper insulation layer and Pt heater structures for reliable $3 \omega$ measurements. 


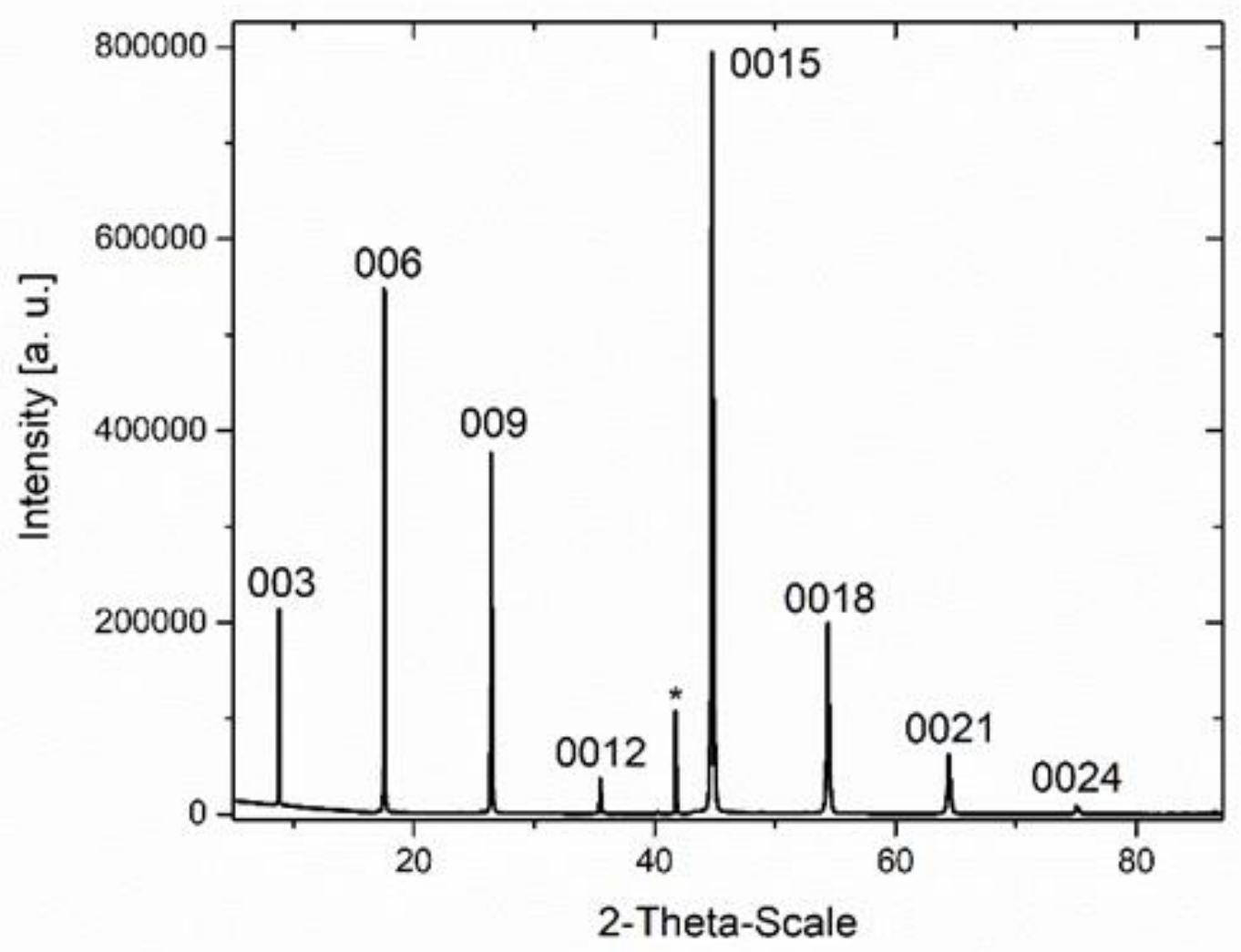

FIG. 1. X-ray diffractogram of a PVD grown $\mathrm{Sb}_{2} \mathrm{Te}_{3}$ film on a $c$-oriented $\mathrm{Al}_{2} \mathrm{O}_{3}$ substrate. The asterisk corresponds to the 006 reflection of the $\mathrm{Al}_{2} \mathrm{O}_{3}$ substrate. 


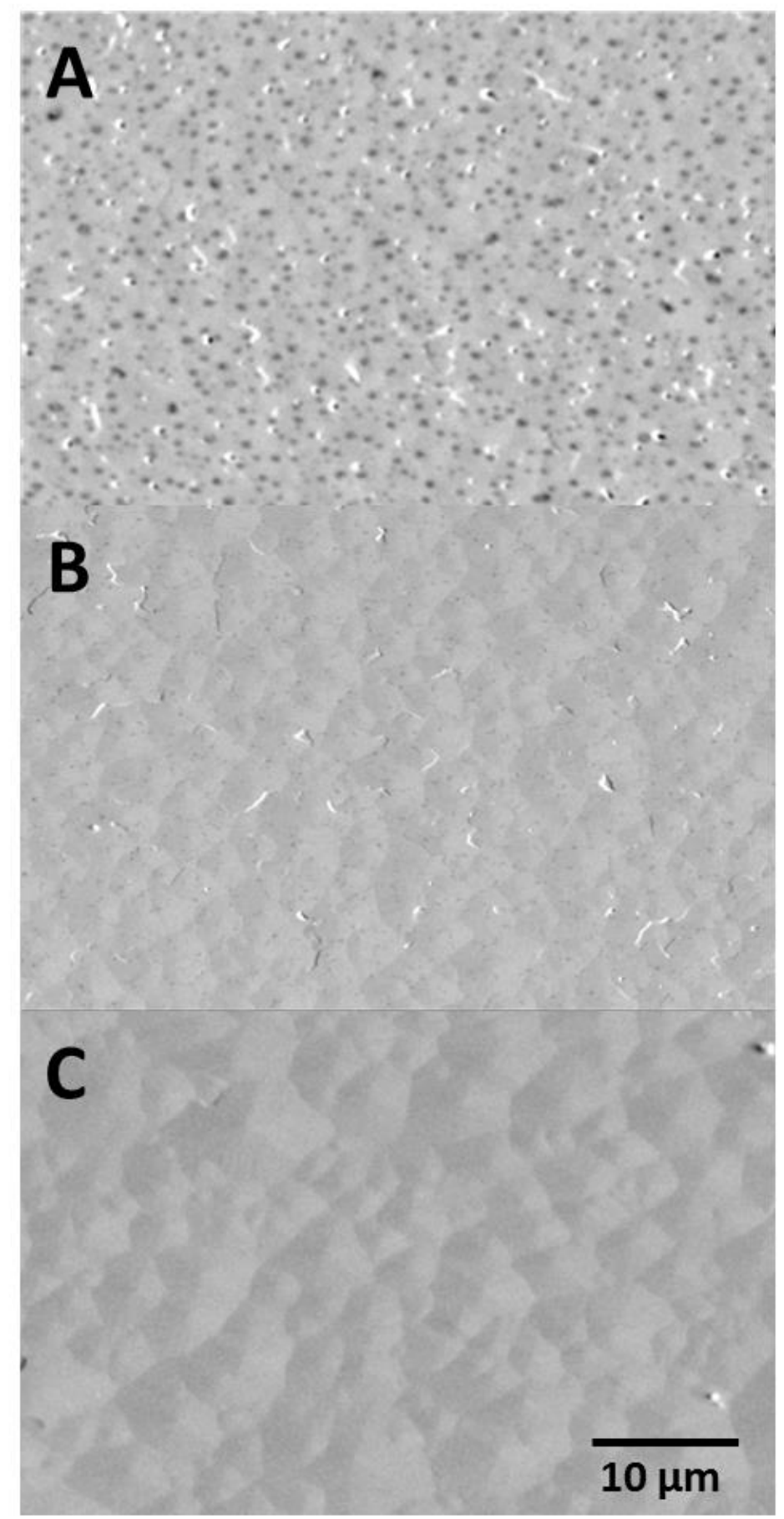

FIG. 2. SEM images of $\mathrm{Sb}_{2} \mathrm{Te}_{3}$ samples with different thicknesses taken under 45 degree inclination, (A) 191, (B) 487, and (C) $1184 \mathrm{~nm}$. The dark contrast in FIG. 2A for the thinnest film is most probable due to pinholes. 


\section{B. High resolution TEM}

TEM lamellas have been prepared via Focused Ion Beam (FIB) technology using a Nova NanoLab 600 (FEI, Netherlands), operated at $30 \mathrm{kV}$ and $5 \mathrm{kV}$, and partially followed by a cleaning argon ion milling step in a Gatan PIPS. HRTEM, STEM and EELS studies have been performed at a $C_{\mathrm{S}}$ corrected FEI Titan $80-300 \mathrm{kV}$ operated at $300 \mathrm{kV}$.

\section{Sample geometry for thermal conductivity measurements}

The geometry of the samples including the Pt heater structures for the $3 \omega$ measurement is shown in FIG. 3. Subsequently, the $\mathrm{SiO}_{2}$ insulating layer and the $200 \mathrm{~nm}$ thick Pt layer have been deposited by ion beam deposition in a negative resist mask that has been fabricated by electron beam lithography. After deposition of the films, the resist is removed by solving in acetone followed by a lift-off process. To minimize possible electric short circuits between the heater and the film, the size of the heater area on top of the $\mathrm{Sb}_{2} \mathrm{Te}_{3}$ film is minimized. Consequently, the $\mathrm{Sb}_{2} \mathrm{Te}_{3}$ film covers only one corner of the substrate. This allows depositing three heater structures, one on the substrate and two close to the film, where only the relevant transport strip is on top of the thermoelectric film, respectively. This geometry has been chosen for verification. The heater on top of the $\mathrm{SiO}_{2} /$ substrate is used for measuring the thermal conductivity of the $\mathrm{SiO}_{2}$ insulating layer.

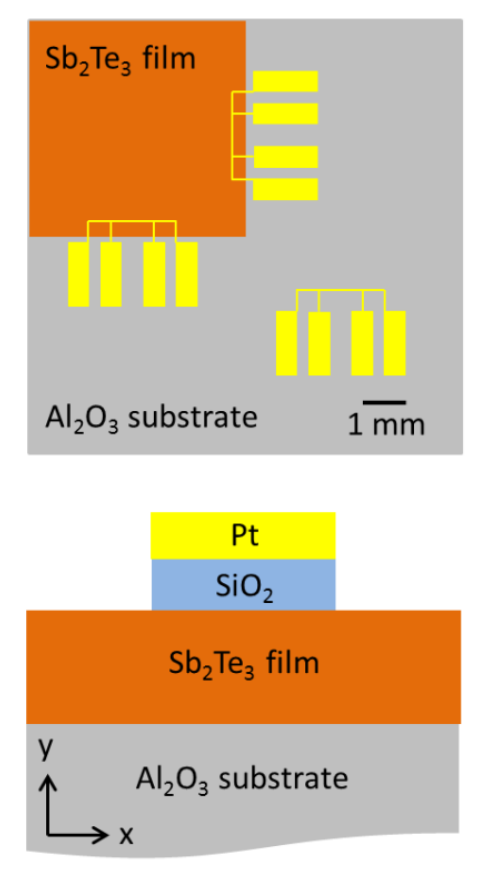

FIG. 3. Top view and cross section of the sample geometry and the used heater structure for the $3 \omega$ measurement. The coordinate system is used in the formula shown below. 
The thicknesses of the film, the $\mathrm{SiO}_{2}$ layer and the Pt heater are measured by profilometry, $\mathrm{X}$ ray reflectometry and TEM. The heater width $2 b$ has been measured via SEM for every individual heater structure. Due to highly reproducible lithography procedures, the variations are with $2 b=10.55 \pm 0.1 \mu \mathrm{m}$ very small. The total heater length is $2 \mathrm{~mm}$; the segment between the voltage sense connections has a length of $1 \mathrm{~mm}$. The TEM characterization of the $\mathrm{SiO}_{2} / \mathrm{Pt}$ stack (FIG. S2 in the Supplementary Information) shows a very flat $\mathrm{SiO}_{2}$ film with complete surface coverage. Selection of the minimum $\mathrm{SiO}_{2}$ thickness of $60 \mathrm{~nm}$ was made based on exclusion of electric shortcuts. Samples have been electrically contacted by silver paste and soldering.

\section{Thermal conductivity measurements by the $3 \omega$ method}

Cross plane thermal conductivity measurements of the films have been performed by the $3 \omega$ technique in the temperature range from 40 to $310 \mathrm{~K}$ in high vacuum. The temperature was controlled by using an electric heater and simultaneously a closed cycle helium cryostat. Used frequencies for the AC current through the heater structure were in the range of 210 to $1010 \mathrm{~Hz}$. The $3 \omega$ set up in Göttingen is based on a description of Raudzis ${ }^{27}$ and further optimized with respect to reference resistances and software control. An eLockIn 204/2 (Anfatec, Germany) is used to extract the $\mathrm{U}_{3 \omega}$ signal.

We analyze the data based on an extension of the method of Cahill ${ }^{28}$, taking into account the anisotropy of film, the heat spread in the film as well as the heat transfer conditions at the backside of the substrate as suggested by Borca-Tasciuc et al. ${ }^{29}$. We found approximately adiabatic boundary conditions at the substrate backside thermal contact. In the following, the indexes 's' and ' $\mathrm{f}$ ' are used for substrate and film, respectively. We fit the $\Delta T(\ln (\omega))$ data to the frequency dependent integral function

$$
\begin{gathered}
|\Delta \mathrm{T}|=\left|\frac{P}{l \pi \kappa_{s, y}} \int_{0}^{\infty} \frac{\sin ^{2}(k b)}{\tanh \left(t_{s} \sqrt{\eta_{s} k^{2}+q^{2}}\right)(k b)^{2} \sqrt{\eta_{s} k^{2}+q^{2}}} d k+\Delta T_{t o t}\right| \\
\kappa_{\mathrm{f}, \mathrm{y}}=\sqrt{\frac{1}{4} \Xi^{2}+\frac{\eta_{s}}{\eta_{f}} \cdot \kappa_{s, y}^{2}}-\frac{1}{2} \Xi \quad \text { (1b) } \\
\Xi=\frac{\eta_{s}}{\eta_{f}} \cdot \frac{2 b l}{t_{f} P} \cdot \Delta T_{f} \cdot \kappa_{s, y}^{2} \quad \text { (1c) }
\end{gathered}
$$

where $\kappa_{s, y}$ and $\kappa_{f, y}$ are the cross plane components of the thermal conductivities, $\Delta T$ is the total thermal offset in the heater, $\Delta T_{\text {tot }}$ is the out of plane component of the offset created by the film, insulation layer and Kapitza resistances, $\Delta T_{f}$ is the offset created by the film only, $P / l$ is the applied power per length of the heater, $2 b$ is the width of the heater, $t_{s}$ and $t_{f}$ are the thicknesses. Furthermore, $\mathrm{q}$ is the inverse of the thermal penetration depth of the substrate, which depends on temperature and frequency. The fitting parameters are $\kappa_{s, y}$ and $\Delta T_{f}$. Using the absolute value of the $\Delta T(\omega)$ oscillation, one can avoid the measurement of phase shifts. This is often a critical 
source of errors in $3 \omega$ measurements. Equations (1b) and (1c) are derived from Tong et al. ${ }^{30}$ as shown in the supplement. Equation (1b) includes a correction for horizontal heat transfer through the film. It is used for more accurate results, even though here it only has a very small impact due to high substrate and low film thermal conductivities. We use anisotropy ratios for substrate and film of $\eta_{s}=0.93$ and $\eta_{f}=2.3$, respectively. The changes in the determined c-axis thermal conductivity of the films in comparison to the isotropic limit $\eta_{s}=\eta_{f}=1$ are however within error bars.

The shown error bars are calculated by error propagation from equations (1), using the experimental errors in the heater width and length, film thickness and the standard deviation of the two fit parameters $\Delta T_{\text {tot }}$ and $\kappa_{s, y}$ in Eq. (1a).

In order to determine the intrinsic thermal conductivity of the thermoelectric $\mathrm{Sb}_{2} \mathrm{Te}_{3}$ films, the subtraction of the thermal conductivity of the $\mathrm{SiO}_{2}$ insulation layer and the Kapitza resistances is necessary. For that reason, a $\mathrm{SiO}_{2}$ layer thickness series for the Kapitza resistances in the reference samples has been prepared and measured containing 9 samples from 10 to $200 \mathrm{~nm}$ $\mathrm{SiO}_{2}$ thicknesses. Furthermore, 7 samples of $\mathrm{Sb}_{2} \mathrm{Te}_{3}$ with thicknesses between 191 and $1184 \mathrm{~nm}$ were prepared and measured, in order to determine the Kapitza resistances of the two interfaces involving the $\mathrm{Sb}_{2} \mathrm{Te}_{3}$ film (see FIG. 4).

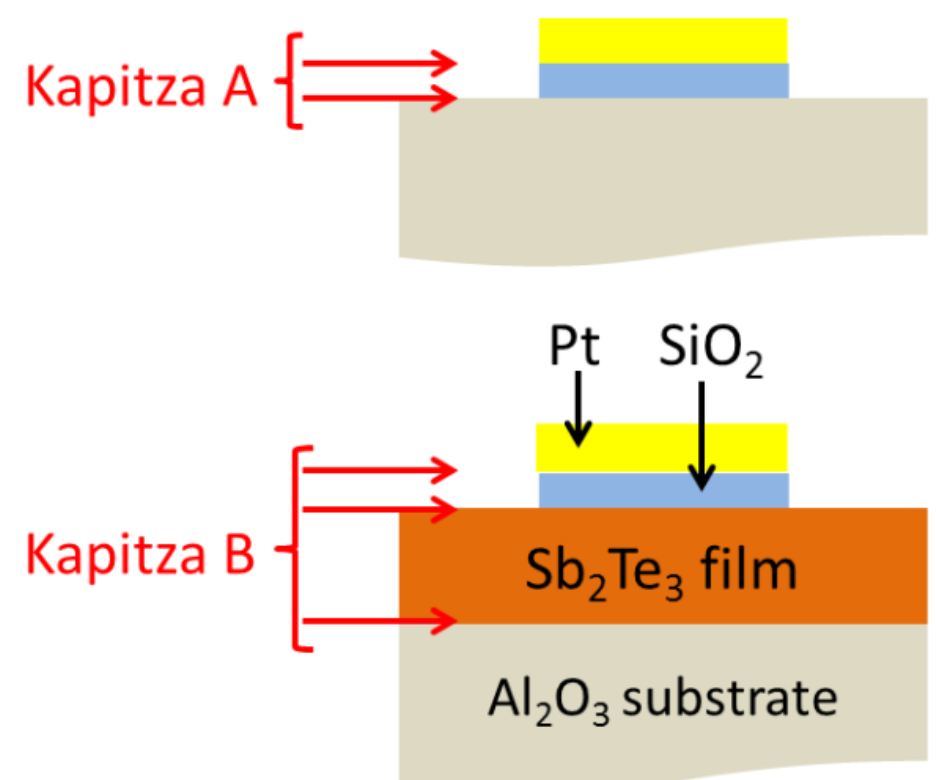

FIG. 4. Sketch of the cross sections of samples of the $\mathrm{SiO}_{2}$ and the $\mathrm{Sb}_{2} \mathrm{Te}_{3}$ thickness series, showing the interfaces that are involved in the Kapitza resistances A and B. 


\section{RESULTS}

\section{A. Determination of the Kapitza resistances}

The Kapitza resistance of a pair of interfaces of a thin film can be determined by studying the $\Delta T$ oscillation as a function of the film thickness $d$ and extrapolating to $\Delta T(d \rightarrow 0)$. In our stack of thin films, two different Kapitza resistances have to be considered: (i) The Kapitza resistance of the two interfaces connected to the $\mathrm{SiO}_{2}$ insulation layer $\left(\mathrm{SiO}_{2}-\mathrm{Sb}_{2} \mathrm{Te}_{3}\right.$ and $\left.\mathrm{SiO}_{2}-\mathrm{Pt}\right)$ which are named Kapitza A in the following. (ii) The Kapitza resistance of the two interfaces connected to the $\mathrm{Sb}_{2} \mathrm{Te}_{3}$ film $\left(\mathrm{Al}_{2} \mathrm{O}_{3}-\mathrm{Sb}_{2} \mathrm{Te}_{3}\right.$ and $\left.\mathrm{Sb}_{2} \mathrm{Te}_{3}-\mathrm{SiO}_{2}\right)$ plus the $\mathrm{SiO}_{2}-\mathrm{Pt}$ interface at the Pt heater, named Kapitza $\mathrm{B}$, in the following.

In order to determine Kapitza $\mathrm{A}, \Delta \mathrm{T}$ was measured as a function of the $\mathrm{SiO}_{2}$ layer thickness $\mathrm{d}_{\mathrm{SiO} 2}$ (Supplemental Information, FIG. S3). Below $60 \mathrm{~nm} \mathrm{SiO} 2$ thickness, a linear dependence of $\Delta \mathrm{T}\left(\mathrm{t}_{\mathrm{SiO} 2}\right)$ is observed which turns into a nonlinear increase at higher $\mathrm{SiO}_{2}$ thickness most probably due to thickness dependent microstructural changes. Both the linear fit between $10 \mathrm{~nm}$ to $60 \mathrm{~nm}$ as well the polynomial $2^{\text {nd }}$ order fit between $10 \mathrm{~nm}$ and $200 \mathrm{~nm}$ give similar results for $\Delta T\left(d_{S_{i O 2} \rightarrow 0}\right)$ (See Supplemental Information FIG. S4). The results for the linear fits as a function of temperature are shown in FIG. 5.

For determination of Kapitza B, $\Delta T$ was measured as a function of the $\mathrm{Sb}_{2} \mathrm{Te}_{3}$ layer thickness $t_{f}$, showing a linear dependence of $\Delta \mathrm{T}\left(\mathrm{t}_{\mathrm{f}}\right)$ over the entire thickness range from $t_{f}=191 \mathrm{~nm}$ to 1184 $\mathrm{nm}$. An exemplary fit is shown in the supplement FIG. S5. The resulting Kapitza B resistance as a function of temperature is also shown in FIG. 5. The $\mathrm{SiO}_{2}$ contribution has already been subtracted. Below $100 \mathrm{~K}$ the thermal penetration depth in the $\mathrm{Al}_{2} \mathrm{O}_{3}$ substrate is too large for the used adiabatic boundary condition for the $3 \omega$ data evaluation. This is due to the strong rise in the thermal conductivity in $\mathrm{Al}_{2} \mathrm{O}_{3}$ at this temperature range, where thermal waves can be reflected at the backside of the substrate. In the measured temperature range Kapitza B is constant. This might be due to the relatively low Debye temperature of $\mathrm{Sb}_{2} \mathrm{Te}_{3}$ of $157 \mathrm{~K}$. Kapitza B is found to be much higher than Kapitza A. This may be explained by three different properties: The Debye temperature in $\mathrm{Al}_{2} \mathrm{O}_{3}$ is with $1047 \mathrm{~K}$ much above that of $\mathrm{Sb}_{2} \mathrm{Te}_{3}$ and thus a huge acoustic mismatch between $\mathrm{Sb}_{2} \mathrm{Te}_{3}$ and $\mathrm{Al}_{2} \mathrm{O}_{3}$ is expected. Additionally, the weak van der Waals bonding between substrate and film might contribute to a huge thermal boundary resistance. Furthermore, $\mathrm{Sb}_{2} \mathrm{Te}_{3}$ shows an electronic contribution to the thermal conductivity while $\mathrm{Al}_{2} \mathrm{O}_{3}$ is an electric insulator. 


\section{B. Determination of the cross plane thermal conductivity of $\mathrm{Sb}_{2} \mathrm{Te}_{3}$}

Since the Kapitza resistances significantly contribute to the total $\Delta T$ oscillation of the film stack, the thermal conductivity of the $\mathrm{Sb}_{2} \mathrm{Te}_{3}$ film would be underestimated. In a first step, the Kapitza A thermal offset has been subtracted from the offset of the reference measurement of the $\mathrm{SiO}_{2}$ insulation layer, in order to get the $\Delta T$ caused by the $\mathrm{SiO}_{2}$ layer only. The resulting contribution of $\mathrm{SiO}_{2}$ and Kapitza B have then been subtracted from the thermal offset of the stack in order to obtain the intrinsic out of plane thermal conductivity of those $\mathrm{Sb}_{2} \mathrm{Te}_{3}$ films.

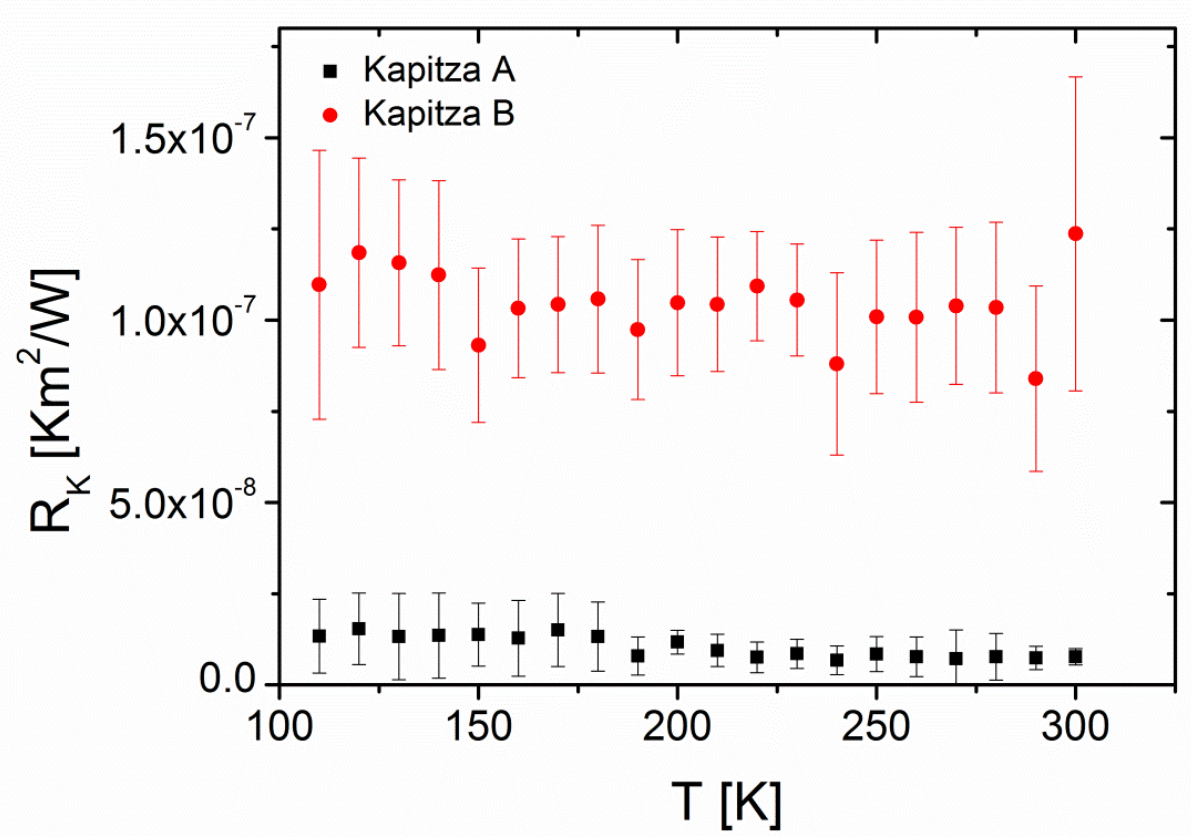

FIG. 5. The Kapitza resistances A and B as defined in FIG. 4 determined from a series of samples with different $\mathrm{SiO}_{2}$ and $\mathrm{Sb}_{2} \mathrm{Te}_{3}$ layer thicknesses as described in the text.

As a result, a precise determination of the intrinsic thermal conductivity as a function of temperature in the range between $100 \mathrm{~K}$ and $300 \mathrm{~K}$ is achieved. The influence of the Kapitza resistances can be seen in FIG. 6. Obviously, the correction of the interface resistances is very important for the determination of the intrinsic $\kappa_{c}(\mathrm{~T})$ of $\mathrm{Sb}_{2} \mathrm{Te}_{3}$. The resulting measurement errors are low for small $\kappa_{\mathrm{c}}$ values due to the corresponding high thermal offset. On average they are estimated to be below $15 \%$ which is quite reasonable for thermal thin film measurements. 


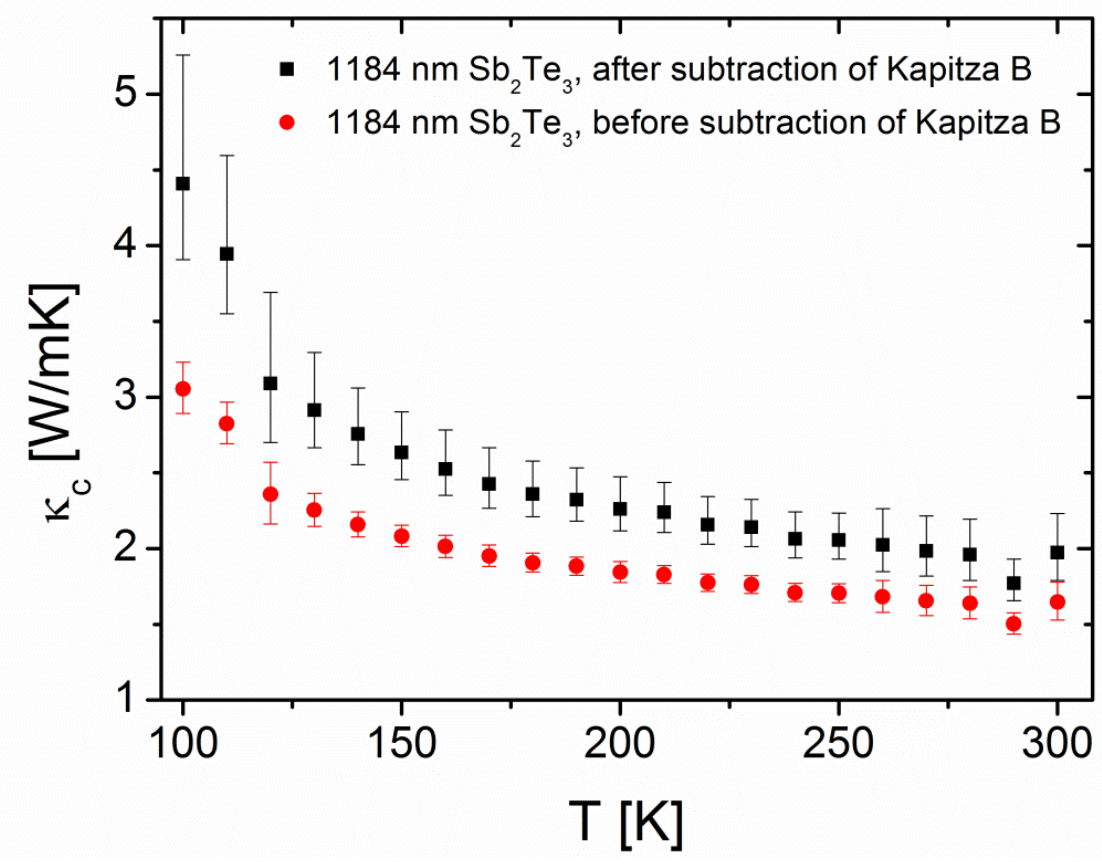

FIG. 6. Thermal conductivity of a representative $\mathrm{Sb}_{2} \mathrm{Te}_{3}$ film without (red) and with (black) subtraction of the Kapitza resistance B.

\section{HRTEM results}

Some samples from the $\mathrm{Sb}_{2} \mathrm{Te}_{3}$ layer thickness series have been investigated using HRTEM and HRSTEM to gain information about their atomic structure and density of defects. Exemplary images are shown for a $191 \mathrm{~nm}$ sample in FIG. 7A and 7B. The study of the film-substrate interface using EELS shows that all samples start growing with one or two quintuple layers of partially oxidized $\mathrm{Sb}_{2} \mathrm{Te}_{3-\mathrm{x}}$ and being also visible in the enhanced disorder of one monolayer in the HRTEM images.

EELS results at three positions marked at FIG. 7B are shown in FIG. 7C. They are based on the analysis of the energy range of 450 to $650 \mathrm{eV}$. We used the $\mathrm{Sb} \mathrm{M}$ edge $(528 \mathrm{eV})$, the $\mathrm{O} \mathrm{K}$ edge $(532 \mathrm{eV})$ and the Te $\mathrm{M}$ edge $(572 \mathrm{eV})$ to obtain the relative concentrations by using Gaussian plots. An exponential background has been subtracted from the original high energy loss spectra. The original data is shown in FIG. S6. The background subtracted spectra together with the used Gaussian fits can be seen in the supplemental FIGs. S7, S8, S9. Since the energy edges of all three elements are close, the overlap of the maxima is the origin of possible errors. Nevertheless, the result fits very well to our expectations: Position 1 on the substrate shows only $\mathrm{O}$ as it is located on the $\mathrm{Al}_{2} \mathrm{O}_{3}$ substrate while Position 3 approximately $6 \mathrm{~nm}$ away from the substrate consists of only $\mathrm{Sb}$ and $\mathrm{Te}$ in the expected ratio of 2:3. Position 2 at the first quintuple layer exhibits a slightly higher $\mathrm{Sb}$ to Te ratio and a small $\mathrm{O}$ content. We suggest the formation of 


\section{CHAPTER 4 : Publication 1}

$\mathrm{Sb}$ enriched $\mathrm{Sb}_{2} \mathrm{Te}_{3-\mathrm{x}} \mathrm{O}_{\mathrm{y}}$ during film growth by intermixing with surface oxygen from the substrate. This interfacial layer exhibits significant disorder visible in the high resolution images, however its presence does not affect epitaxial growth of the $\mathrm{Sb}_{2} \mathrm{Te}_{3}$.
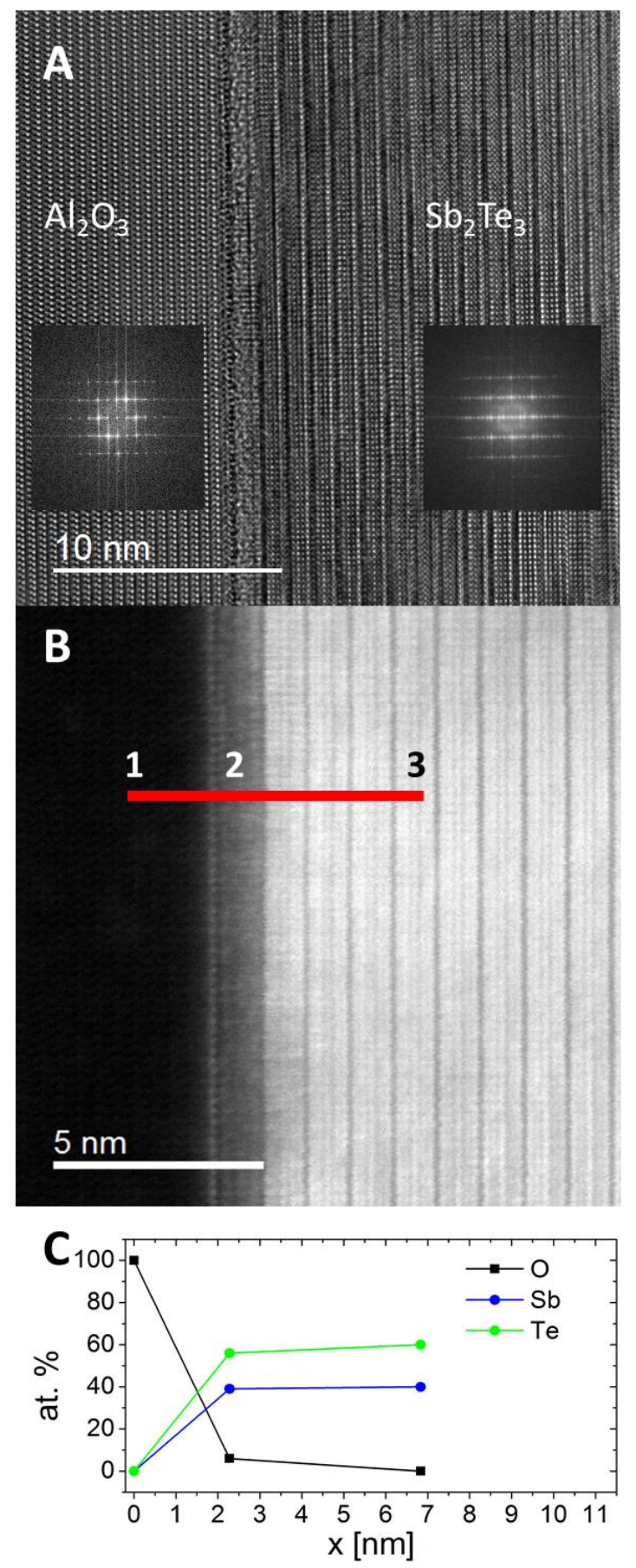

FIG. 7. Interface $\mathrm{Al}_{2} \mathrm{O}_{3}-\mathrm{Sb}_{2} \mathrm{Te}_{3}$ (191 nm thick). (A) HRTEM image and, FFTs show epitaxial c-axis oriented film. (B) STEM image of the interface, (C) EELS results for O K edge, Sb M edge and Te $\mathrm{M}$ edge at three different positions marked in $\mathrm{B}$. 
A very low defect concentration can be observed in all samples. The concentration of stacking faults is increasing slowly starting at approximately $150 \mathrm{~nm}$ thickness with the increasing distance to the substrate. Moreover, the $1184 \mathrm{~nm}$ thick $\mathrm{Sb}_{2} \mathrm{Te}_{3}$ film shows the formation of differently oriented surface grains. They may be formed due to a reduced growth temperature at increasing film thickness due to the thermal gradient between substrate and the top area of the film related to the low thermal conductivity of $\mathrm{Sb}_{2} \mathrm{Te}_{3}$.

\section{DISCUSSION}

After careful subtraction of the extrinsic contributions of the interfaces and the insulation layers, the determined intrinsic out of plane thermal conductivity $\kappa_{c}(T)$ of $\mathrm{Sb}_{2} \mathrm{Te}_{3}$ epitaxial films of different thicknesses are shown in FIG. 9A. Deviations for T $<140 \mathrm{~K}$ are because of $q^{-1} \rightarrow t_{s}$ and thus the used adiabatic thermal boundary condition for the back contact of the substrate is getting increasingly inaccurate with decreasing $\mathrm{T}$. C-axis conductivity of a representative film is compared to $\kappa_{c}$ and $\kappa_{a, b}(T)$ data from literature in FIG. 9B. We have selected the thickest film because of lowest error. In literature, we have only found c-axis thermal conductivity data for $\mathrm{Sb}_{2} \mathrm{Te}_{3}$ single crystals at room temperature ${ }^{26}$. It was determined by measuring the thermal diffusivity via an open-cell photoacoustic method in the rear-side illumination configuration. The measurement was performed on $\mathrm{Sb}_{2} \mathrm{Te}_{3}$ single crystals prepared in sealed quartz ampullas at $850{ }^{\circ} \mathrm{C}$. To the best of our knowledge, no temperature dependent $\kappa_{c}(T)$ values are available in literature. The value $\kappa_{\mathrm{c}}=1.65 \pm 0.11 \mathrm{~W} / \mathrm{mK}$ given by Yáñez-Limón et al. ${ }^{26}$ well agrees with our $\kappa_{c}(300 K)$ data for epitaxial thin films. This confirms the low defect concentration and high crystallinity of our PVD grown c-oriented $\mathrm{Sb}_{2} \mathrm{Te}_{3}$ films as found by HRTEM analysis. 


\section{CHAPTER 4 : Publication 1}

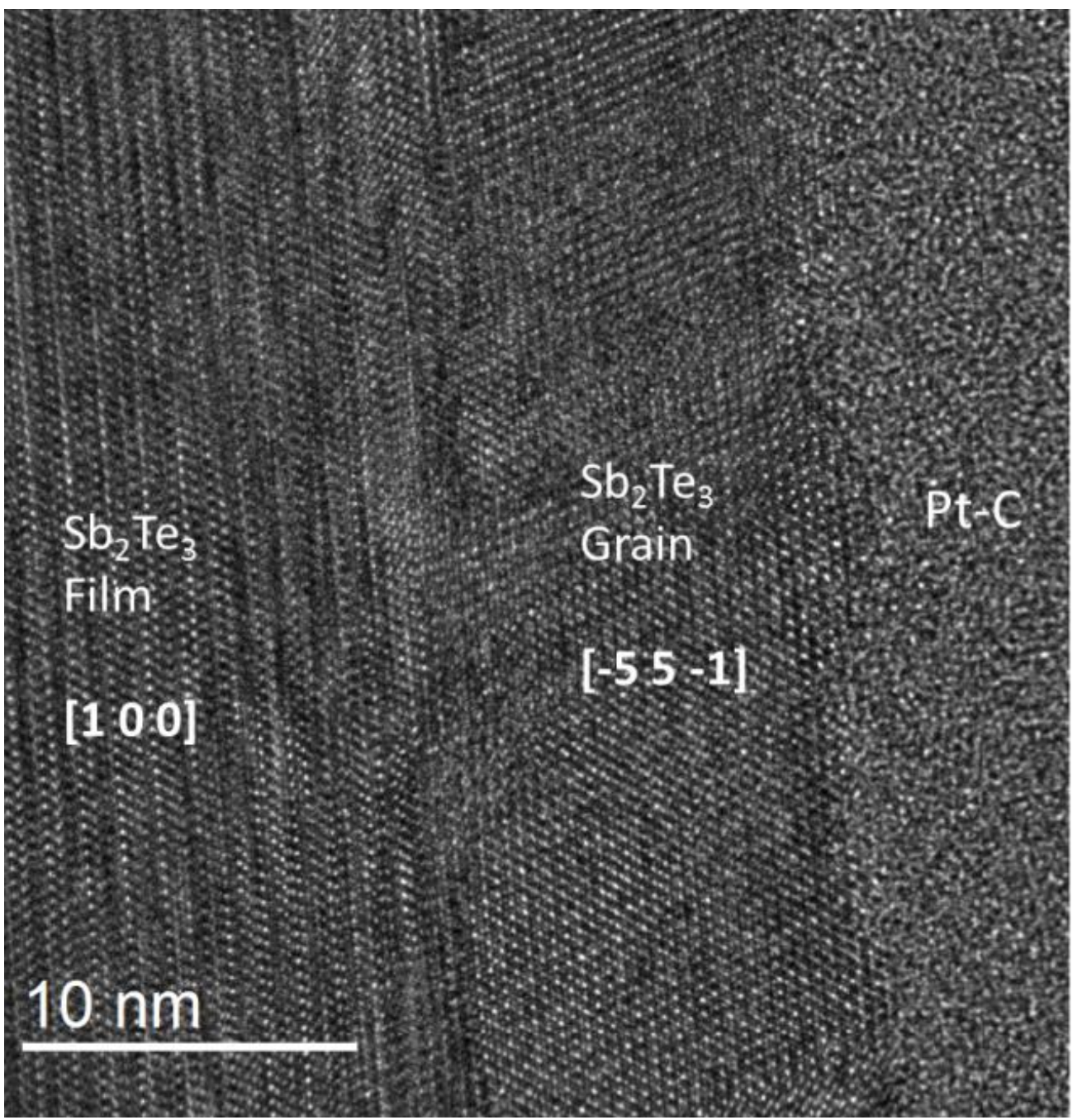

FIG. 8. Upper part of a $1184 \mathrm{~nm}$ thick $\mathrm{Sb}_{2} \mathrm{Te}_{3}$ film covered by the Pt-C protection layer. It shows a slightly higher defect concentration followed by thin grains with different orientation but same composition. 

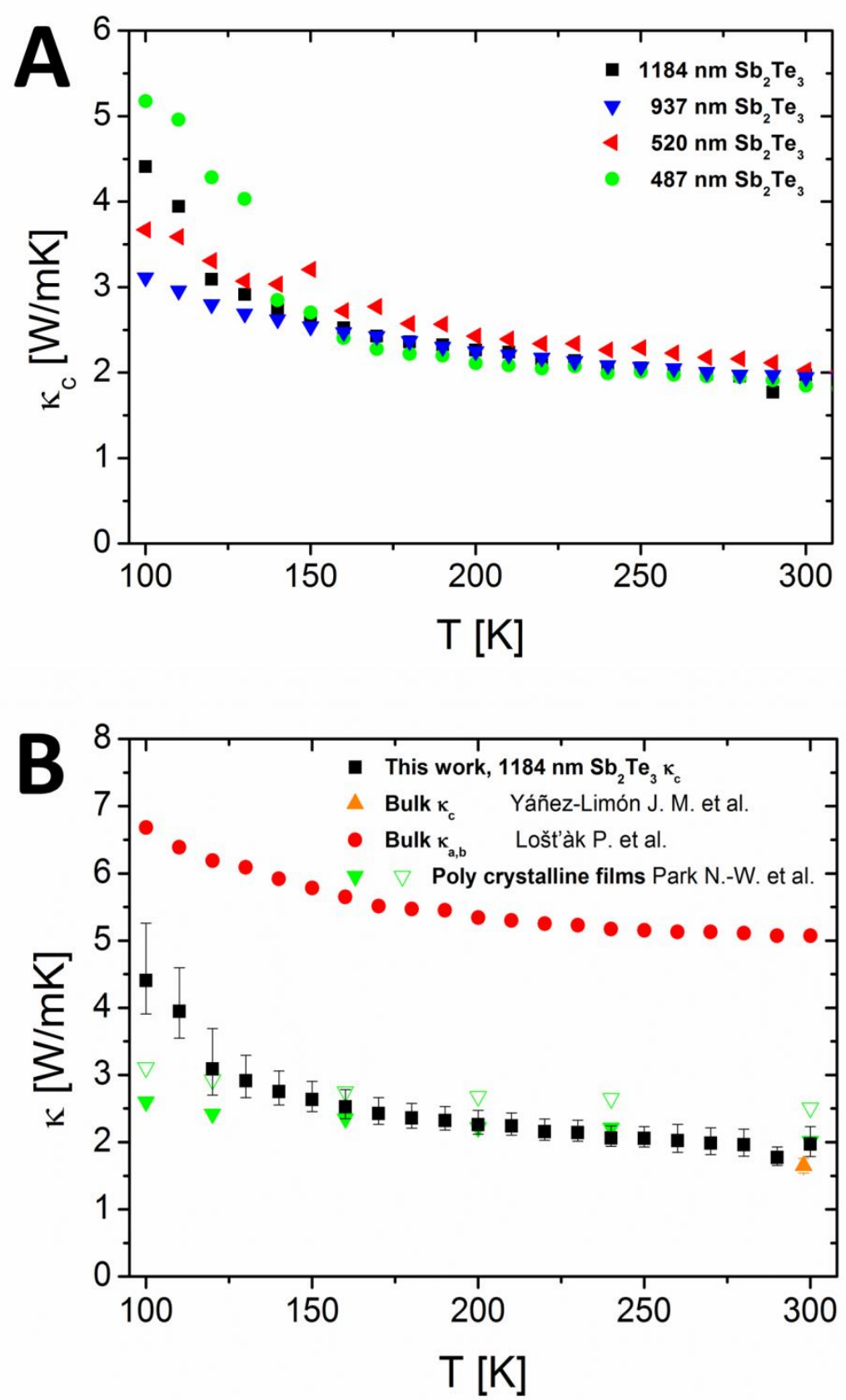

FIG. 9. (A) C-axis thermal conductivity $\kappa_{c}$ of the PVD grown $\mathrm{Sb}_{2} \mathrm{Te}_{3}$ films of different thicknesses after correction for Kapitza resistances and the contribution of the $\mathrm{SiO}_{2}$ insulation layer. (B) The result for $\kappa_{c}(T)$ for the $1184 \mathrm{~nm}$ thick film is compared with the room temperature value of $\kappa_{c}$ and $\kappa_{a, b}(T)$ measured at single crystalline bulk $\mathrm{Sb}_{2} \mathrm{Te}_{3}{ }^{26,24}$. In addition, the thermal conductivity of nanocrystalline sputtered $\mathrm{Sb}_{2} \mathrm{Te}_{3}$ films with two different average grain sizes $88 \mathrm{~nm}$ (filled green triangles) $129 \mathrm{~nm}$ (empty green triangles) is presented for comparison ${ }^{1}$. 
The in-plane thermal conductivity $\kappa_{a b}(T)$ was measured by a longitudinal steady-state technique at $\mathrm{Sb}_{2} \mathrm{Te}_{3}$ single crystals grown by a modified Bridgeman method ${ }^{24}$. The $\kappa_{a b}(T)$ is several times larger than the measured $\kappa_{c}(T)$ of our epitaxial films, indicating temperature dependent anisotropy $A(T)$ between 1.5 and 2.6 (ignoring the obviously deviating value of our measurement at $290 \mathrm{~K}$ ).

FIG. 9B also shows the thermal conductivity of nanocrystalline films prepared by RF-magnetron sputtering at room temperature on $\mathrm{Si}$ measured by the $3 \omega$ method $^{1}$. The films have been annealed at different temperatures between $200{ }^{\circ} \mathrm{C}$ and $350{ }^{\circ} \mathrm{C}$ in order to modify the grain size between $88 \mathrm{~nm}$ and $129 \mathrm{~nm}$. Without the effect of grain boundary scattering, the average thermal conductivity in nanocrystalline films would be given by $\kappa_{a v}=\sqrt{\kappa_{c} \kappa_{a b}}=\kappa_{c} \sqrt{A} \approx$ (1.2-1.6) $\kappa_{c}$. Indeed, as shown in FIG. 9B, above $\mathrm{T}=200 \mathrm{~K}$, the thermal conductivity of the nanocrystalline films is close to the $\kappa_{c}(T)$ of our single crystalline films. Consequently, the effect of grain boundary scattering at grain sizes of the order of $100 \mathrm{~nm}$ seems to be quite low and only visible at temperatures below $120 \mathrm{~K}$.

Next, we apply a simple approximation of kinetic theory for the phonon thermal conductivity to correlate the estimated phonon mean free path $l_{m f p}$ with the average defect density in our epitaxial films. In order to get an estimate of $l_{m f p}$, the electronic contribution to $\kappa_{c}$ must be subtracted, i.e.

$$
\kappa_{c, l}(T)=\kappa_{c}-\kappa_{c, e} \approx \frac{1}{3} C_{V} v l_{m f p},
$$

where $\kappa_{\mathrm{c}, \mathrm{e}}$ is the electron contribution, $C_{v}$ is the specific heat and $\mathrm{v}$ is the average velocity of acoustic phonon modes. We take $\kappa_{c, e}=1.03 \mathrm{~W} / \mathrm{mK}$ from ref. ${ }^{26} . C_{v}(T)$ from 4 up to $280 \mathrm{~K}$ has been published by Dutta et al. ${ }^{31}$. We determine $C_{v}(300 \mathrm{~K})$ by extrapolation since for $\mathrm{Sb}_{2} \mathrm{Te}_{3}$ the increase is very small in that temperature region. The sound velocity parallel to the $c$ axis $v_{c}$ has been obtained using the stiffness matrix $\mathrm{C}$ (ref. ${ }^{32}$ ) and the density $\rho$ by using the following formula adopted from Zheng et al. ${ }^{33}$ :

$$
v_{c}=\frac{1}{3} v_{L}+\frac{2}{3} v_{T}=\frac{1}{3} \sqrt{\left(\frac{C_{33}}{\rho}\right)}+\frac{2}{3} \sqrt{\left(\frac{C_{44}}{\rho}\right)}
$$

Temperature dependent measurement of $\sigma_{c}$ published by Lošt'ák et al. ${ }^{25}$ reveals an almost constant $\kappa_{c, e}(T)$. We take a temperature independent absolute value of $\kappa_{\mathrm{c}, \mathrm{e}}$ from ref. ${ }^{26}$. The result for the phonon mean free path $1_{\mathrm{mfp}}$ parallel to the c-axis is shown in FIG. 10: The order of magnitude of $1_{\mathrm{mfp}}$ is in the range of only a few $\mathrm{nm}$ for all temperatures and shows a monotonously decreasing temperature dependence. 


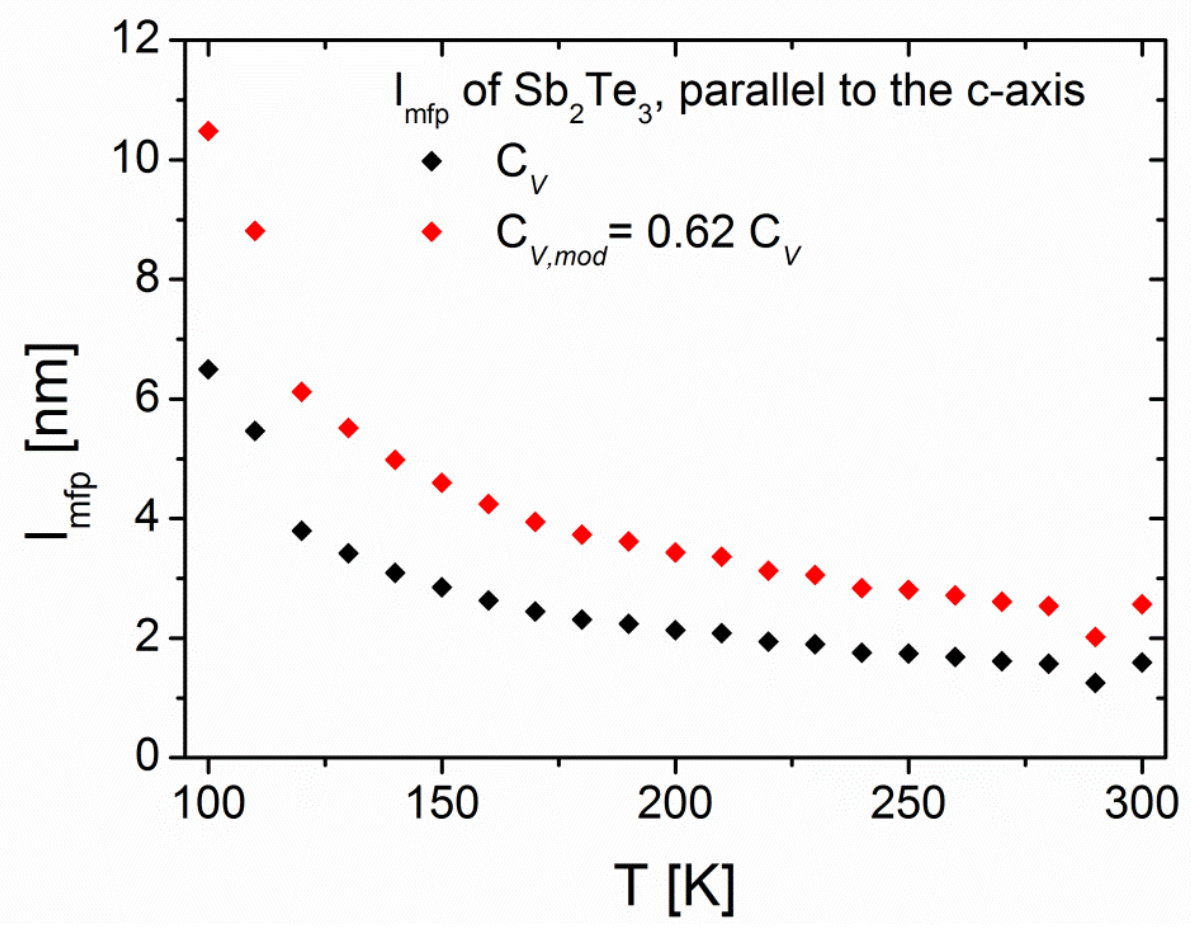

FIG. 10. Phonon mean free path of $\mathrm{Sb}_{2} \mathrm{Te}_{3}$ parallel to the c-axis, based on $\kappa_{\mathrm{c}}$ calculated as described in the text.

The approximation of Eq. (2) for an estimate of the mean free path of a representative phonon from the lattice thermal conductivity is only valid in isotropic materials. For strongly anisotropic $\mathrm{vdW}$ materials such as graphite $(A=300)$, this equation has led to a strong underestimate of $l_{m f p}$ in c-direction, because of a strong variation of the density of states in different directions ${ }^{34,35,36}$. This has the consequence that specific heat transported into the c-axis is reduced compared to the isotropic value $\frac{1}{3} C_{V}(T)$. In comparison to graphite, the thermal conductivity anisotropy of $\mathrm{Sb}_{2} \mathrm{Te}_{3}$ is however two orders of magnitude smaller. We thus suggest correcting to the specific heat transported in c-direction by the anisotropy ratio of the highest acoustic phonon frequency in the c- and ab-directions, respectively:

$$
C_{V, \bmod }=C_{V} \frac{\omega_{a c, c}}{\omega_{a c, a b}}(4)
$$

Using $\omega_{a c, c} / \omega_{a c, a b} \approx 0.62$ from ref. ${ }^{37}$, the estimate of $l_{m f p}$ in c-direction is increased by around $60 \%$ as shown in FIG. 10.

In comparison, the observed density of the stacking faults in the $487 \mathrm{~nm}$ thick $\mathrm{Sb}_{2} \mathrm{Te}_{3}$ film is approx. $10^{10} / \mathrm{cm}^{2}$, in the sample with $1184 \mathrm{~nm}$ thickness it is estimated to be around $2 \cdot 10^{10} / \mathrm{cm}^{2}$. 
This translates into a mean distance between stacking faults parallel to the c-axis of about 50 to $100 \mathrm{~nm}$ and is thus much larger than the estimated phonon mean free path. Although we cannot directly measure the density of point defects, we argue that their impact on $1_{\text {mfp }}$ in PVD grown films is negligible. Since the growth of PVD films proceeds near to thermal equilibrium, the point defect concentration should be close to the thermal equilibrium concentration. For example, the defect of lowest formation energy $\Delta E$ in $\mathrm{Sb}_{2} \mathrm{Te}_{3}$ is the antisite defect with $\Delta E=0.35$ $\mathrm{eV}$ and a measured room temperature concentration of $8-10 \times 10^{19} \mathrm{~cm}^{-3}$ (see ref. ${ }^{38}$ ). This translates in an anti-site defect in 1 out of 26 unit cells and thus an average distance in cdirection of $81.4 \mathrm{~nm}$.

Note that $l_{m f p}$ in c-direction derived from the kinetic theory may represent the lower bound values, even after correction for anisotropy. Taking the phonon dispersion into account typically yields a higher $l_{m f p}$ as e.g. shown for silicon ${ }^{39}$. In addition, a recent study ${ }^{40}$ has shown that for a $\mathrm{vdW}$ material $\left(\mathrm{Ta}_{2} \mathrm{Pd}_{3} \mathrm{Se}_{8}\right)$ of less degree of anisotropy than graphite, the $l_{m f p}$ along the vdW interaction direction could be tens of nanometers long. Our conclusion about the intrinsic origin of the low $\kappa_{c}(T)$ in our $\mathrm{Sb}_{2} \mathrm{Te}_{3}$ films thus depends on the assumption that phonon dispersion affects $l_{m f p}$ as obtained by kinetic theory by less than one order of magnitude.

\section{SUMMARY AND CONCLUSION}

We have carried out careful measurements of the c-axis thermal conductivity in high quality nearly single crystalline epitaxial $\mathrm{Sb}_{2} \mathrm{Te}_{3}$ thin films using the $3 \omega$ method. In order to determine the intrinsic $\kappa_{\mathrm{c}}(T)$, the contribution of the Kapitza resistances and the thermal resistance of the electrical insulation layer is measured. The obtained Kapitza resistance of the two interfaces between the layered $\mathrm{Sb}_{2} \mathrm{Te}_{3}$ film sandwiched by $\mathrm{Al}_{2} \mathrm{O}_{3}$ and $\mathrm{SiO}_{2}$ is with $\mathrm{R}_{\mathrm{K}} \geq 10^{-7} \mathrm{Km}^{2} / \mathrm{W}$ relatively large. However, the value fits well to the range of values of interfaces with large acoustic impedance mismatch ${ }^{41}$. Our result on the intrinsic c-axis thermal conductivity of epitaxial thin films indeed confirms the low $\kappa_{\mathrm{c}}(300 \mathrm{~K})$ value of an $\mathrm{Sb}_{2} \mathrm{Te}_{3}$ single crystal in ref. ${ }^{26}$. After taking into account the electronic contribution to $\kappa_{\mathrm{c}}(\mathrm{T})$, we approximately calculate the temperature dependent phonon mean free path. We argue that the intrinsic phonon mean free path due to phonon-phonon scattering is dominating $\kappa_{\mathrm{c}}(\mathrm{T})$ above the Debye temperature $\left(\theta_{\mathrm{D}}=157 \mathrm{~K}^{42}\right)$. Although we cannot entirely rule out the effect of point defects on $1_{\mathrm{mfp}}$, their effect at thermal equilibrium concentration is small. Consequently, we obtain a low intrinsic $\kappa_{c}(T)$ in epitaxial highly crystalline $\mathrm{Sb}_{2} \mathrm{Te}_{3}$ films grown by PVD close to thermal equilibrium.

We observe a weak decrease of $\kappa_{c}(T)$ with increasing temperature. Such a temperature characteristic is typically observed for the c-direction in van de Waals bound layered systems with relatively low Debye temperature such as $\mathrm{WSe}_{2}$ at low defect density ${ }^{43}$ or $\mathrm{Ca}_{3} \mathrm{Co}_{4} \mathrm{O}_{9}{ }^{44}$ due to phonon-phonon scattering. The low intrinsic $\kappa_{c}(T)$ as well as the temperature characteristics in $\mathrm{Sb}_{2} \mathrm{Te}_{3}$ support the idea of a generally low $\kappa_{c}(T)$ for layered materials with van der Waals 
bonding. Furthermore, we conclude that the reduced thermal conductivity of nanocrystalline films in ref. ${ }^{1}$ compared to $\kappa_{a b}$ is mostly due to the low c-axis contribution, suggesting that an effective pathway for the reduction of the thermal conductivity in c-axis oriented films is predominantly enhancing point defect scattering by alloying of pure systems or by interdiffusion of different chemical species in superlattices.

\section{SUPPLEMENTARY MATERIAL}

In the supplemental document the derivation of equations (1b) and (1c) from literature and all figures named S1 to S9 can be found.

\section{ACKNOWLEDGMENTS}

Financial support by the Deutsche Forschungsgemeinschaft DFG (S. Schulz, project SCHU 1069/20-1; C. Jooss, project JO 348/12-1) is gratefully acknowledged. 


\section{REFERENCES}

\footnotetext{
${ }^{1}$ N.-W. Park, W.-Y. Lee, J.-E. Hong, T.-H. Park, S.-G. Yoon, H. Im, H. S. Kim, and S.-K. Lee, Nanoscale Res. Lett. 10, 20 (2015).

2 T. M. Tritt, and M. A. Subramanian, MRS Bulletin 31, 188 (2006).

${ }^{3}$ D. M. Rowe, Handbook of Thermoelectrics, CRC Press, Boca Raton, 1995.

${ }^{4}$ M. Winkler, X. Liu, A.-L. Hansen, J. D. König, W. Bensch, L. Kienle, H. Böttner, and K. Bartholomé, Nanothermoelectrics 1, 1 (2013).

${ }^{5}$ R. Venkatasubramanian, T. Colpitts, E. Watko, M. Lamvik, and N. El-Masry, J. Cryst. Growth 170, 817 (1997).

${ }^{6}$ R. Venkatasubramanian, E. Siivola, T. Colpitts, and B. O'Quinn, Nature 413, 597 (2001).

${ }^{7}$ A.-L. Hansen, T. Dankwort, M. Winkler, J. Ditto, D. C. Johnson, J. D. Koenig, K. Bartholomé, L. Kienle, and W. Bensch., Chem. Mater. 26, 6518 (2014).

${ }^{8}$ M. Winkler, X. Liu, U. Schürmann, J. D. König, L. Kienle, W. Bensch, and H. Böttner, Z. Anorg. Allg. Chem. 638, 2441 (2012).

${ }^{9}$ For a recent review article see: T. Dankwort, A.-L. Hansen, M. Winkler, U. Schürmann, J. D. Koenig, D. C. Johnson, N. F. Hinsche, P. Zahn, I. Mertig, W. Bensch, and L. Kienle., Phys. Status Solidi A 213, 662 (2016).

${ }^{10}$ M. Eschbach, E. Młyńczak, J. Kellner, J. Kampmeier, M. Lanius, E. Neumann, C. Weyrich, M. Gehlmann, P. Gospodarič, S. Döring, G. Mussler, N. Demarina, M. Luysberg, G. Bihlmayer, T. Schäpers, L. Plucinski, S. Blügel, M. Morgenstern, C. M. Schneider, and D. Grützmacher, Nat. Commun. 6, 8816 (2015).

${ }^{11}$ Y. Jiang, Y. Y. Sun, M. Chen, Y. Wang, Z. Li, C. Song, K. He, L. Wang, X. Chen, Q.-K. Xue, X. Ma, and S. B. Zhang, Phys. Rev. Lett. 108, 066809 (2012).

${ }^{12}$ X. Zhang, Z. Zeng, C. Shen, Z. Zhang, Z. Wang, C. Lin, and Z. Hu, J. Appl. Phys. 115, 024307 (2014).

${ }^{13}$ Y. Saito et al., physica status solidi b, 252, 2151 (2015).

${ }^{14}$ I. Hilmi, A. Lotnyk, J. W. Gerlach, P. Schumacher, and B. Rauschenbach, PL Materials 5, 050701 (2017).

${ }^{15}$ B. Saha, P. Chaturvedi, A. K. Yadav, D. Saha, and S. Ganguly, J. Vac. Sci. Technol. B 34, 021806 (2016).

${ }^{16}$ G. Bendt, S. Zastrow, K. Nielsch, P. S. Mandal, J. Sánchez-Barriga, O. Rader, and S. Schulz, J. Mater. Chem. A 2, 8215 (2014).

17 P. I. Kuznetsov, B. S. Shchamkhalova, V. O. Yapaskurt, V. D. Shcherbakov, V. A. Luzanov, G. G. Yakushcheva, V. A. Jitov, and V. E.Sizov, J. Cryst. Growth 471, 1 (2017).

${ }^{18}$ L. M. Goncalves,, P. Alpuim, A. G. Rolo, and J. H. Correia, Thin Sol. Films 519, 4152 (2011).

${ }^{19}$ H. Shen, S. Lee, J.-g. Kang, T.-Y.Eom, H. Lee, and S. Han, Appl. Surf. Sci. 429, 115 (2018).

${ }^{20}$ J.-H. Kim, J.-Y. Choi, J.-M. Bae, M.-Y. Kim, and T.-S. Oh, Mater. Transactions 54, 618 (2013).

${ }^{21}$ J.-M. Lin, Y.-C. Chen, and W. Chen, J. Nanomaterials 2015, article ID 564954 (2015).
} 
${ }^{22}$ For a recent review article see: Y. Guo, Z. Liu, and H. Peng, Small 11, 3290 (2015).

${ }^{23}$ H. Chi, W. Liu, and C. Uher, Growth and transport properties of tetradymite thin films, in Materials Aspect of Thermoelectricity, $1^{\text {st }}$ ed., Ed. C. Uher, CRC Press, Boca Raton, 2016.

${ }^{24}$ P. Lošt'ák, Č. Drašar, A. Krejčová, L. Beneš, J. S. Dyck, W. Chen, and C. Uher, J. Cryst. Growth 222, 565 (2001).

${ }^{25}$ P. Lošt'ák, Č. Drašar, J. Horàk, Z. Zhou, J. S. Dyck, and C. Uher, J. Phys. Chem. Sol. 67, 1457 (2006).

${ }^{26}$ J. M. Yáñez-Limón, J. González-Hernández, J. J. Alvaro-Gil, and I. Delgadillo, and H. Vargas, Phys. Rev. B 52, 16321 (1995).

${ }^{27}$ C. E. Raudzis (Doctoral Thesis), Der andere Verlag (2007).

${ }^{28}$ D. G. Cahill, Rev. Sci. Instrum. 61, 802 (1990).

${ }^{29}$ T. Borca-Tasciuc, A. R. Kumar, G. Chen, Rev. Sc. Instrum. 72, 2139 (2001).

${ }^{30}$ T. Tong and A. Majumdar, Rev. Sci. Instrum. 77, 104902 (2006).

${ }^{31}$ P. Dutta, D. Bhoi, A. Midya, N. Khan, P. Mandal, S. Shanmukharao Samatham, and V. Ganesan, Appl. Phy. Let. 106, 251912 (2012).

${ }^{32}$ M. de Jong, W. Chen, T. Angsten, A. Jain, R. Notestine, A. Gamst, M. Sluiter, C. Krishna Ande, S. van der Zwaag, J. J. Plata, C. Toher, S. Curtarolo, G. Ceder, K. A. Persson, and M. Asta, Scientific Data 2, 150009 (2015).

${ }^{33}$ D. Zheng, S. Tanaka, K. Miyazaki, and M. Takashiri, J. Electron. Mater. 44, 1679 (2015).

${ }^{34}$ Z. Wei, J. Yang, W. Chen, K. Bi, D. Li, and Yunfei Chen, Appl. Phys. Lett. 104, 081903 (2014).

${ }^{35}$ Q. Fu, J. Yang, Y. Chen, D. Li, and D. Xu, Appl. Phys. Lett. 106, 031905 (2015).

${ }^{36}$ H. Zhang, X. Chen, Y.-D. Jho, and A. J. Minnich, Nano Lett. 16, 1643-1649 (2016).

${ }^{37}$ B.-T. Wang, P. Souvatzis, O. Eriksson, and P. Zhang, The Journal of Chemical Physics 142, $174702(2015)$.

38 J. Horák, K. Čermák, and L. Koudelka, J. Phys. Chem. Solids 47, 805 (1986).

${ }^{39}$ Y. S. Ju and K. E. Goodson, Appl. Phys. Lett. 74, 3005 (1999).

${ }^{40}$ Q. Zhang, C. Liu, X. Liu, J. Liu, Z. Cui, Y. Zhang, L. Yang, Y. Zhao, T. T. Xu, Y. Chen, J. Wei, Z. Mao, and D. Li, ACS Nano 12, 2634 (2018).

${ }^{41}$ H.-K. Lyeo, and D. G. Cahill, Phys. Rev. B 73, 144301 (2006).

${ }^{42}$ A. S. Pashinkin, A. S. Malkova, and M. S. Mikhailova, Russian J. Phys. Chem. 82, 878 (2008).

${ }^{43}$ C. Chiritescu, D. G. Cahill, N. Nguyen, D. Johnson, A. Bodapati, P. Keblinski, and P. Zschack, Science 315, 351 (2007).

${ }^{44}$ L. Wu, Q. Meng, C. Jooss, J.-C. Zheng, H. Inada, D. Su, Q. Li, and Y. Zhu, Adv. Funct. Mater. 23, 5728, (2013). 



\title{
Supplemental information to: Low intrinsic c-axis thermal conductivity in PVD grown epitaxial $\mathrm{Sb}_{2} \mathrm{Te}_{3}$ films
}

\author{
F. Rieger, ${ }^{1}$ K. Kaiser, ${ }^{2}$ G. Bendt, ${ }^{2}$ V. Roddatis, ${ }^{1}$ P. Thiessen, ${ }^{1}$ \\ S. Schulz, ${ }^{2}$ and C. Jooss ${ }^{1}$ \\ ${ }^{1}$ Institute of Material Physics, University of Göttingen, 37077 Göttingen, Germany \\ ${ }^{2}$ Institute of Inorganic Chemistry and Center for Nanointegration Duisburg-Essen \\ (Cenide), University of Duisburg - Essen, 45141 Essen, Germany
}

\section{Derivation of the formula for calculating $\kappa_{f, y}$ from Tong et al.}

Tong et al. ${ }^{1}$ presented a modified data analysis method as presented in formula (s1) ( 2 in their work) in order to evaluate $3 \omega$ results in film-substrate configurations. They modified the formula introduced by Cahill et al. ${ }^{2}$ by a correction term $\Gamma$ and the film-substrate interface resistance $\mathrm{R}_{\mathrm{f}, \mathrm{s}}$. The correction term takes into account basically two different effects, namely the thermal conductivity contrast of film and substrate, and the heat spread in the film, both under consideration of the influence of anisotropy. They derived a dependence of the modification factor of the three parameters $\mathrm{K}, \mathrm{Bi}_{\mathrm{f}}$ and $\mathrm{H}_{\mathrm{r}}$ as shown in equations (s1) and (s2). Note that we changed the designation of some quantities, in order to be consistent to the formulas in the main text.

$$
\begin{gathered}
\Delta T_{t o t}=\frac{P}{l 2 b}\left[\frac{t_{f}}{\kappa_{f, y}} \Gamma\left(\mathrm{K}_{,} \mathrm{Bi}_{\mathrm{f}}, \mathrm{Hr}\right)+\mathrm{R}_{\mathrm{f}, \mathrm{s}}\right] \\
K=\frac{\kappa_{f, y}}{\kappa_{s, y}} \quad B i_{f}=\frac{t_{f}}{\kappa_{f, y} R_{K}} \quad H r=\frac{b}{t_{f} \sqrt{\eta_{f}}}
\end{gathered}
$$

In this section we present the derivation of Equations (1b) and (1c) in the main text from the published results of Tong et al. .

Starting with Equation (s1), we add an additional term for the contribution of the insulation layer $\mathrm{R}_{\mathrm{SiO} 2}$ to the film-substrate interface resistance $\mathrm{R}_{\mathrm{f}, \mathrm{s}}$. We also add the interface thermal resistances between heater and insulation layer and between insulation layer and $\mathrm{Sb}_{2} \mathrm{Te}_{3}$ film. All interface thermal resistances are summed up as Kapitza resistances $R_{K}$, called Kapitza $B$ in the main text. $\mathrm{R}_{\mathrm{K}}$ and $\mathrm{R}_{\mathrm{SiO} 2}$ are experimentally determined by the $\mathrm{SiO}_{2}$ - and $\mathrm{Sb}_{2} \mathrm{Te}_{3}$ - thickness series mentioned in the main text. This leads to Equation (s3). For the calculation of the thermal offset $\Delta \mathrm{T}_{\mathrm{f}}$ caused 
only by the $\mathrm{Sb}_{2} \mathrm{Te}_{3}$ film (Equation (s4)), the sum of the thermal offset contributions of thermal boundary resistance and insulation layer $\Delta T_{K, I}=\frac{P}{l 2 b}\left(R_{K}+R_{S i O_{2}}\right)$ is subtracted from $\Delta \mathrm{T}_{\text {tot. }}$.

$$
\begin{aligned}
& \Delta T_{t o t}=\frac{P}{l 2 b}\left[\frac{t_{f}}{\kappa_{f, y}} \Gamma\left(\mathrm{K}, \mathrm{Bi}_{\mathrm{f}}, \mathrm{Hr}\right)+\mathrm{R}_{\mathrm{K}}+\mathrm{R}_{S^{\prime O} O_{2}}\right] \\
& \Delta \mathrm{T}_{\mathrm{f}}=\Delta T_{t o t}-\Delta T_{K, I}=\frac{P}{l 2 b} \frac{t_{f}}{\kappa_{f, y}} \Gamma\left(\mathrm{K}, \mathrm{Bi}_{\mathrm{f}}, \mathrm{Hr}\right)
\end{aligned}
$$

In principle, Eq. (s4) can be used to derive $\kappa_{\mathrm{f}, \mathrm{y}}$. However, further approximations for the function $\Gamma\left(\mathrm{K}, \mathrm{Bi}_{\mathrm{f}}, \mathrm{Hr}\right)$ are useful. In the range of thermal penetration depths smaller than the substrates thickness (excluding the effect of back reflection of thermal waves at the substrate backside) and much larger than the film thickness $t_{f}$, Tong et al. derived the following equation for the thermal impedance $\Delta \mathrm{Z}$ of film and Kapitza resistances in Fourier space:

$$
\Delta \mathrm{Z}=\frac{\mathrm{t}_{\mathrm{f}}}{\kappa_{\mathrm{f}, y}}\left(1-\frac{\kappa_{f, y}^{2} \eta_{f}}{\kappa_{s, y}^{2} \eta_{s}}-\frac{2 R_{f, s} \kappa_{f, y}^{2} \eta_{f} \lambda}{\kappa_{s, y} \sqrt{\eta_{s}}}-R_{f, s}^{2} \kappa_{f, y}^{2} \eta_{f} \lambda^{2}\right)+R_{f, s}
$$

Here, also the approximation of the heater width being larger than the film thickness is assumed. For our setup, material combination and temperature range all the above mentioned geometrical conditions are well fulfilled. In order to further simplify and solve Eq. (s5) for $\Delta \mathrm{T}_{\mathrm{f}}$ we restrict the wave vector $\lambda$ in $x$ direction to the physically important range of $\lambda \leq(2 b)^{-1}$, where $(2 b)^{-1}$ is the reciprocal heater linewidth. We also know that the thermal boundary resistance between film and substrate is only one contribution to all Kapitza resistances in the measurement stack. Hence, $R_{f, s}<R_{K}$ and therefore we take $R_{K}$ as a maximum possible value for $R_{f, s}$. Numerical analysis shows that both terms containing $\lambda$ are very small and can be thus neglected. Furthermore, we note that due to a high ratio of substrate to film thermal conductivities in the $\mathrm{Sb}_{2} \mathrm{Te}_{3} / \mathrm{Al}_{2} \mathrm{O}_{3}$ system, the remaining correction term is generally rather small. With these approximations in Eq. ( $\mathrm{s} 5)$, the function $\Gamma\left(\mathrm{K}, \mathrm{Bi}_{\mathrm{f}}, \mathrm{Hr}\right)$ in $\mathrm{Eq}$. (s4) can be solved for the thermal offset of the film, only (Eq. (s6)) and we finally obtain the used equations for calculating $\kappa_{\mathrm{f}, \mathrm{y}}$ from the measured thermal offsets (s7a) and (s7b), which are shown in the main document as Equations (1b) and (1c).

$$
\begin{aligned}
& \Delta \mathrm{T}_{\mathrm{f}}=\frac{\mathrm{t}_{\mathrm{f}}}{\kappa_{\mathrm{f}, \mathrm{y}}} \frac{P}{l 2 b}\left(1-\frac{\kappa_{f, y}^{2} \eta_{f}}{\kappa_{s, y}^{2} \eta_{s}}\right) \\
& \kappa_{\mathrm{f}, \mathrm{y}}=\sqrt{\frac{1}{4} \Xi^{2}+\frac{\eta_{s}}{\eta_{f}} \cdot \kappa_{s, y}^{2}}-\frac{1}{2} \Xi \\
& \Xi=\frac{\eta_{s}}{\eta_{f}} \cdot \frac{2 b l}{t_{f} P} \cdot \Delta T_{f} \cdot \kappa_{s, y}^{2}
\end{aligned}
$$




\section{FIGURES}
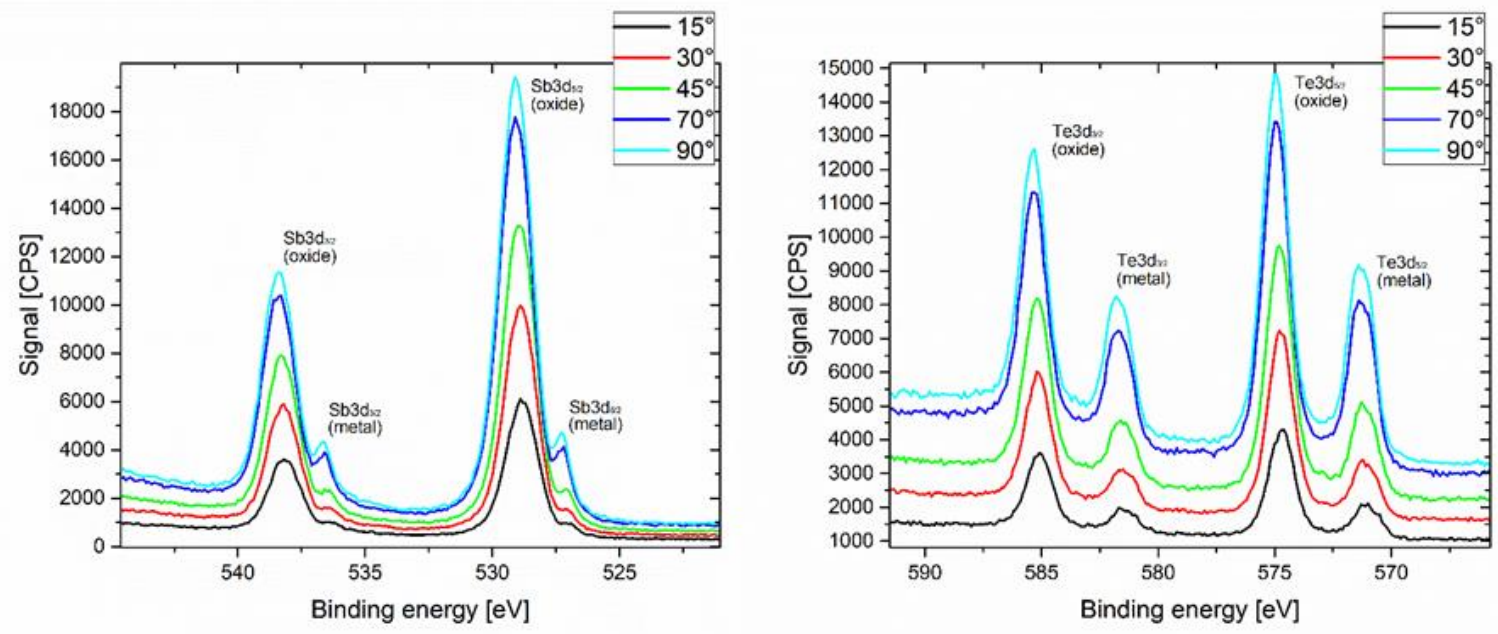

FIG. S1. Angle-dependent Sb 3d (left) and Te 3d (right) XPS core level spectra obtained using a Versaprobe II ${ }^{\mathrm{TM}}$ by UlvacPhi with monochromatic Al-K $\mathrm{K}_{\alpha}$ light at $1486.6 \mathrm{eV}$ photon energy. The spectra were used to calculate the thickness of the oxidized surface layer to $3.2 \pm 1.1 \mathrm{~nm}$.

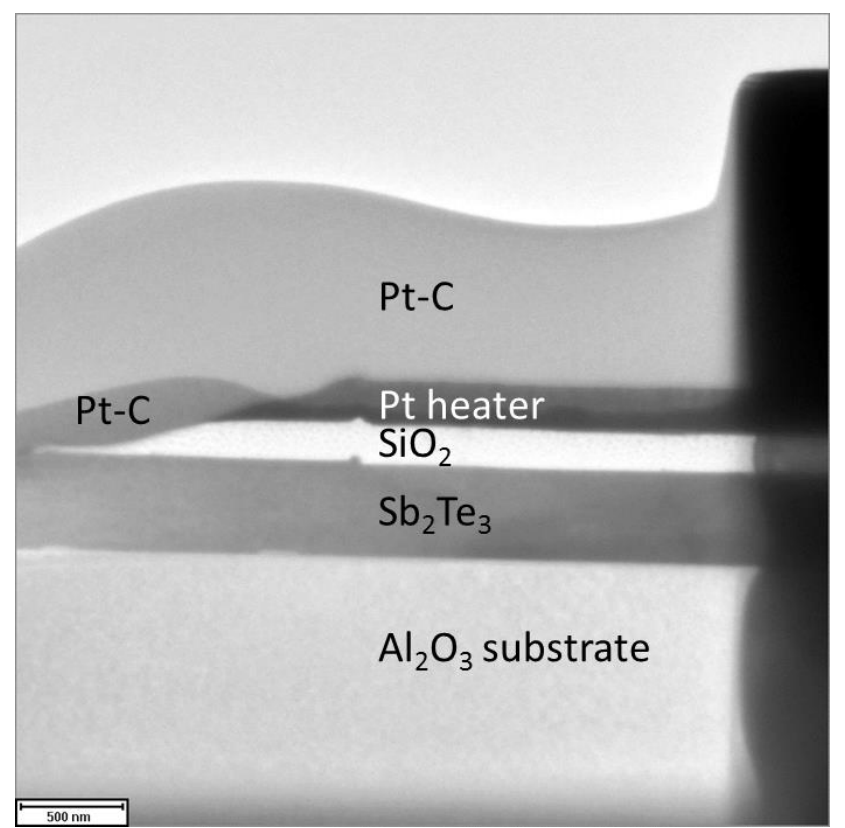

FIG. S2. Cross section TEM image of ion beam sputtered $\mathrm{SiO}_{2}$ insulation layer and Pt heater, taken at a Philips / FEI CM30. 


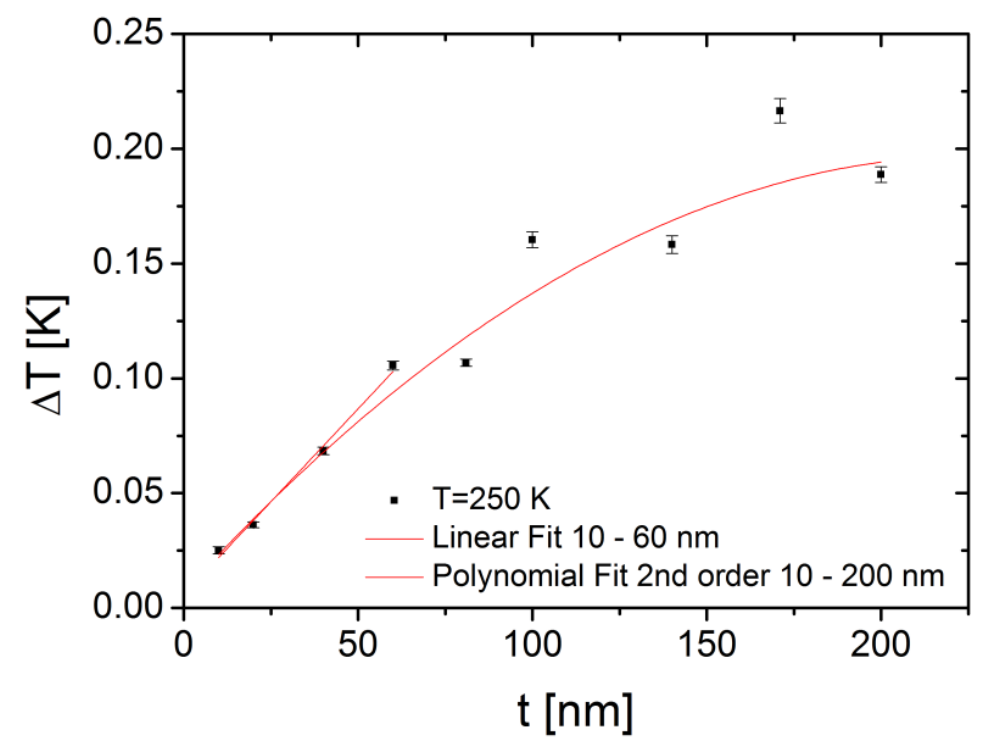

FIG. S3. $\mathrm{SiO}_{2}$ thickness series: $\Delta \mathrm{T}$ offsets for different $\mathrm{SiO}_{2}$ thicknesses at $250 \mathrm{~K}$. Linear and polynomial fits are represented as red lines. Linear fit: $y=0.0058+0.00162 x$. Polynomial fit: $\mathrm{y}=0.00704+0.00166 \mathrm{x}-0.00000364315 \mathrm{x}^{2}$.

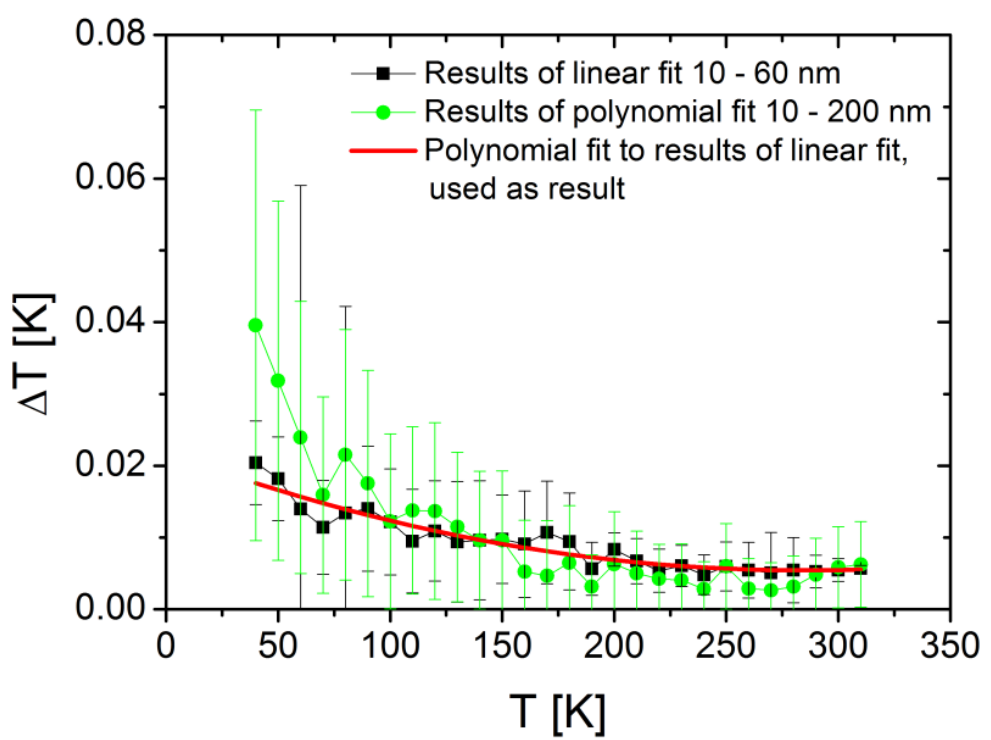

FIG. S4. Determination of the temperature offset related to the Kapitza A resistance from the $\mathrm{SiO}_{2}$ thickness series. Both the results of linear and polynomial fits are presented. The error for the polynomial fit in FIG. S3 is larger due to larger scattering of the $\Delta \mathrm{T}$ at large thicknesses. The red line shows a polynomial fit $2^{\text {nd }}$ order to the results of the linear fit as a guide to the eye and for subtraction of the Kapitza A resistance from thermal conductivity data of the entire film stack. 


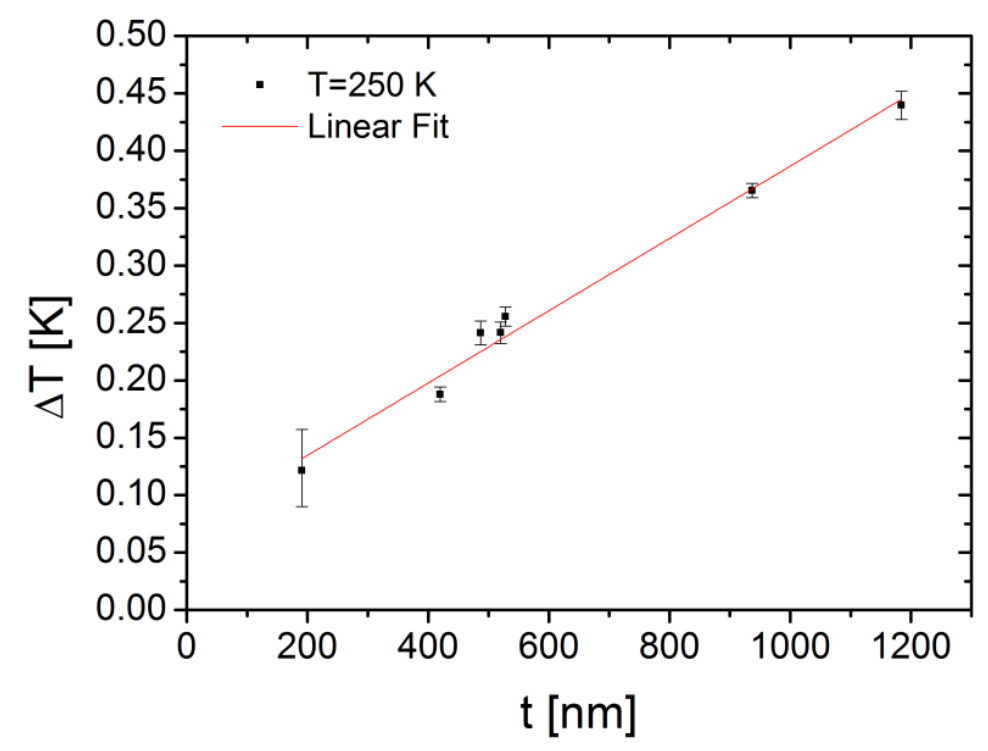

FIG. S5. $\mathrm{Sb}_{2} \mathrm{Te}_{3}$ thickness series for the determination of the Kapitza B resistance: $\Delta \mathrm{T}$ offsets for different $\mathrm{Sb}_{2} \mathrm{Te}_{3}$ thicknesses at $\mathrm{T}=250 \mathrm{~K}$. The red line shows the linear fit according to $\mathrm{y}=0.07175+0.00031497 \mathrm{x}$.

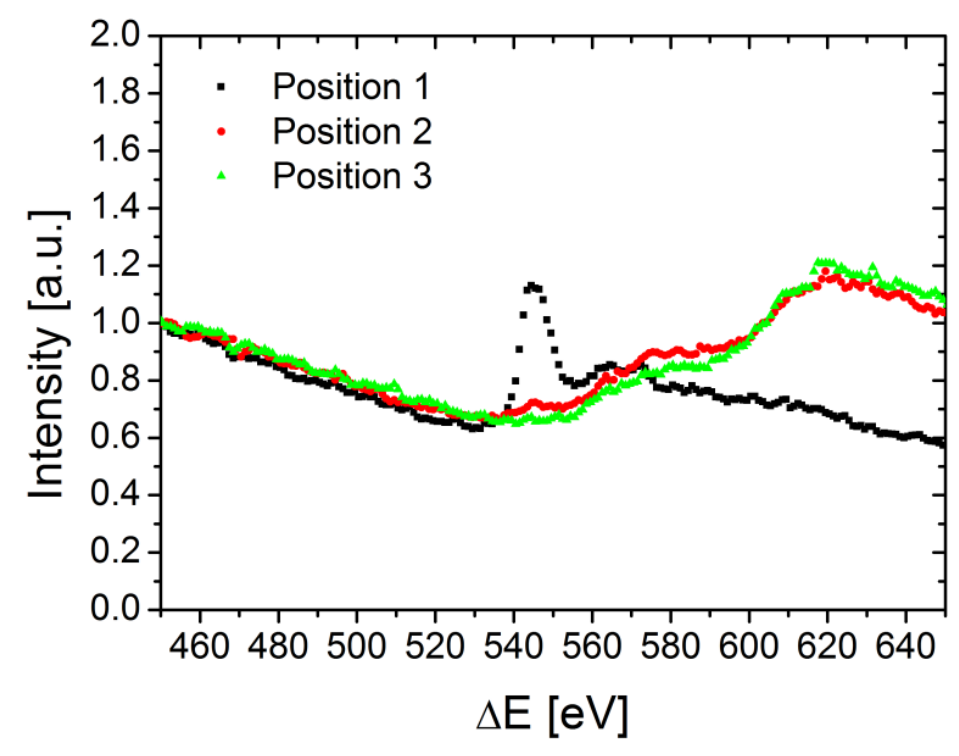

FIG. S6. High electron energy loss spectra (EELS) in the range of 450 to $650 \mathrm{eV}$. Raw data before subtraction of the background. Positions refer to those in FIG. 6. 


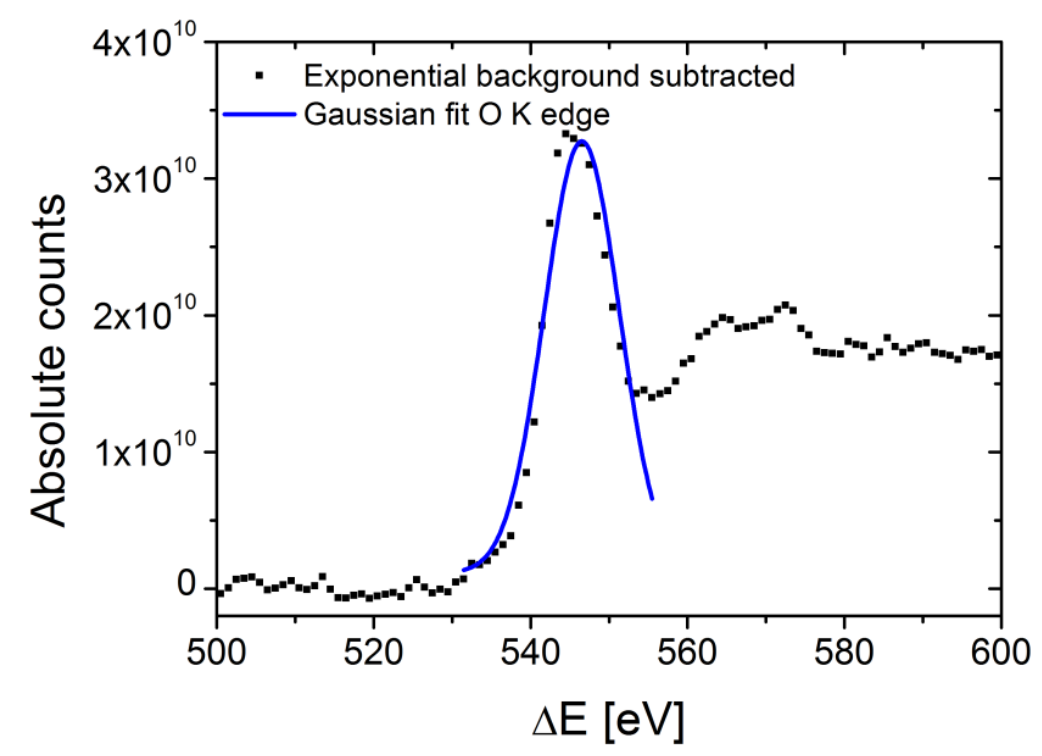

FIG. S7. EELS at position 1 after subtraction of an exponential background and showing a Gaussian fit for $\mathrm{O} \mathrm{K}$ edge.

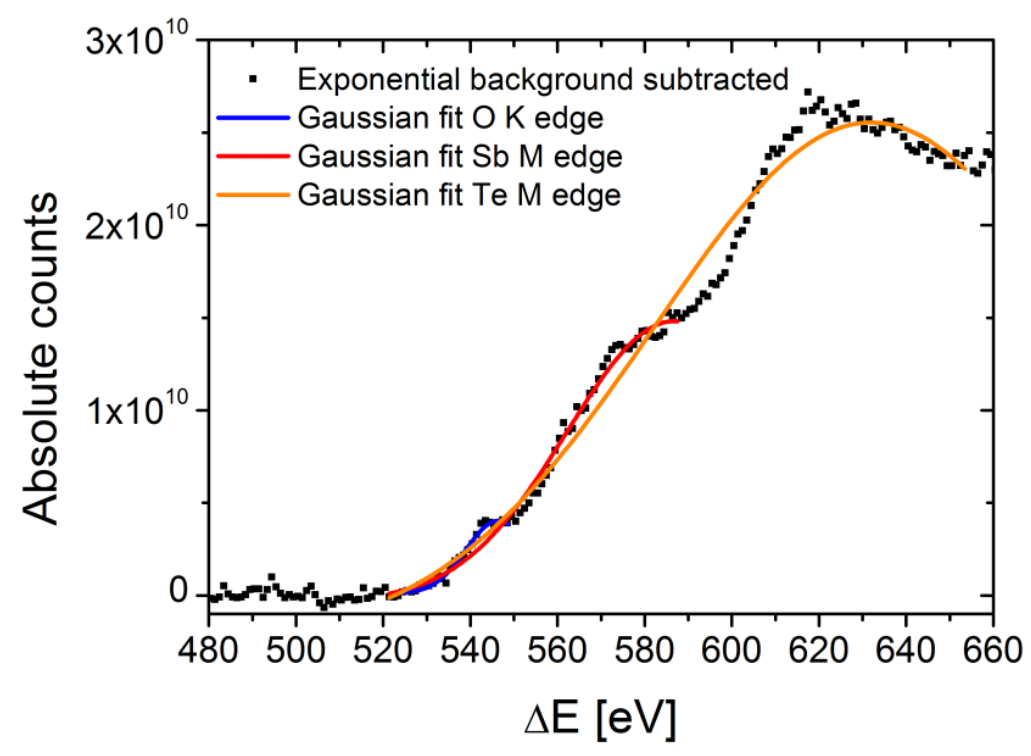

FIG. S8. EELS at position 2 after subtraction of an exponential background and showing Gaussian fits for the $\mathrm{O} \mathrm{K}, \mathrm{Sb} \mathrm{M}$ and Te M edges. 


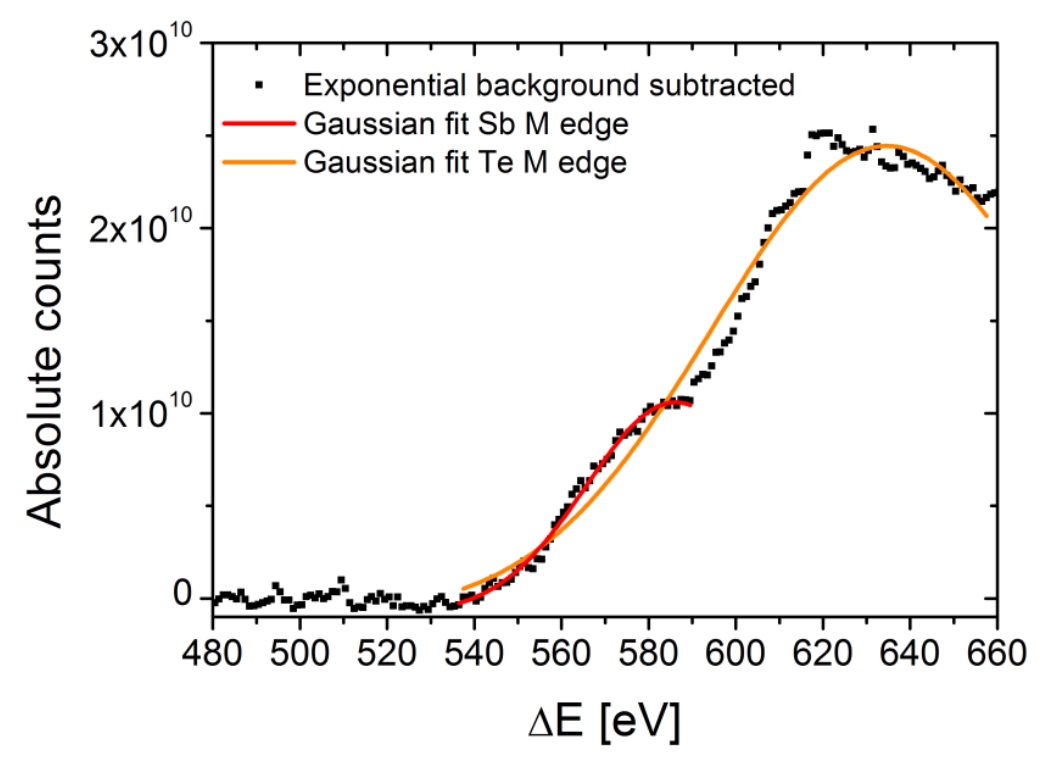

FIG. S9. EELS at position 3 after subtraction of an exponential background and showing Gaussian fits for the $\mathrm{Sb} \mathrm{M}$ and Te M edges.

\footnotetext{
${ }^{1}$ T. Tong and A. Majumdar, Rev. Sci. Instrum. 77, 104902 (2006).

${ }^{2}$ D. G. Cahill, M. Katiyar, and J. R. Abelson, Phys. Rev. B 50(9), 6077 (1994).
} 



\section{CHAPTER 5}

\section{Transition into a phonon glass in crystalline thermoelec- tric $\left(\mathrm{Sb}_{1-\mathrm{x}} \mathrm{Bi}_{\mathrm{x}}\right)_{2} \mathrm{Te}_{3}$ films}

This chapter consists of the manuscript

Transition into a phonon glass in crystalline thermoelectric $\left(\mathrm{Sb}_{1-\mathrm{x}} \mathrm{Bi}_{\mathrm{x}}\right)_{2} \mathrm{Te}_{3}$ films

F. Rieger ${ }^{1}$, V. Roddatis ${ }^{1}$, K. Kaiser ${ }^{2}$, G. Bendt ${ }^{2}$, S. Schulz ${ }^{2}$, and C. Jooss ${ }^{1}$.

${ }^{1}$ Institute of Material Physics, University of Göttingen, 37077 Göttingen, Germany

${ }^{2}$ Institute of Inorganic Chemistry and Center for Nanointegration Duisburg-Essen (Cenide), University of Duisburg - Essen, 45141 Essen, Germany

This chapter also includes the corresponding supplemental material. Both documents are modified concerning font sizes and line spacing. The manuscript is ready for submission to a peer-reviewed journal.

For consistency reasons the manuscript is called "Publication 2" although it has not been published yet when this thesis was published. 



\title{
Transition into a phonon glass in crystalline thermoelectric $\left(\mathrm{Sb}_{1-\mathrm{x}} \mathrm{Bi}_{\mathrm{x}}\right)_{2} \mathbf{T e}_{3}$ films
}

\author{
F. Rieger ${ }^{1}$, V. Roddatis ${ }^{1}$, K. Kaiser ${ }^{2}$, G. Bendt ${ }^{2}$, S. Schulz ${ }^{2}$, and C. Jooss ${ }^{1}$ \\ ${ }^{1}$ Institute of Material Physics, University of Göttingen, 37077 Göttingen, Germany \\ ${ }^{2}$ Institute of Inorganic Chemistry and Center for Nanointegration Duisburg-Essen \\ (Cenide), University of Duisburg-Essen, 45141 Essen, Germany
}

Gaining fundamental insights into phonon interactions is of high importance for the improvement of thermoelectric materials. The particular challenge is to enable a phonon glass state with low thermal conductivity in crystalline materials with high electron conductivity. We present here the relation between atomic structure and c-axis lattice thermal conductivity $\kappa_{c, l}(T)$ of epitaxially grown nearly single crystalline $\left(\mathrm{Sb}_{1-\mathrm{x}} \mathrm{Bi}_{\mathrm{x}}\right)_{2} \mathrm{Te}_{3}$ thin films. Aberration corrected high-resolution transmission electron microscopy shows a highly ordered crystalline lattice, with doping dependent statistical occupation of the $\mathrm{Sb}$ sublattice by $\mathrm{Bi}$ atoms and a very low density of planar defects. The observed strong decrease of $\kappa_{c, l}(T)$ with doping is due to an increase both of the Rayleigh scattering rate at point defects as well as of the phononphonon scattering rate. For $x=0.24$ a transition to a low almost temperature independent $\kappa_{c, l}(T)$ is observed, indicating a transition to a phonon glass state. The theoretical calculations reveal that the phonon mean free path is reduced below the phonon wavelength for the majority of phonon frequencies, suggesting a break down of the phonon approximation to heat transport due to the strongly anharmonic lattice.

\section{INTRODUCTION}

Binary pnictide chalcogenides with tetradymite crystal structure, such as $\mathrm{Bi}_{2} \mathrm{Te}_{3}$ and $\mathrm{Sb}_{2} \mathrm{Te}_{3}$, are semiconductors with a large thermoelectric Figure of merit, $Z T \sim 1$, close to room temperature ${ }^{[1]}$. Although they have been successfully applied in Peltier cooling and thermoelectric energy conversion since the $1950 \mathrm{~s}^{[2]}$, basic materials properties such as the nature of bonding, the impact of spin-orbit coupling ${ }^{[3]}$ on properties as well as their anisotropic thermal conductivities are not well understood. In particular, the origin of the relatively low values of the c-axis thermal conductivity with $\kappa_{c}=1.9 \mathrm{Wm}^{-1} \mathrm{~K}^{-1}$ for $\mathrm{Sb}_{2} \mathrm{Te}_{3}{ }^{[4]}$ and $\kappa_{c}=1.2 \mathrm{Wm}^{-1} \mathrm{~K}^{-1}$ for $\mathrm{Bi}_{2} \mathrm{Te}_{3}{ }^{[5]}$, and its strong further reduction in $\left(\mathrm{Sb}_{1-\mathrm{x}} \mathrm{Bi}_{\mathrm{x}}\right)_{2} \mathrm{Te}_{3}$ solid solutions remains elusive, since data obtained at nanostructured materials ${ }^{[6,7]}$ does not allow for separating out intrinsic and microstructural effects. 
Both $\mathrm{Bi}_{2} \mathrm{Te}_{3}$ and $\mathrm{Sb}_{2} \mathrm{Te}_{3}$ exhibit a layered rhombohedral lattice structure $(R \overline{3} m$, space group \#166). Along the c-direction, quintuple -[Te(I)-Pn-Te(II)-Pn-Te(I)]- stacks (Pn $=\mathrm{Bi}, \mathrm{Sb})$ are forming a unit cell, where $\mathrm{Te}(\mathrm{I})$ and $\mathrm{Te}(\mathrm{II})$ represent two types of differently bonded tellurium atoms. Whereas $\mathrm{Te}(\mathrm{II})$ is coordinated nearly octahedrally by $P n$ atoms, Te(I) has three $P n$ and three Te(I) as nearest neighbors. The bonding between the quintuple stacks, i.e. between $\mathrm{Te}(\mathrm{I})$ atoms is considered as of van der Waals ( $\mathrm{vdW}$ ) type and is the origin of easy cleavage of these materials perpendicular to the $c$-axis ${ }^{[8]}$. In addition, evidence for resonance bonding (RB) within the quintuple units due to sharing of electrons in unsaturated covalent bonds is obtained from theoretical considerations ${ }^{[9]}$. Both $\mathrm{vdW}$ and $\mathrm{RB}$ bonding can create soft lattices with pronounced anharmonicity of the bonding potential, leading to low phonon velocity as well as strong phonon-phonon interactions.

A publication of Venkatasubramanian et al. in the year 2001 ${ }^{[10]}$ on MOCVD-grown $\mathrm{Sb}_{2} \mathrm{Te}_{3} / \mathrm{Bi}_{2} \mathrm{Te}_{3}$ superlattices triggered various studies on improvement of thermoelectric performance in superlattices: It claimed a very high ZT value of $\sim 2.4$ at $300 \mathrm{~K}$ based on a remarkably low lattice thermal conductivity of $0.22 \mathrm{Wm}^{-1} \mathrm{~K}^{-1}$, determined by extrapolation of the total thermal conductivity of the superlattices to zero doping. ${ }^{[11]}$ To date this value could not be reproduced by other research groups. In addition, an instability of these superlattices at temperatures above room temperature was demonstrated ${ }^{[12,13,14]}$, indicating that low c-axis thermal conductivity is due to interdiffusion. Indeed, thermal decay of $\mathrm{Sb}_{2} \mathrm{Te}_{3} / \mathrm{Bi}_{2} \mathrm{Te}_{3}$ superlattices revealed a reduction of the cross-plane thermal conductivity down to $0.6 \mathrm{Wm}^{-1} \mathrm{~K}^{-1}$, whereas no reduction was found in stable heterostructures. ${ }^{[15]}$

In recent years, experimental ${ }^{[16,17]}$ and theoretical studies ${ }^{[18,19,20]}$ elucidated a quite pronounced anharmonicity of the atomic bonding potential in $\mathrm{Bi}_{2} \mathrm{Te}_{3}$ as well as in $\mathrm{Sb}_{2} \mathrm{Te}_{3}$. This can be expressed in the Grüneisen parameter $\gamma_{\mathrm{G}}=\alpha_{\mathrm{V}} /\left(\mathrm{C}_{\mathrm{V}} \mathrm{K}_{\mathrm{T}}\right)$, which compares the measured volume expansivity to the product of the heat capacity $\mathrm{C}_{\mathrm{V}}$ and the lattice compressibility $\mathrm{K}_{\mathrm{T}}$. Measurements give rather moderate values for the binary systems $\left(\gamma_{G}=1.7\right.$ for $\mathrm{Sb}_{2} \mathrm{Te}_{3}$ and $\gamma_{G}=1.5$ for $\mathrm{Bi}_{2} \mathrm{Te}_{3}$ at $\left.295 \mathrm{~K}^{[21]}\right)$. The observation of a doping induced softening of a transverse optical (TO) phonon mode by Se substitution of $\mathrm{Te}^{[16,21]}$ and the resulting decrease of the phonon bandgap between acoustic $(\mathrm{A})$ and optical $(\mathrm{O})$ modes suggests that a strong increase of phononphonon interactions in $(\mathrm{A}, \mathrm{O}, \mathrm{O})$ and $(\mathrm{A}, \mathrm{A}, \mathrm{O})$ three phonon processes. Such a scenario is discussed in a recent publication as a key factor for low thermal conductivities in $\mathrm{RB}$ material systems ${ }^{[22]}$. In addition, spin-orbit interactions (SOI) can shift down the energy of $\mathrm{A}$ as well as $\mathrm{TO}$ modes in $\mathrm{Sb}_{2} \mathrm{Te}_{3}$ and thus gives rise to a higher density of phonon states at low energies ${ }^{[23]}$. Consequently, studying point disorder and anharmonicity induced thermal disorder of lattice planes in the soft direction (c-axis) 
as a function of doping is of high interest for unraveling the mechanisms of phonon transport in these thermoelectric materials systems.

Our study provides thus a correlation between atomic structure of crystalline epitaxial $\left(\mathrm{Sb}_{1-\mathrm{x}} \mathrm{Bi}_{\mathrm{x}}\right)_{2} \mathrm{Te}_{3}$ films obtained by high resolution transmission electron microscopy to careful temperature dependent measurements of c-axis lattice thermal conductivity $\kappa_{c, l}(x, T)$. While $\kappa(T)$ data are available for nanocrystalline $\mathrm{Sb}_{2} \mathrm{Te}_{3}{ }^{[24]}$ and nanocrystalline $\left(\mathrm{Sb}_{1-\mathrm{x}} \mathrm{Bi}_{\mathrm{x}}\right)_{2} \mathrm{Te}_{3}$ samples ${ }^{6,7}$ over a wide temperature range, $\kappa(T)$ data for specific crystallographic directions is only available on $\mathrm{Sb}_{2} \mathrm{Te}_{3}$ single crystals for the a,b-plane ${ }^{[25,26]}$. We are not aware of any $\kappa_{c}(T)$ measurements for $\left(\mathrm{Sb}_{1-\mathrm{x}} \mathrm{Bi}_{\mathrm{x}}\right)_{2} \mathrm{Te}_{3}$ crystals. Theoretical calculations of $\kappa_{c, l}(T)$ are performed based on the Callaway model ${ }^{[27]}$, using complete phonon dispersions for the calculation of the phonon velocity and density of states (see method section and supplementary material). These calculations give access to the doping dependent change of phonon-phonon and Rayleigh point defect scattering rates. The transition to an almost temperature independent low $\kappa_{c, l}(T)$ for $x=0.24$ and $T=160-300 \mathrm{~K}$ is described within the minimum lattice thermal conductivity model of Cahill et al. ${ }^{[28]}$, a modification of the model of Slack ${ }^{[29]}$, establishing the transition to a phonon glass state for the phonon transport along c-axis.

\section{EXPERIMENTAL}

\section{A. Sample preparation}

Epitaxial $\left(\mathrm{Sb}_{1-\mathrm{x}} \mathrm{Bi}_{\mathrm{x}}\right)_{2} \mathrm{Te}_{3}$ thin films were deposited on $\mathrm{Al}_{2} \mathrm{O}_{3}(0001)$ [10x10x0.5 mm] substrates using a vertical physical vapour deposition (PVD) system that was previously described elsewhere ${ }^{[30]}$. Mixtures of commercial $\mathrm{Sb}_{2} \mathrm{Te}_{3}(99.999 \%$ abcr) and $\mathrm{Bi}_{2} \mathrm{Te}_{3}$ powders $(99.99 \%$ abcr) were used in various ratios as precursor material. The film deposition was performed at constant substrate $\left(330{ }^{\circ} \mathrm{C}\right)$ and evaporator temperatures $\left(570{ }^{\circ} \mathrm{C}\right)$ for epitaxial $\mathrm{Sb}_{2} \mathrm{Te}_{3}$ and $\left(\mathrm{Sb}_{1-\mathrm{x}} \mathrm{Bi}_{\mathrm{x}}\right)_{2} \mathrm{Te}_{3}$ thin films on $\mathrm{Al}_{2} \mathrm{O}_{3}$ (0001) substrates. Successful growth of c-oriented highly crystalline films was possible in the doping range $x=0,0.07,0.12,0.24$, respectively, at a working pressure of 20 mbar. In addition, highly crystalline, epitaxial $\mathrm{Bi}_{2} \mathrm{Te}_{3}$ films were grown on $\mathrm{Al}_{2} \mathrm{O}_{3}$ (0001) substrates at a substrate temperature of $290{ }^{\circ} \mathrm{C}$ and evaporator temperature of $605{ }^{\circ} \mathrm{C}$ under high vacuum conditions $\left(10^{-4} \mathrm{mbar}\right)$. The growth parameters are summarized in Table S1 in the supplementary material. XRD studies were performed with a Bruker D8 Advance powder diffractometer in a Bragg-Brentano geometry, using a $\mathrm{Cu}-\mathrm{K} \alpha$ radiation $(\lambda=1.5418 \AA)$. The chemical composition of the resulting binary and ternary material films was determined by EDX and the results are given in Table S2. 


\section{B. HRSTEM investigations}

Sample preparation for TEM analysis was carried out by a Focused Ion Beam (FIB), using a FEI Nova NanoLab 600. Due to the pronounced beam sensitivity of the vdW layered material, a dedicated preparation procedure was applied to obtain high quality TEM lamellas with minimized thin amorphous top layer. Rough cutting and thinning steps were done at $30 \mathrm{kV}$ followed by a thinning step at a beam voltage of $5 \mathrm{kV}$. High-

Resolution Transmission Microscopy (HRTEM) and Scanning Transmission Microscopy (STEM) investigations were performed at an aberration $\left(C_{\mathrm{S}}\right)$ corrected FEI Titan 80-300 kV operated at $300 \mathrm{kV}$. HRTEM studies were done for all differently doped samples. An interface analysis of the $\mathrm{Sb}_{2} \mathrm{Te}_{3}$ film via HRTEM, STEM and Electron Energy Loss Spectroscopy (EELS) is published in ref. ${ }^{[4]}$.

\section{HRSTEM simulations}

HRSTEM images show random distribution of individual atomic columns or parts of the corresponding lattice planes occupied with $\mathrm{Sb}$ and $\mathrm{Bi}$ with the enhanced contrast. This effect was reproduced by the HRSTEM image simulations using a QSTEM software package ${ }^{[31]}$. A box of $8 \times 8 \times 15 \mathrm{~nm}$ was filled with $\mathrm{Sb}_{2} \mathrm{Te}_{3}$ or $\mathrm{Bi}_{2} \mathrm{Te}_{3}$ unit cells. In order to reproduce mixed compositions, randomly selected 12 or 24 percent of $\mathrm{Sb}$ atoms were replaced with $\mathrm{Bi}$ atoms. Simulation parameters were chosen as listed in Table S3.

\section{Thermal conductivity measurements}

The cross-plane thermal conductivity $\kappa_{c}(T)$ of different samples was measured in a temperature range from 100 to $300 \mathrm{~K}$. These measurements were performed by a selfbuilt $3 \omega$ setup. The thermal conductivity data has been obtained by data analysis, where the contributions of the ion beam sputtered $\mathrm{SiO}_{2}$ layer for electrical insulation of the Pt-heater structure as well as the thermal boundary resistances (Kapitza resistances) have been carefully determined and subtracted. This has been done on the basis of two layer thickness series. For details regarding Kapitza resistance, sample geometry and the applied $3 \omega$ evaluation see ref. ${ }^{[4]}$. The formulas used for considering horizontal heat spread in the film require values for the anisotropy $\eta$ of $\kappa$. The used value for $x=0$ was $\eta=2.3^{[4]}$ and $\eta=1.59$ for $x=0.25^{[32]}$. For $x=0.07-0.24$ an interpolation of $\eta(x)$ between $x=0$ and $x=0.25$ has been used and for $x=1$, the anisotropy of $\eta=2.3$ has been obtained from ref. ${ }^{[33]}$.

\section{E. Lattice thermal conductivity calculations}

In order to develop a better understanding of the fundamental mechanisms that determine the phonon heat transport in $\left(\mathrm{Sb}_{1-\mathrm{x}} \mathrm{Bi}_{\mathrm{x}}\right)_{2} \mathrm{Te}_{3}$, lattice thermal conductivity calculations and fitting to the experimental data was performed using Matlab. For every temperature, the sum over all phonon modes $n$ was calculated by 


$$
\kappa_{c, l}(T)=\sum_{n 1}^{n 2} \int_{\omega_{\min }}^{\omega_{\max }} g(\omega) C(\omega, T) v_{n}(\omega) l_{m f p}(\omega, T) d \omega,
$$

where $g(\omega)$ is the density of states per angular frequency $\omega, v_{n}(\omega)$ is the velocity of sound of a specific phonon mode $n, C(\omega, T)$ the specific heat and $l_{m f p}(\omega, T)$ the frequency and temperature dependent mean free path. The density of states $g(\omega)$ was obtained from experimental Neutron Incoherent Scattering (NIS) data ${ }^{[34]}$ of $\mathrm{Sb}_{2} \mathrm{Te}_{3}$ and $\mathrm{Bi}_{2} \mathrm{Te}_{3}$, fitted by polynomials, see Fig. S1 in the supplementary material. In order to get the absolute values needed for our calculations, the total density of states curve $g(\omega)$ was scaled to an absolute value of

$$
g\left(\omega_{D}\right)=\frac{3}{2 \pi^{2} v_{c}^{3}} \cdot \omega_{D}^{2}
$$

at the Debye frequency $\omega_{D}$, which is given by

$$
\omega_{D}=\frac{k \Theta_{D}}{\hbar}=21,6 \mathrm{THz}
$$

This value was taken for all samples because of $\Theta_{D}=165 \mathrm{~K}$ for $\mathrm{Sb}_{2} \mathrm{Te}_{3}{ }^{[35]}$ and $\mathrm{Bi}_{2} \mathrm{Te}_{3}{ }^{[36]}$. For the single modes the used $g(\omega)$ in eq. (1) were divided by 9 since only phonons along one direction contribute and (in the most relevant low frequency regime) three modes add up to the total $g(\omega)$.

The specific heat capacity per phonon $C(\omega, T)$ is expressed by

$$
C(\omega, T)=\frac{(\hbar \omega)^{2}}{k_{B} T^{2}} \frac{\exp \left(\frac{\hbar \omega}{k_{B} T}\right)}{\left(\exp \left(\frac{\hbar \omega}{k_{B} T}\right)-1\right)^{2}} .
$$

The velocity of sound $v_{n}(\omega)$ of a specific phonon mode $n$ at a specific angular frequency $\omega$ as well as the minimal and maximal frequencies of a specific phonon mode, $\omega_{\min }$ and $\omega_{\max }$, were obtained from first principles calculations of the phonon dispersion relations of $\mathrm{Sb}_{2} \mathrm{Te}_{3}{ }^{[23]}$ and $\mathrm{Bi}_{2} \mathrm{Te}_{3}{ }^{[18]}$, respectively. For all samples, except $x=1$, the phonon dispersion relations of $\mathrm{Sb}_{2} \mathrm{Te}_{3}$ were used. This is justified from the experimental observation that the solid solutions do not alter the symmetry of the crystal lattice and the qualitative similarity of the phonon dispersion relations for $x=0$ and $x=1$. The obtained average velocity of acoustic phonon modes is in good agreement with sound velocities $v_{c}$ deduced from Stiffness matrices ${ }^{[37]}$ and mass densities ${ }^{[38]}$, yielding $v_{c}=1383 \mathrm{~ms}^{-1}$ for $\mathrm{Sb}_{2} \mathrm{Te}_{3}$ and $v_{c}=1057 \mathrm{~ms}^{-1}$ for $\mathrm{Bi}_{2} \mathrm{Te}_{3}$, see Fig. S2 in the supplementary material.

For the total phonon mean free path $l_{m f p}(\omega, T)$ we used a combination of Umklapp scattering and Rayleigh scattering, summed up according to the Matthiessen rule:

$$
l_{m f p}(\omega, T)=\frac{1}{\frac{1}{l_{m f p, U}}+\frac{1}{l_{m f p, R}}}
$$

For Umklapp and Rayleigh scattering we have used the following analytical approximations $^{[27]}$ :

$$
\begin{aligned}
& l_{m f p, U}=A\left(\frac{k_{B}}{\hbar \omega}\right)^{2} \frac{1}{T} \exp \left(\frac{\Theta_{D}}{3 T}\right) \\
& l_{m f p, R}=B\left(\frac{k_{B}}{\hbar \omega}\right)^{4}
\end{aligned}
$$


The parameters $A$ and $B$ are obtained by fitting the calculated $\kappa_{c, l}(T)$ curve to the experimental data. These semi-empirical equations have been justified by comparison with experimental thermal conductivity in semiconductors.

Based on the Debye model, Cahill et al. ${ }^{[28]}$ assumed a random walk between localized quantum mechanical oscillators to determine the minimum lattice thermal conductivity. We use the resulting expression, eq. (8), to calculate the minimum lattice thermal conductivities of $\mathrm{Sb}_{2} \mathrm{Te}_{3}$ and $\mathrm{Bi}_{2} \mathrm{Te}_{3}$, respectively. This expression only requires the density of atoms $n$ and the speed of sound $v_{i}$ for two transverse and one longitudinal mode. Eq. (9) determines the respective cutoff frequencies $\mathrm{k}_{\mathrm{B}} \Theta_{i}=\hbar \omega$ using the Debye approximation.

$$
\begin{aligned}
& \kappa_{\text {min }}=\left(\frac{\pi}{6}\right)^{\frac{1}{3}} k_{B} n^{\frac{2}{3}} \sum_{i} v_{i}\left(\frac{T}{\Theta_{i}}\right)^{2} \int_{0}^{\frac{\Theta_{i}}{T}} \frac{x^{3} e^{x}}{\left(e^{x}-1\right)^{2}} d x \\
& \Theta_{i}=v_{i}\left(\frac{\hbar}{k_{B}}\right)\left(6 \pi^{2} n\right)^{\frac{1}{3}}
\end{aligned}
$$

\section{RESULTS}

\section{A. Crystallinity of epitaxial $\left(\mathrm{Sb}_{1-\mathrm{x}} \mathrm{Bi}_{\mathrm{x}}\right)_{2} \mathrm{Te}_{3}$ thin films}

The c-axis thermal conductivity study is performed at a series of epitaxial c-axis oriented $\left(\mathrm{Sb}_{1-\mathrm{x}} \mathrm{Bi}_{\mathrm{x}}\right)_{2} \mathrm{Te}_{3}(x=0,0.07,0.12,0.24,1)$ thin films grown by physical vapor deposition (PVD). The epitaxial growth of phase pure $c$-orientated rhombohedral tetradymite-type films on (0001) $\mathrm{Al}_{2} \mathrm{O}_{3}$ substrates is confirmed by $\mathrm{XRD}$, showing only the $00 l(l=3,6,9,12 \ldots)$ reflections, see Fig. S3 in the supplementary material. Sharp peaks with a full width of half maximum of $\Delta \omega<0.2^{\circ}$ confirm high crystallinity. Exemplary SEM pictures of the $\mathrm{Sb}_{2} \mathrm{Te}_{3}(x=0)$ film and of the $\mathrm{Bi}_{2} \mathrm{Te}_{3}(x=1)$ film reveal smooth surfaces as shown in Fig. S4.

The high perfection of the epitaxial growth is confirmed by HRTEM and STEM, see Fig. 1. The size of crystal twin domains is usually larger than $500 \mathrm{~nm}$. The films have also a low density of other macroscopic defects such as antiphase boundaries and stacking faults. Some examples are shown in Fig. S5. Very few antiphase boundaries are observed, i.e. planar defects which are formed by the shift of atomic planes formed by $\mathrm{Sb}$ and $\mathrm{Te}$ atoms. The density of stacking faults is very low close to the substrate surface (average distance $50-100 \mathrm{~nm}$ ), and increases slightly with increase of doping from $x=0$ to $x=0.24$ as well as with increasing distance from the substrate. Close to the upper surface of the films, the average distance can approach $10 \mathrm{~nm}$. This may be due to a small temperature gradient during film growth in the thermally poor conducting films. The $\mathrm{Bi}_{2} \mathrm{Te}_{3}$ film shows slightly more extended defects compared to the Bi-doped $\mathrm{Sb}_{2} \mathrm{Te}_{3}$ films, especially in the upper surface region. 


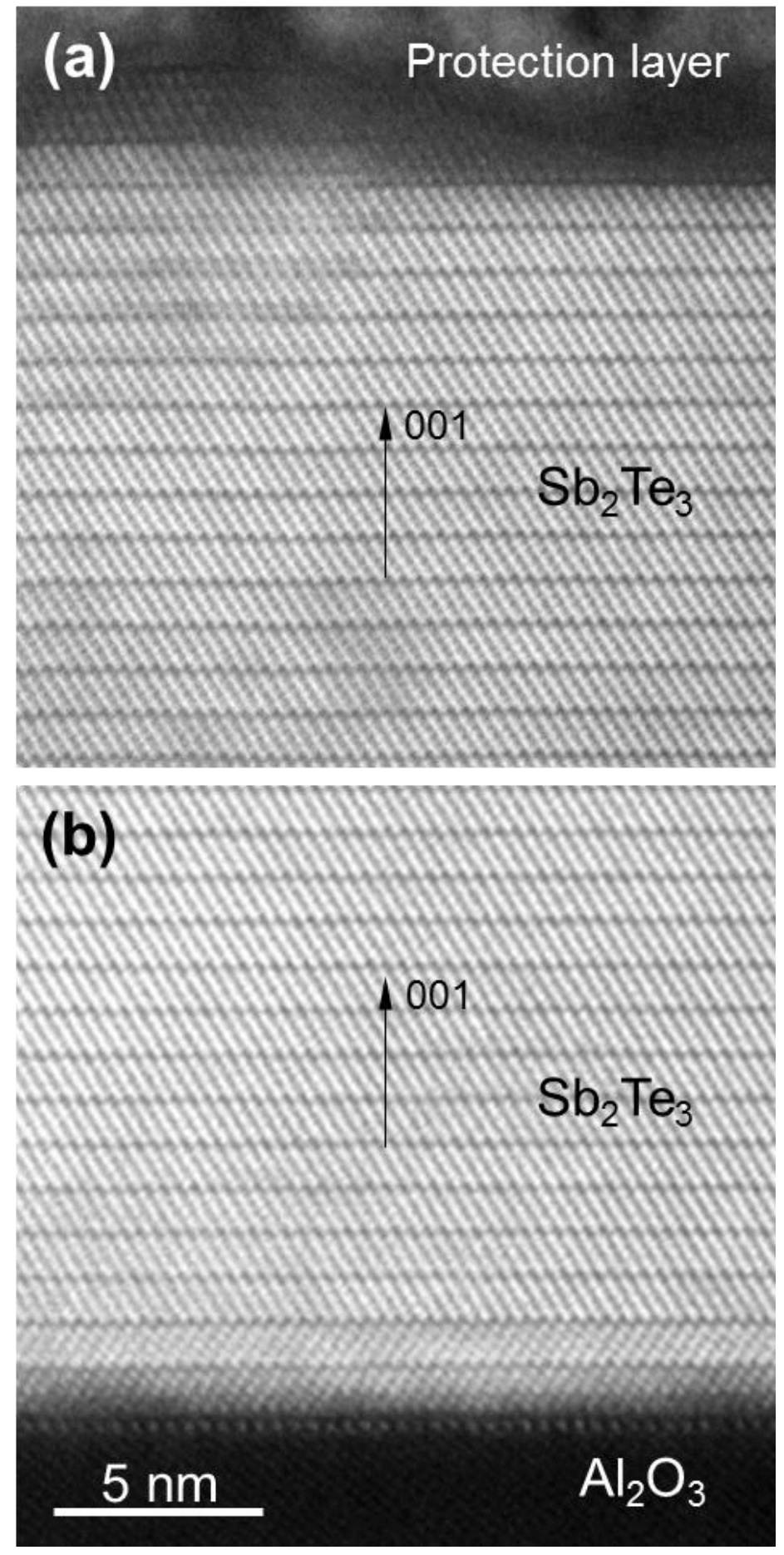

FIG. 1. HRSTEM images of $\mathrm{Sb}_{2} \mathrm{Te}_{3} / \mathrm{Al}_{2} \mathrm{O}_{3}$ interface and top surface of $\mathrm{Sb}_{2} \mathrm{Te}_{3}$ film. The interface reconstruction and twinned areas (2-3 u.c.) close to the $\mathrm{Sb}_{2} \mathrm{Te}_{3} / \mathrm{Al}_{2} \mathrm{O}_{3}$ interface is sometimes observed. STEM imaging reveals excellent crystallinity of the films, furthermore supported by XRD and local selected area electron diffraction patterns shown in the supplementary material. 

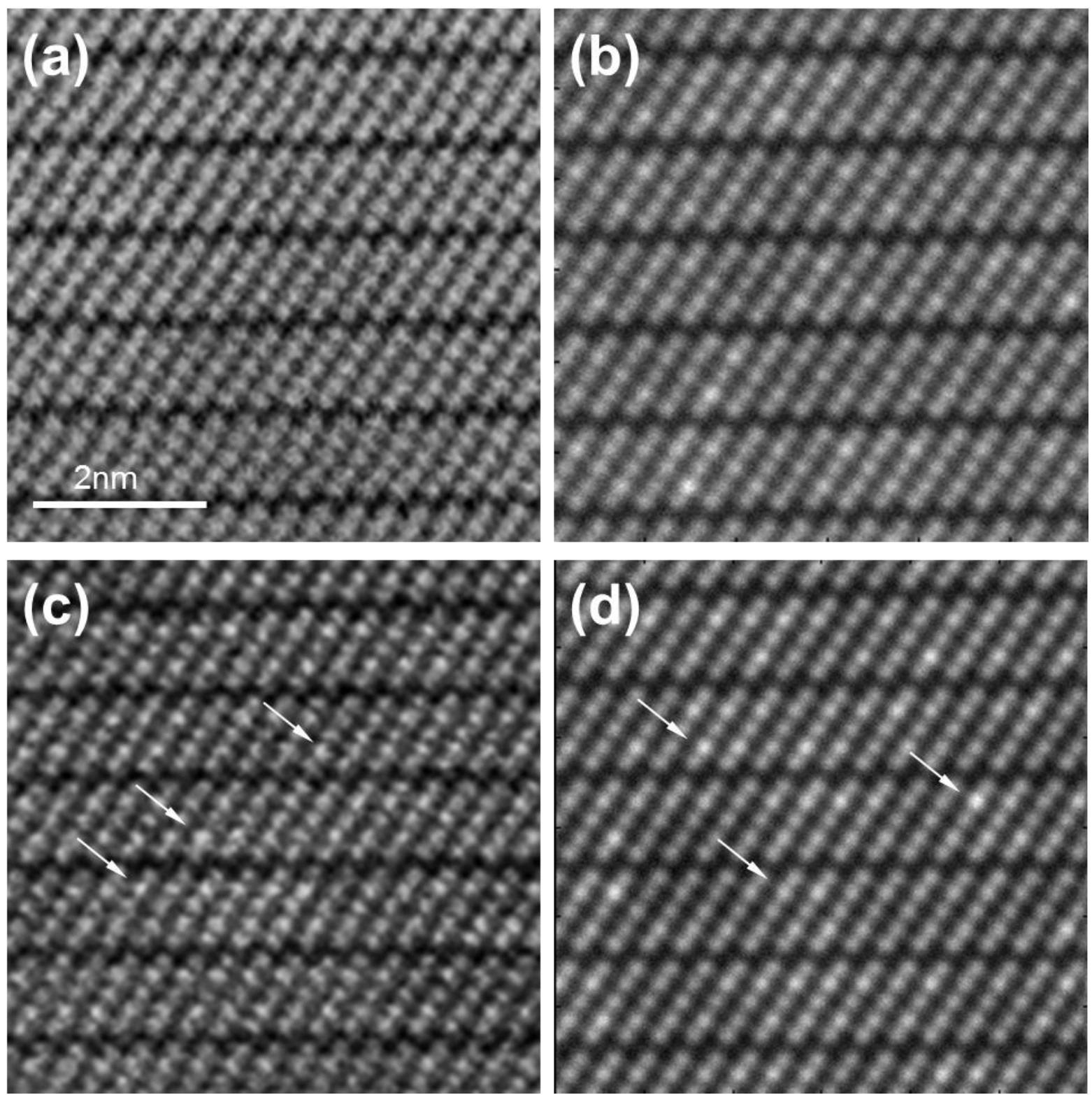

FIG. 2. Experimental and calculated HRSTEM HAADF images (zone axis [010]) of $\mathrm{Sb}_{0.88} \mathrm{Bi}_{0.12} \mathrm{Te}_{3}$ (a) and (b), $\mathrm{Sb}_{0.76} \mathrm{Bi}_{0.24} \mathrm{Te}_{3}$ (c) and (d) respectively. A random substitution $\mathrm{Bi}$ atoms is used in the simulated image of $\mathrm{Sb}_{0.88} \mathrm{Bi}_{0.12} \mathrm{Te}_{3}$ films, leading to random appearance of individual bright spots due to atomic columns with higher Bi occupation. The occupation of $\mathrm{Sb}$ sites by $\mathrm{Bi}$ atoms gives rise to an increased intensity of the $P n$ sites in the quintuple $[\mathrm{Te}(\mathrm{I})-P n-\mathrm{Te}(\mathrm{II})-P n$-Te(I)] layers in (c) and (d) (Sb: Z=51, Te: Z=52, Bi: Z=83). Some examples are marked with the white arrows. 


\section{B. Solid solution of Bi atoms on Pn sites}

Fig. 2 shows measured and simulated STEM-HAADF results which are in good agreement. Further HAADF simulation and measurement data are presented at the supplementary material (Figs. S6 and S7). Bi atoms have a higher atomic number $Z=$ 83 and are thus nicely visible in HAADF by their brighter scattering contrast compared to $\mathrm{Sb}(Z=51)$ and $\mathrm{Te}(Z=52)$. Clearly, Bi occupies the $P n$ sites and is not visible at Te sites. Some contrast variations on $P n$ sites for the $x=0.24$ is according to the image simulations consistent with statistical occupation of $P n$ columns. The TEM lamellas in Fig. 2 have a thickness of about $15 \mathrm{~nm}$. For $x=0.24$, this results in an average number of $9 \mathrm{Bi}$ atoms per $35 \mathrm{Pn}$ atoms on a column. According to the image simulations, the contrast variation on the $P n$ columns in the experimental HAADF images is consistent with the standard deviation of $\pm 3 \mathrm{Bi}$ per column and thus proves the presence of a solid solution of $\mathrm{Bi}$ atoms on the Pn sites.

\section{Static point and thermal lattice disorder}

Selected area electron diffraction patterns reflect high crystallinity of the $\mathrm{Sb}_{2} \mathrm{Te}_{3}$ and $\mathrm{Bi}_{2} \mathrm{Te}_{3}$ films, where only the Bragg peaks of the films and the substrate are observed (Fig. S8). For the $\left(\mathrm{Sb}_{1-\mathrm{x}} \mathrm{Bi}_{\mathrm{x}}\right)_{2} \mathrm{Te}_{3}$ solid solutions electron diffraction for $x=0.12$ and $x=0.24$ show additional weak spots as well as diffuse streak like diffracted contrast along $(00 l)$. A detailed comparison of corresponding spots at $x=0$ and $x=0.24$ is shown in Fig. 3 (a) and (b), respectively. Both reflect the loss of long-range order along the (001) direction in the solid solutions. The weak additional spots are due to $\mathrm{Bi} / \mathrm{Sb}$ point disorder on Pn columns which can effectively increase the unit cell along the cdirection. Since $\mathrm{Sb}_{2} \mathrm{Te}_{3}$ and $\mathrm{Bi}_{2} \mathrm{Te}_{3}$ belong to the same space group, the $\mathrm{Sb}$ and $\mathrm{Bi}$ atoms share the same crystallographic sites in the lattice. The additional spots are consistent to the HRSTEM images with random distribution of $\mathrm{Bi}$ on Pn sites. In addition, the diffuse streak like diffracted contrast along (001) is consistent to thermal diffuse scattering (TDS) ${ }^{[39,40]}$. It points to thermal disorder of the c-axis lattice parameter, which is a result of strongly anharmonic bonding. This diffuse scattering is also visible in the compilation of the two intensity profiles presented at Fig. 3 (c), showing peak broadening and additional shoulders for $x=0.24$ (for $x=0.12$ see Fig. S8 (b)). 

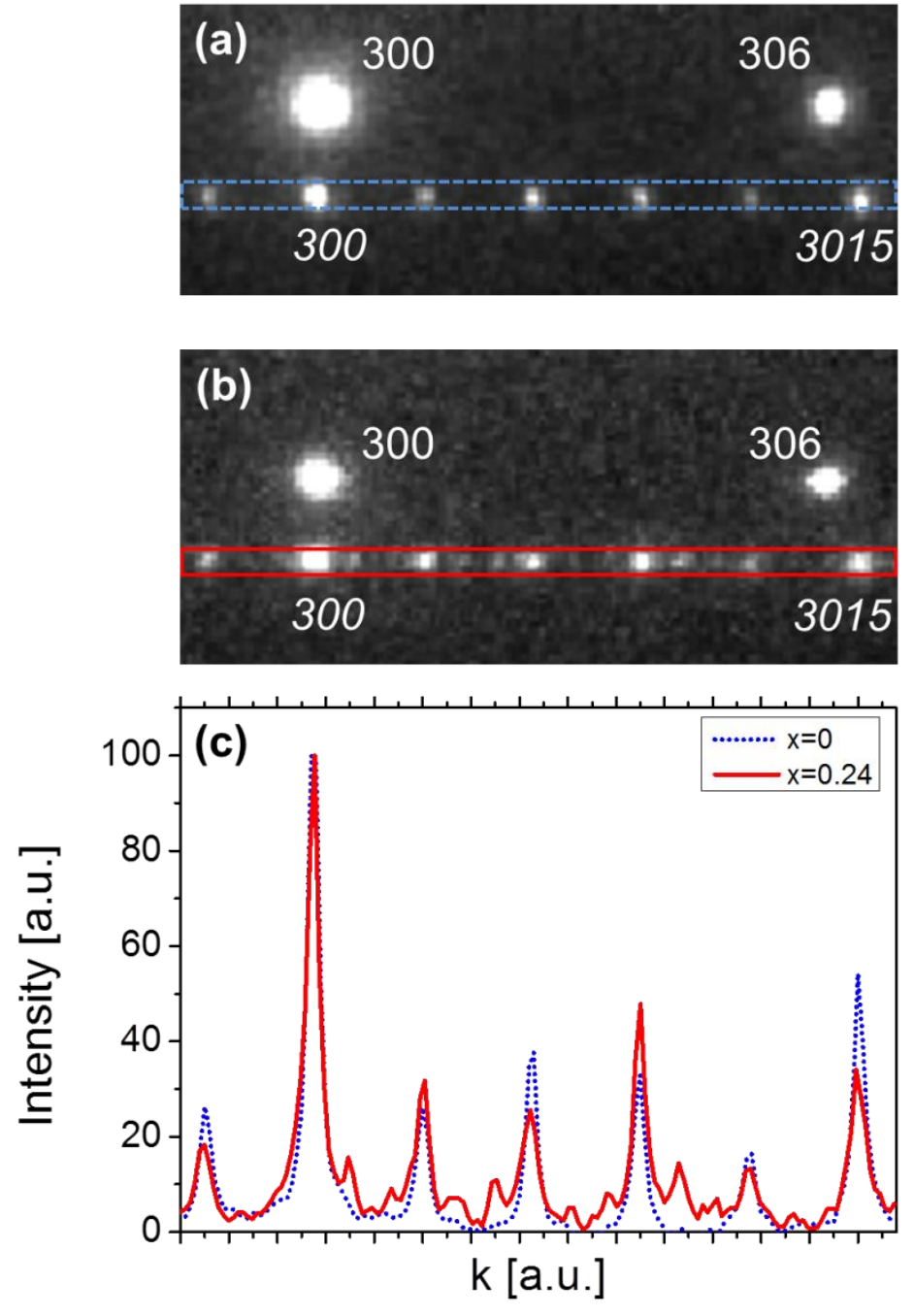

FIG. 3. Electron diffraction with dominant Bragg diffraction in the $\mathrm{Sb}_{2} \mathrm{Te}_{3}$ film (a) and a mixture of Bragg and diffuse scattering in the $\mathrm{Sb}_{0.76} \mathrm{Bi}_{0.24} \mathrm{Te}_{3}$ film (b). Line profiles are shown in (c). For $\mathrm{x}=0.24$, additional weak spots between the 300 and 3015 reflections indicate an enlargement of the unit cell in c-direction due to $\mathrm{Bi} / \mathrm{Sb}$ point disorder on $\mathrm{Pn}$ sites. In addition, the pronounced diffuse scattering streaks reflects thermal disorder in the c-lattice parameter. Italic indexing refers to spots of the film, whereas others are diffraction spots from the substrate. (a) and (b) are cutouts from SAED patterns shown in Fig. S8 (a) and S8 (c) in the supplementary material.

\section{Cross-plane lattice thermal conductivity}

Fig. 4 (a) shows the measured temperature dependence of the cross-plane thermal conductivity $\kappa_{c}(T)$ for all samples. In order to get access to the intrinsic lattice thermal conductivity $\kappa_{c, l}(T)$ of the films, first the contribution of insulation layers as well as the thermal boundary resistances (Kapitza resistances) were subtracted carefully (see ref. $^{[4]}$ ). After subtraction of the electronic contribution $\kappa_{c, e}(T)$ (see supplementary 
material), we obtain the lattice contribution to the thermal conductivity $\kappa_{c, l}(T)$, shown in Fig. 4 (b).
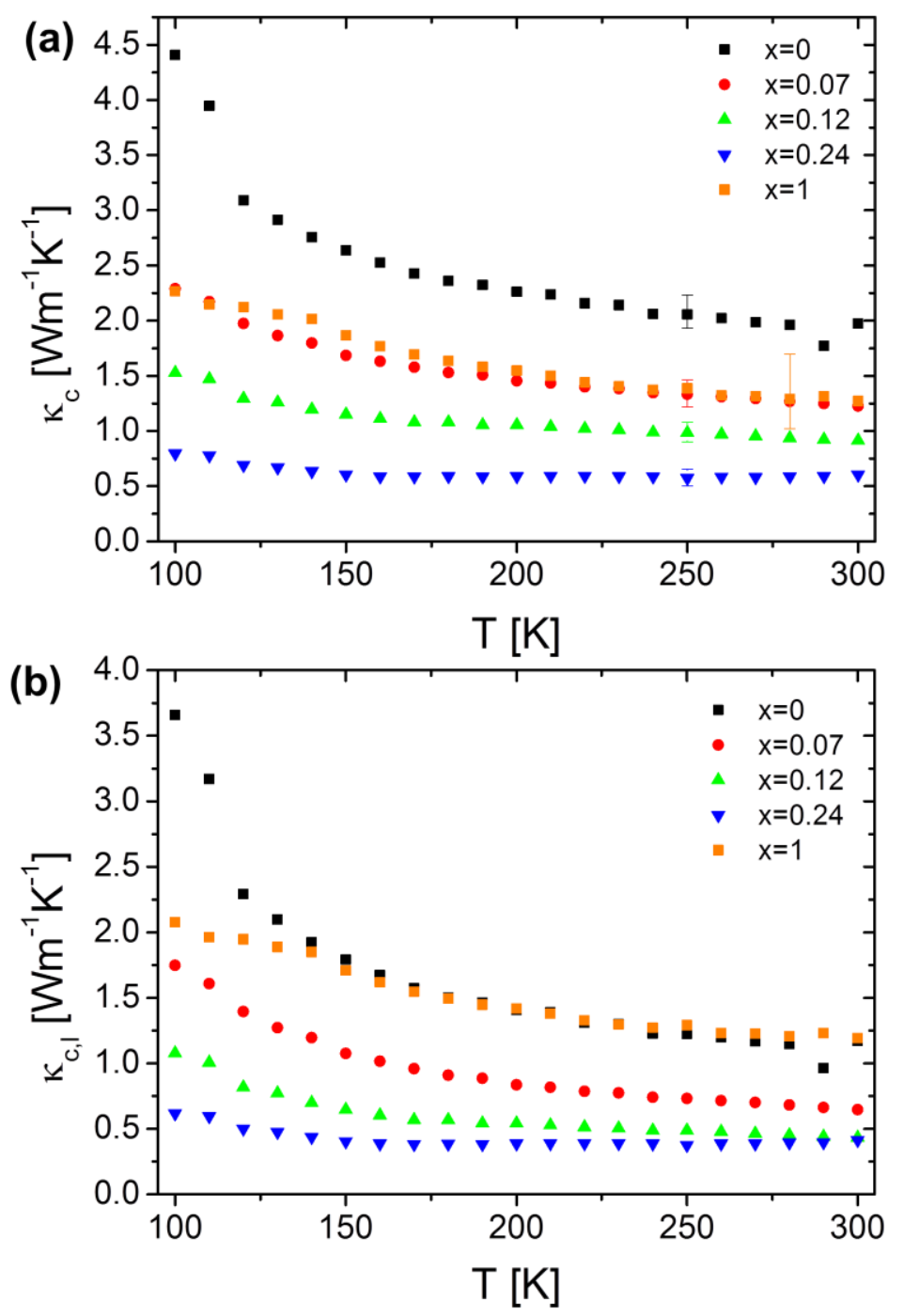

FIG. 4. Thermal conductivity of epitaxial $\left(\mathrm{Sb}_{1-\mathrm{x}} \mathrm{Bi}_{\mathrm{x}}\right)_{2} \mathrm{Te}_{3}$ films along the c-axis, obtained from $3 \omega$ measurements. (a) Total thermal conductivity $\kappa_{c}(T)$. (b) Lattice thermal conductivity $\kappa_{c, l}(T)$ after subtraction of the electronic contribution $\kappa_{c, e}(T)$.

Fig. 5 (a) shows the experimental $\kappa_{c, l}(T)$ data (open symbols) in combination with fits of eq. (1) in the experimental section (lines in the respective color) for all doping. The minimum lattice thermal conductivity along the $\mathrm{c}$-axis for $\mathrm{Sb}_{2} \mathrm{Te}_{3}$, relevant for $x=0.24$, is also shown and discussed later. Fig. 5 (b) and (c) show the obtained values of the fit parameters $A^{-1}$ for phonon-phonon scattering and $B^{-1}$ for Rayleigh scattering (eqs. (6) and (7), respectively in the experimental section). $A^{-1}$ and $B^{-1}$ in Fig. 5 (b) and (c) are proportional to the doping dependent scattering rates due to Umklapp- and Rayleigh scattering, respectively. 

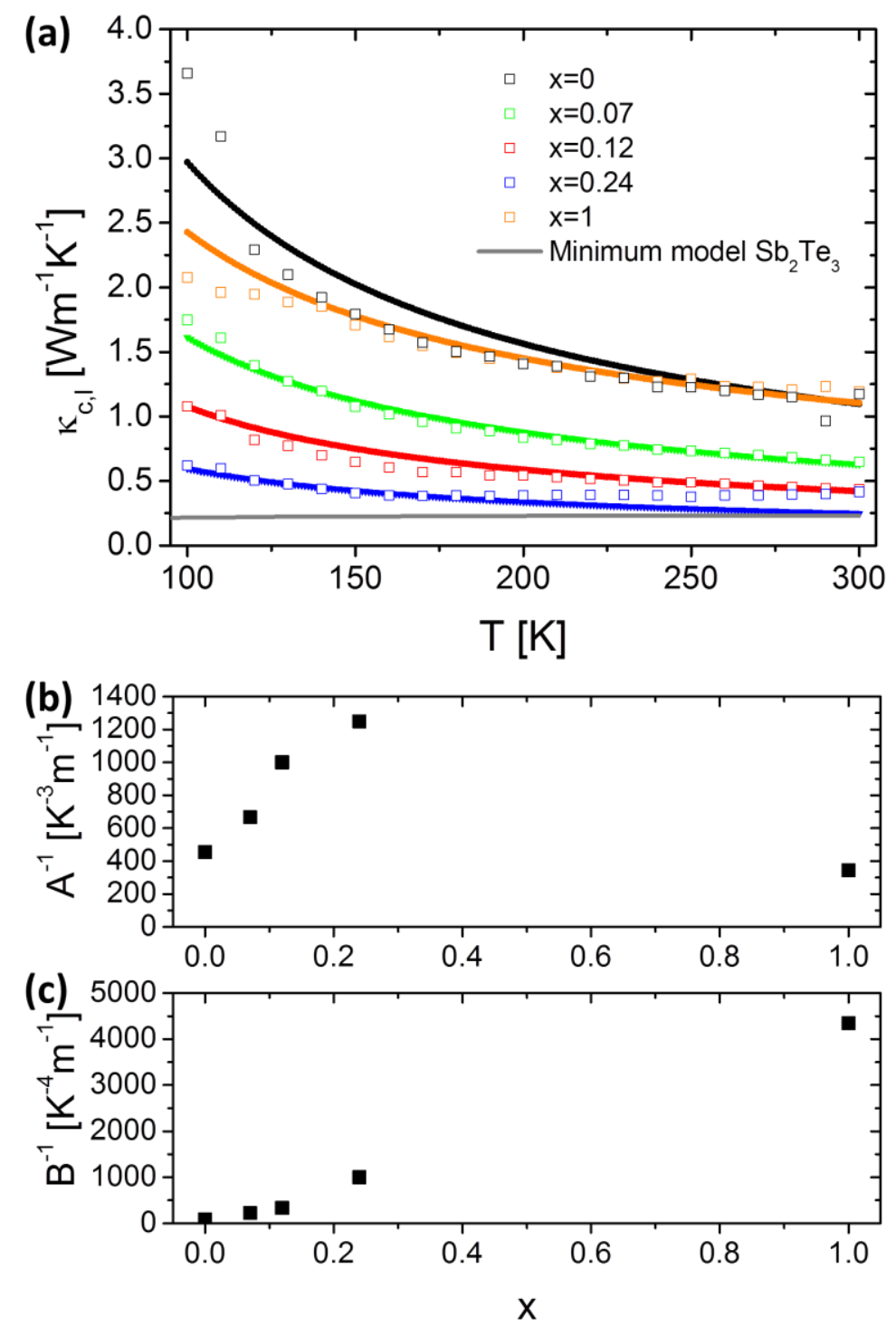

FIG. 5. (a) Comparison of experimental and theoretical temperature dependence of $\kappa_{c, l}(T)$, including the calculated minimum lattice thermal conductivity of $\mathrm{Sb}_{2} \mathrm{Te}_{3}$ in c-direction. The two plots below show the obtained fit parameters for Umklapp scattering $A^{-1}$ (b) in eq. (6) and Rayleigh scattering $B^{-1}$ (c) in eq. (7) vs. doping $x$. They are a measure of the scattering rates.

\section{E. Relevant phonon frequencies}

Fig. 6 shows the theoretical lattice thermal conductivity as a function of the upper integration boundary of the phonon angular frequency at $T=100,200$ and $300 \mathrm{~K}$ for two doping levels, $x=0$ and $x=0.24$ (eqs. (S1) and (S2) in the supplementary material). For other doping levels and for the underlying differential thermal conductivity, see Fig. S12 - S16.

The comparison of Figs. 6 (a) and (b) shows that for the highly Bi doped sample at $x=0.24$ a higher percentage of the whole $\kappa_{c, l}$ is carried by lower frequency phonons 
compared to $x=0$. For example, at $300 \mathrm{~K}, 90 \%$ of the $\kappa_{c, l}$ is carried by phonons up to $\omega$ $=6600 \mathrm{GHz}$ and $4600 \mathrm{GHz}$ at $x=0.0$ and 0.24 , respectively. Since the bandgap between $\mathrm{A}$ and $\mathrm{O}$ modes in $\mathrm{Sb}_{2} \mathrm{Te}_{3}$ is between $7700 \mathrm{GHz}$ and $8200 \mathrm{GHz}$ in cdirection $^{[23]}$, the contribution of the $\mathrm{O}$ modes as well as of a the higher frequency $\mathrm{A}$ modes to $\kappa_{c, l}$ are suppressed for $x=0.24$ compared to $x=0$. Overall one can say that optical phonons only play a minor role in heat transport and do not contribute to thermal conductivity in the solid solutions.
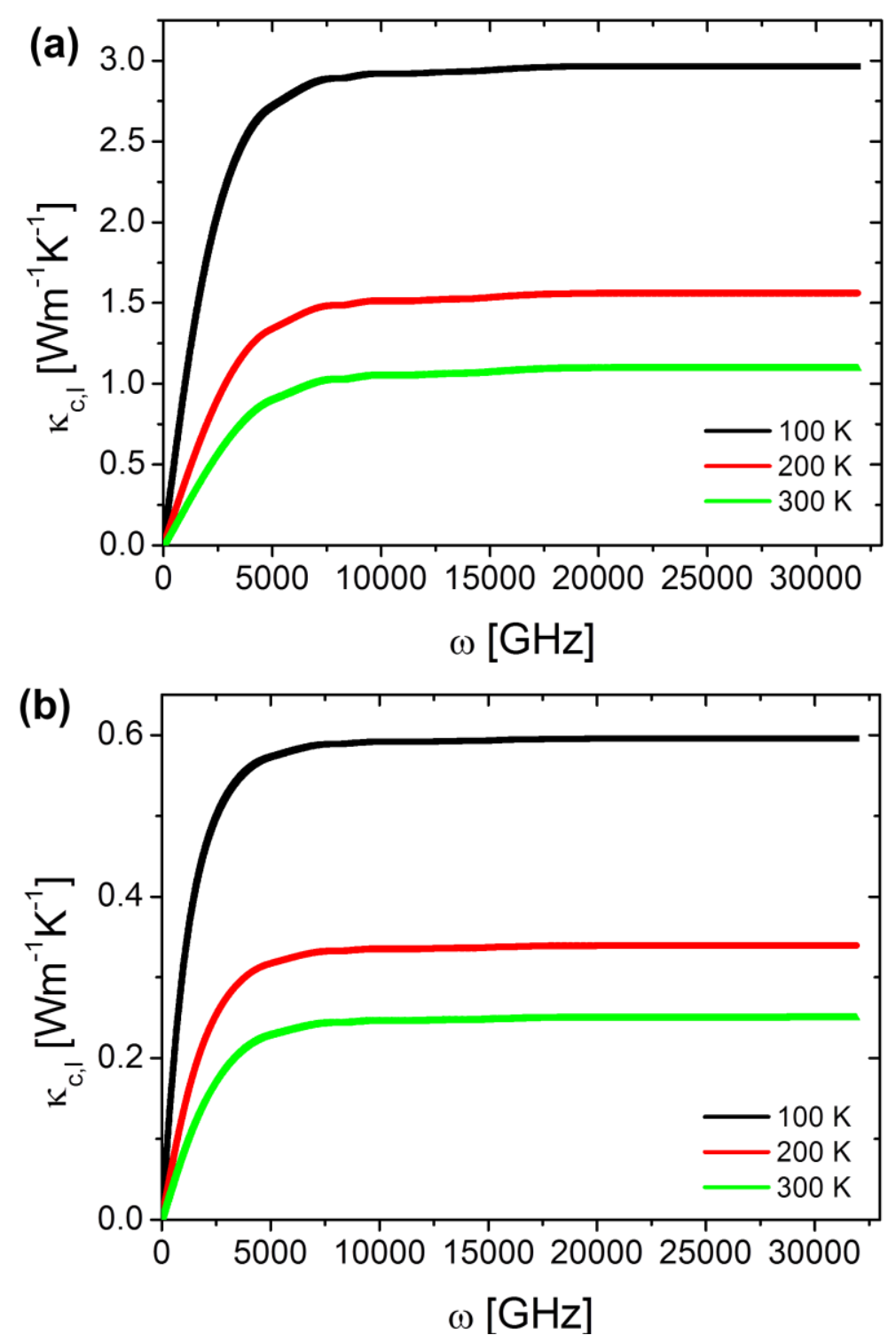

FIG. 6. Lattice thermal conductivity as a function of the upper integration boundary $\omega$ at $T=$ 100,200 and $300 \mathrm{~K}$, for (a) $x=0$ and (b) $x=0.24$. The transition to saturation gives the upper frequency limit of the phonons contributing to thermal conductivity. 


\section{F. Phonon glass state for $x=0.24$}

The obtained temperature characteristics of $\kappa_{c, l}(T)$ for the epitaxial film with $x=0.24$ deviates from those of the other samples. As shown in Fig. 7, for low temperatures up to $T=170 \mathrm{~K}$ the phonon model based on eq. (1) still is well applicable. This is presumably due to the low frequency acoustic phonons which dominate $\kappa_{c, l}$ in the low temperature range. At higher temperatures, a plateau respectively a very moderate increase in $\kappa_{c, l}(T)$ is observed, that cannot be explained in the phonon picture. It is in contrast to the expected further decrease of $\kappa_{c, l}(T)$ due to an increase of the Umklapp scattering rate with increasing temperature and related phonon population. Consequently, the lattice thermal conductivity of the sample above $T=150 \mathrm{~K}$ resembles that one of an amorphous solid. Using the minimum lattice thermal conductivity model, i.e. eq. $(8)^{[28]}$, based on the assumption of $l_{m f p}=\frac{\lambda}{2}$, our calculations reveal a temperature independent $\kappa_{c, l}(T)$.We use literature data for the average acoustic phonon velocities of $\mathrm{Bi}_{2} \mathrm{Te}_{3}{ }^{[18]}$ and $\mathrm{Sb}_{2} \mathrm{Te}_{3}{ }^{[23]}$ as well as for the lattice parameters of $\mathrm{Sb}_{2} \mathrm{Te}_{3}{ }^{[41]}$ respectively $\mathrm{Bi}_{2} \mathrm{Te}_{3}{ }^{[42]}$. The minimum thermal conductivity of $\mathrm{Bi}_{2} \mathrm{Te}_{3}$ is below that of $\mathrm{Sb}_{2} \mathrm{Te}_{3}$, due to the higher mass of $\mathrm{Bi}$ compared to $\mathrm{Sb}$. Both curves are below the experimental curve for the $x=0.24$ film. This can be either due to the used approximations within the minimum thermal conductivity model. It can predict absolute values of $\kappa_{c, l}(T)$ only with limited accuracy as reviewed by Cahill et al. ${ }^{[28]}$. Furthermore, as indicated by the mean free path in Fig. 8, the possible transition to the phonon glass state might affect only higher frequency modes, whereas $l_{m f p}$ of lower frequency acoustic phonons remain above the $\frac{\lambda}{2}$ threshold. The latter idea is supported by the transition of the temperature dependence of $\kappa_{c, l}(T)$ form the phonon scattering to the phonon glass regime at a temperature close to the Debye temperature. 


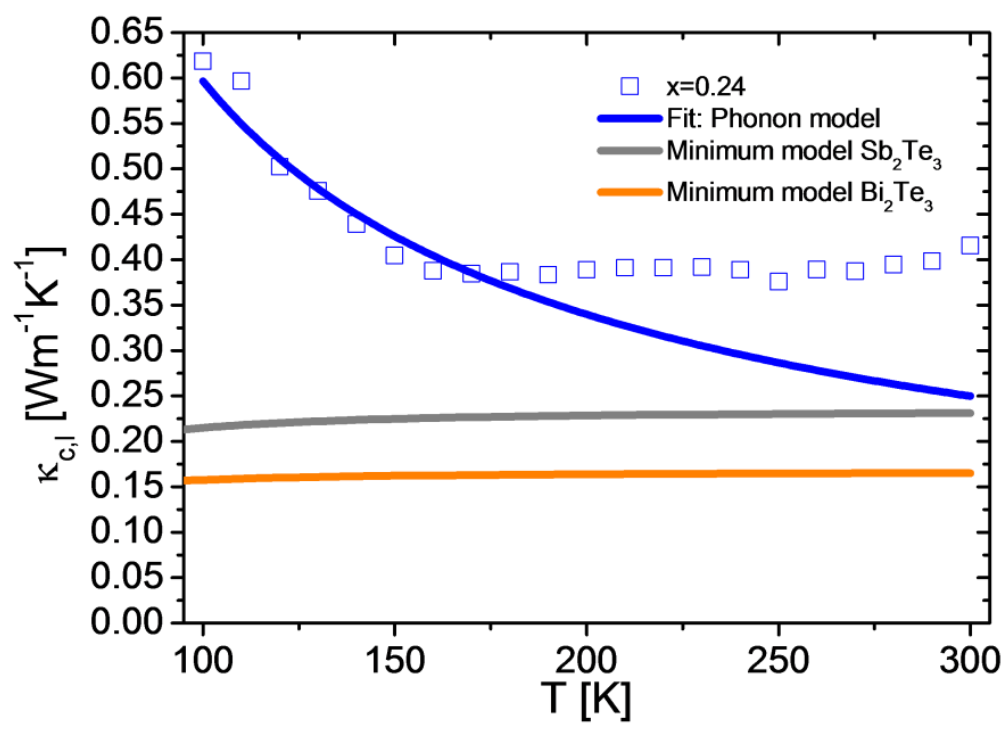

FIG. 7. Experimental lattice thermal conductivity of the epitaxial film with Bi content $x=0.24$. Blue: Calculated curve within the Callaway model, fitted to the low temperature values of $\kappa_{c, l}(T)$. Grey $\left(\mathrm{Sb}_{2} \mathrm{Te}_{3}\right)$ and orange $\left(\mathrm{Bi}_{2} \mathrm{Te}_{3}\right)$ : Minimum thermal conductivity model for disordered systems with $l_{m f p}=\frac{\lambda}{2}$.

\section{G. Phonon mean free path}

Fig. 8 shows the phonon mean free path versus the angular frequency for all samples. It decreases with increasing $\mathrm{Bi}$ content due to increased scattering. The plotted half phonon wavelength gives a rough estimate of the applicability range of the phonon model. For $x=0.12$ at $T=300 \mathrm{~K}$, this is $\omega=4100 \mathrm{GHz}$. Thus, higher frequency A modes can contribute to the c-axis phonon transport. In contrast, for $x=0.24$, the application limit of the notion of a phonon mean free path shifts down to $\omega=3000 \mathrm{GHz}$. Consequently, the higher frequency A modes seems to lie in the glass regime. For a detailed analysis of the contribution of Umklapp $l_{m f p, U}$ respectively Rayleigh scattering $l_{m f p, R}$ to the total $l_{m f p}$ see Figs. S17 and S18, showing the dominance of Umklapp scattering over Rayleigh scattering in the frequency range relevant for thermal transport. 

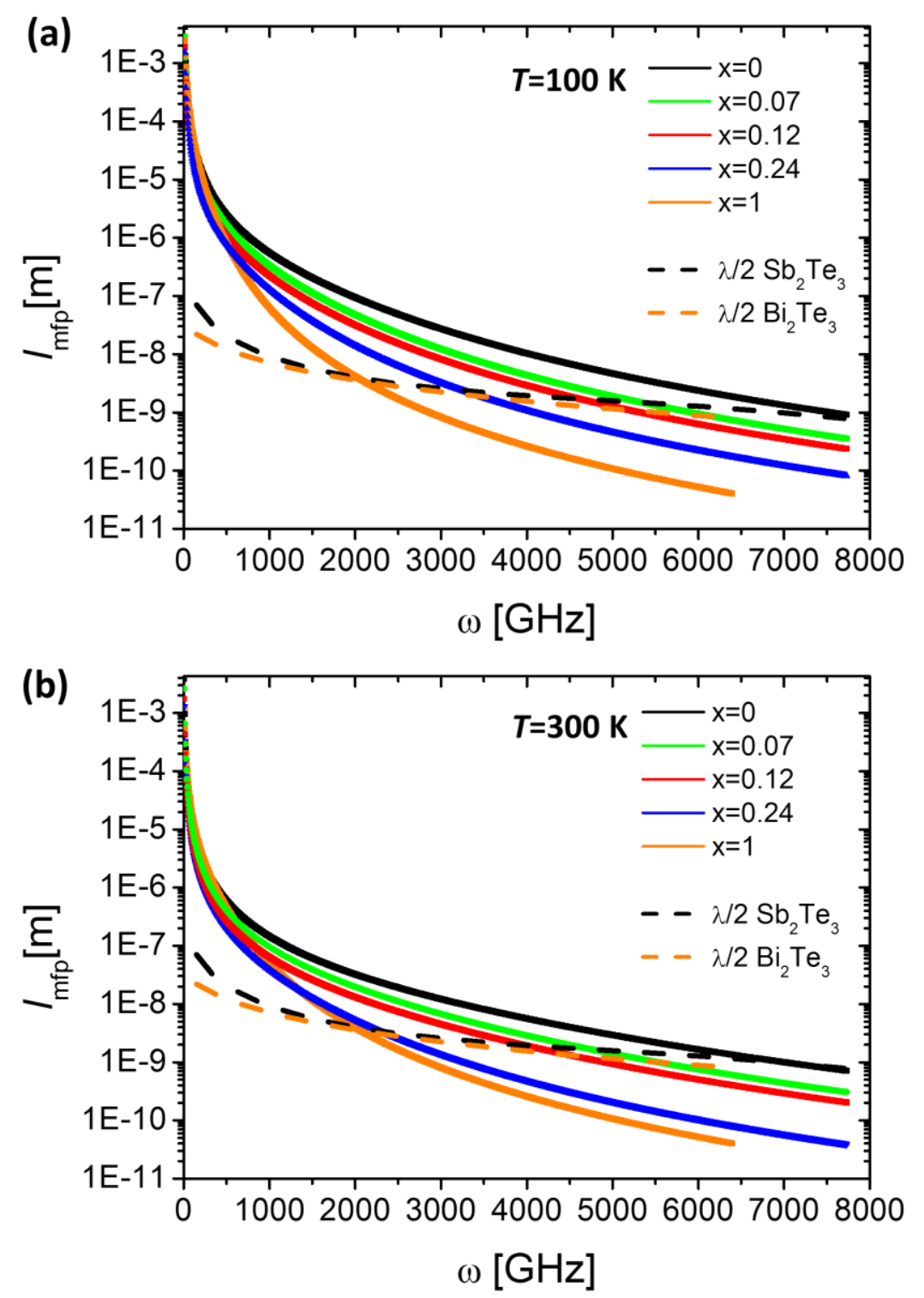

FIG. 8. Phonon mean free path $l_{m f p}$ versus the angular frequency for (a) $100 \mathrm{~K}$ and (b) 300 $\mathrm{K}$, as calculated from eqs. (5), (6) and (7) and using the parameters $A^{-1}$ and $B^{-1}$ in Fig. 5. Additionally, the half phonon wavelength is plotted. At frequencies where $l_{m f p} \leq \frac{1}{2} \lambda$ the approximation of phonon quasiparticles as eigenstates of a harmonic periodic lattice break down.

Note that in Fig. 8 for $\mathrm{Bi}_{2} \mathrm{Te}_{3}$ the obtained $l_{m f p}(\omega, T=300 \mathrm{~K})$ at $\omega \geq 1100 \mathrm{GHz}$, is even lower than that of the solid solutions which is linked to the higher density of states at low frequencies (compare ref. ${ }^{[23]}$ for $\mathrm{Sb}_{2} \mathrm{Te}_{3}$ and ref. ${ }^{[18]}$ for $\mathrm{Bi}_{2} \mathrm{Te}_{3}$ ). Furthermore, the relatively high parameter $B^{-1}$ for $\mathrm{Bi}_{2} \mathrm{Te}_{3}$ indicates a higher density of point defects. 


\section{DISCUSSION}

We first want to focus on the pure material systems $\mathrm{Sb}_{2} \mathrm{Te}_{3}$ and $\mathrm{Bi}_{2} \mathrm{Te}_{3}$ : The Umklapp scattering parameter $A^{-1}$ is similar for both materials, being slightly higher in $\mathrm{Sb}_{2} \mathrm{Te}_{3}$ compared to $\mathrm{Bi}_{2} \mathrm{Te}_{3}$. The most significant change is the higher Rayleigh scattering reflected in a high parameter $B^{-1}$. This might reflect a higher preparation related defect density in the investigated film, due to different growth conditions.

Our results on the doping dependence of the scattering parameters $B^{-1}$ and $A^{-1}$ for Rayleigh and Umklapp scattering, respectively, yield an increasing point defect and phonon-phonon scattering with Bi doping $x$. At $x=0.24$ the lattice thermal conductivity shows a transition to a temperature independent behavior for $T>150 \mathrm{~K}$, indicating that higher frequency optical and acoustic phonon modes may reach the phonon glass limit.

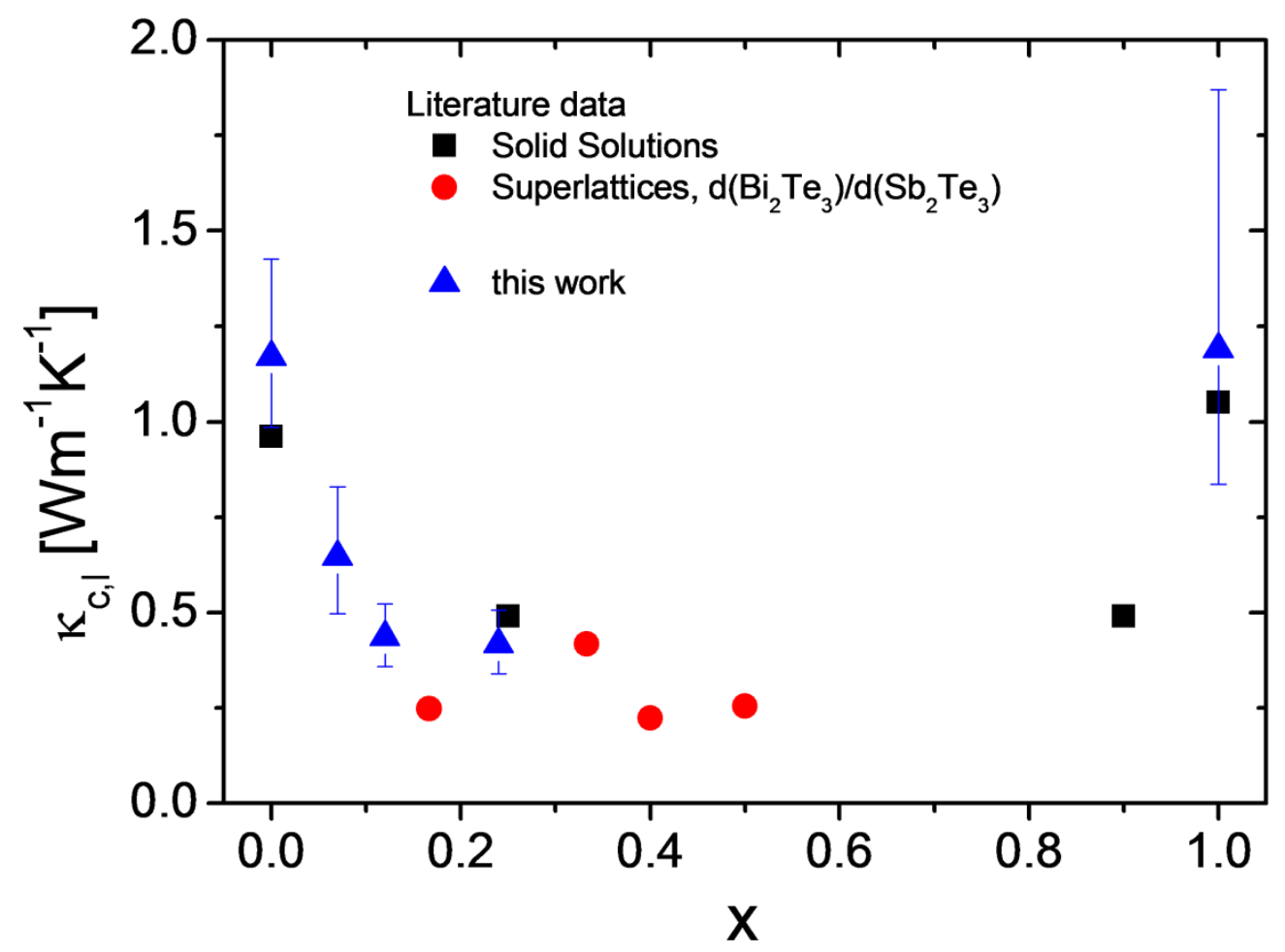

FIG. 9. Comparison of the lattice thermal conductivity in c-direction at $T=300 \mathrm{~K}$ with literature values for solid solutions ${ }^{[10,11]}$ and for a $\mathrm{Sb}_{2} \mathrm{Te}_{3} / \mathrm{Bi}_{2} \mathrm{Te}_{3}$ superlattices of different bilayer and single layer thicknesses (10/50 ̊; 30/60 ̊; 20/30 ̊; 30/30 ̊) as published by Venkatasubramanian ${ }^{[11]}$. 
Fig. 9 shows the comparison of our data with literature data at $300 \mathrm{~K}^{[10,11]}$, obtained by $3 \omega$ measurements of MOCVD-grown thin films. At $x=0$ and $x=1$ our $\kappa_{c, l}$ values are slightly higher, but close to the literature values within error bars. Note, that the measurement error of the literature data is not published. The authors state that they subtracted the thermal conductivity of the used insulation layer. However, heat spread effects enhanced by anisotropy and Kapitza resistances were not considered. Especially for materials with higher $\kappa_{c}$ (here: $x=0$ and $x=1$ ), the neglect of the Kapitza resistances results in too low values for $\kappa_{c}$. For the doped samples, our results are in good agreement with the solid solution at $x=0.25^{[10]}$ as well as with the published data of a superlattice with bilayer thickness of $0.9 \mathrm{~nm}\left(0.3 \mathrm{~nm} \mathrm{Bi}_{2} \mathrm{Te}_{3} / 0.6 \mathrm{~nm} \mathrm{Sb} \mathrm{Te}_{3}\right)^{[11]}$.

The published values for $\kappa_{c, l}$ of SLs with bilayer thicknesses of 0.5 and $0.6 \mathrm{~nm}$ are extrapolated from measurements at superlattices to a vanishing carrier density. With one exception, they are slightly lower ${ }^{[11]}$ compared to our values for the solid solutions. The difference between alloy and superlattice thermal conductivity has been interpreted as due to phonon blocking effects at the interfaces between the single layers. However, several publications reveal that the growth of superlattices with chemically sharp interfaces is inhibited by the required high substrate temperature for epitaxial growth: For sputtered SLs ${ }^{[12]}$, grain growth actually has been observed at 150 ${ }^{\circ} \mathrm{C}$ and for SLs synthesized by $\mathrm{MBE}^{[13]}$, TEM and XRD data showed that interdiffusion has been taken place at low temperatures of around $200{ }^{\circ} \mathrm{C}$. According to ref. ${ }^{[43]}$ the samples are synthesized by a MOCVD process with substrate temperatures of around $225{ }^{\circ} \mathrm{C}$ which may be already too high in case of $\mathrm{Bi}_{2} \mathrm{Te}_{3} / \mathrm{Sb}_{2} \mathrm{Te}_{3}$ SLs: The TEM data presented by ref. ${ }^{[4]}$ cannot exclude that interdiffusion already has happened during sample preparation.

Our results establish that low $\kappa_{c, l}(T) \approx 0.4 \mathrm{Wm}^{-1} \mathrm{~K}^{-1}$ can be reached without elaborate nanostructuring methods at $x=0.24$. For this doping level, a low and almost constant value of $\kappa_{c, l}(T) \approx 0.39-0.42 \mathrm{Wm}^{-1} \mathrm{~K}^{-1}$ is obtained in the temperature range from 160 to $300 \mathrm{~K}$. The low lattice thermal conductivity is found to be an intrinsic material property of the solid solution. Taking into account the solid solution literature data shown in Fig. 9, an almost doping independent and very low value at room temperature seems to be present in a large doping range. Point disorder due to statistical occupation of $P n$ sites by $\mathrm{Bi}$ doping increases the Rayleigh scattering as visible from the doping dependence of $B^{-1}(x)$ in Fig. 5 (c). More remarkably, it also further increases the Umklapp scattering $A^{-1}(x)$ in Fig. 5 (b). From the electron diffraction, evidence for the increase of thermal disorder of the c-lattice parameter with doping is consistent with the increase in phonon-phonon scattering and thus to an increase of the anharmonicity. We cannot separate out, whether this leads to a softening of specific phonon modes or to an amplification of selected phonon scattering channels due to disorder in strongly anharmonic potentials as suggested in ref. $^{[44]}$. 


\section{SUMMARY AND CONCLUSION}

In summary, we have performed detailed temperature dependent measurements of the $\mathrm{c}$-axis thermal conductivity in epitaxial, nearly single crystalline $\left(\mathrm{Sb}_{1-\mathrm{x}} \mathrm{Bi}_{\mathrm{x}}\right)_{2} \mathrm{Te}_{3}$ films using the $3 \omega$ method. The contribution of Kapitza resistances and the thermal resistance of the used insulation layer were carefully measured and subtracted in order to get the intrinsic $\kappa_{c}(T)$. Subsequently, we subtracted the electronic contribution by means of literature data receiving the lattice contribution $\kappa_{c, l}(T)$. Theoretical calculations of the c-axis lattice thermal conductivity were performed based on literature data of the density of states and the phonon dispersions in $\mathrm{Sb}_{2} \mathrm{Te}_{3}$ and $\mathrm{Bi}_{2} \mathrm{Te}_{3}$. Based on HRTEM results of every studied film, the density of 2D and 3D defects has been found to be negligible small: Their average distance compares to the $l_{m f p}(\omega)$ only at very low frequencies of $\omega<1200 \mathrm{GHz}$ which do not significantly contribute to $\kappa_{c, l}(T)$. Consequently, Umklapp and Rayleigh scattering have been identified as the dominant scattering mechanisms, where Umklapp scattering dominates the mean free path in the frequency range relevant for thermal conductivity at room temperature. Remarkably, we obtain both an increase of phonon-phonon scattering as well as of Rayleigh scattering with an increasing substitution of $\mathrm{Sb}$ atoms by $\mathrm{Bi}$ atoms. The Umklapp scattering due to the large anharmonicity of $\mathrm{Sb}_{2} \mathrm{Te}_{3}$ is slightly higher compared to that of the $\mathrm{Bi}_{2} \mathrm{Te}_{3}$ film, which is in good agreement with the published Grüneisen parameters ${ }^{[21]}$. Furthermore, at $x=0.24$ a transition of $\kappa_{c, l}(T)$ to a phonon glass is observed by the anomalous temperature dependence. Based on our results we conclude that the very low lattice thermal conductivities in the solid solution $\left(\mathrm{Sb}_{1-}\right.$ $\left.{ }_{x} \mathrm{Bi}_{\mathrm{x}}\right)_{2} \mathrm{Te}_{3}$ are intrinsic, caused by the combination of low phonon propagation velocities and small mean free paths. The origin of the latter is mostly the relatively strong anharmonicity further enhanced by Rayleigh scattering.

Therefore, it seems to be justified by our measurements to use the term "Phonon glass - electron crystal" for the higher doping levels: The lattice conductivity is comparable low to a glass, but the sample shows high crystallinity. At $x=0.25$ the specific resistance $\rho$ along c-axis is between $2.9-4.6 \mathrm{~m} \Omega \mathrm{cm}$ at room temperature ${ }^{[45]}$. This is one order of magnitude below highly doped Si. In addition, polycrystalline material with $x=0.25$ shows a similar specific resistance of $\rho=2.5 \mathrm{~m} \Omega \mathrm{cm}$ at room temperature ${ }^{[7]}$, whereas despite the presence of grain boundaries, the thermal conductivity is with 0.8 $\mathrm{Wm}^{-1} \mathrm{~K}^{-1}$ slightly above the total $\kappa_{c}$ value of $0.6 \mathrm{Wm}^{-1} \mathrm{~K}^{-1}$ in our film. The combination of low thermal conductivity and high electric conductivity is the origin of good thermoelectric performance around that composition. Such a "phonon glass - electron crystal" state can thus be reached in the solid solutions without any nanostructuring methods and our results give rise to further research on doping series in vdW layered thermoelectric materials. 


\section{SUPPLEMENTARY MATERIAL}

See supplementary material document for tables $\mathrm{S} 1-\mathrm{S} 3$ and figures $\mathrm{S} 1-\mathrm{S} 18$.

\section{ACKNOWLEDGEMENT}

Financial support by the Deutsche Forschungsgemeinschaft DFG (S. Schulz, project SCHU 1069/20-1; C. Jooss, project JO 348/12-1) as well as by SFB 1073 is gratefully acknowledged.

\section{References}

[1] C. Uher, ed. Materials aspect of thermoelectricity, Taylor \& Francis 2017.

[2] H. J. Goldsmid and R. W. Douglas, Br. J. Appl. Phys. 5, 386 (1954).

[3] M. Z. Hasan and C. L. Kane, Rev. Mod Phys. 82, 3045 (2010).

[4] F. Rieger, K. Kaiser, G. Bendt, V. Roddatis, P. Thiessen, S. Schulz, and C. Jooss, J. Appl. Phys. 123, 175108 (2018).

[5] C. V. Manzano, B. Abad, M. Muñoz Rojo, Y. R. Koh, S. L. Hodson, A. M. Lopez Martinez, X. Xu, A. Shakouri, T. D. Sands, T. Borca-Tasciuc, and M. Martin-Gonzalez, Sci Rep. 6, 19129 (2016).

[6] C. Zhang, M. de la Mata, Z. Li, F. J. Belarre, J. Arbiol,K. A. Khor, D. Poletti, B. Zhu, Q. Yan, and Q. Xiong, Nano Energy 15, 688 (2015).

[7] F. Serrano-Sánchez, M. Gharsallah, N. M. Nemes, N. Biskup, M. Varela, J. L. Martínez, M. T. Fernández-Díaz, and J. A. Alonso, Sci. Rep. 7, 6277 (2017).

[8] J. R. Drabble and C. H. L. Goodman, J. Phys. Chem. Solids 5, 142 (1958).

[9] D. Lencer, M. Salinga, B. Grabowski, T. Hickel, J. Neugebauer, and M. Wuttig, Nat. Mater. 7, 972 (2008).

[10] R. Venkatasubramanian, E. Siivola, T. Colpitts, and B. O'Quinn, Nature 413, 597 (2001).

[11] R. Venkatasubramanian, Phys. Rev. B 61, 3091 (2000).

[12] M. Winkler, X. Liu, A.-L. Hansen, J. D. König, W. Bensch, L. Kienle, H. Böttner, and K. Bartholomé, Nanothermoelectrics 1, 1 (2013).

[13] A.-L. Hansen, T. Dankwort, M. Winkler, J. Ditto, D. C. Johnson, J. D. Koenig, K. Bartholomé, L. Kienle, and W. Bensch, Chem. Mater. 26, 6518 (2014).

[14] For a recent review article see: T. Dankwort, A.-L. Hansen, M. Winkler, U. Schürmann, J. D. Koenig, D. C. Johnson, N. F. Hinsche, P. Zahn, I. Mertig, W. Bensch, and L. Kienle., Phys. Status Solidi A 213, 662 (2016).

[15] M. Winkler, X. Liu, U. Schürmann, J. D. König, L. Kienle, W. Bensch, and H. Böttner, Z. Anorg. Allg. Chem. 638, 2441 (2012).

[16] D. Das, S. Das, P. Singha, K. Malik, A. K. Deb, A. Bhattacharyya, V. A. Kulbachinskii, R. Basu, S. Dhara, S. Bandyopadhyay, and A. Banerjee, Phys. Rev. B 96, 064116 (2017).

[17] Y. Tian, S. Jia, R. J. Cava, R. Zhong, J. Schneeloch, G. Gu, and K. S. Burch, Phys. Rev. B 95, 094104 (2017).

[18] O. Hellman and D. A. Broido, Phys. Rev. B 90, 134309 (2014).

[19] C. K. Gan and C. H. Lee, Comput. Mater. Sci. 151, 49 (2018).

[20] K. H. Park, M. Mohamed, Z. Aksamija, and U. Ravaioli, J. Appl. Phys. 117, 015103 (2015).

[21] D. Bessas, I. Sergueev, H.-C. Wille, J. Perßon, D. Ebling, and R. P. Hermann, Phys. Rev. B 86, 224301 (2012).

[22] S. Lee, K. Esfarjani, T. Luo, J. Zhou, Z. Tian, and G. Chen, Nat. Commun. 5, 3525 (2014).

[23] B.-T. Wang, P. Souvatzis, O. Eriksson, and P. Zhang, J. Chem. Phys. 142, 174702 (2015).

[24] N.-W. Park, W.-Y. Lee, J.-E. Hong, T.-H. Park, S.-G. Yoon, H. Im, H. S. Kim, and S.-K. Lee, Nanoscale Res. Lett. 10, 20 (2015). 
[25] P. Lošt'ák, Č. Drašar, A. Krejčová, L. Beneš, J. S. Dyck, W. Chen, and C. Uher, J. Cryst. Growth 222, 565 (2001).

[26] P. Lošt'ák, Č. Drašar, J. Horàk, Z. Zhou, J. S. Dyck, and C. Uher, J. Phys. Chem. Sol. 67, 1457 (2006).

[27] J. Callaway, Phys. Rev. 113, 1046 (1959).

[28] D. G. Cahill, S. K. Watson, and R. O. Pohl, Phys. Rev. B 46, 6131 (1992).

[29] G. A. Slack, Solid State Phys. 34, 1 (1979).

[30] G. Bendt, K. Kaiser, A. Heckel, F. Rieger, D. Oing, A. Lorke, N. Perez Rodriguez, G. Schierning, C. Jooss, and S. Schulz, Semicond. Sci. Technol. 33, 105002 (2018).

[31] C. Koch, PhD dissertation, Arizona State University 2002.

[32] A. Jacquot, N. Farag, M. Jaegle, M. Bobeth, J. Schmidt, D. Ebling, and H. Böttner, J. Electron. Mater. 39, 1861 (2010).

[33] H. J. Goldsmid, Proc. Phys. Soc. B 69, 203 (1956).

[34] H. Rauh, R. Geick, H. Köhler, N. Nücker, and N. Lehner, J. Phys. C: Solid State Phys. 14, 2705 (1981).

[35]X. X. Yang, Z. F. Zhou, Y. Wang, R. Jiang, W. T. Zhen et al., J. Appl. Phys. 112, 083508 (2012).

[36] J. O. Jenkins, J. A. Rayne, and R. W. Ure, Jr., Phys. Rev. B 5, 3171 (1972).

[37] M. de Jong, W. Chen, T. Angsten, A. Jain, R. Notestine, A. Gamst, M. Sluiter, C. Krishna Ande, S. van der Zwaag, J. J. Plata, C. Toher, S. Curtarolo, G. Ceder, K. A. Persson, and M. Asta, Scientific Data 2, 150009 (2015).

[38] W. M. Haynes, CRC Handbook of Chemistry and Physics, CRC Press, Boca Raton, FL, 97th edition 2016.

[39] C. R. Hall and P. B. Hirsch, Proc. Royal Soc. A 286, 158 (1965).

[40] Z. L. Wang, Philos. Mag. B 65, 559 (1992).

[41] W.-S. Kim, J. Alloys Compd. 252, 166 (1997).

[42] Y. Feutelais, B. Legendre, N. Rodier, and V. Agafonov, Mater. Res. Bull. 28, 591 (1993).

[43] R. Venkatasubramanian, T. Colpitts, B. O'Quinn, S. Liu, N. El-Masry, and M. Lamvik, Appl. Phys. Lett. 75, 1104 (1999).

[44] P. Erhart, P. Hyldgaard, and D. O. Lindroth, Chem. Mater. 27, 5511 (2015).

[45] H. Scherrer und S. Scherrer, CRC Handbook of Thermoelectrics, ed. By D. M. Rowe, CRC Press, Chap. 19, p. 221 (1995). 

Supplementary material to:

\section{Transition into a phonon glass in crystalline thermoelectric $\left(\mathrm{Sb}_{1-\mathrm{x}} \mathrm{Bi}_{\mathrm{x}}\right)_{2} \mathrm{Te}_{3}$ films}

F. Rieger ${ }^{1}$, V. Roddatis ${ }^{1}$, K. Kaiser ${ }^{2}$, G. Bendt ${ }^{2}$, S. Schulz ${ }^{2}$, and C. Jooss ${ }^{1}$

${ }^{1}$ Institute of Material Physics, University of Göttingen, 37077 Göttingen, Germany

${ }^{2}$ Institute of Inorganic Chemistry and Center for Nanointegration Duisburg-Essen

(Cenide), University of Duisburg-Essen, 45141 Essen, Germany

\section{Preparation conditions and sample compositions:}

Details about the deposition parameters, thickness and composition of the investigated samples are presented in Tables S1 and S2.

\begin{tabular}{|l|l|l|l|l|l|}
\hline Bi content $x$ & $\begin{array}{l}\text { Substrate } \\
\text { temperature }\left[{ }^{\circ} \mathrm{C}\right]\end{array}$ & $\begin{array}{l}\text { Evaporator } \\
\text { temperature }\left[{ }^{\circ} \mathrm{C}\right]\end{array}$ & $\begin{array}{l}\text { Pressure } \\
{[\mathrm{mbar}]}\end{array}$ & $\begin{array}{l}\text { Deposition } \\
\text { time }[\mathrm{min}]\end{array}$ & $\begin{array}{l}\text { Thickness } \\
{[\mathrm{nm}]}\end{array}$ \\
\hline 0 & 333 & 600 & 20 & 40 & 1184 \\
\hline 0.07 & 325 & 600 & 20 & 30 & 460 \\
\hline 0.12 & 330 & 570 & 20 & 25 & 345 \\
\hline 0.24 & 330 & 570 & 20 & 25 & 153 \\
\hline 1 & 290 & 605 & $10^{-4}$ & 20 & 149 \\
\hline
\end{tabular}

TABLE S1. Parameters of the film growth on $\mathrm{Al}_{2} \mathrm{O}_{3}$ (0001): Temperatures of substrate and evaporator, pressure and the duration of the deposition. Thicknesses have been determined by a Veeco Dektak 150 Surface Profiler. 


\begin{tabular}{|l|l|l|l|l|l|}
\hline $\mathrm{Sb}_{2} \mathrm{Te}_{3}[\mathrm{mg}]$ & $\mathrm{Bi}_{2} \mathrm{Te}_{3}[\mathrm{mg}]$ & $\mathrm{Sb} 1-\mathrm{x}$ & $\mathrm{Bi} \mathrm{x}$ & $\mathrm{Te}$ [at-\%] & Film composition \\
\hline 200 & 0 & 1 & 0 & $59.7 \pm 1.8$ & $\mathrm{Sb}_{2} \mathrm{Te}_{3}$ \\
\hline 140 & 10 & 0.93 & $0.07 \pm 0.01$ & $60 \pm 1.5$ & $\left(\mathrm{Sb}_{0.93} \mathrm{Bi}_{0.07}\right)_{2} \mathrm{Te}_{3}$ \\
\hline 130 & 20 & 0.88 & $0.12 \pm 0.01$ & $60 \pm 1.5$ & $\left(\mathrm{Sb}_{0.88} \mathrm{Bi}_{0.12}\right)_{2} \mathrm{Te}_{3}$ \\
\hline 120 & 30 & 0.76 & $0.24 \pm 0.01$ & $60 \pm 1.5$ & $\left(\mathrm{Sb}_{0.76} \mathrm{Bi}_{0.24}\right)_{2} \mathrm{Te}_{3}$ \\
\hline 0 & 100 & 0 & 1 & $59.6 \pm 1.1$ & $\mathrm{Bi}_{2} \mathrm{Te}_{3}$ \\
\hline
\end{tabular}

TABLE S2. Amount of $\mathrm{Sb}_{2} \mathrm{Te}_{3}$ and $\mathrm{Bi}_{2} \mathrm{Te}_{3}$ powders [mg] used for the film growth on $\mathrm{Al}_{2} \mathrm{O}_{3}$ (0001) substrates and chemical composition [Sb and Bi content, Te in at-\%] of the resulting $\left(\mathrm{Sb}_{1-\mathrm{x}} \mathrm{Bi}_{\mathrm{x}}\right)_{2} \mathrm{Te}_{3}$ thin films. Composition data of solid solution samples is based on EDX of the $\mathrm{Bi} \mathrm{M}$ edge under the assumption that the Te to $(\mathrm{Sb}+\mathrm{Bi})$ ratio is unchanged, i.e. Te has 60 at$\%$. This assumption is required since due to peak overlap of Te with $\mathrm{Sb}$, both individual elements cannot be determined precisely.

\section{STEM simulation parameters:}

\begin{tabular}{|l|r|}
\hline Acceleration voltage & $300 \mathrm{kV}$ \\
\hline Defocus $\mathrm{C}_{1}$ & $0 \mathrm{~nm}$ \\
\hline Spherical aberration constant $\mathrm{C}_{3}$ & $1 \mathrm{~mm}$ \\
\hline Specimen thickness & $15 \mathrm{~nm}$ \\
\hline Emitter energy spread & $1 \mathrm{eV}$ \\
\hline Convergence angle & $10 \mathrm{mrad}$ \\
\hline
\end{tabular}

TABLE S3. Chosen parameters for the shown STEM simulations 


\section{Frequency dependent density of states used in our simulations:}

Fig. S1 shows experimental neutron incoherent scattering (NIS) data taken from literature ${ }^{[1]}$. Colored symbols indicate the frequency range, relevant for the c-axis phonon dispersion. The red solid lines show different polynomial fits that have been used for the density of states in our simulations. Small black dots mark angular frequency ranges that do not involve phonons along the c-axis.
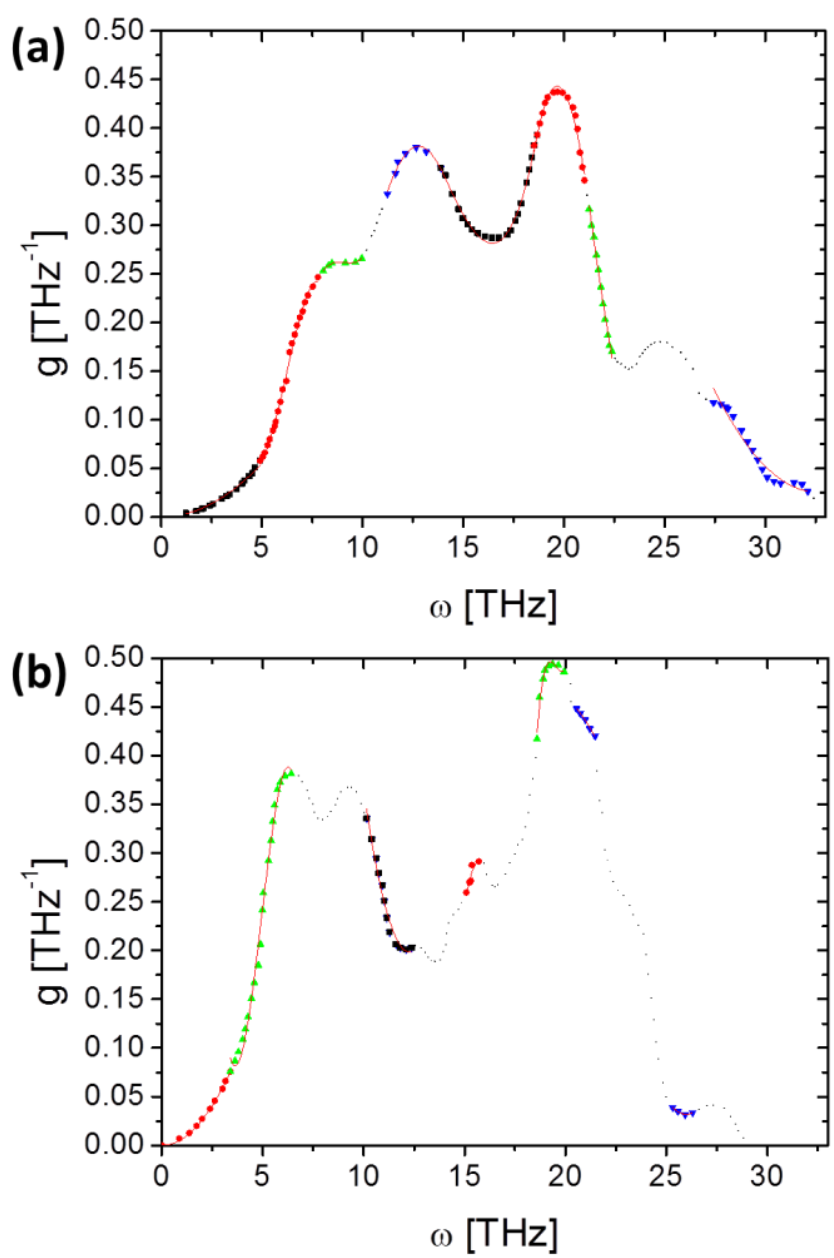

FIG. S1. Polynomial fits to NIS data ${ }^{[1]}$ in the angular frequency ranges that are relevant for the phonon dispersion along the c-axis. (a) For $\mathrm{Sb}_{2} \mathrm{Te}_{3}$ (b) For $\mathrm{Bi}_{2} \mathrm{Te}_{3}$ 
Frequency dependent and mode specific phonon velocities $v_{n}(\omega)$ derived from phonon dispersion relations:
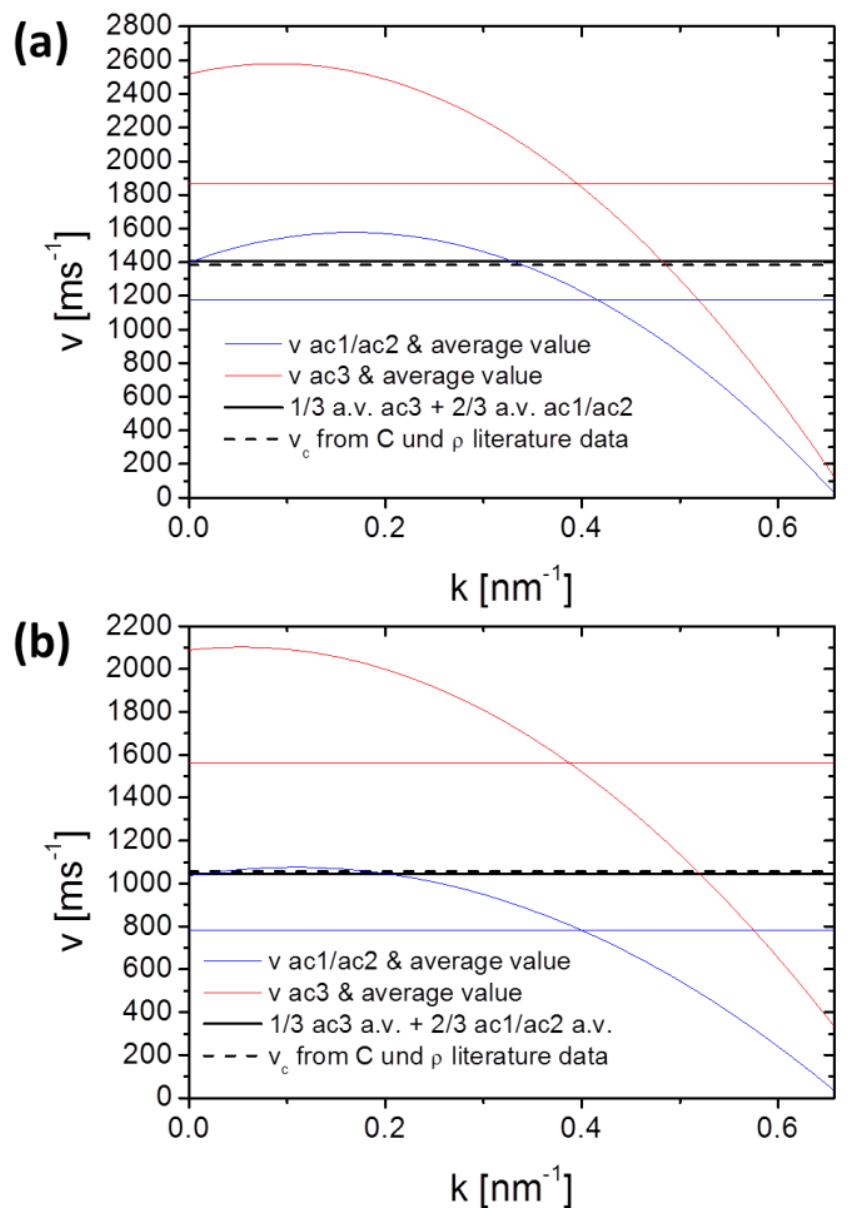

FIG. S2. Acoustic phonon velocities in the reciprocal space for (a) $\mathrm{Sb}_{2} \mathrm{Te}_{3}$ and (b) $\mathrm{Bi}_{2} \mathrm{Te}_{3}$. In addition, the average value of the transverse modes (ac1/ac2), of the longitudinal mode (ac3) and an average value of all modes (black line) are plotted, too. The black dashed line indicates the value of the sound velocity obtained from stiffness matrices and densities.

Dispersion relations obtained by first-principle calculations for $\mathrm{Sb}_{2} \mathrm{Te}_{3}{ }^{[2]}$ and for $\mathrm{Bi}_{2} \mathrm{Te}_{3}{ }^{[3]}$ have been used for the determination of $v_{n}(\omega)$. The total average values of $v_{n}(\omega)$ for the longitudinal and the two transverse acoustic modes are $1407 \mathrm{~ms}^{-1}$ for $\mathrm{Sb}_{2} \mathrm{Te}_{3}$ and $1043 \mathrm{~ms}^{-1}$ for $\mathrm{Bi}_{2} \mathrm{Te}_{3}$. These values are in good agreement with $v_{c}$ determined from the stiffness matrices $C^{[4]}$ and the mass densities ${ }^{[5]}, 1383 \mathrm{~ms}^{-1}$ for $\mathrm{Sb}_{2} \mathrm{Te}_{3}$ and $1057 \mathrm{~ms}^{-1}$ for $\mathrm{Bi}_{2} \mathrm{Te}_{3}$ (see Fig. S2). 


$$
\begin{gathered}
v_{a v, c}=\frac{1}{3} v_{a v, L}+\frac{2}{3} v_{a v, T} \\
v_{c}=\frac{1}{3} v_{L}+\frac{2}{3} v_{T}=\frac{1}{3} \sqrt{\left(\frac{C_{33}}{\rho}\right)}+\frac{2}{3} \sqrt{\left(\frac{C_{44}}{\rho}\right)}
\end{gathered}
$$

The usage of $v_{n}(\omega)$ instead of an average value is especially important for lowfrequency acoustic phonons, which are less affected by the Rayleigh scattering and thus dominate the thermal transport. The acoustic maximum velocities are around $2600 \mathrm{~ms}^{-1}$ for $\mathrm{Sb}_{2} \mathrm{Te}_{3}$ and around $2100 \mathrm{~ms}^{-1}$ for $\mathrm{Bi}_{2} \mathrm{Te}_{3}$. 


\section{X-ray diffraction results of the epitaxial thin films:}

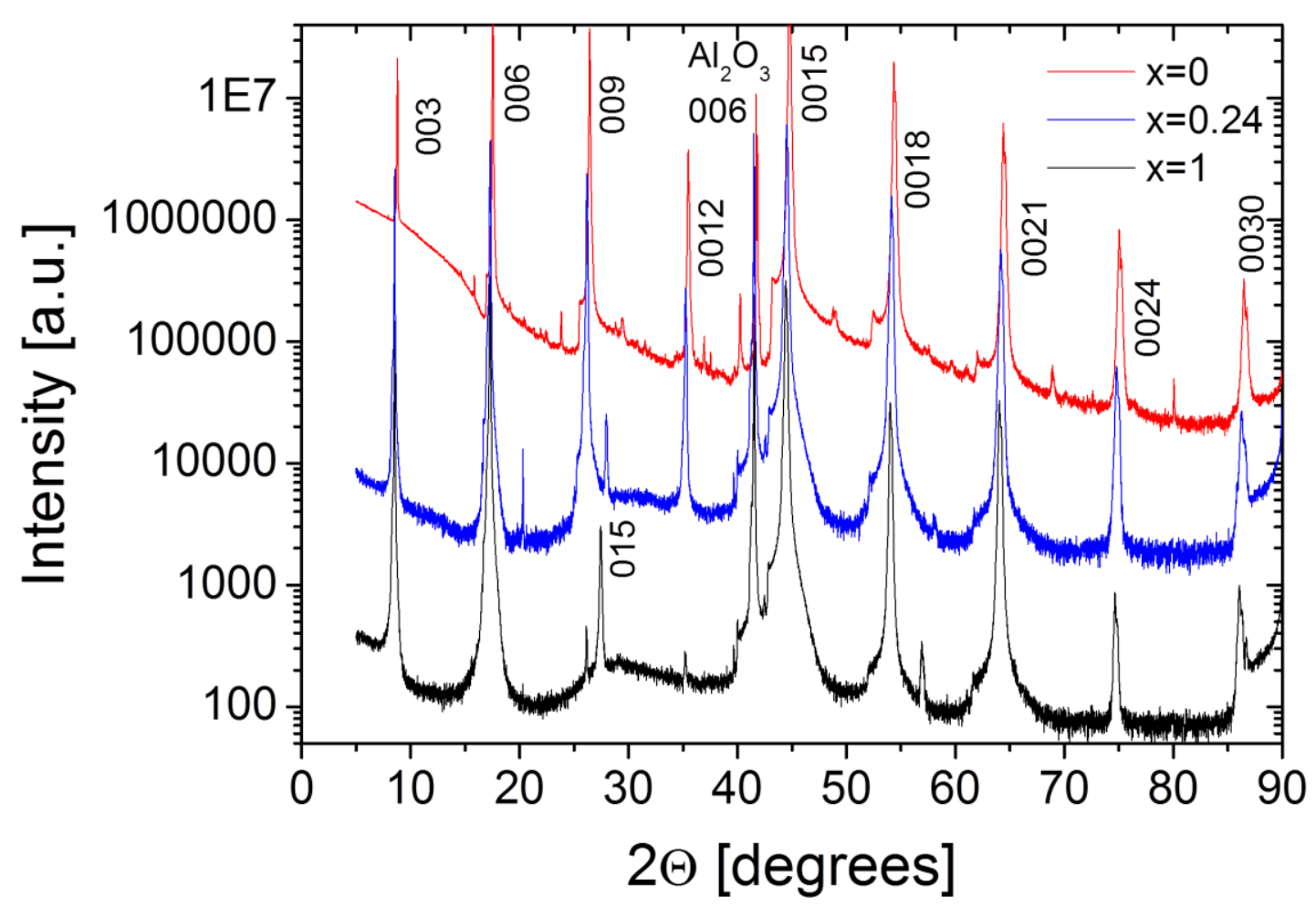

FIG. S3. X-ray diffractogram of PVD-grown epitaxial $\mathrm{Bi}_{2} \mathrm{Te}_{3}, \mathrm{Sb}_{2} \mathrm{Te}_{3}$ and solid solution thin films deposited on c-orientated sapphire. For better visibility, the values of the $x=0$ and the $x=0.24$ sample have been multiplied with constant factors. With increasing Bi content, the positions of the $00 l(l=3,6,9 \ldots)$ reflections shift to slightly lower $2 \Theta$ values due to the expansion of the crystal lattice proving the successful substitution of $\mathrm{Sb}$ by larger Bi atoms. The XRD pattern of $\left(\mathrm{Sb}_{0.76} \mathrm{Bi}_{0.24}\right)_{2} \mathrm{Te}_{3}$ and $\mathrm{Bi}_{2} \mathrm{Te}_{3}$ films show the presence of the 015 reflection with very low intensity around $2 \Theta=27^{\circ}$ caused by the presence of very few freestanding crystallites of the film surface. These crystallites are visible in the SEM pictures in Fig. S4 (b). Please note that the intensity of the 009 and the 0012 reflections of $\mathrm{Bi}_{2} \mathrm{Te}_{3}$ are very low compared to the corresponding reflections of $\mathrm{Sb}_{2} \mathrm{Te}_{3}$ and $\left(\mathrm{Sb}_{0.76} \mathrm{Bi}_{0.24}\right)_{2} \mathrm{Te}_{3}$. This is in good agreement with the reported XRD pattern in PDF 15-863 $\left(\mathrm{Bi}_{2} \mathrm{Te}_{3}\right)$ and PDF 15-874 $\left(\mathrm{Sb}_{2} \mathrm{Te}_{3}\right)$. 


\section{Scanning Electron Microscopy of the thin films:}

Fig. S4 shows the surfaces of the $\mathrm{Sb}_{2} \mathrm{Te}_{3}$ and the $\mathrm{Bi}_{2} \mathrm{Te}_{3}$ films, where also the TEM and $3 \omega$ data (see main text) has been obtained. The surface of the thicker $\mathrm{Sb}_{2} \mathrm{Te}_{3}$ film is very smooth while that of the $\mathrm{Bi}_{2} \mathrm{Te}_{3}$ film additionally shows pinholes and a few misoriented crystallites.

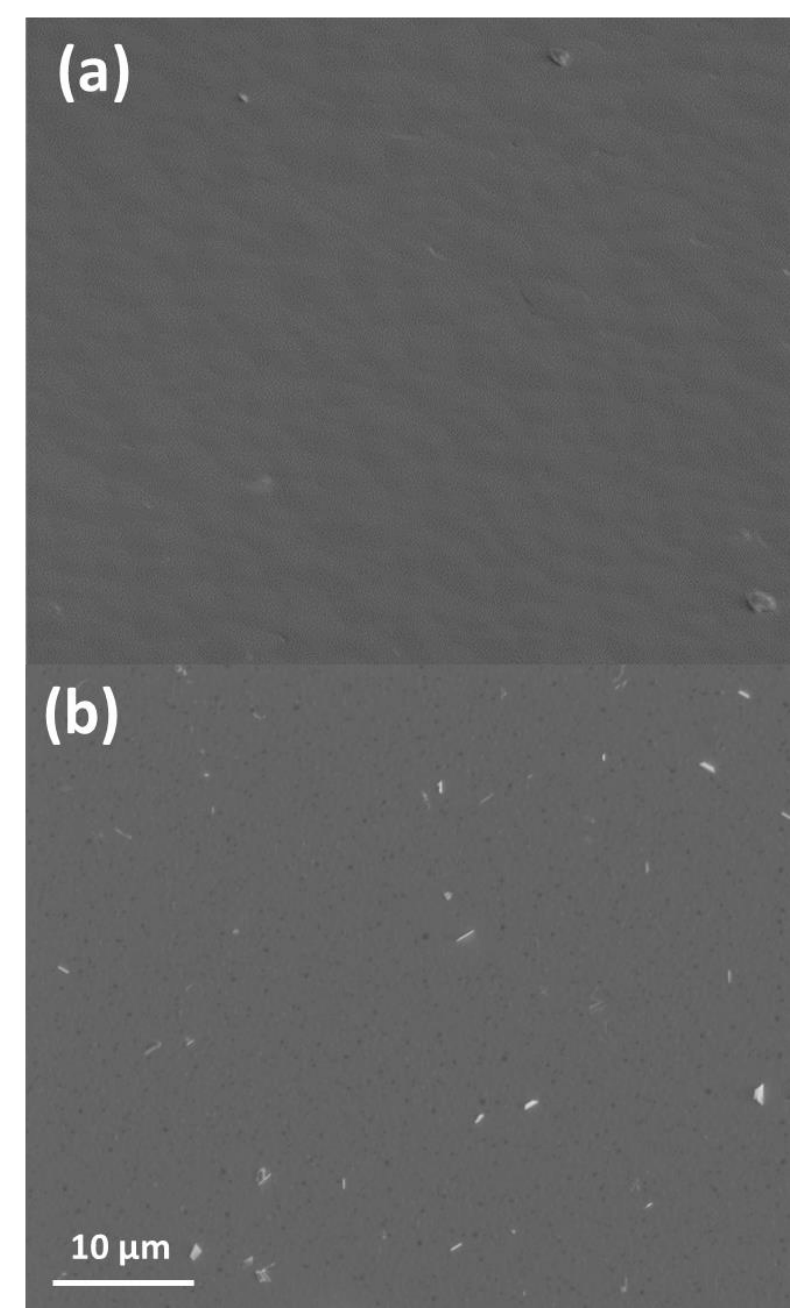

FIG. S4. SEM pictures of the surfaces (a) of a $1184 \mathrm{~nm}$ thick $\mathrm{Sb}_{2} \mathrm{Te}_{3}$ thin film and (b) of a 149 $\mathrm{nm}$ thick $\mathrm{Bi}_{2} \mathrm{Te}_{3}$ thin film deposited on c-orientated sapphire. 


\section{Additional High-Resolution Transmission Electron Microscopy:}
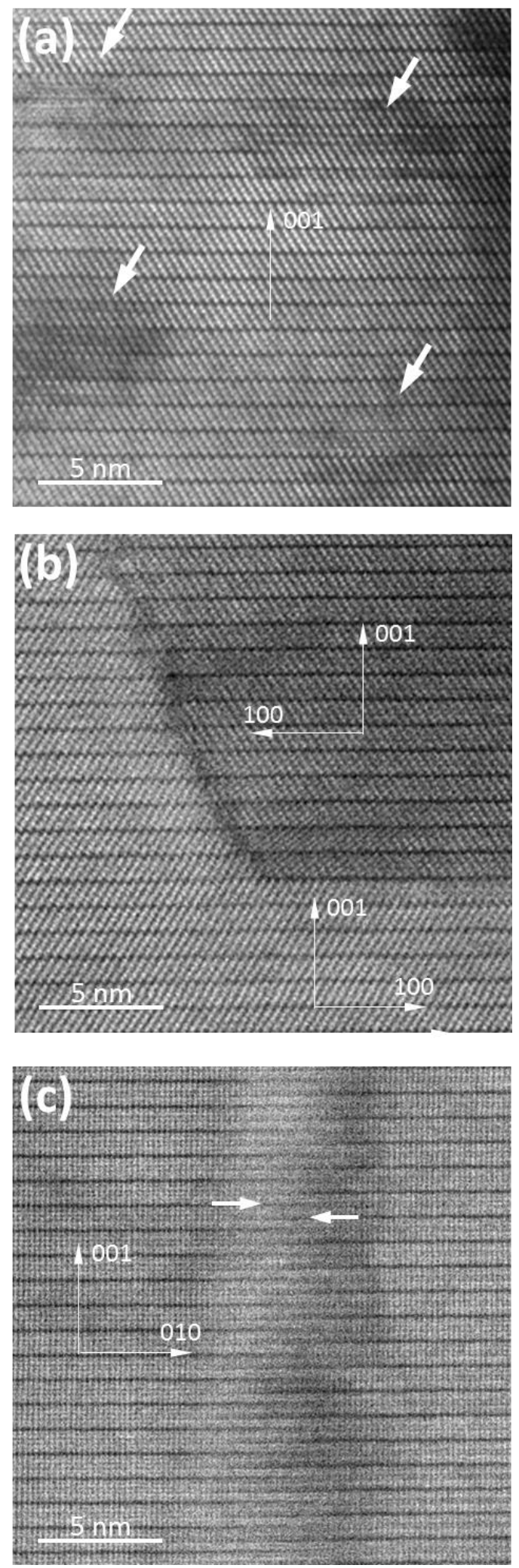

FIG. S5. HRSTEM images of various defects found in the $\mathrm{Sb}_{1-\mathrm{x}} \mathrm{Bi}_{\mathrm{x}} \mathrm{Te}_{3}$ films: (a) stacking faults marked with the white arrows in the undoped $\mathrm{Sb}_{2} \mathrm{Te}_{3}$ film; (b) a large twin boundary in $\mathrm{Sb}_{0.88} \mathrm{Bi}_{0.12} \mathrm{Te}_{3}$ film; (c) antiphase boundary in $\mathrm{Sb}_{0.76} \mathrm{Bi}_{0.24} \mathrm{Te}_{3}$ film. The shift of two domains along the [001] direction is indicated with the white arrows. 

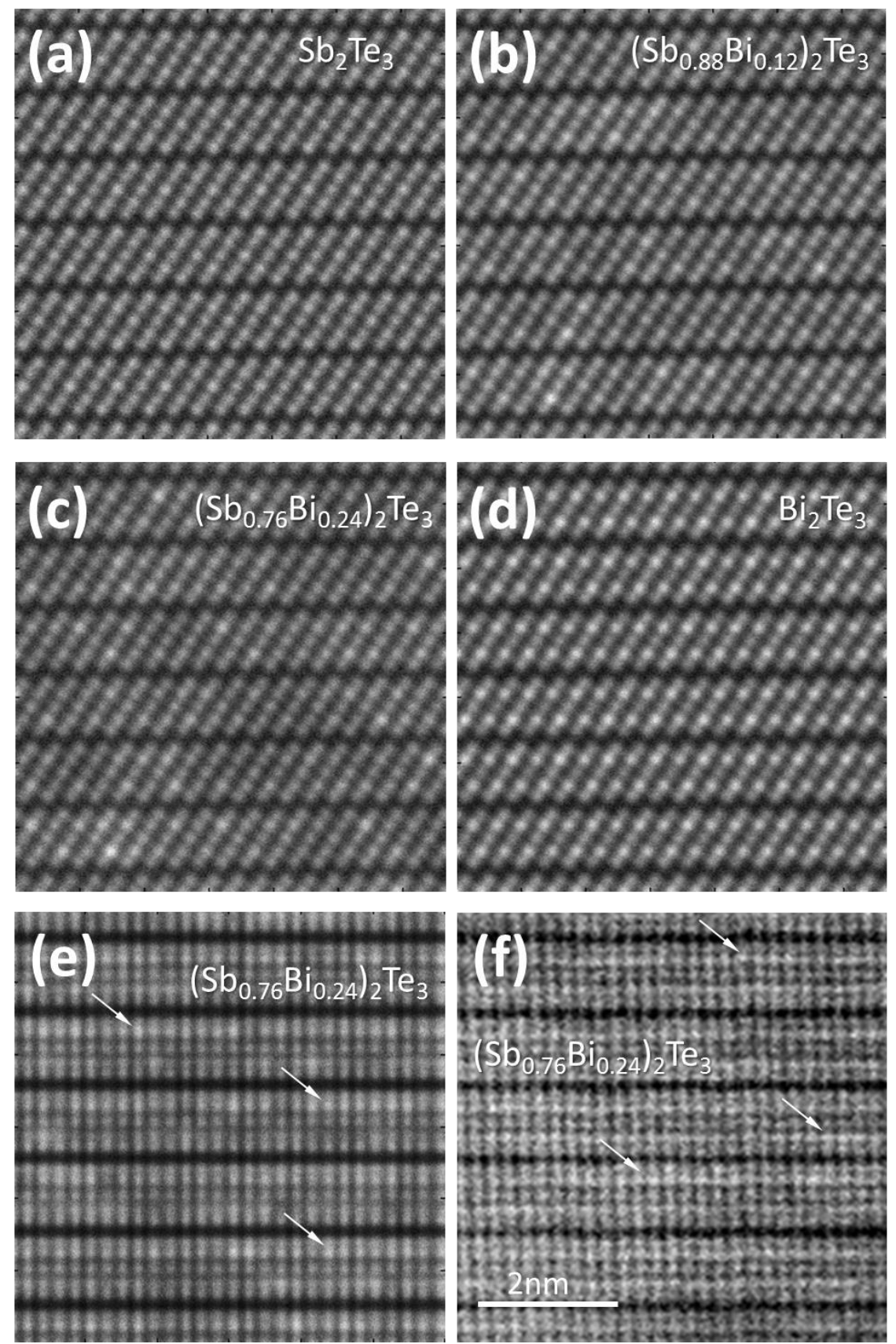

FIG. S6. STEM-HAADF images of samples with different Bi concentrations

(a)-(d) Calculated STEM-HAADF images (zone axis [010])

(e) Calculated STEM-HAADF image for $x=0.24$ (zone axis [100])

(f) Experimental STEM-HAADF image for $x=0.24$ (zone axis [100]) 
CHAPTER 5 : Publication 2 (Supplementary material)
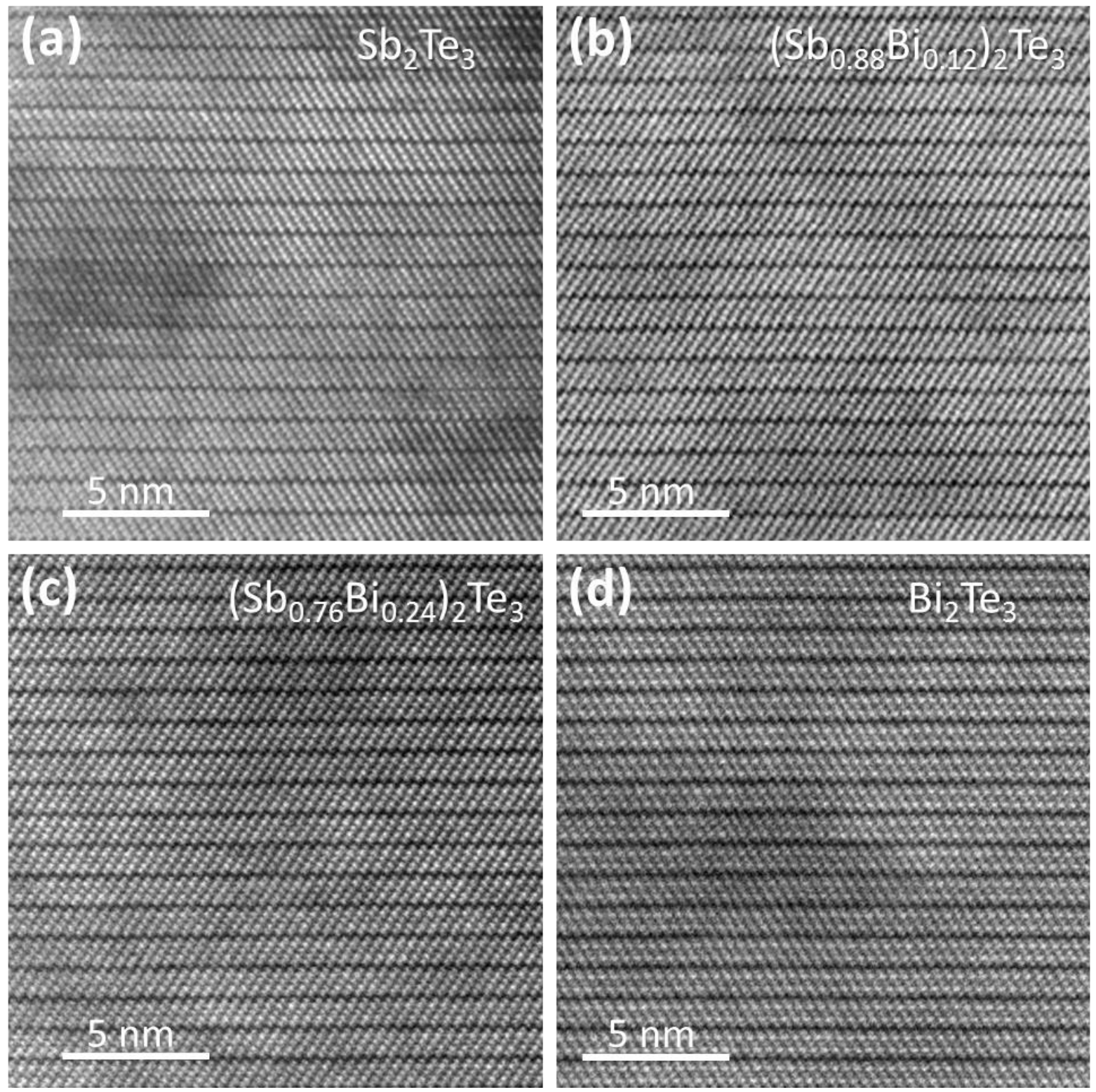

FIG. S7. STEM-HAADF images of typical areas in samples with different Bi concentrations. The STEM-HAADF combined to x-ray diffraction confirms high crystallinity and low defect concentrations. 

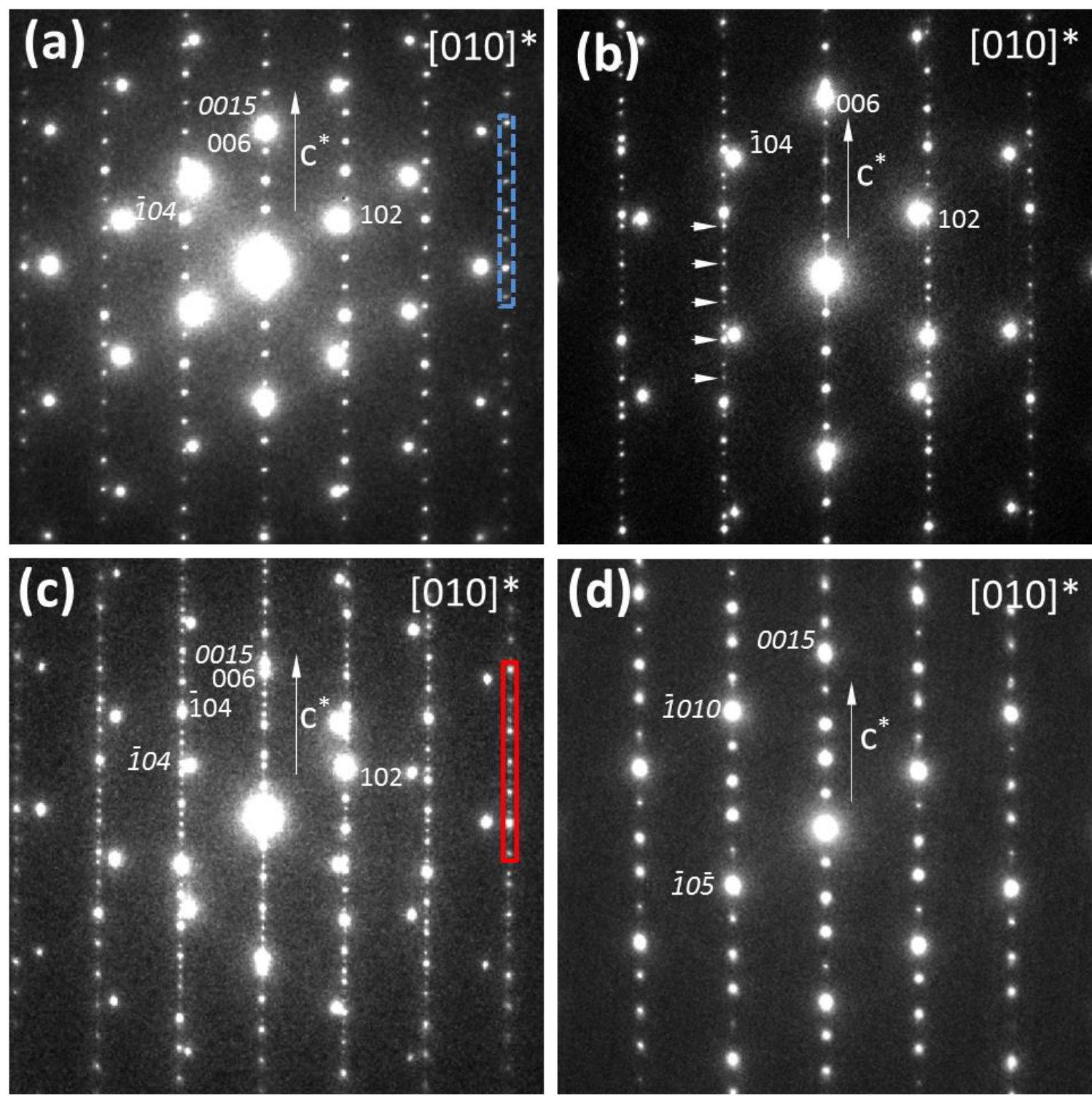

FIG. S8. Selected area diffraction patterns from pure $\mathrm{Sb}_{2} \mathrm{Te}_{3}(\mathbf{a}), \mathrm{Sb}_{2} \mathrm{Te}_{3}$ doped with $12 \%$ of $\mathrm{Bi}(\mathbf{b}), \mathrm{Sb}_{2} \mathrm{Te}_{3}$ doped with $24 \%$ of $\mathrm{Bi}$ (c), and pure $\mathrm{Bi}_{2} \mathrm{Te}_{3}$ (d). All SAEDs demonstrate high quality epitaxial growth of films on [001] $\mathrm{Al}_{2} \mathrm{O}_{3}$ substrate. The spots coming from the film are denoted with italic. The presence of large twinned domains in the films is revealed by the presence of additional spots, e.g. in (b), marked with the arrowheads. The only SAED pattern from the $\mathrm{Bi}_{2} \mathrm{Te}_{3}$ is given in (d). The blue / red boxes in (a) / (c) indicate the regions used for the intensity profile analysis in Fig. 3. 


\section{Detailed description of the performed subtraction of the electronic contribution to the thermal conductivity from our $3 \omega$ results:}

For $x=0\left(\mathrm{Sb}_{2} \mathrm{Te}_{3}\right) \kappa_{a b, e}(T)$ and $\kappa_{c, e}(T)$ in the range of $T=100$ to $300 \mathrm{~K}$ have been obtained from experimental resistivity data ${ }^{[6]}$ using the Wiedemann-Franz law and

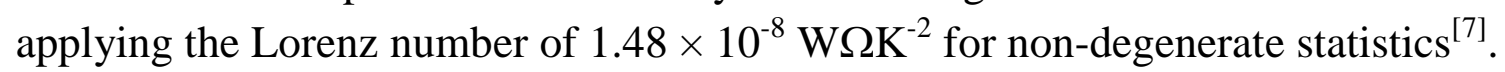

For the doped systems, no temperature dependent $\kappa_{c, e}(T)$ has been found in literature. For $x=0.07$ and $x=0.12$, we use the progression of $\kappa_{c, e}(T)$ at $\mathrm{x}=0$ normalized to the $\mathrm{x}$ dependent $\kappa_{a b, e}(300 K)$ which are calculated from the $\sigma_{a b}(300 K)$ values published by Jeon et al. ${ }^{[8]}$.

For $\mathrm{x}=0.24$, the progression of $\kappa_{c, e}(T)$ at $\mathrm{x}=0$ has been scaled down to the room temperature value of $\kappa_{c, e}=0.19 \mathrm{Wm}^{-1} \mathrm{~K}^{-1}$ at $x=0.25$ given by ref. ${ }^{[9]}$.

For $x=1\left(\mathrm{Bi}_{2} \mathrm{Te}_{3}\right)$, we determined $\kappa_{c, e}(T)$ by combining $\kappa_{a b, e}(T)$ data calculated out of $\sigma_{a b}(T)^{[8]}$ with anisotropy data $\eta_{\sigma}(T)$ published by Nagao et al. ${ }^{[10]}$.

The resulting $\kappa_{c, e}(T), \kappa_{a b, e}(T)$ and anisotropy $\eta_{\sigma}(T)$ for $\mathrm{Sb}_{2} \mathrm{Te}_{3}$ and $\mathrm{Bi}_{2} \mathrm{Te}_{3}$ is shown in Figs. S9, S10 and S11.

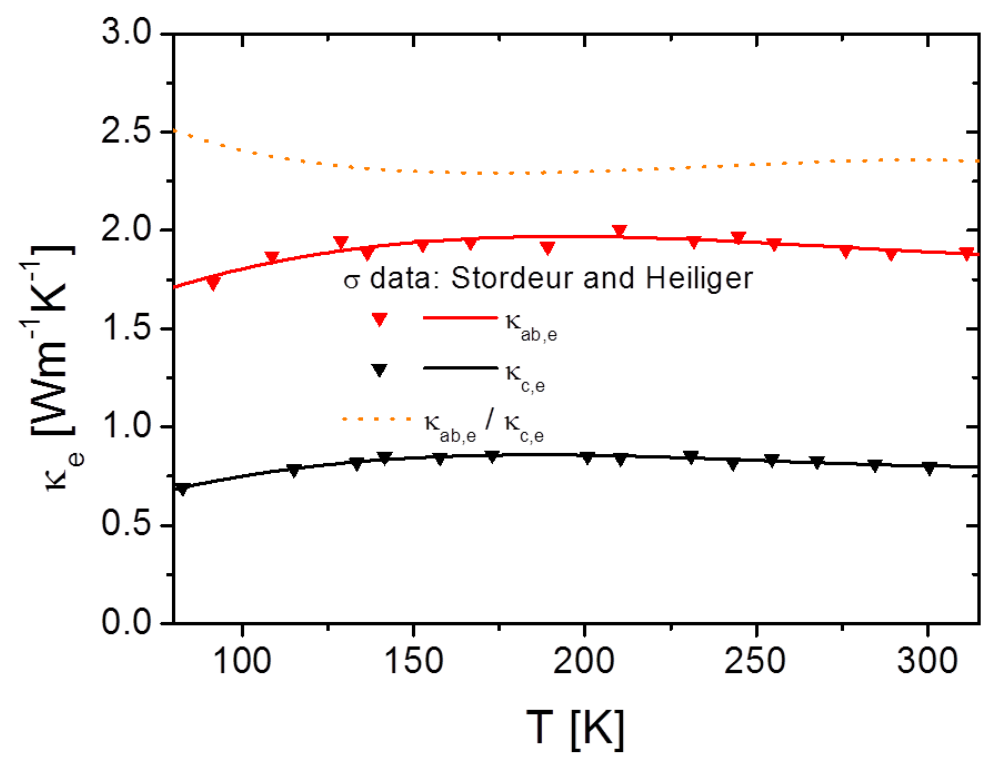

FIG. S9. $\mathrm{Sb}_{2} \mathrm{Te}_{3}: \kappa_{e}(T)$ calculated out of $\sigma_{a b}(T)$ and $\sigma_{c}(T)$ taken from Stordeur and Heiliger $^{[6]}$ and polynomial fits used in this work. The shown anisotropy $\eta_{\sigma}(T)$ is obtained by dividing the the two fitted $\kappa_{a b, e}(T)$ and $\kappa_{c, e}(T)$ curves. 

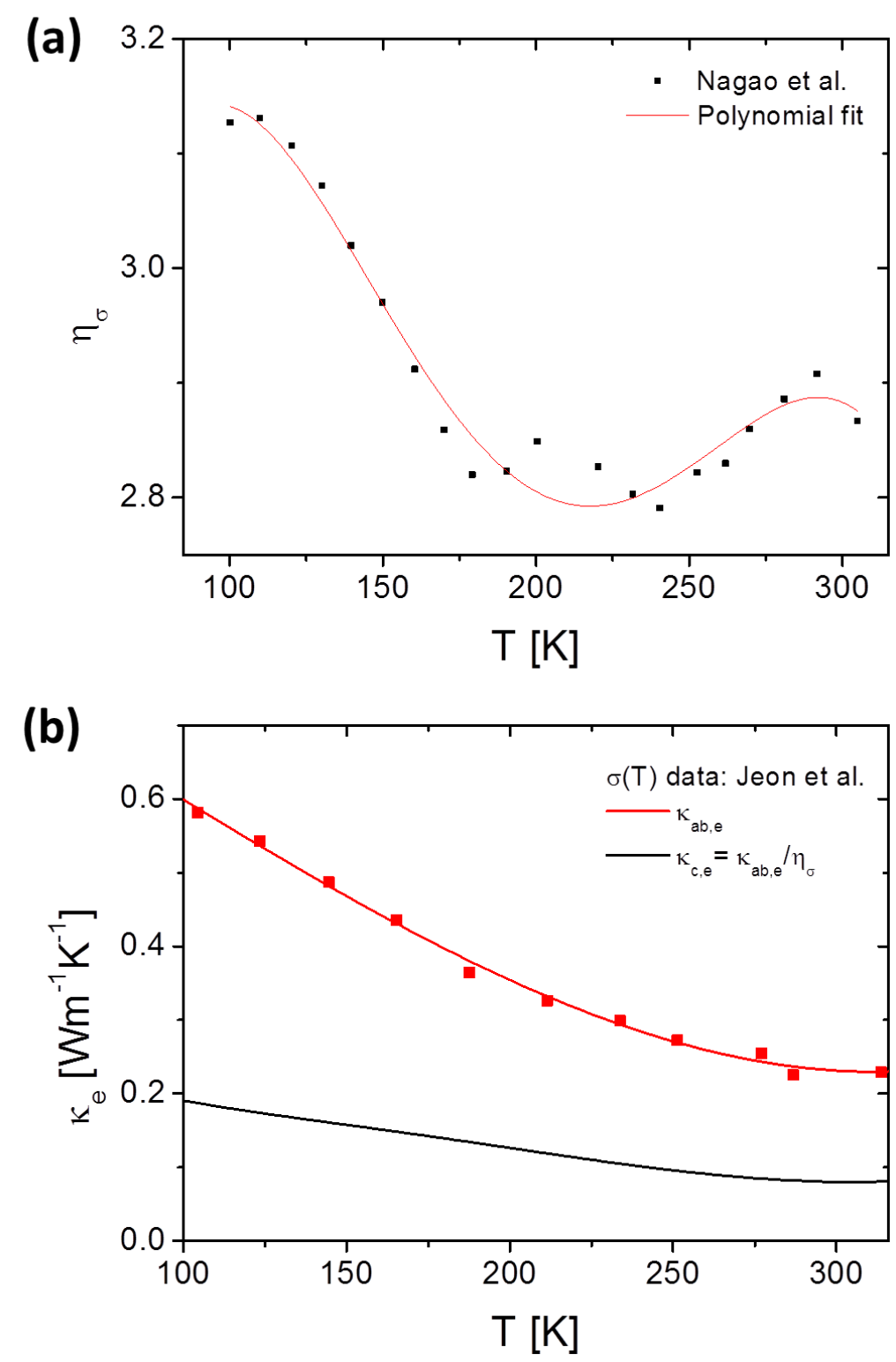

FIG. S10. $\mathrm{Bi}_{2} \mathrm{Te}_{3}$ : (a) Anisotropy of the electrical conductivity $\eta_{\sigma}(T)$ taken from Nagao et al. ${ }^{[10]}$ including the polynomial fit used in this work. (b) $\kappa_{a b, e}(T)$ data calculated out of $\sigma_{a b}(T)$ taken from ref. ${ }^{[8]}$ including polynomial fit and $\kappa_{c, e}(T)=\kappa_{a b, e}(T) / \eta_{\sigma}(T)$ using the fitting curves. 


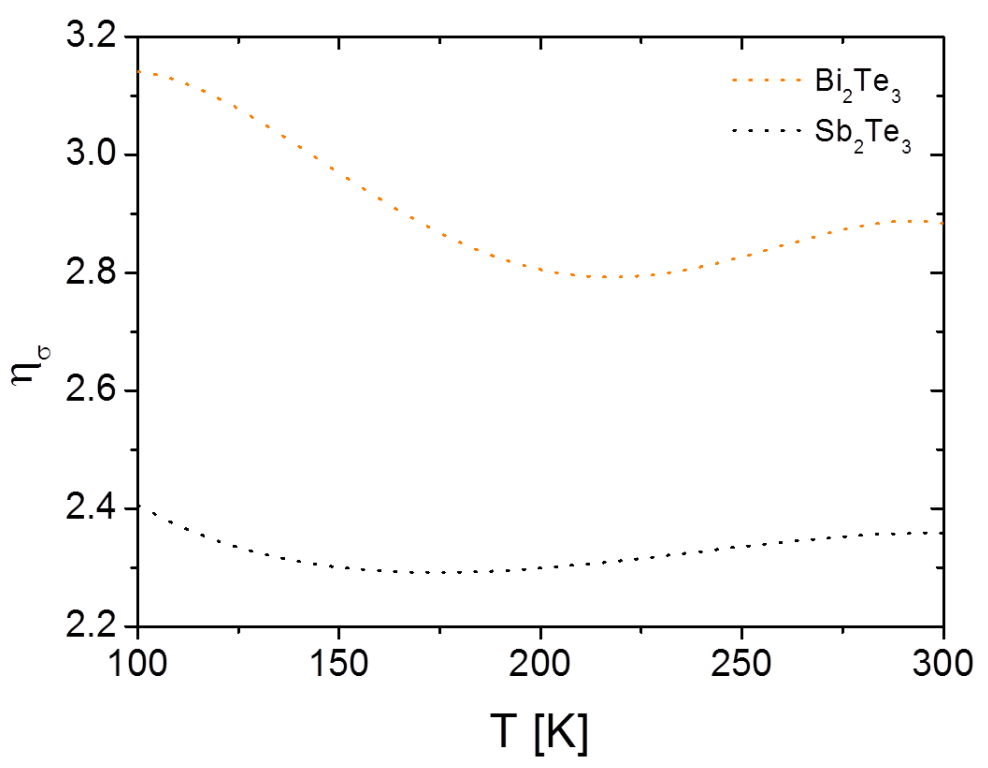

FIG. S11. Anisotropies of the electrical conductivity $\eta_{\sigma}(T)$ of $\mathrm{Sb}_{2} \mathrm{Te}_{3}$ and $\mathrm{Bi}_{2} \mathrm{Te}_{3}$ (see Figs. S9 and S10) for direct comparison.

\section{Frequency dependence of lattice thermal conductivity for all samples:}

For every measured epitaxial film, Figs. S12 - S16 show (a) the differential lattice thermal conductivity of phonons in a specific frequency range

$$
\kappa_{c, l, \omega}(T)=g(\omega) C(\omega, T) v_{n}(\omega) l_{m f p}(\omega, T)
$$

with

$$
\kappa_{c, l}(T)=\sum_{n} \int_{0}^{\omega} d \omega \kappa_{c, l, \omega}(T)
$$

and (b) the integrated values shown in (a) at 3 different temperatures. The values are based on our simulation results. It allows a detailed insight of the contribution of the different phonon modes and frequency ranges as a function of temperature and doping level. One may notice that big temperature dependent differences only occur at low frequencies, where the Umklapp scattering is dominant, whereas for high temperatures the Rayleigh scattering is dominant. At higher frequencies, lattice thermal conductivity is increased due to an increase in the specific heat capacity. However, this is overcompensated by the very small mean free path and thus high frequency optical phonons play only a minor role. Furthermore, if the mean free path approaches the phonon wavelength, the phonon picture is only of limited validity. 

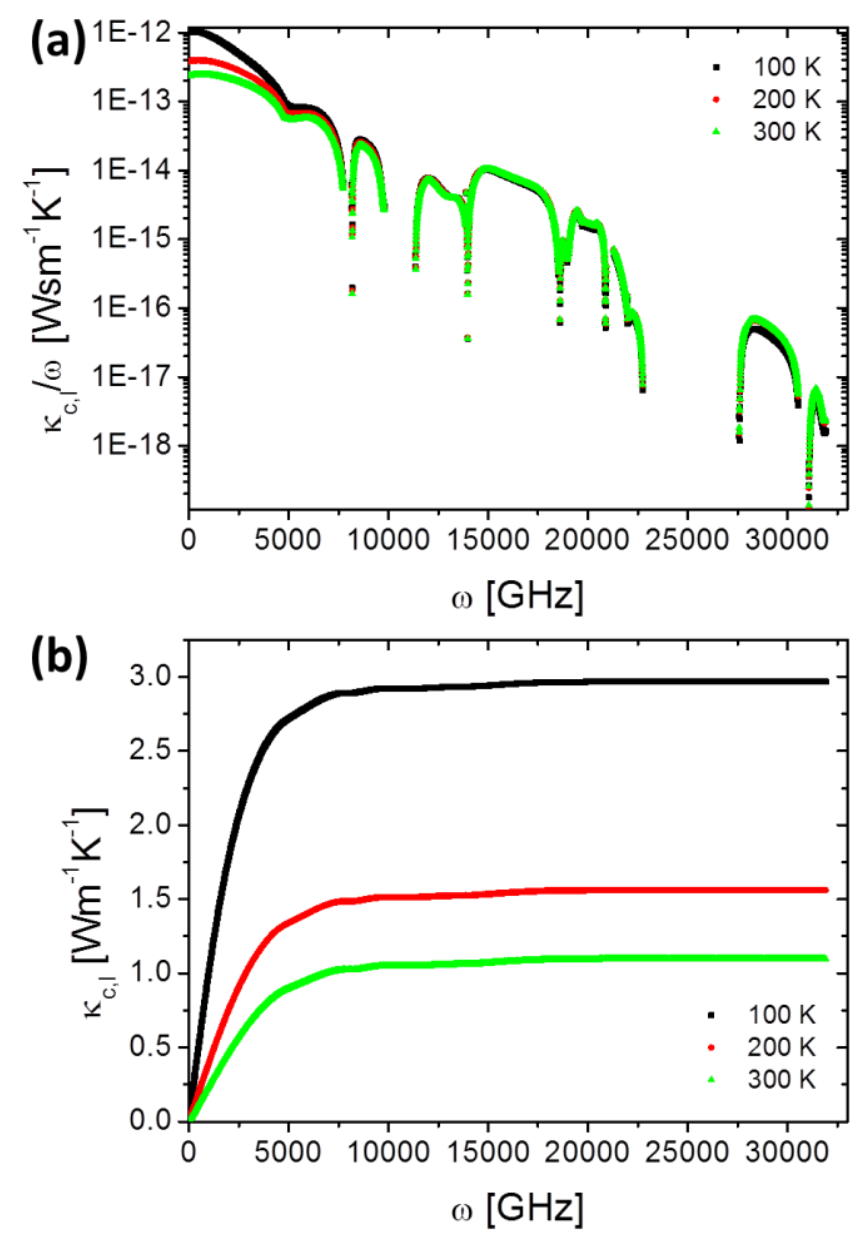

FIG. S12. $x=0$. (a) Differential lattice thermal conductivity per angular frequency vs. angular frequency. (b) Total lattice thermal conductivity reached at a specific angular frequency. 

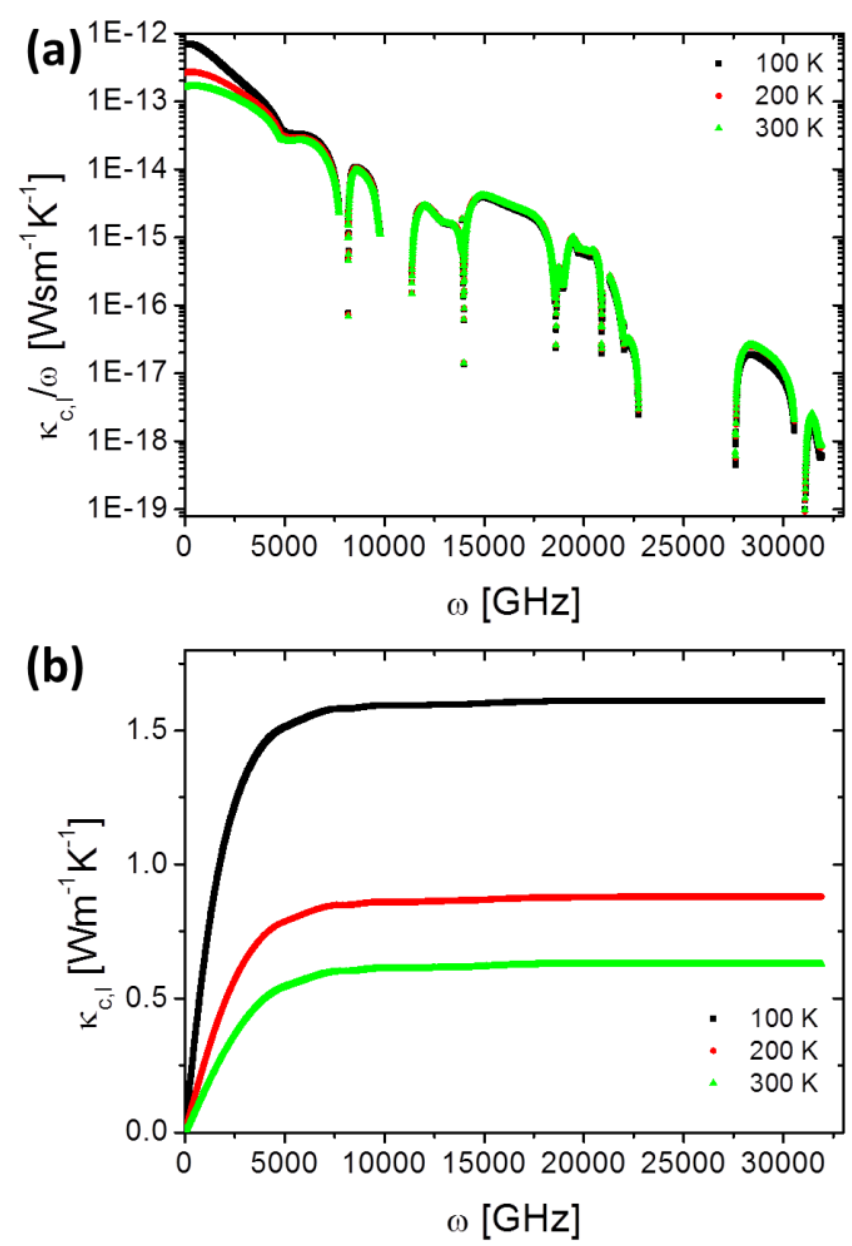

FIG. S13. $x=0.07$. (a) Differential lattice thermal conductivity per angular frequency vs. angular frequency. (b) Total lattice thermal conductivity reached at a specific angular frequency. 

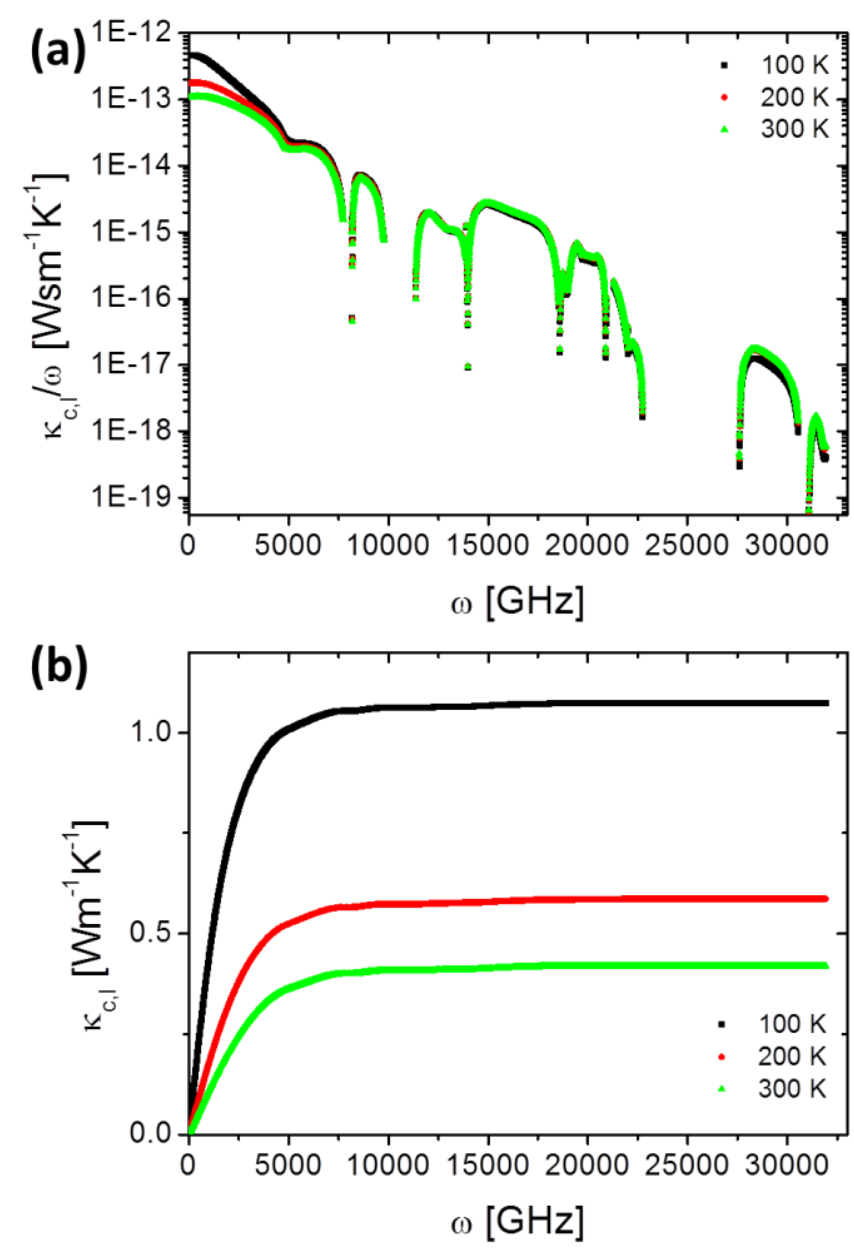

FIG. S14. $x=0.12$. (a) Differential lattice thermal conductivity per angular frequency vs. angular frequency. (b) Total lattice thermal conductivity reached at a specific angular frequency. 

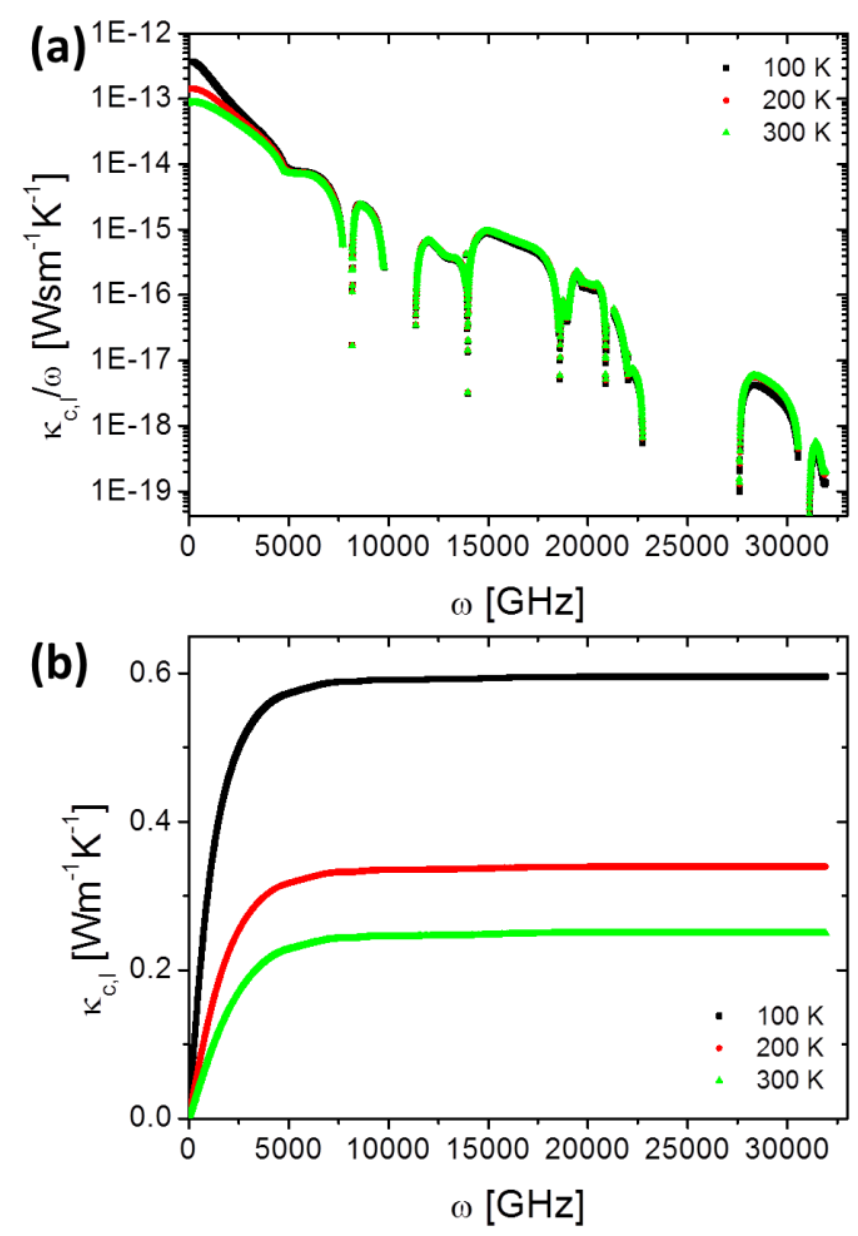

FIG. S15. $x=0.24$. (a) Differential lattice thermal conductivity per angular frequency vs. angular frequency. (b) Total lattice thermal conductivity reached at a specific angular frequency. 

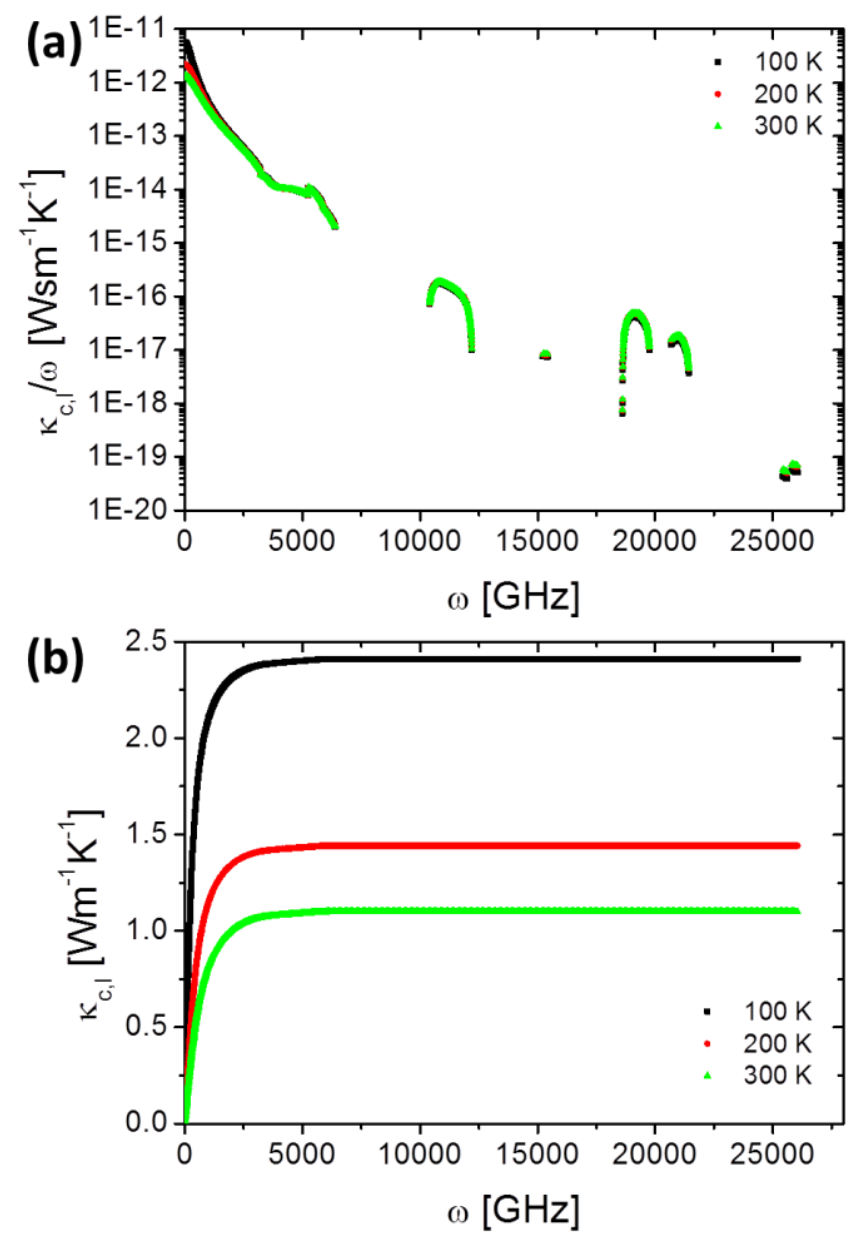

FIG. S16. $x=1$. (a) Differential lattice thermal conductivity per angular frequency vs. angular frequency. (b) Total lattice thermal conductivity reached at a specific angular frequency. 


\section{Frequency and doping dependent contributions of Umklapp and Rayleigh scattering:}

In Figs. S17 and S18 the total mean free path together with the contributions by Umklapp and Rayleigh scattering, respectively, is plotted versus the angular frequency. Clearly, at increased temperatures the frequency range where Umklapp scattering is the dominant scattering mechanism is larger. As we show in this work, at $x=0.24$ already at around $170 \mathrm{~K}$ the combination of strong Umklapp scattering at low and medium frequencies and the strong Rayleigh scattering at medium and high frequencies leads to a phonon glass like behavior of the lattice thermal conductivity over a broad temperature range up to room temperature and most likely beyond that.
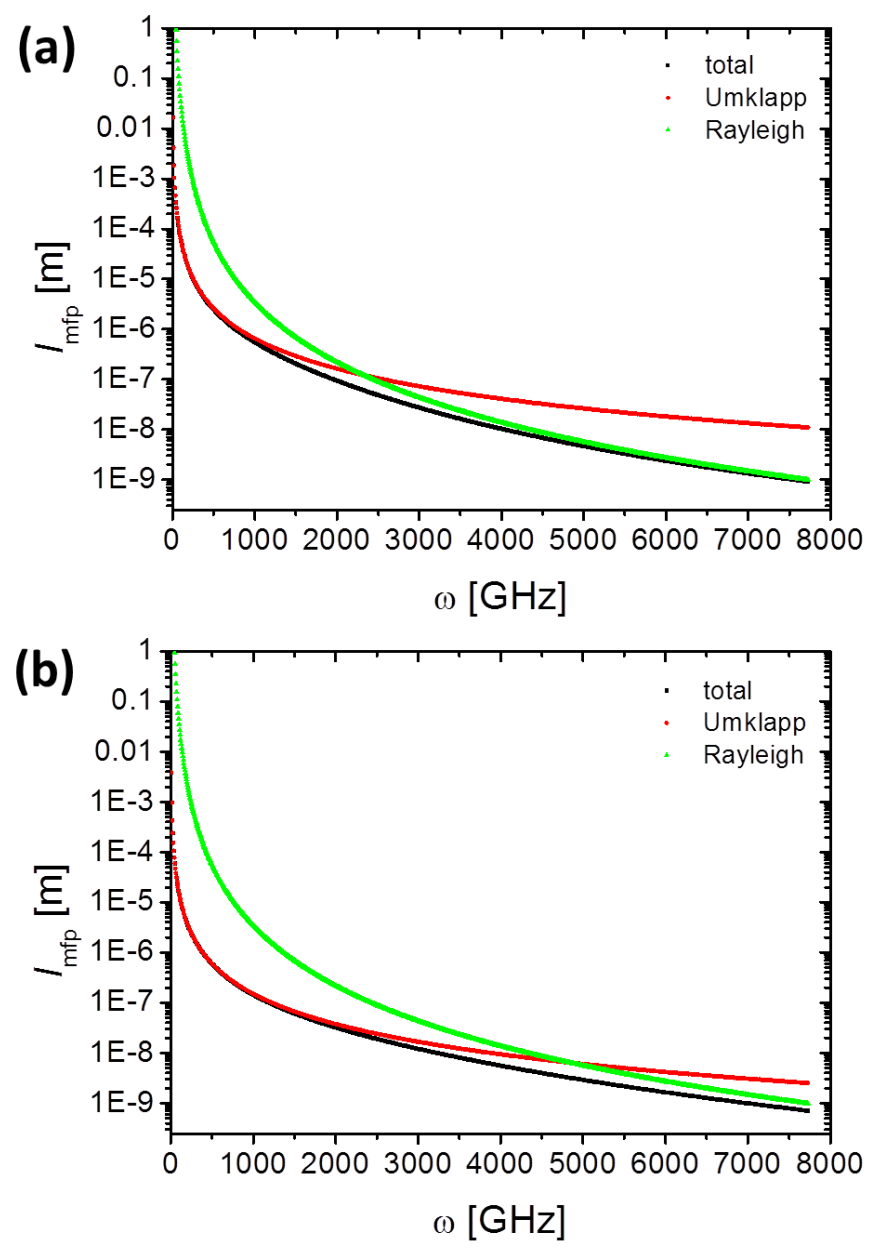

FIG. S17. $x=0$. Total, Umklapp and Rayleigh phonon mean free path vs. angular frequency at (a) $100 \mathrm{~K}$ (b) $300 \mathrm{~K}$. 

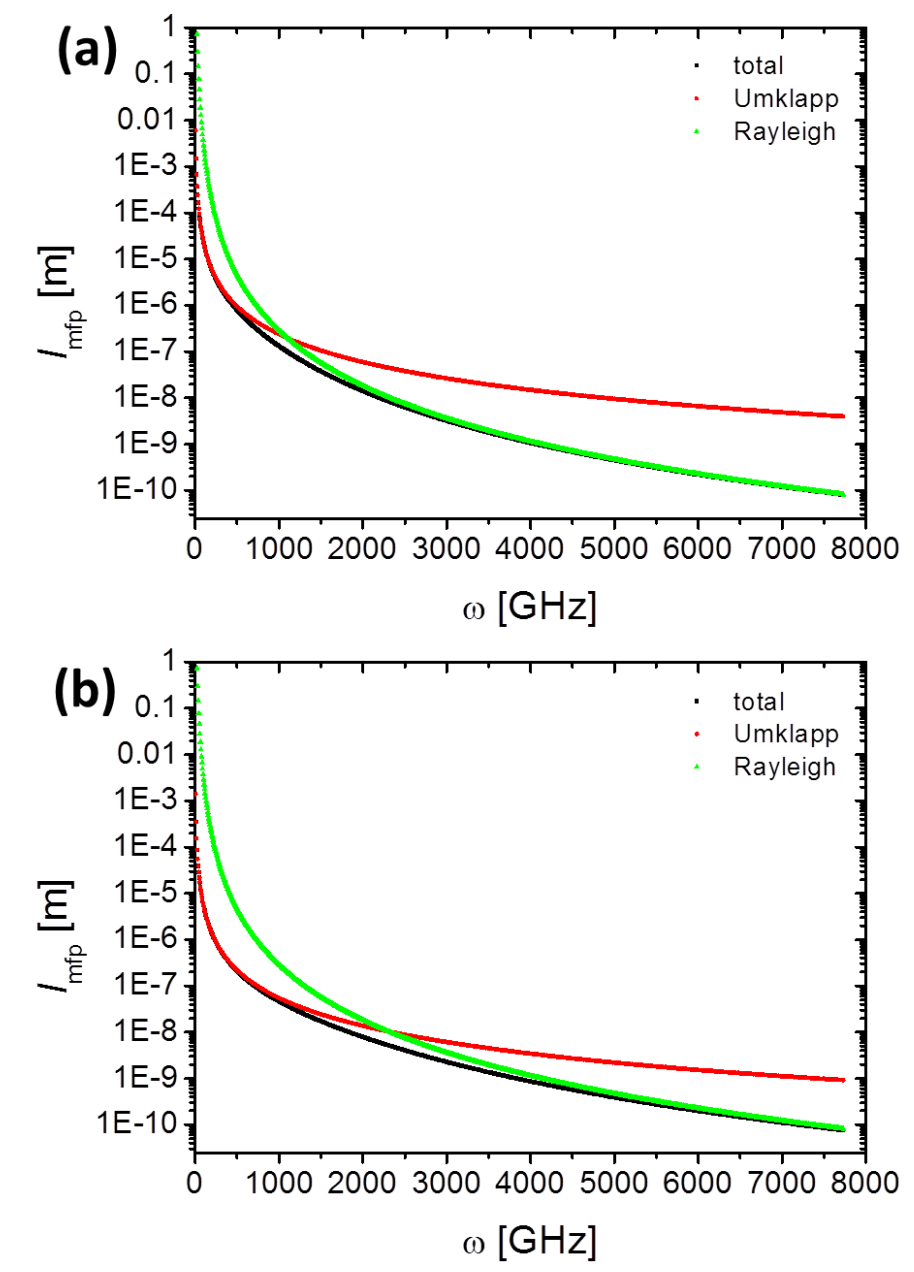

FIG. S18. $x=0.24$. Total, Umklapp and Rayleigh phonon mean free path vs. angular frequency at (a) $100 \mathrm{~K}$ (b) $300 \mathrm{~K}$.

[1] H. Rauh, R. Geick, H. Köhler, N. Nücker, and N. Lehner, J. Phys. C: Solid State Phys. 14, 2705 (1981).

[2] B.-T. Wang, P. Souvatzis, O. Eriksson, and P. Zhang, J. Chem. Phys. 142, 174702 (2015).

[3] O. Hellman and D. A. Broido, Phys. Rev. B 90, 134309 (2014).

[4] M. de Jong, W. Chen, T. Angsten, A. Jain, R. Notestine, A. Gamst, M. Sluiter, C. Krishna Ande, S. van der Zwaag, J. J. Plata, C. Toher, S. Curtarolo, G. Ceder, K. A. Persson, and M. Asta, Scientific Data 2, 150009 (2015).

[5] W. M. Haynes, CRC Handbook of Chemistry and Physics, CRC Press, Boca Raton, FL, 97th edition 2016.

[6] M. Stordeur and W. Heiliger, Phys. Stat. Sol. B 78, K103 (1976).

[7] R. Lan, R. Endo, M. Kuwahara, Y. Kabayashi, and M. Susa, J. Electron. Mater. 46(2), 955 (2017).

[8] H.-W. Jeon, H.-P. Ha, D.-B. Hyun, and J.-D. Shim, J. Phys. Chem. Solids 52(4), 579 (1991).

[9] A. Jacquot, N. Farag, M. Jaegle, M. Bobeth, J. Schmidt, D. Ebling, and H. Böttner, J. Electron. Mater. 39(9), 1861 (2010).

[10] J. Nagao, M. Ferhat, E. Hatta, and K. Mukasa, Phys. Stat. Sol. B 219, 347 (2000). 



\section{CHAPTER 6}

\section{General Discussion and Summary}

The present thesis in a systematical way combines detailed structural analysis and thermal transport measurements of epitaxially grown $\left(\mathrm{Sb}_{1-\mathrm{x}} \mathrm{Bi}_{\mathrm{x}}\right)_{2} \mathrm{Te}_{3}$ films. This chapter is dedicated to a discussion of the presented publications and their meaning for future thermoelectric research.

\section{Chapter 4: Publication 1}

Publication 1 deals with the detailed investigation of $\mathrm{Sb}_{2} \mathrm{Te}_{3}$ films grown on $\alpha-\mathrm{Al}_{2} \mathrm{O}_{3}$ substrates. To the best knowledge of the author, this constitutes the first time of obtaining epitaxial $\mathrm{Sb}_{2} \mathrm{Te}_{3}$ films by the co-evaporation method and the first time of $\kappa_{c}$ measurements of $\mathrm{Sb}_{2} \mathrm{Te}_{3}$ over the temperature range from 100 to $300 \mathrm{~K}$ in combination with HRTEM investigations.

Method development and results gave rise to and enabled further research on Bi-doped solid solution samples.

Method development: $3 \omega$ measurement and improved analysis. The $3 \omega$ method is an established method for precise thermal conductivity measurements. Especially for the temperature dependent characterization of thin films it is often the method of choice. However, it is an indirect method which requires an elaborate evaluation process. The common analysis works very well and has been proven at quite a few substrate-film systems, but the required boundary conditions for solving the underlying mathematical equations lead to limited application conditions.

The results shown in this thesis have been obtained by using a $3 \omega$ setup built by Thilo Kramer and Jonas Lindner. The conventional data evaluation has been modified and extended for highly anisotropic thin film materials. The new evaluation routine allows the precise measurement of films with relatively high thermal conductivities compared to the substrate which is especially important for high temperature measurements. The refined evaluation also increases the precision at low temperatures, where common single crystalline substrate materials usually show high thermal conductivities, by taking into account substrate backside boundary conditions. An important side effect of the used evaluation is the error-prone determination of phase shifts getting dispensable. 
The author has developed a sputtering procedure by means of which the deposition of a well defined insulating a- $\mathrm{SiO}_{2}$ layer on the unheated sample/substrat is possible, immediately followed by the deposition of $\mathrm{Pt}$ for the $3 \omega$ heating structure. In combination with a special sample and heating structure geometry this approach enables a high reproducibility and reliability of the insulation. It is highly likely that this method and know-how development will be of significant benefit for future investigations of different thin film materials.

$\mathrm{Sb}_{2} \mathrm{Te}_{3} / \alpha-\mathrm{Al}_{2} \mathrm{O}_{3}$ interface: Kapitza resistances. Accurate measurements of the intrinsic cross plane thermal conductivity require the precise measurement of the Kapitza resistances occurring at the interfaces between heater, insulation layer, layer of interest and substrate. The thermal boundary resistances have been determined by different thickness series $\left(a-\mathrm{SiO}_{2}\right.$ respectively $\left.\mathrm{Sb}_{2} \mathrm{Te}_{3}\right)$ which revealed the contribution of the insulation layer respectively of the thermal boundary resistances. This enabled high-precision measurements of cross-plane thermal conductivities.

The results for the Kapitza resistances presented in Publication 1, show a thermal conductance $G$ associated with Kapitza $A\left(\mathrm{Pt} / \mathrm{a}-\mathrm{Si}_{2}+\mathrm{a}-\mathrm{Si}_{2} / \alpha-\mathrm{Al}_{2} \mathrm{O}_{3}\right)$ of $G_{\text {Kap A }} \approx$ $4 \times 10^{7}-1.31 \times 10^{8} \frac{\mathrm{W}}{\mathrm{m}^{2} \mathrm{~K}}$ which increases with increasing temperature. For the thermal conductance associated with Kapitza $B\left(\mathrm{Pt} / \mathrm{a}-\mathrm{Si}_{2}+\mathrm{a}-\mathrm{Si}_{2} / \mathrm{Sb}_{2} \mathrm{Te}_{3}+\mathrm{Sb}_{2} \mathrm{Te}_{3} / \alpha-\mathrm{Al}_{2} \mathrm{O}_{3}\right)$ no clear temperature trend was observed. The average value was determined to be $G_{\text {Kap B }} \approx$ $9 \times 10^{6} \frac{\mathrm{W}}{\mathrm{m}^{2} \mathrm{~K}}$ close to the very low value of $G=8 \times 10^{6} \frac{\mathrm{W}}{\mathrm{m}^{2} \mathrm{~K}}$ reported by ref. [98] for a $\mathrm{Bi} /$ Diamond interface.

One has to note that Kapitza $B$ includes 3 interfaces, from which the $\mathrm{Pt} / \mathrm{a}-\mathrm{SiO}_{2}$ can be assumed to have a relatively low single Kapitza resistance, since it is also part of Kapitza $A$ which is significantly lower compared to Kapitza B. For that reason, the interfaces a- $\mathrm{SiO}_{2} / \mathrm{Sb}_{2} \mathrm{Te}_{3}$ and $\mathrm{Sb}_{2} \mathrm{Te}_{3} / \alpha-\mathrm{Al}_{2} \mathrm{O}_{3}$ most likely put the main part of Kapitza B. In both cases heat transport mechanisms vary (insulating a-SiO $\mathrm{S}_{2}$ and $\alpha-\mathrm{Al}_{2} \mathrm{O}_{3}$ vs. considerable electronic conductivity in $\mathrm{Sb}_{2} \mathrm{Te}_{3}$ ) which usually contributes to large thermal boundary resistances.

Considering lattice thermal conductivity, the materials show different Debye temperatures $\left(\mathrm{Sb}_{2} \mathrm{Te}_{3}\right.$ : $165 \mathrm{~K}$ [69], amorphous a-SiO 2 : 93 - $198 \mathrm{~K}$ (depending on "grain" sizes) [136], $\alpha-\mathrm{Al}_{2} \mathrm{O}_{3}: 1042 \mathrm{~K}$ [95]). Different Debye temperatures commonly are associated with acoustic mismatches between of the respective materials which means that many phonon frequencies present in one materials are not allowed in the other material (although the application of the phonon model is limited for amorphous materials). As a consequence, thermal transfer is impeded and a temperature gradient arises at the interface. Only considering the difference in Debye temperatures, it can be assumed that the Kapitza resistance at the interface $\mathrm{Sb}_{2} \mathrm{Te}_{3} / \alpha-\mathrm{Al}_{2} \mathrm{O}_{3}$ clearly is the largest of the three interface resistances included in Kapitza $B$. It can be concluded that it is smaller but of the same order as the interface resistance of $\mathrm{Bi} /$ Diamond. This matches the expectation, since the Debye temperature difference of the latter is even higher.

In conclusion, the Kapitza resistances at thin film thermal conductivity measurement configurations of $\mathrm{Sb}_{2} \mathrm{Te}_{3}$ turned out to not being negligible. Presumably this applies also 
for other Tetradymite and VdW layered materials. The huge Kapitza resistances also have to be taken into account considering thin film thermoelectric applications.

$\mathrm{Sb}_{2} \mathrm{Te}_{3} / \alpha-\mathrm{Al}_{2} \mathrm{O}_{3}$ interface: HAADF and EELS. The interface film/substrate has been carefully studied by HRTEM, STEM HAADF and EELS. On top of the substrate, a quintuple layer was observed exhibiting a different contrast compared to the subsequent quintuple layers. Possible explanations are a higher degree of disorder in this layer, a stronger thinning at the last FIB steps and a different chemical composition. EELS analysis showed a slightly lower Te content and the presence of oxygen which might explain the contrast deviation, however, quantitative EELS analysis is limited due to overlaps of Sb M edge, O K edge and Te M edge.

The irregularity at the interface concerning structure and composition might significantly contribute to the large Kapitza resistance by creating de facto two interfaces and further enhancing diffuse scattering. This further strengthens the assumption that the interface $\mathrm{Sb}_{2} \mathrm{Te}_{3} / \alpha-\mathrm{Al}_{2} \mathrm{O}_{3}$ is the one with the highest Kapitza resistance within Kapitza $B$, if not the dominant one.

Low intrinsic $\kappa_{c}$ in $\mathbf{S b}_{2} \mathbf{T e}_{3}$. In order to determine the efficiency of thermoelectric devices, the $Z T$ value has to be known over the whole relevant temperature range. Van der Waals layered thermoelectrics in single crystalline form are often grown along the c-axis, so this should be the preferred measurement direction. Applying the advanced $3 \omega$ method and considering the determined Kapitza resistance and insulating layer contributions, the c-axis thermal conductivity of $\mathrm{Sb}_{2} \mathrm{Te}_{3}$ films with different thicknesses has been measured precisely over a huge temperature range. For the majority of the temperature range the results matched very well.

HRTEM and STEM revealed very low concentrations of planar defects in the films. This oberservation combined with the comparison with a room temperature literature value of $\kappa_{c}=1.65 \pm 0.11$ [67], being slightly lower than the own values, justifies the statement of having determined the intrinsic c-axis thermal conductivity of $\mathrm{Sb}_{2} \mathrm{Te}_{3}$.

Since nanostructuring and micro and nanocrystalline samples are widely assumed as promising approach aiming at high figure of merits, the comparison with polycrystalline samples [77] seemed to be advisable. It revealed that the thermal conductivity decreases with decreasing grain sizes (129 vs. $88 \mathrm{~nm}$ ) and except for low temperatures the values were close to the c-axis values. The apparent effect of grain boundaries of lowering thermal conductivity seems to be compensated by the fact that in polycrystalline materials also the higher a,b-plane thermal conductivity is contributing. As long as there is no clear proof that grain boundaries significantly increase electrical conductivity in $\mathrm{Sb}_{2} \mathrm{Te}_{3}$, no breakthrough can be expected on the polycrystalline route towards high $Z T$ values. 


\section{Chapter 5: Publication 2}

Thermal conductivity measurements of $\left(\mathrm{Sb}_{1-\mathrm{x}} \mathrm{Bi}_{\mathrm{x}}\right)_{2} \mathrm{Te}_{3}$ films and thereof derived $\kappa_{c, l}$ values have been complemented by lattice thermal conductivity calculations. The shown results reveal information about the influence of Umklapp respectively Rayleigh scattering on the c-axis lattice thermal conductivity. Bi content dependent strength of both scattering mechanisms is presented culminating in the evidence of a phonon glass state present in the highest doped solid solution sample.

Epitaxial, almost single crystalline solid solution films with low surface roughness and low defect concentrations. As shown in Publication 1 for the binary compound, XRD investigations and SEM topological analysis revealed (001) oriented Bi doped films with smooth surfaces. This was confirmed by HRTEM and STEM showing highly regular layered growth. The substrate-film interfaces resembled those at $\mathrm{Sb}_{2} \mathrm{Te}_{3}$ (Publication 1). The planar defect concentration emerged to be low, only slightly increasing with increasing $\mathrm{Bi}$ concentration and being stronger increased in the upper part of the $\mathrm{Bi}_{2} \mathrm{Te}_{3}$ film. In total, the films showed a high crystalline quality and comparability. Deviations at the $\mathrm{Bi}_{2} \mathrm{Te}_{3}$ film can be explained with different synthesis parameters since for $\mathrm{Bi}_{2} \mathrm{Te}_{3}$ a lower chamber pressure turned out to be necessary in order to achieve low surface roughness.

Uniform distribution of $\mathrm{Bi}$ atoms on the Pnictogen sites in the solid solution films. Comprehensive High Resolution STEM HAADF and SAED investigations performed for different $\mathrm{Bi}$ concentrations further revealed details about the spacial distribution of the pnictogens. Comparison of experimental and simulated HAADF images proved the presence of solid solutions for all ternary samples, without significant accumulation or depletion of $\mathrm{Bi}$ atoms. This represents a crucial finding regarding subsequent statements about structure property correlations. Worth mentioning is that two lamellas cut perpendicular to each other out of the same sample $(x=0.24)$ at different positions showed zone axes vertically standing to each other, strongly indicating a consistent crystalline orientation across the film.

Low lattice thermal conductivity due to Bi doping. For the $3 \omega$ measurements the already developed measurement geometry and procedures including Kapitza resistance data have been used. The $3 \omega$ investigations revealed temperature and $\mathrm{Bi}$ concentration dependent thermal conductivity progressions along the c-axis. To the best knowledge of the author this is the first time such comprehensive data on highly crystalline (001) oriented $\left(\mathrm{Sb}_{1-\mathrm{x}} \mathrm{Bi}_{\mathrm{x}}\right)_{2} \mathrm{Te}_{3}$ films including High Resolution TEM has been published.

Subtraction of $\kappa_{c, e}(T)$ (using literature data) revealed very similar lattice thermal conductivity values for $\mathrm{Sb}_{2} \mathrm{Te}_{3}$ and $\mathrm{Bi}_{2} \mathrm{Te}_{3}$, but a steeper increase heading towards lower temperatures for the $\mathrm{Sb}_{2} \mathrm{Te}_{3}$ sample.

For the solid solution samples, a clear trend of strongly decreasing $\kappa_{c}$ and $\kappa_{c, l}$ with increasing Bi content was observed. 
For $\mathrm{Bi}_{2} \mathrm{Te}_{3}$ the measured value at $300 \mathrm{~K}$ of $\kappa_{c}=1.27 \frac{\mathrm{W}}{\mathrm{mK}}$ well agrees with the reported value of $1.2 \pm 0.2 \frac{\mathrm{W}}{\mathrm{mK}}$ obtained by TDTR measurements at thin films [68].

Impact of Rayleigh and Umklapp scattering. Beyond experimental investigations and measurements, an important part of this publication is the numerical calculation of the lattice thermal conductivities along the c-axis. The author of this thesis has developed a computational routine using the program MATLAB (MathWorks ${ }^{\circledR}$ ) to obtain accurate phonon mode, frequency and temperature dependent $\kappa_{c, l}$ values taking into account scattering rates scaled by fit parameters. Specific materials data based on extensive literature research have been used. A good confirmation of the acoustic phonon dispersion relations are average acoustic phonon velocities being in good agreement with macroscopic determined sound velocities both for $\mathrm{Sb}_{2} \mathrm{Te}_{3}$ and for $\mathrm{Bi}_{2} \mathrm{Te}_{3}$.

Fitting the calculated results to experimental results (after subtraction of $\kappa_{c, e}(T)$ data from literature) yielded sample specific scattering parameters indicating an increasing Rayleigh scattering and Umklapp scattering with increasing Bi concentrations. Increasing Rayleigh scattering with increasing number of "0 dimensional" scattering centers might seem to be trivial, however, the respective parameter $B^{-1}$ (expressing the scattering strength) was increased by a factor of almost 27 just between the $\mathrm{Sb}_{2} \mathrm{Te}_{3}$ and the $\left(\mathrm{Sb}_{0.93} \mathrm{Bi}_{0.07}\right)_{2} \mathrm{Te}_{3}$ sample.

The increased Umklapp scattering might be connected with higher anharmonicity due to changes in chemical bonds. Also increased transition probabilites of three phonon Umklapp processes may play a role, however, at present, further dispersion relations simulations in the respective doping range are required to check or confirm this presumption. The assumption of high anharmonicity furthermore is supported by smeared reflexes in the SAED pattern of the $\mathrm{x}=0.24$ sample compared to that of the $\mathrm{x}=0$ sample.

Concerning the binary compounds, Umklapp scattering turned out to be a bit less pronounced in the $\mathrm{Bi}_{2} \mathrm{Te}_{3}$ sample. This finding is in good agreement with a slightly lower Grüneisen parameter (indicating the strength of anharmonicity) of 1.5 found for $\mathrm{Bi}_{2} \mathrm{Te}_{3}$ in comparison to 1.7 for $\mathrm{Sb}_{2} \mathrm{Te}_{3}$ [81]. Looking more closely to the lattice parameter $c$ which alters only marginally between the compounds $[58,59]$ reveals that the increased distances $\mathrm{Te}(1)-\mathrm{Bi}$ and $\mathrm{Te}(2)-\mathrm{Bi}$ come along with van der Waals gaps of decreased size. It can be assumed that this convergence of atomic distances and therefore atomic force constants may be linked with decreased anharmonicity and lower Umklapp scattering rates.

The pronounced Rayleigh scattering found for the $\mathrm{Bi}_{2} \mathrm{Te}_{3}$ sample reflects the significantly weaker decrease of $\kappa_{c, l}(T)$ in comparison with the other samples (partially except for $\mathrm{x}=0.24$, which will discussed separately). Conceivable is a connection between the observed relatively high planar defect concentrations in the $\mathrm{Bi}_{2} \mathrm{Te}_{3}$ sample and the weaker decrease in $\kappa_{c, l}(T)$. Furthermore, $\mathrm{Bi}_{2} \mathrm{Te}_{3}$ shows a high density of states especially for low frequency acoustic phonons which are rather weakly affected by Rayleigh scattering and a necessarily high parameter $B^{-1}$ might result from that fact. This sample additionally has been evaluated using other literature data than for the rest of the samples and small deviations might quickly have a strong effect. Besides, the applicability of 
the used Debye model is maybe different for $\mathrm{Sb}_{2} \mathrm{Te}_{3}$ and $\mathrm{Bi}_{2} \mathrm{Te}_{3}$. For that reasons, the comparability of $B$ for $\mathrm{x}=1$ and $B$ for $\mathrm{x}<1$ might be limited, which should be kept in mind.

The higher anharmonicity and the lower density of states at low phonon frequencies (stronger influence of doping by more pronounced Rayleigh scattering) on the Sb-rich side might be the reason for the minimum $\kappa_{c, l}$ being located in that half of the quasibinary phase diagram [5]. For that reason it seems recommendable to focus on this half when aiming at low lattice thermal conductivities.

Phonon glass - electron crystal. One highlight of this publication constitutes the finding of a constant value for $\kappa_{c, l}(T)$ derived from the $3 \omega$ measurements over a huge temperature range $(T \gtrsim 160 K)$ for a sample where approximately every fourth $\mathrm{Sb}$ atom has been substituted by a Bi atom. This so called Phonon glass - electron crystal behavior is a very desired state in thermoelectric materials research. The calculated phonon mean free paths of this sample only for low frequencies exceed the respective phonon wavelengths.

At $300 \mathrm{~K}$, the determined lattice thermal conductivity of the $\mathrm{x}=0.24$ sample is $0.42 \frac{\mathrm{W}}{\mathrm{m} \mathrm{K}}$. Crystallinity of the $\mathrm{x}=0.24$ sample could clearly be validated by XRD, HRTEM, STEM and SAED. At low temperatures, a typical "Umklapp scattering drop" is observed, indicating the crystalline nature of the sample. The presence of the phonon glass state as an intrinsic material property is an essential recognition which can be interpreted as being a result of extraordinary strong Umklapp scattering in combination with other effects such as strong Rayleigh scattering. This state might be one reason for high $Z T$ values reached at about $\mathrm{x}=0.25[23]$.

The discovery of a Phonon glass - electron crystal state encourages further research on "non nanostructured" single crystalline films with an emphasis on further doping targeting higher Seebeck coefficients and electronic conductivities while simultaneously maintaining the phonon glass state.

\section{Appendix 1: Publication 3}

The MOCVD deposition of $\mathrm{Bi}_{2} \mathrm{Te}_{3}$ thin films on Si substrates led to films with different crystalline orientations at the surface, which was shown by XRD. The polycrystalline nature of the samples was also observed via SEM.

HRTEM investigation carried out by the author was able to show regular growth of $\mathrm{Bi}_{2} \mathrm{Te}_{3}$ along the c-axis. Interestingly, at a certain distance to the substrate, the formation of differently oriented grains could be identified. Presumably due to a temperature gradient in the poor conducting films, the conditions for film growth along the c-axis worsen with increasing distance to the substrate. A possible approach tackling this problem might be a continuous increase of the heater temperature during the deposition process.

This link only could be established by HRTEM, since other, more "macroscopic", techniques fail. For example, SEM only determines topological information and XRD 
provides average crystal data of a huge area.

Due to the high surface roughness of the obtained films, proper insulation for the $3 \omega$ measurement was not feasible.

These results gave rise to switch to a thermal evaporation method using $\alpha-\mathrm{Al}_{2} \mathrm{O}_{3}$ subtrates. This switch turned out to be very successful, as can be seen at the results presented in Publication 1 and Publication 2.

\section{Appendix 2: Publication 4}

Difficulties concerning the MOCVD growth of highly textured Tetradymite films (as illustrated in Publication 3) resulted in turning towards the PVD process of thermal co-evaporation. Publication 4 presents results on PVD grown $\mathrm{Sb}_{2} \mathrm{Te}_{3}$ films of different thicknesses.

The author contributed HRTEM images of lamellas cut out of different films, thermal conductivity and TEM results on such films have been presented in Publication 1. Epitaxial growth of almost single crystalline and very regular films has been confirmed for different thicknesses. Density of planar defects has been determined to be very low. This publication demonstrates that thermal evaporation works well for synthesizing high quality thermoelectric $\mathrm{Sb}_{2} \mathrm{Te}_{3}$ films.

\section{Outlook}

The present thesis presents doping and temperature dependent thermal transport properties of the ternary material system $\left(\mathrm{Sb}_{1-\mathrm{x}} \mathrm{Bi}_{\mathrm{x}}\right)_{2} \mathrm{Te}_{3}$, obtained at thin film samples of high crystalline quality. For further improving the significance of the results, electrical conductivity measurements along the c-axis seem to be recommendable, in order to obtain precise $\kappa_{c, e}(x, T)$ values. Therefore deposition on a thin film of high electrical conductance or on an electrically conductive substrate (e.g. Nb doped $\mathrm{SrTiO}_{3}$ ) might be good options. The latter would also allow for $3 \omega$ measurements far below $100 \mathrm{~K}$ due to its relatively low thermal conductivity. Since $\mathrm{Sb}_{2} \mathrm{Te}_{3}$ and $\mathrm{Bi}_{2} \mathrm{Te}_{3}$ have been identified as being topological insulators, electrical measurements should be conducted at nanostructured pillars of varying diameters for separating topological from bulk contributions.

Further investigations on the $\left(\mathrm{Sb}_{1-\mathrm{x}} \mathrm{Bi}_{\mathrm{x}}\right)_{2} \mathrm{Te}_{3}$ system generating a $T$ vs. $\mathrm{x}$ diagram which indicates the extension of the Phonon glass - electron crystal state would provide additional valuable information about the thermal properties of the system. Also investigating the impact of other doping elements (e.g. substitution of Te by Se) might be very interesting in that context, in particular combined with measurements of Seebeck coefficients and electrical conductivities.

Last but not least, in order to determine actual $Z T$ values for possible TEG applica- 
tions, $\kappa_{c}$ measurements at higher temperatures are advisable, without however missing to characterize the structural changes taking place due to heat exposure. 


\section{Author contributions}

This thesis including appendices comprises four publications:

1. Publication 1: F. Rieger, K. Kaiser, G. Bendt, V. Roddatis, P. Thiessen, S. Schulz, and C. Jooss. „Low intrinsic c-axis thermal conductivity in PVD grown epitaxial $\mathrm{Sb}_{2} \mathrm{Te}_{3}$ films". Journal of Applied Physics 123:175108, 2018; DOI: 10.1063/1.5025491. [84]

2. Publication 2: F. Rieger, V. Roddatis, K. Kaiser, G. Bendt, S. Schulz, and C. Jooss. "Transition into a phonon glass in crystalline thermoelectric $\left(\mathrm{Sb}_{1-\mathrm{x}} \mathrm{Bi}_{\mathrm{x}}\right)_{2} \mathrm{Te}_{3}$ films". (to be published)

3. Publication 3: G. Bendt, S. Gassa, F. Rieger, C. Jooss, and S. Schulz. „Low-temperature MOCVD deposition of $\mathrm{Bi}_{2} \mathrm{Te}_{3}$ thin films using $\mathrm{Et}_{2} \mathrm{BiTeEt}$ as single source precursor". Journal of Crystal Growth 490:77-83, 2018; DOI: 10.1016/j.jcrysgro.2018.03.021. [137]

4. Publication 4: G. Bendt, K. Kaiser, A. Heckel, F. Rieger, D. Oing, A. Lorke, N. Perez Rodriguez, G. Schierning, C. Jooss, and S. Schulz. „Structural and thermoelectrical characterization of epitaxial $\mathrm{Sb}_{2} \mathrm{Te}_{3}$ high quality thin films grown by thermal evaporation". Semiconductor Science and Technology 33:105002, 2018; DOI: 10.1088/1361-6641/aad7a3. [110]

Publications 1, 3 and 4 have been published in peer-reviewed journals. Publication 2 is ready for submission to a peer-reviewed journal. All publications present original research of the author of this thesis (below referred to as "the author").

Hereinafter, the author contributions to the different articles are listed.

In general, TEM investigations and thermal conductivity measurements have been performed by the author at the Institute of Material Physics at the University of Göttingen. Persons listed are or have been members of the IMP, except when otherwise specified. The development of the appropriate FIB procedure for the lamellas has been done by the author, assisted by Dr. Vladimir Roddatis and Volker Raddisch. HRTEM and HAADF STEM investigations have been carried out by the author in collaboration with Dr. Vladimir Roddatis. The resist masks for the $3 \omega$ heating structures have been applied by electron beam lithography by Mike Kanbach. Film thickness measurements 
by profilometry have been done by the author.

The synthesis of films and partially their characterization have been done at the Institute of Inorganic Chemistry and Center for Nanointegration Duisburg-Essen (Cenide) at the University of Duisburg-Essen in the group of Prof. Dr. Stephan Schulz (Kevin Kaiser, Dr. Georg Bendt) respectively in the group of Prof. Dr. Matthias Epple (Dietrich Tönnes).

Publication 1. Preparation of the $\mathrm{Sb}_{2} \mathrm{Te}_{3}$ films as well as XPS characterization and evaluation have been done by Kevin Kaiser. XRD has been conducted experimentally by Dr. Georg Bendt respectively Dietrich Tönnes and analyzed by Kevin Kaiser. The published topological investigations by SEM have been done by the author. The $3 \omega$ method development has been done by the author in collaboration with Patrick Thiessen during his Master thesis. The development of an ion beam sputtering deposition routine for insulation layers has been done by the author. The insulation layer thickness series as well as the $\mathrm{Sb}_{2} \mathrm{Te}_{3}$ layer thickness series have been conceived, executed and analyzed by the author. The sample geometry presented in this publication has been conceived by the author. Ion beam sputtering of the insulation layers and Pt as well as lift out process and electrical testing of the insulation layers have been carried out by the author. $3 \omega$ measurement and evaluation have been done by the author. TEM lamella preparation via FIB and precharacterization have been done by the author, while HRTEM, STEM, HAADF, SAED and EELS investigations have been carried out by the author partially in collaboration with Dr. Vladimir Roddatis. EELS analysis has been done by the author with the support of Prof. Dr. Christian Jooß. $3 \omega$ analysis concerning mean free paths has been developed by the author in consultation with Prof. Dr. Christian Jooß. Figures (except figures 1 and S1, which have been prepared in the group of Prof. Dr. Stephan Schulz) and manuscript text have been developed by the author, Dr. Vladimir Roddatis and Prof. Dr. Christian Jooß. Patrick Thiessen contributed to the methodological part in the Supplemental Information. The sections of the manuscript with respect to synthesis and XRD have been developed by Dr. Georg Bendt, Kevin Kaiser and Stephan Schulz.

Publication 2. Synthesis of the $\left(\mathrm{Sb}_{1-\mathrm{x}} \mathrm{Bi}_{\mathrm{x}}\right)_{2} \mathrm{Te}_{3}$ films has been carried out by Kevin Kaiser. XRD has been performed experimentally by Dr. Georg Bendt respectively Dietrich Tönnes and analyzed by Kevin Kaiser. EDX has been carried out and analyzed by Kevin Kaiser and the author. Topological analysis by SEM has been conducted by the author. $3 \omega$ measurements and the preceding sputtering of insulation layers and Pt heating structures, including lift out and electrical testing, have been done by the author. The subsequent determination of lattice thermal conductivities by subtraction of electrical contributions, based on literature data, has been done by the author. Drawing up and writing of the script for lattice thermal conductivity calculations and on this basis the analysis for the different samples have been done by the author in consultation with Prof. Dr. Christian Jooß. TEM lamellas have been prepared by the author via FIB. HRTEM, STEM, HAADF and SAED investigations have been carried out by the author in collaboration with Dr. Vladimir Roddatis. HAADF image simulations have been done by Dr. Vladimir Roddatis. The figures have been prepared by the author in 
consultation with Prof. Dr. Christian Jooß and Dr. Vladimir Roddatis, the latter was strongly involved in creating the figures 1, 2, S3, S4, S5 and S6. For preparing figure $\mathrm{S} 1$, the author used XRD data obtained from Kevin Kaiser. The manuscript text was developed by the author, Prof. Dr. Christian Jooß and Dr. Vladimir Roddatis. The sections of the manuscript concerning synthesis and XRD have been developed by Kevin Kaiser, Dr. Georg Bendt and Prof. Dr. Stephan Schulz.

The author contributed to Publication 3 and Publication 4. The main focus of these publications is on film growth and different physical quantities in dependence of deposition method, substrate and growth parameters. Film growth for Publication 3 has been done by Sanae Gassa in consultation with Dr. Georg Bendt, while films shown in Publication 4 have been synthesized by Alla Heckel and Kevin Kaiser. XRD measurement has been conducted by Dr. Georg Bendt and Dietrich Tönnes and the XRD analysis has been done by Dr. Georg Bendt for Publication 3 and by Alla Heckel and Kevin Kaiser for Publication 4. Alla Heckel has done the SEM and EDX analysis for Publication 4. Nicolas Perez Rodriguez in consultation with Dr. Gabi Schiernig (both IFW Dresden) has carried out the thermoelectric measurements shown in Publication 4. For Publication 4, Dennis Oing in consultation with Prof. Dr. Axel Lorke (both Faculty of Physics and Center for Nanointegration Duisburg-Essen (CENIDE), University of Duisburg-Essen) has performed four-point and Hall measurements.

For both publications, the author prepared the TEM lamellas via FIB and did precharacterizations. HRTEM and SAED have been performed by the author in collaboration with Dr. Vladimir Roddatis. The respective figures, figure 6 in Publication 3 and figure 10 in Publication 4, have been created and composed by the author in consultation with Prof. Dr. Christian Jooß. The respective (TEM) text passages in both publications have been developed by the author and Prof. Dr. Christian Jooß. 



\section{Appendix 1: Low-temperature MOCVD deposition of $\mathrm{Bi}_{2} \mathrm{Te}_{3}$ thin films using $\mathrm{Et}_{2} \mathrm{BiTeEt}$ as single source pre- cursor}

This chapter consists of the accepted manuscript of the original article

Low-temperature MOCVD deposition of $\mathrm{Bi}_{2} \mathrm{Te}_{3}$ thin films using $\mathrm{Et}_{2} \mathrm{BiTeEt}$ as single source precursor

G. Bendt ${ }^{1}$, S. Gassa ${ }^{1}$, F. Rieger ${ }^{2}$, C. Jooss ${ }^{2}$, and S. Schulz ${ }^{1}$

${ }^{1}$ Institute of Inorganic Chemistry and Center for Nanointegration Duisburg-Essen (Cenide), University of Duisburg - Essen, 45141 Essen, Germany

${ }^{2}$ Institute of Material Physics, University of Göttingen, 37077 Göttingen, Germany

Originally published in Journal of Crystal Growth 490:77-83, 2018. [137]

DOI: $10.1016 /$ j.jcrysgro.2018.03.021

This manuscript is published here in accordance with the Author Rights of Elsevier. 



\title{
Low-temperature MOCVD deposition of $\mathrm{Bi}_{2} \mathrm{Te}_{3}$ thin films using $\mathrm{Et}_{2} \mathrm{BiTeEt}$ as Single Source Precursor.
}

\author{
Georg Bendt ${ }^{1}$, Sanae Gassa ${ }^{1}$, Felix Rieger ${ }^{2}$, Christian Jooss ${ }^{2}$ and Stephan Schulz ${ }^{1}$
}

${ }^{1}$ Institute of Inorganic Chemistry and Center for Nanointegration Duisburg-Essen (CENIDE), University of Duisburg-Essen, Universitätsstr. 5-7, D-45117 Essen, Germany. Fax: 4902011833830 ; Tel: 490201 1834635; E-mail: stephan.schulz@uni-due.de

${ }^{2}$ Institute for Materials Physics, University of Göttingen, Friedrich-Hund-Platz 1, D-37077 Göttingen, Germany.

\begin{abstract}
$\mathrm{Et}_{2} \mathrm{BiTeEt}$ was used as single source precursor for the deposition of $\mathrm{Bi}_{2} \mathrm{Te}_{3}$ thin films on $\mathrm{Si}(100)$ substrates by metal organic chemical vapor deposition (MOCVD) at very low substrate temperatures. Stoichiometric and crystalline $\mathrm{Bi}_{2} \mathrm{Te}_{3}$ films were grown at $230{ }^{\circ} \mathrm{C}$, which is approximately $100^{\circ} \mathrm{C}$ lower compared to conventional MOCVD processes using one metal organic precursors for each element. The $\mathrm{Bi}_{2} \mathrm{Te}_{3}$ films were characterized using scanning electron microscopy, high-resolution transmission electron microscopy and X-ray diffraction. The elemental composition of the films, which was determined by energy-dispersive X-ray spectroscopy and Xray photoelectron spectroscopy, was found to be strongly dependent of the substrate temperature.
\end{abstract}

Keywords. A3. Metalorganic chemical vapor deposition, B1. Bismuth compounds, B1. Tellurides, B1. Nanomaterials, B2. Semiconducting materials 


\section{Introduction}

Thermoelectric materials allow the direct conversion of heat into electrical power without the use of mechanic transmission. Despite the inefficiency of thermoelectric materials for large scale energy generation ${ }^{[1]}$, these materials are ideal for harvesting energy for sensors and other small devices. The thermoelectric efficiency of a material is given by the dimensionless figure of merit $Z T$ $=\left(S^{2} \sigma / \kappa\right) T(S=$ Seebeck coefficient, $\sigma=$ specific electrical conductivity, $\kappa=$ thermal conductivity $=$ sum of electronic $\kappa_{\mathrm{el}}$ and lattice $\kappa_{\mathrm{la}}$ contributions, $\mathrm{T}=$ absolute temperature $\left.[\mathrm{K}]\right)^{[2]}$. $\mathrm{Bi}_{2} \mathrm{Te}_{3}$ combines a high Seebeck coefficient and high electrical conductivity with a glass-like low thermal conductivity, resulting in a superior thermoelectric performance near room temperature. However, the synthesis of high-quality $\mathrm{Bi}_{2} \mathrm{Te}_{3}$ materials such as nanoparticles and thin films is very challenging, especially due to the high tendency of $\mathrm{Bi}_{2} \mathrm{Te}_{3}$ to form antisite defects. In particular, the incorporation of excess bismuth into the crystal lattice and subsequent formation of sandwichlike structures of the general form $\left(\mathrm{Bi}_{2}\right)_{n}\left(\mathrm{Bi}_{2} \mathrm{Te}_{3}\right)_{\mathrm{m}}$ are generally observed for tetradymite-type materials $^{[3]}$.

Metal organic chemical vapor deposition (MOCVD) is a widely used industrial process for the deposition of $\mathrm{Bi}_{2} \mathrm{Te}_{3}$ films, in which metal organic compounds such as trialkyl bismuth $\left(\mathrm{R}_{3} \mathrm{Bi}\right)$ and dialkyl tellurium $\left(\mathrm{R}_{2} \mathrm{Te}\right)$ are typical precursors. For example, Giani et al. reported on the growth of $\mathrm{Bi}_{2} \mathrm{Te}_{3}$ films using trimethyl bismuth ( $\left.\mathrm{Me}_{3} \mathrm{Bi}, \mathrm{TMBi}\right)$ and diethyl tellurium $\left(\mathrm{Et}_{2} \mathrm{Te}, \mathrm{DETe}\right)$ at $450{ }^{\circ} \mathrm{C}$ on pyrex and silicon substrates ${ }^{[4]}$, while You et al. successfully grew $\mathrm{Bi}_{2} \mathrm{Te}_{3}$ thin films using $\mathrm{TMBi}$ and diisopropyl tellurium (i- $\operatorname{Pr}_{2} \mathrm{Te}$, DIPTe) at $400{ }^{\circ} \mathrm{C}$ on GaAs(001) substrates ${ }^{[5]}$. Alternative tellurium precursors such as diethyl ditelluride $\left(\mathrm{Et}_{2} \mathrm{Te}_{2}, \mathrm{DEDTe}\right)$ and triethyl bismuth $\left(\mathrm{Et}_{3} \mathrm{Bi}, \mathrm{TEBi}\right)$ were also used for the deposition of highly c-oriented $\mathrm{Bi}_{2} \mathrm{Te}_{3}$ on $\mathrm{Al}_{2} \mathrm{O}_{3}(0001)$ at $400{ }^{\circ} \mathrm{C}^{[6]}$. To the best of our knowledge, thin film deposition at lower substrate temperatures $\left(300{ }^{\circ} \mathrm{C}\right)$ was only achieved by Kang et al. using a combination of TEBi and di-tertiarybutyl tellurium $\left(t-\mathrm{Bu}_{2} \mathrm{Te}\right.$, DTbTe) on $\mathrm{SiO}_{2}$ substrates $^{[7]}$. For these specific types of tetradymite materials, lower substrate temperatures have several advantages including energy saving and lower processing costs, but most importantly, the formation of antisite defects - in this case, the replacement of Te atoms by Bi atoms - can be reduced. The formation of antisite defects strongly correlates with the synthetic conditions; its formation is favored at high temperature and low pressure conditions due to the high vapor pressure of elemental Te. Consequently, low deposition temperatures are advantageous for the synthesis of highly-stoichiometric $\mathrm{Bi}_{2} \mathrm{Te}_{3}$ materials. 
In addition to simple binary tetradymite-type films, the deposition of multilayer structures has been previously investigated. Venkatasubramanian et al. reported on record-high $Z T$ values of up to 2.4 at $300 \mathrm{~K}$ for p-type $\mathrm{Bi}_{2} \mathrm{Te}_{3} / \mathrm{Sb}_{2} \mathrm{Te}_{3}$ superlattice devices, which was ascribed to an effective control of the transport of phonons and electrons in the superlattices ${ }^{[8]}$. However, such high $Z T$ values have never been reproduced by other groups, thus the applicability of this concept was questioned ${ }^{[9]}$. In particular the low thermal stability of $\mathrm{Bi}_{2} \mathrm{Te}_{3} / \mathrm{Sb}_{2} \mathrm{Te}_{3}$ superlattices was found to hamper their potential use in technical applications ${ }^{[10-12]}$.

Single source precursors (SSPs) have been shown to be very promising candidates for lowtemperature deposition processes. Chivers et al. reported on the deposition of $\mathrm{Sb}_{2} \mathrm{Te}_{3}$ nanoplates by aerosol assisted chemical vapor deposition (AACVD) using $\left[\mathrm{Sb}\left\{\left(\operatorname{TeP}^{\mathrm{i}} \mathrm{Pr}_{2}\right)_{2} \mathrm{~N}\right\}_{3}\right]^{[16]}$, while alkylchalcogenostibines $\mathrm{Ph}_{2} \mathrm{SbTeEt}$ and $\mathrm{MeSb}(\mathrm{TeBu})_{2}$ were used by Kim et al. and Reid et al. as SSP for the solution-based formation of $\mathrm{Sb}_{2} \mathrm{Te}_{3}$-nanoplates and the chemical vapor deposition of $\mathrm{Sb}_{2} \mathrm{Te}_{3}$ thin films, respectively ${ }^{[17-19]}$. We recently demonstrated that the thermal decomposition of the SSP $\left(\mathrm{Et}_{2} \mathrm{Sb}\right)_{2} \mathrm{Te}$ in solution-based ${ }^{[13,14]}$ and gas-phase-based processes ${ }^{[15]}$ allowed the generation of highly stoichiometric, crystalline $\mathrm{Sb}_{2} \mathrm{Te}_{3}$ nanoparticles and thin films at rather low temperatures $\left(180-200{ }^{\circ} \mathrm{C}\right)$. These nanomaterials exhibited very high Seebeck coefficients, indicating low concentrations of antisite defects.

So far, there has been no report on SSPs being successfully used in the deposition of $\mathrm{Bi}_{2} \mathrm{Te}_{3}$ thin films, or in the solution-based synthesis of $\mathrm{Bi}_{2} \mathrm{Te}_{3}$ nanoparticles. Despite the limited number of stable bismuth-tellurium containing molecules ${ }^{[20]}$, these compounds remain highly sensitive to air, moisture, light, and temperature. Attempts to investigate $\left(\mathrm{Et}_{2} \mathrm{Bi}\right)_{2} \mathrm{Te}$ and $\mathrm{Et}_{2} \mathrm{BiTeEt}$ as potential SSPs for the wet chemical synthesis of $\mathrm{Bi}_{2} \mathrm{Te}_{3}$ in organic solvents ${ }^{[21]}$ were not fruitful. The thermal decomposition of both precursors only resulted in the formation of $\mathrm{Bi}$-rich phases (i.e. $\mathrm{Bi}_{4} \mathrm{Te}_{3}$ and $\left.\mathrm{Bi}_{2} \mathrm{Te}\right)$, most likely due to the weak $\mathrm{Bi}-\mathrm{C}$ bond.

To the best of our knowledge, there has only been a single report on the successful use of a Bi-Te containing SSP by MOCVD methods; Reid et al. deposited high-quality $\mathrm{Bi}_{2} \mathrm{Te}_{3}$ thin films using the bismuth telluroether complex $\left[\mathrm{BiCl}_{3}\left(\mathrm{TeBu}_{2}\right)_{3}\right]^{[22]}$. Unfortunately, high substrate temperatures are required to achieve acceptable growth rates due to the strong $\mathrm{Bi}-\mathrm{Cl}$ bond, which is a severe drawback of this precursor. In addition, the use of chloride-containing precursors should generally be avoided due to their corrosive nature. 


\section{Experimental}

$\mathrm{Et}_{2} \mathrm{BiTeEt}^{[23-25]}$ was freshly prepared for each MOCVD study by mixing equimolar amounts of $\mathrm{Et}_{2} \mathrm{Te}_{2}$ and $\mathrm{Et}_{4} \mathrm{Bi}_{2}$ under argon atmosphere inside a glovebox. $\mathrm{Et}_{2} \mathrm{BiTeEt}$ was obtained as a red liquid via an exchange reaction (Scheme 1), typically occurring between dipnictogenes $R_{4} E_{2}(R=$ alkyl, aryl; $E=$ $\mathrm{Sb}, \mathrm{Bi})$ and dichalcogenanes $\mathrm{R}_{2} \mathrm{X}_{2}(\mathrm{R}=\text { alkyl, aryl; } \mathrm{X}=\mathrm{S}, \mathrm{Se}, \mathrm{Te})^{[23,24]}$.

Scheme 1. Synthesis of Et ${ }_{2} B i T e E t$.

$$
\mathrm{Et}_{2} \mathrm{Bi}-\mathrm{BiEt}_{2}+\mathrm{EtTe}-\mathrm{TeEt} \longrightarrow 2 \mathrm{Et}_{2} \mathrm{Bi}-\mathrm{TeEt}
$$

The thermal properties of $\mathrm{Et}_{2} \mathrm{BiTeEt}$ make this molecule very promising for MOCVD studies. According to our differential scanning calorimetry (DSC) study ${ }^{[21]}$, the thermal decomposition of $\mathrm{Et}_{2} \mathrm{BiTeEt}$ starts at $85^{\circ} \mathrm{C}$ with the formation of $\mathrm{Et}_{3} \mathrm{Bi}$ and $\mathrm{Et}_{2} \mathrm{Te}$, which were found to decompose at higher temperatures $\left(225{ }^{\circ} \mathrm{C}\right.$ and $270{ }^{\circ} \mathrm{C}$, respectively). Thermal decomposition of a solution of $\mathrm{Et}_{2} \mathrm{BiTeEt}$ in diisopropylbenzene at $100{ }^{\circ} \mathrm{C}$ only yielded bismuth-rich $\mathrm{Bi}_{2} \mathrm{Te}$ phase rather than the $\mathrm{Bi}_{2} \mathrm{Te}_{3}$ phase. The decomposition is most likely initiated with the homolytic cleavage of the Bi-Te bond and the subsequent formation of $\mathrm{Et}_{2} \mathrm{Bi}$ - and $\mathrm{EtTe}$ - radical species. These radicals react to give $\mathrm{Et}_{3} \mathrm{Bi}\left(3 \mathrm{Et}_{2} \mathrm{Bi} \cdot \rightarrow 2 \mathrm{Et}_{3} \mathrm{Bi}+\mathrm{Bi}\right)$ and $\mathrm{Et}_{2} \mathrm{Te}\left(2 \mathrm{EtTe} \cdot \rightarrow \mathrm{Et}_{2} \mathrm{Te}+\mathrm{Te}\right)$, along with elemental $\mathrm{Bi}$ and $\mathrm{Te}$, which ultimately form binary bismuth telluride phases.

MOCVD Deposition. MOCVD studies were performed in a hot-wall MOCVD reactor. $\mathrm{Bi}_{2} \mathrm{Te}_{3}$ films were deposited on $\mathrm{Si}(100)$ substrates at a working pressure of $10 \mathrm{mbar}$. $\mathrm{Si}(100)$ substrates were degreased with acetone, treated with hydrofluoric acid and heated to $500{ }^{\circ} \mathrm{C}$ at $10^{-3} \mathrm{mbar}$ for 1 hour in the reactor. Argon was used as carrier gas (40 sccm). The temperature and pressure of the precursor's glass bubbler were kept constant at $25^{\circ} \mathrm{C}$ and atmospheric pressure, respectively. Upon completion of the film deposition (growth time $=15$ minutes), the system was cooled to ambient temperature within 30 minutes.

X-ray photoelectron spectroscopy. XPS studies were performed using a Versaprobe IITM (Ulvac-Phi) with monochromatic Al $\mathrm{K}_{\alpha}$ light at $1486.6 \mathrm{eV}$ photon energy. The emission angle between analyzer and the sample was $45^{\circ}$. The $\mathrm{Cu} 2 \mathrm{p}$ signal at $932.67 \mathrm{eV}$ binding energy, which was obtained with a sputter cleaned $\mathrm{Cu}$ foil, was used as the binding energy reference. The foil and powder were adhered onto insulating double-sided tape, while charging effects were compensated with a dualbeam neutralizing approach using electrons and slow-moving argon ions.

Electron microscopy. Morphology and elemental composition of the films were analyzed by 
scanning electron microscopy (SEM) using a Jeol JSM 6510 microscope equipped with a Bruker Quantax 400 EDX spectrometer. The relative error for the EDX determination was calculated using the Esprit 4.0 software from Bruker.

HRTEM studies were conducted using a CS image corrected FEI TITAN with a lateral resolution limit of $0.08 \mathrm{~nm}$ at $300 \mathrm{kV}$. TEM lamellas were prepared in an FEI Nova Nanolab600 FIB. A $30 \mathrm{kV}$ accelerator voltage was used to cut the lamellas in the first thinning steps, while $5 \mathrm{kV}$ were applied for the last thinning step in order to reduce knock-on damage by the Ga ions.

X-ray powder diffraction. Grazing incidence X-ray powder diffraction (GIXRD) patterns were obtained at ambient temperature $\left(25 \pm 2^{\circ} \mathrm{C}\right)$ using a Panalytical Empyrean diffractometer with CuK $\alpha$ radiation $(\lambda=1.5418 \AA, 40 \mathrm{kV}, 40 \mathrm{~mA})$ and a fixed angle of incidence of $1^{\circ}$. The films were investigated in the range of $5-90^{\circ} 2 \Theta$ with a step size of $0.05^{\circ} 2 \Theta$.

\section{Results and Discussion}

Film deposition studies were performed at different substrate temperatures of $215^{\circ} \mathrm{C}, 230^{\circ} \mathrm{C}$ and $245^{\circ} \mathrm{C}$. The formation of dense films required a minimum substrate temperature of $230{ }^{\circ} \mathrm{C}$, while lower substrate temperature resulted in the growth of isolated $\mathrm{Bi}_{2} \mathrm{Te}_{3}$ platelets (Fig. 1). A dense film was also obtained at $245{ }^{\circ} \mathrm{C}$, but EDX studies revealed that this film does not show the expected 2:3 molar ratio ( $\mathrm{Bi}: \mathrm{Te}$ ) but rather a 4:3 molar ratio, pointing to the formation of Bi-rich $\mathrm{Bi}_{4} \mathrm{Te}_{3}$.

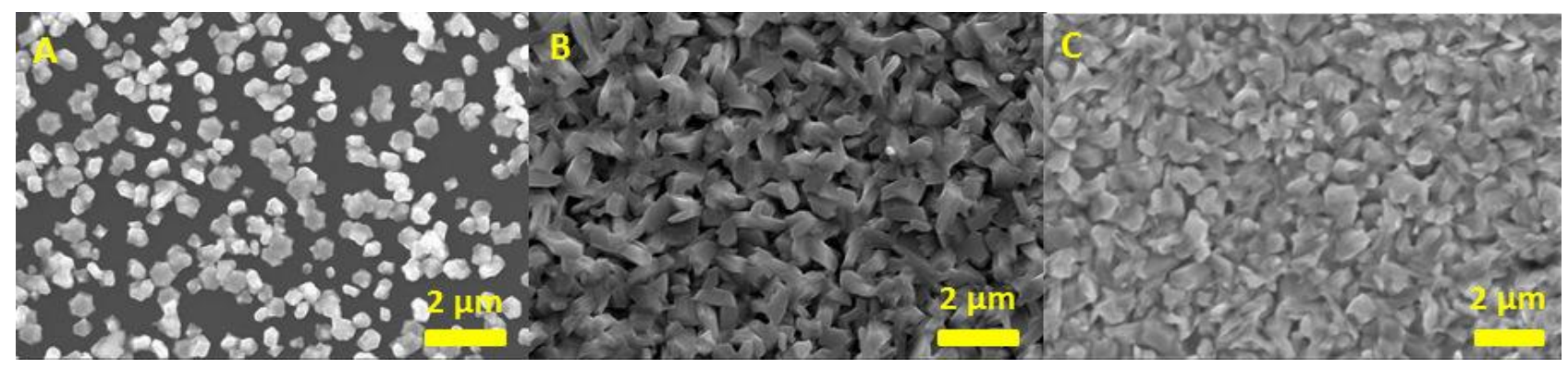

Figure 1. SEM picture of isolated $\mathrm{Bi}_{2} \mathrm{Te}_{3}$ hexagons grown at $215^{\circ} \mathrm{C}(\mathrm{A})$ and dense bismuth telluride films grown at $230^{\circ} \mathrm{C}(\mathrm{B})$ and $245^{\circ} \mathrm{C}(\mathrm{C})$.

The morphology of the silver-grey $\mathrm{Bi}_{2} \mathrm{Te}_{3}$ film deposited at $230{ }^{\circ} \mathrm{C}$ was investigated in detail by scanning electron microscopy (SEM). The images reveal that the dense film consists of intergrown $250 \mathrm{~nm}$ thick grains (Fig. 2). A cross section photograph of the film shown in Fig. 2C clearly indicates that the $\mathrm{Bi}_{2} \mathrm{Te}_{3}$ film is built with the grains laid or standing tilted on the surface of the substrate. 


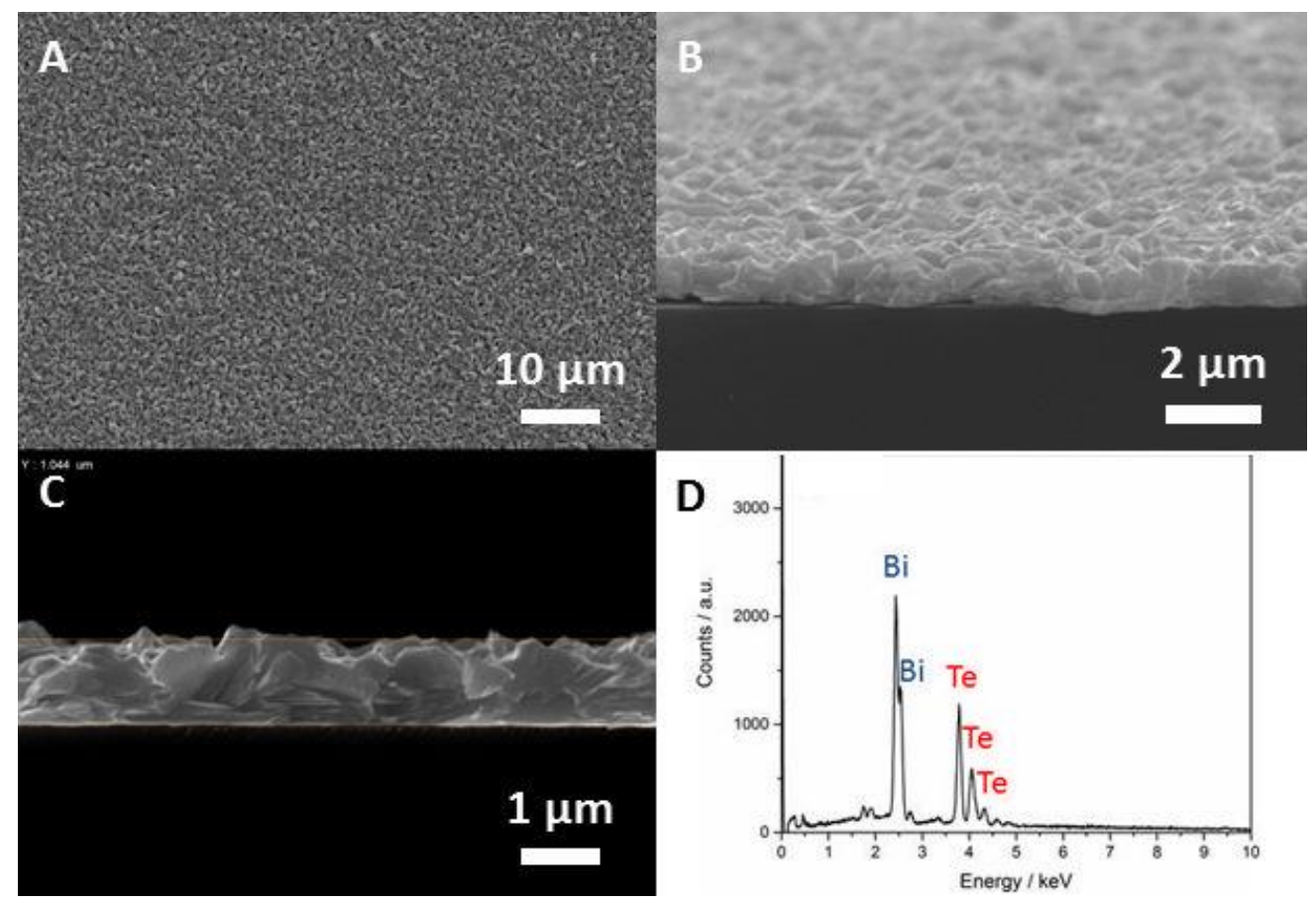

Figure 2. SEM images at different magnifications (A, B) and cross section photographs (C) of a $\mathrm{Bi}_{2} \mathrm{Te}_{3}$ film grown at $230{ }^{\circ} \mathrm{C}$ on a $\mathrm{Si}(100)$ substrate; EDX spectrum (D) of the $\mathrm{Bi}_{2} \mathrm{Te}_{3}$ film.

Energy-dispersive X-ray (EDX) spectra of the films show the characteristic peaks for bismuth at around $2.5 \mathrm{keV}$, and for tellurium at around $4.0 \mathrm{keV}$ (Fig. 2D). In addition, small peaks for carbon at $0.3 \mathrm{keV}$ and oxygen at $0.5 \mathrm{keV}$ were also observed. Quantitative elemental analysis reveals that the $\mathrm{Bi}$ :Te ratio of the films grown on $\mathrm{Si}(100)$ substrates strongly depends on the substrate temperature. Values close to the theoretical value for $\mathrm{Bi}_{2} \mathrm{Te}_{3}$ (40:60) were obtained for films grown at $215{ }^{\circ} \mathrm{C}$ and $230{ }^{\circ} \mathrm{C}$, whereas growth at $245^{\circ} \mathrm{C}$ yielded a Bi-rich film (Table 1 ).

Table 1. Elemental composition of bismuth telluride films grown at different substrate temperatures on $\mathrm{Si}(100)$ substrate as determined by EDX, and growth rates for the deposition of dense films at $230{ }^{\circ} \mathrm{C}$ and $245^{\circ} \mathrm{C}$.

\begin{tabular}{|c|c|c|c|}
\hline & $\mathbf{2 1 5}{ }^{\circ} \mathbf{C}$ & $\mathbf{2 3 0}{ }^{\circ} \mathbf{C}$ & $\mathbf{2 4 5}{ }^{\circ} \mathbf{C}$ \\
\hline Bi & $39.9 \pm 1.0^{[\mathrm{a}]}$ & $40.4 \pm 1.3$ & $48.4 \pm 0.9$ \\
\hline Te & $60.1 \pm 0.9$ & $59.6 \pm 1.1$ & $51.6 \pm 0.8$ \\
\hline Growth rate $\boldsymbol{\mu m} \cdot \mathbf{h}^{-1}$ & --- & 4.0 & 4.8 \\
\hline
\end{tabular}

${ }^{\text {[a] }}$ The EDX error was calculated using the software Esprit 4.0 from Bruker.

The crystallinity of the films was investigated by X-ray diffraction (XRD). The patterns were measured in grazing incidence geometry (GIXRD). All observed Bragg reflections can be indexed on the basis of rhombohedral $\mathrm{Bi}_{2} \mathrm{Te}_{3}$ (PDF card 15-0863) and $\mathrm{Bi}_{4} \mathrm{Te}_{3}$ (PDF card 33-216). Peaks indicating 
the formation of other bismuth telluride phases, oxidation products such as $\mathrm{Bi}_{2} \mathrm{O}_{3}, \mathrm{Sb}_{2} \mathrm{O}_{3}, \mathrm{TeO}_{2}$ or elemental Te were not detected.

The surface sensitive GIXRD pattern allows a clear phase identification of the deposited material films (Fig. 3). The GIXRD patterns obtained from the films deposited at $215^{\circ} \mathrm{C}$ and $230{ }^{\circ} \mathrm{C}$ can both be indexed on the basis of phase pure $\mathrm{Bi}_{2} \mathrm{Te}_{3}$, whereas the film deposited at $245{ }^{\circ} \mathrm{C}$ shows the presence of Bi-rich $\mathrm{Bi}_{4} \mathrm{Te}_{3}$ as secondary phase, confirming the EDX results. The differentiation between the two bismuth telluride phases is challenging since the structural differences are very small, and therefore resulting in similar XRD patterns. In this case, the different positions of the 006 reflections around $17.6^{\circ}$ were used.

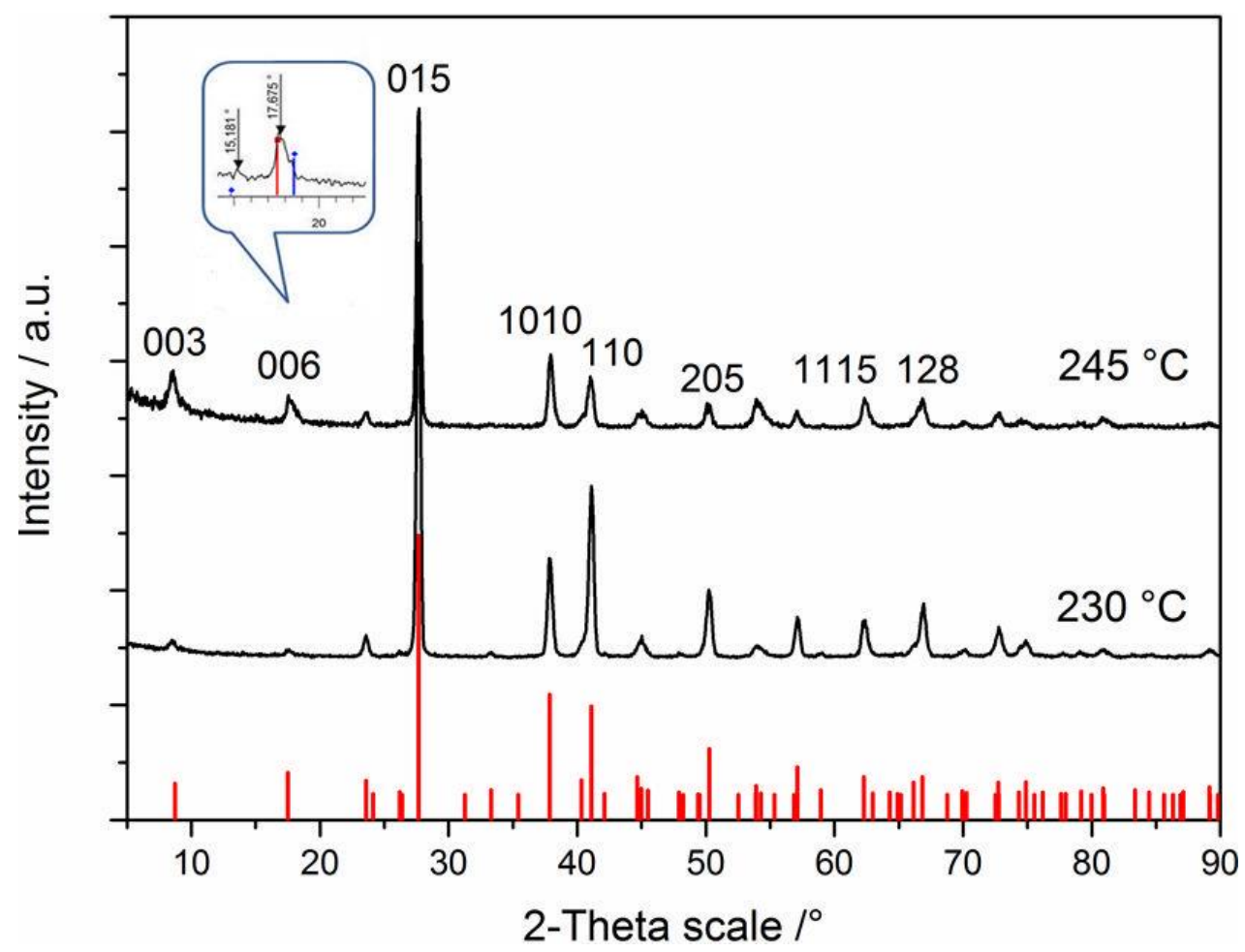

Figure 3. GIXRD pattern of $\mathrm{Bi}_{2} \mathrm{Te}_{3}$ films deposited at $230{ }^{\circ} \mathrm{C}$ and $245^{\circ} \mathrm{C}$ on $\mathrm{Si}(100)$ substrates; most intense peaks are labelled and correspond to the expected reflections of $\mathrm{Bi}_{2} \mathrm{Te}_{3}$ (PDF card 15-0863). In order to gain more information on the film texture and the orientation of the crystallites on the substrate surface, pole figures were measured for two reflections in the $\mathrm{Bi}_{2} \mathrm{Te}_{3}$ film grown at $230{ }^{\circ} \mathrm{C}$ on $\mathrm{Si}(100)$. The (105) pole figure displayed in 2D and 3D representations (Fig. 4) was measured at $20=27.64^{\circ}$. The results showed a strong intensity in the center of the figure at $\psi=90^{\circ}$, and a narrow ring structure at $\psi=45^{\circ}$. The pole figure suggests that the majority of the $\mathrm{Bi}_{2} \mathrm{Te}_{3}$ grains are standing tilted on the substrate surface, as observed in the SEM cross section image (Fig. 2), whereas a minor part of the grains lies flat on the substrate surface. 
A

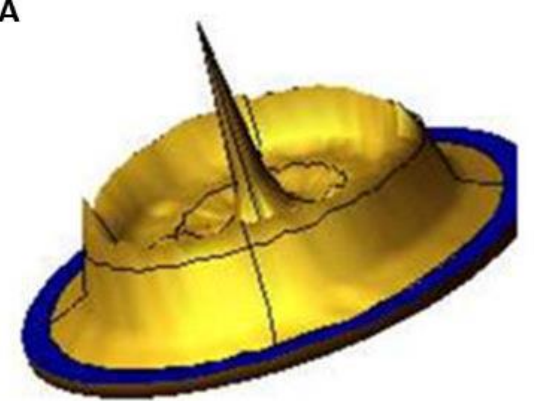

B

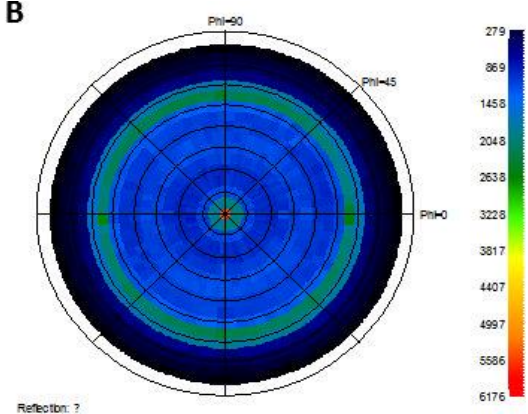

Figure 4. Pole figure for the (105) reflection of the $\mathrm{Bi}_{2} \mathrm{Te}_{3}$ film deposited at $230{ }^{\circ} \mathrm{C}$ on a $\mathrm{Si}(100)$ substrate in 3-dimensional (A) and 2-dimensional (B) representation.

X-Ray photoelectron spectroscopy (XPS) is a surface sensitive method for the determination of the chemical environment of an element. XPS measurements of the $\mathrm{Bi}_{2} \mathrm{Te}_{3}$ film grown at $230^{\circ} \mathrm{C}$ on $\mathrm{Si}(100)$ revealed the expected peaks for $\mathrm{Bi} 4 \mathrm{f} 7 / 2$ and $\mathrm{Bi} 4 \mathrm{f} 5 / 2$ at 157.5 and $162.8 \mathrm{eV}$, as well as the expected peaks for Te $3 d 3 / 2$ and Te $3 d 5 / 2$ at 582.9 and 572.5 eV (Fig. 5), respectively. These values are in good agreement with the reported binding energies of $\mathrm{Bi}_{2} \mathrm{Te}_{3}{ }^{[26-29]}$. In addition, a second set of peaks at higher binding energy is visible for every element, comparable to the binding energies in $\mathrm{Bi}_{2} \mathrm{O}_{3}$ and $\mathrm{TeO}_{2}$. Since only one $\mathrm{O}$ 1s peak at $530.3 \mathrm{eV}$ was observed, the film surface is most likely covered with a thin layer of $\mathrm{Bi}_{2} \mathrm{Te}_{3-\mathrm{x}} \mathrm{O}_{\mathrm{x}}$. Surface oxidation was expected since the film was handled and transferred under ambient conditions, leading to the post-growth oxidation of the material. This was proven by an elemental depth profile analysis, showing a strong decrease of the oxidized species after a short sputtering period. After a sputtering time of $3 \mathrm{~min}$, with an acceleration voltage of $3 \mathrm{kV}$ corresponding to an abrasion of about $10 \mathrm{~nm}$ equivalent to 10 quintuple layers, the intensities of the peaks corresponding to oxygen or carbon are below the detection limit. The surface oxidation of $\mathrm{Bi}_{2} \mathrm{Te}_{3}$ and other tetradymite type materials has been previously reported $^{[30,31]}$.
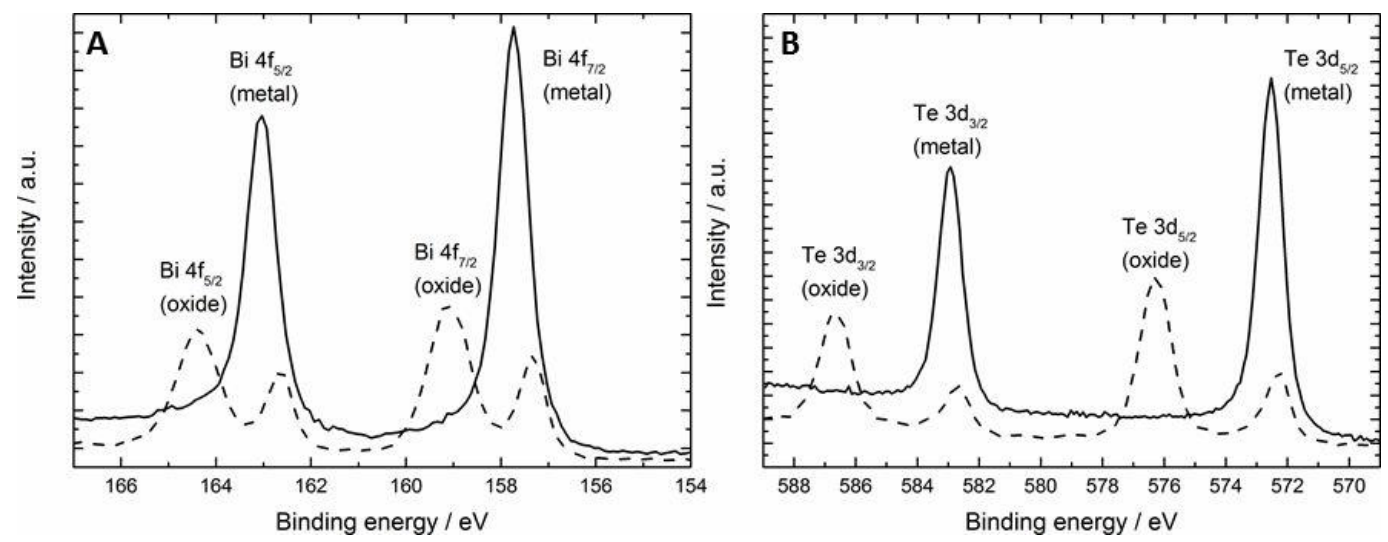

Figure 5. $\mathrm{Bi} 4 \mathrm{f}(\mathrm{A})$ and $\mathrm{Te} 3 \mathrm{~d}(\mathrm{~B})$ core level XPS spectra of $\mathrm{Bi}_{2} \mathrm{Te}_{3}$ thin films before (dashed line) and after sputtering (solid line). 
A transmission electron microscopy (TEM) lamella image was taken by using the focused ion beam technique to inspect the film's microstructure and film-substrate interface in cross-section for the $\mathrm{Bi}_{2} \mathrm{Te}_{3}$ film grown at $230{ }^{\circ} \mathrm{C}$.
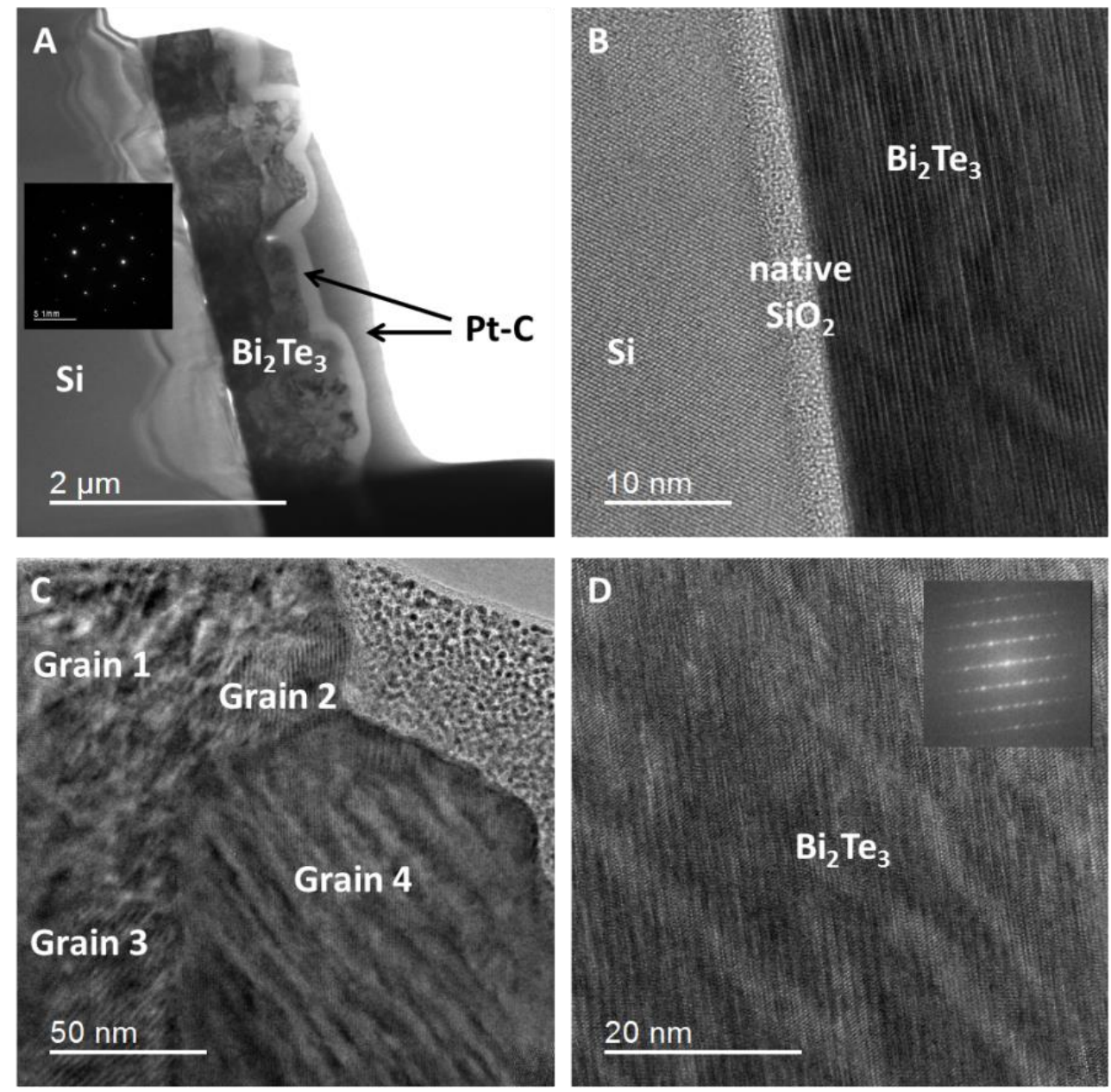

Figure 6. TEM overview image of a FIB cut cross-section lamella (A), HRTEM image of the filmsubstrate interface (B), HRTEM image showing multiple grains close to the film surface (C) and HRTEM image of a region inside a c-oriented $\mathrm{Bi}_{2} \mathrm{Te}_{3}$ grain (D).

An overview of a bright field image of the lamella (Fig. $6 \mathrm{~A}$ ), defocused by $5 \mu \mathrm{m}$ for better visibility of grain boundaries, displays the $\mathrm{Bi}_{2} \mathrm{Te}_{3}$ film with a non-uniform thickness of around 0.8 to $1.0 \mu \mathrm{m}$. In addition, both platinum layers, which were deposited during the FIB process to protect the film, are visible. The selected area electron diffraction (SAED) pattern showing the substrate orientation is presented as inset in Fig. 6A. At a higher magnification, the image reveals a sharp interface between the native $\mathrm{SiO}_{2}$ layer and the $\mathrm{Bi}_{2} \mathrm{Te}_{3}$ film (Fig. 6B). The lattice fringes with a spacing of 1 $\mathrm{nm}$ parallel to the interface are consistent with the initial c-oriented growth of $\mathrm{Bi}_{2} \mathrm{Te}_{3}$ on the $\mathrm{Si}(100)$ substrate, which can be observed at the lower part of the film. Fig. $6 \mathrm{C}$ shows different grains in the upper part of the film. A HRTEM image of a c-oriented grain is shown in Fig. 6D. The inset displays 
the corresponding diffraction pattern, generated by a Fast Fourier Transformation. Furthermore, Figs. 6C and 6D show diagonal stripe patterns that arise from a high density of local stacking faults with a lateral extension of approximately $10 \mathrm{~nm}$. Partial dislocations can terminate such stacking faults.

The low substrate temperatures $\left(215\right.$ to $245^{\circ} \mathrm{C}$ ) in our MOCVD experiments are expected to stimulate the growth of polycrystalline films due to the reduced mobility of the adatoms on the substrate surface during film growth, which is a pre-requisite for the formation of smooth c-oriented films. Comparable findings were observed in the low-temperature MOCVD growth of $\mathrm{Sb}_{2} \mathrm{Te}_{3}$ films using $\left(\mathrm{Et}_{2} \mathrm{Sb}\right)_{2} \mathrm{Te}$ as $\mathrm{SSP}^{[15]}$ and was reported for the ALD growth of $\mathrm{Sb}_{2} \mathrm{Te}_{3}$ and $\mathrm{Bi}_{2} \mathrm{Te}_{3}$ films ${ }^{[32-34]}$, which were also found to be strongly temperature-dependent. The film growth occurred via a Volmer-Weber island growth mechanism and initially formed $\mathrm{Sb}_{2} \mathrm{Te}_{3}$ and $\mathrm{Bi}_{2} \mathrm{Te}_{3}$ grains, which then coalesced to a smooth film with increasing number of cycles, and finally resulted in the formation of three-dimensional polycrystalline $\mathrm{Sb}_{2} \mathrm{Te}_{3}$ and $\mathrm{Bi}_{2} \mathrm{Te}_{3}$ films, respectively. However, we observed the formation of c-oriented platelets in the early stage of the growth process, especially at low temperature (see also fig. 2), which then leads to the formation of a polycrystalline film. One possible reason for this phenomenon could be an influence of the substrate material. It is well known that the morphology of MOCVD- and ALD-grown $\mathrm{Sb}_{2} \mathrm{Te}_{3}$ and $\mathrm{Bi}_{2} \mathrm{Te}_{3}$ films is strongly influenced by the substrate material. For instance, flat c-oriented films were formed on $\mathrm{OH}$-terminated oxide interfaces of $\mathrm{Si}$ or $\mathrm{SiO}_{2}$ substrates ${ }^{[7,32,35,36]}$ as well as $\mathrm{Al}_{2} \mathrm{O}_{3}$ substrates ${ }^{[6]}$. These studies clearly demonstrate that the early state of the film growth shows a strong preference for the growth of coriented platelets, but is always accompanied by the minor formation of platelets, which adopt other orientations. We believe that the $\mathrm{OH}$-termination of the native oxide surface of the $\mathrm{Si}(100)$ substrates, which were used without further chemical or thermal pretreatment, enhances the growth of flat c-oriented platelets in the early stage of the film growth, which is also visible in the SEM images (Fig. 2). The preferential c-oriented growth of $\mathrm{Bi}_{2} \mathrm{Te}_{3}$ on $\mathrm{SiO}_{2}$ at low temperatures is phenomenologically described in Ref. 7. Once the initially formed platelets coalesce into a dense film, the relative growth rates on diverse plane orientations enhance the growth of platelets of different orientations, which results in the formation of rough films with a significant number of grains.

The formation of crystalline, phase-pure $\mathrm{Bi}_{2} \mathrm{Te}_{3}$ films at low substrate temperature is remarkable. $\mathrm{A}$ very recent study on the growth of bismuth telluride thin films by a classical two-precursors MOCVD process using TMBi, DETe and $\mathrm{H}_{2}$ revealed that the quality of the resulting films strongly 
depends on the substrate temperature and the precursor stoichiometry ${ }^{[37]}$. The lowest substrate temperature, at which $\mathrm{Bi}_{2} \mathrm{Te}_{3}$ film growth was observed, was $330^{\circ} \mathrm{C}$. However, phase-pure, crystalline $\mathrm{Bi}_{2} \mathrm{Te}_{3}$ films were obtained at this substrate temperature only in the presence of a very large excess of DETe (Te:Bi ratio > 30), while at higher temperatures, the excess of DETe can be reduced $\left(420^{\circ} \mathrm{C} \text { : Te:Bi } 10 ; 463^{\circ} \mathrm{C} \text { : Te:Bi } 5\right)^{[37]}$. In our case, the use of the SSP Et ${ }_{2} \mathrm{BiTeEt}$ does not require any excess of Te, especially since the precursor decomposes in a stoichiometric reaction on the surface of the substrate, and therefore allows the growth of $\mathrm{Bi}_{2} \mathrm{Te}_{3}$ films at significantly lower temperatures $\left(230^{\circ} \mathrm{C}\right)$. Although surface-controlled reactions are often known to require lower temperatures in comparison to gas phase decomposition reactions, this is not always case. For instance, $t$ $\mathrm{Bu}_{3} \mathrm{Al}$ decomposes in the gas phase at $250{ }^{\circ} \mathrm{C}$, whereas $277^{\circ} \mathrm{C}$ is required on the surface ${ }^{[38]}$. Gas phase reactions are expected to play a major role at high process temperatures, and more importantly, at high process pressures since they are initiated by the collisions between molecules ${ }^{[39]}$. Therefore, low process temperatures $\left(200-250{ }^{\circ} \mathrm{C}\right)$ in combination with reduced pressure conditions (10 mbar) are expected to reduce unwanted gas phase reactions, which could potentially lead to the synthesis of $\mathrm{Bi}_{2} \mathrm{Te}_{3}$ nanoparticle in the gas phase. This whole process could be transformed into a surface-decomposition controlled MOCVD process. In addition, the gas phase decomposition of $\mathrm{Et}_{2} \mathrm{BiTeEt}$ is likely to occur with the breakage of the Bi-Te bond as it's the weakest chemical bond in this compound, thus leading to the formation of $\mathrm{Et}_{2} \mathrm{Bi}$. and EtTe- radical species. EtTe- radicals may either recombine to form $\mathrm{Et}_{2} \mathrm{Te}_{2}$, which is thermally stable as observed with $\mathrm{Me}_{2} \mathrm{Te}_{2}{ }^{[40]}$, or further react to give $\mathrm{Et}_{2} \mathrm{Te}$ and $\mathrm{Te}$ vapor. $\mathrm{Et}_{2} \mathrm{Te}$ is also thermally stable and starts to decompose at $272{ }^{\circ} \mathrm{C}$ according to previous DSC studies ${ }^{[21]}$. In this case, most of the tellurium would be transferred out of the reactor instead of being used in the film growth, and therefore leading to the formation of Bi-rich films. Moreover, the dual source MOCVD growth of $\mathrm{Bi}_{2} \mathrm{Te}_{3}$ films using $\mathrm{Et}_{2} \mathrm{Te}$ and $\mathrm{Et}_{3} \mathrm{Bi}$ only results in $\mathrm{Bi}_{2} \mathrm{Te}_{3}$ film growth at temperatures of at least $350{ }^{\circ} \mathrm{C},{ }^{[41]}$ which is roughly $120^{\circ} \mathrm{C}$ higher than our SSP approach $\mathrm{Et}_{2} \mathrm{BiTeEt}$. These findings are in accordance with previous dual source MOCVD growth studies ${ }^{[4-6]}$. To further exclude the possibility of a gas phase decomposition process, we performed an experiment in which the precursor was passed through a glass tube that was heated up to $215-245{ }^{\circ} \mathrm{C}$ and the substrate was placed in the cold zone at the end of the ov-

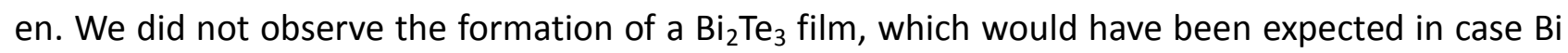
and Te vapor are formed by the gas phase decomposition of the precursor as described above.

The growth of stoichiometric $\mathrm{Bi}_{2} \mathrm{Te}_{3}$ films at $230{ }^{\circ} \mathrm{C}$ strongly supports the surface-controlled decomposition process. In particular, $\mathrm{Et}_{2} \mathrm{BiTeEt}$ does not decompose into $\mathrm{Et}_{2} \mathrm{Bi}$. and $\mathrm{EtTe}$ - radical spe- 
cies in the gas phase, but instead absorbs as intact molecules on the surface of the substrate, thus behaving as a "true" single source precursor. The growth mechanism is likely initiated by the homolytic breakage of the $\mathrm{Bi}$-Te bond, which then triggers the multi-step decomposition of $\mathrm{Et}_{2} \mathrm{BiTeEt}$ on the surface of the substrate. The resulting $\mathrm{Et}_{2} \mathrm{Bi} \cdot$ and $\mathrm{EtTe}$ - radicals undergo disproportionation reactions with subsequent formation of $\mathrm{Et}_{3} \mathrm{Bi}$ and $\mathrm{Et}_{2} \mathrm{Te}$, as well as elemental bismuth and tellurium:

$$
\begin{aligned}
& 6 \mathrm{Et}_{2} \mathrm{BiTeEt} \rightarrow 6 \mathrm{Et}_{2} \mathrm{Bi} \cdot+6 \mathrm{EtTe} \\
& 6 \mathrm{Et}_{2} \mathrm{Bi} \cdot \rightarrow 4 \mathrm{Et}_{3} \mathrm{Bi}+2 \mathrm{Bi} \\
& 6 \mathrm{EtTe} \cdot \rightarrow 3 \mathrm{Et}_{2} \mathrm{Te}+3 \mathrm{Te}
\end{aligned}
$$

These decomposition reactions yield $\mathrm{Bi}$ and $\mathrm{Te}$ in the correct 2:3 stoichiometric ratio, hence results the growth of stoichiometric $\mathrm{Bi}_{2} \mathrm{Te}_{3}$ films. At higher substrate temperatures $\left(245{ }^{\circ} \mathrm{C}\right), \mathrm{Et}_{3} \mathrm{Bi}$ was shown to further decompose (at least partially) and give Bi-rich $\mathrm{Bi}_{4} \mathrm{Te}_{3}$ films. Control of the substrate temperature therefore is crucial for the deposition of phase-pure $\mathrm{Bi}_{2} \mathrm{Te}_{3}$ films, as was also observed in dual source processes ${ }^{[37]}$. In this case, the SSP Et ${ }_{2}$ BiTeEt allows for the use of lower substrate temperatures without any excess of Te precursor. A similar behavior was observed with $\left(\mathrm{Et}_{2} \mathrm{Sb}\right)_{2} \mathrm{Te}$ as a SSP for the MOCVD growth of $\mathrm{Sb}_{2} \mathrm{Te}_{3}$ thin films ${ }^{[15]}$. Despite the Sb:Te ratio of 2:1, its thermal decomposition leads to highly stoichiometric $\mathrm{Sb}_{2} \mathrm{Te}_{3}$ both in solution and in gas phase processes. This is because the formation of volatile $\mathrm{Et}_{3} \mathrm{Sb}$ from the decomposition of $\left(\mathrm{Et}_{2} \mathrm{Sb}\right)_{2} \mathrm{Te}$ easily transfers excess antimony out of the reactor.

\section{Conclusion.}

The potential application of $\mathrm{Et}_{2} \mathrm{BiTeEt}$ as a SSP for the MOCVD growth of $\mathrm{Bi}_{2} \mathrm{Te}_{3}$ films on $\mathrm{Si}(100)$ substrates was demonstrated. The single source approach allows the growth of $\mathrm{Bi}_{2} \mathrm{Te}_{3}$ films at very low substrate temperatures of $230{ }^{\circ} \mathrm{C}$, whereas conventional "dual source" precursors typically require significantly higher substrate temperatures at around $400^{\circ} \mathrm{C}$, as well as large excess of the Te precursor. The low deposition temperature of $230{ }^{\circ} \mathrm{C}$, which is significantly lower than the decomposition temperature of the expected decomposition product $\mathrm{Et}_{2} \mathrm{Te}\left(270{ }^{\circ} \mathrm{C}\right.$ according DSC) and lower than reported MOCVD processes using $\mathrm{Et}_{3} \mathrm{Bi}$ and $\mathrm{Et}_{2} \mathrm{Te}$, indicates that $\mathrm{Et}_{2} \mathrm{BiTeEt}$ is stable in the gas phase under the specified process conditions and adsorbs as intact molecules on the substrate surface, hence serving as single source precursor for the $\mathrm{Bi}_{2} \mathrm{Te}_{3}$ film growth. EDX and XRD studies revealed the formation of highly stoichiometric and phase pure $\mathrm{Bi}_{2} \mathrm{Te}_{3}$ films at 215 and $230{ }^{\circ} \mathrm{C}$, while higher substrate temperatures $\left(245{ }^{\circ} \mathrm{C}\right.$ ) yielded Bi-rich materials with $\mathrm{Bi}_{4} \mathrm{Te}_{3}$ as additional phase. XPS studies proved the existence of a thin oxide layer on the film surface due to 
post-deposition surface oxidation reactions. To the best of our knowledge, this study represents the first report on the successful growth of crystalline $\mathrm{Bi}_{2} \mathrm{Te}_{3}$ thin films at low substrate temperatures using a metal organic SSP. We are currently investigating the low-temperature film growth on different substrates as well as with different SSPs, in which the Bi:Te ratio is systematically varied in order to reveal the influence of both parameters on the morphology (and composition) of the resulting $\mathrm{Bi}_{2} \mathrm{Te}_{3}$ films.

Acknowledgment. F.R. and C.J are grateful to V. Roddatis for support of HRTEM characterization. Financial support by the Deutsche Forschungsgemeinschaft DFG (S. Schulz, project SCHU 1069/201; C. Jooss, project JO 348/12-1) is acknowledged.

\section{References}

[1] C. B. Vining, Nat. Mater. 2009, 8, 83.

[2] D. M.Rowe, CRC Handbook of Thermoelectrics, CRC Press, Boca Raton, 1995.

[3] J. W. G. Bos, H. W. Zandbergen, M. H. Lee, N. P. Ong, R. J. Cava, Phys. Rev. B 2007, 75, 195203.

[4] A. Giani, A. Boulouz, F. Pascal-Delannoy, A. Foucaran, E. Charles, A. Boyer, Mater. Sci. Engin. B 1999, 64, 19.

[5] H. You, S. H. Baek, K.-C. Kim, O. J. Kwon, J.-S. Kim, C. Park, J. Cryst. Growth 2012, 346, 17.

[6] S. Schulz, G. Bendt, J. Sonntag, A. Lorke, U. Hagemann, W. Assenmacher, Semicond. Sci. Technol. 2015, 30, 085021.

[7] S.-W. Kang, K.-M. Jeon, J.-S. Shin, J.-R. Chun, Y.-H. Kim, S. J. Lee, J.-Y. Yun, Chem. Vap. Deposit. $201319,61$.

[8] R. Venkatasubramanian, E. Siivola, T. Colpitts, B. O’Quinn, Nature 2001, 413, 597.

[9] For a recent review article see: T. Dankwort, A.-L. Hansen, M. Winkler, U. Schürmann, J. D. König, D. C. Johnson, N. F. Hinsche, P. Zahn, I. Mertig, W. Bensch, L. Kienle, Phys. Status Solidi A 2016, 213, 662 .

[10] A.-L. Hansen, T. Dankwart, M. Winkler, J. Ditto, D. C. Johnson, J. D. König, K. Bartholomé, L. Kienle, W. Bensch, Chem. Mater. 2014, 26, 6518.

[11] M. Winkler, X. Liu, J. D. König, S. Buller, U. Schürmann, L. Kienle, W. Bensch, H. Böttner, J. Mater. Chem. 2012, 22, 11323.

[12] J. D. König, M. Winkler, S. Buller, W. Bensch, U. Schürmann, L. Kienle, H. Böttner, J. Electronic Mater. 2011, 40, 1266.

[13] S. Heimann, S. Schulz, J. Schaumann, A. Mudring, J. Stötzel, G. Schierning, J. Mater. Chem. C 2015, 3, 10375. 
[14] S. Schulz, S. Heimann, J. Friedrich, M. Engenhorst, G. Schierning, W. Assenmacher, Chem. Mater. 2012, 24, 2228.

[15] G. Bendt, S. Schulz, S. Zastrow, K. Nielsch, Chem. Vap. Deposit. 2013, 19, 235.

[16] S. S. Garje, D. J. Eisler, J. S. Ritch, M. Afzaal, P. O'Brien, T. Chivers, J. Am. Chem. Soc. 2006, 128, 3120.

[17] G. Gupta, J. Kim, Dalton Trans. 2013, 42, 8209.

[18] S. L. Benjamin, C. H. de Groot, A. L. Hector, R. Huang, E. Koukharenko, W. Levason, G. Reid, J. Mater. Chem. C 2015, 3, 423.

[19] R. Huang, S. L. Benjamin, C. Gurnani, Y. Wang, A. L. Hector, W. Levason, G. Reid, C. H. De Groot, Sci. Rep. 2016, 6, 27593.

[20] S. Schulz, Coord. Chem. Rev. 2015, 297-298, 49.

[21] G. Bendt, A. Weber, S. Heimann, W. Assenmacher, O. Prymak, S. Schulz, Dalton Trans. 2015, 44, 14272.

[22] S. L. Benjamin, C. H. de Groot, C. Gurnani, A. L. Hector, R. Huang, W. Levason, G. Reid, J. Mater. Chem. A 2014, 2, 4865.

[23] H. J. Breunig, H. Jawad, J. Organomet. Chem. 1984, 277, 257.

[24] A. J. Asche III, E. G. Ludwig, J. Organomet. Chem. 1986, 308, 289.

[25] S. Heimann, A. Kuczkowski, D. Bläser, C. Wölper, R. Haack, G. Jansen, S. Schulz, Eur. J. Inorg. Chem. 2014, 28, 4858.

[26] C. D. Wagner, A. V. Naumkin, A. Kraut-Vass, J. W. Allison, C. J. Powell, J. R. Jr. Rumble, NIST Standard Reference Database 20, Version 3.4 (web version) (http:/srdata.nist.gov/xps/) 2003.

[27] H. Bando, K. Koizumi, Y. Oikawa, K. Daikohara, V. A. Kulbachinskii, H. Ozaki, J. Phys. Condens. Matter 2000, 12, 5607.

[28] C. R. Thomas, M. K. Vallon, M. G. Frith, H. Sezen, S. K. Kushwaha, R. J. Cava, J. Schwartz, S. L. Bernasek, Chem. Mater. 2016, 28, 35.

[29] E. J. Menke, M. A. Brown, Q. Li, J. C. Hemminger, R. M. Penner, Langmuir 2006, 22, 10564.

[30] M. Loor, G. Bendt, U. Hagemann, C. Wölper, W. Assenmacher, S. Schulz, Dalton Trans. 2016, $45,15326$.

[31] C. R. Thomas, M. K. Vallon, M. G. Frith, H. Sezen, S. K. Kushwaha, R. J. Cava, J. Schwartz, S. L. Bernasek, Chem. Mater. 2016, 28, 35.

[32] T. Sarnet, T. Hatanpa, E. Puukilainen, M. Mattinen, M. Vehkamaki, K. Mizohata, M. Ritala, M. Leskela, J. Phys. Chem. A 2015, 119, 2298.

[33] S. Zastrow, J. Gooth, T. Boehnert, S. Heiderich, W. Toellner, S. Heimann, S. Schulz, K. Nielsch, 


\section{Appendix 1 : Publication 3}

Semicond. Sci. Technol. 2013, 28, 035010.

[34] C. Bae, T. Bohnert, J. Gooth, S. Lim, S. Lee, H. Kim, S. Heimann, S. Schulz, H. Shin, K. Nielsch, Semicond. Sci. Technol. 2014, 29, 064003.

[35] D. Nminibapiel, K. Zhang, M. Tangirala, H. Baumgart, V.S.K. Chakravadhanula, C. Kübel, V. Kochergin, ECS Solid State Sci. Technol. 2014, 3, P95.

[36] M. Rusek, T. Komossa, G. Bendt, S. Schulz, J. Cryst. Growth 2017, 470, 128.

[37] P. I. Kuznetsov, V. O. Yapaskurt, B. S. Shchamkhalova, V. D. Shcherbakov, G. G. Yakushcheva,. A. Luzanov, V. A. Jitov, J. Cryst. Growth 2016, 455, 122.

[38] A. C. Jones, P. O'Brien, CVD of Compounds Semiconductors: Precursor Synthesis, Development and Applications, p. 152 ff, VCH, Weinheim, 1997.

[39] J.-H. Park, T. S. Sudarshan, Chemical Vapor Deposition, p. 16 ff, ASM International, 2001.

[40] a) J. E. Hails, D. J. Cole-Hamilton, W. Bell, J. Cryst. Growth 1994, 145, 596-601; b) J. E. Hails, D. J. Cole-Hamilton, A. E. D. McQueen, J. Cryst. Growth 1998, 183, 594-603.

[41] G. Bendt, S. Schulz, unpublished results. 



\section{Appendix 2: Structural and thermoelectrical character- ization of epitaxial $\mathrm{Sb}_{2} \mathrm{Te}_{3}$ high quality thin films grown by thermal evaporation}

This chapter consists of the accepted manuscript of the original article

Structural and thermoelectrical characterization of epitaxial $\mathrm{Sb}_{2} \mathrm{Te}_{3}$ high quality thin films grown by thermal evaporation

G. Bendt ${ }^{1}$, K. Kaiser ${ }^{1}$, A. Heckel ${ }^{1}$, F. Rieger ${ }^{2}$, D. Oing ${ }^{3}$, A. Lorke ${ }^{3}$, N. Perez Rodriguez ${ }^{4}$, G. Schierning ${ }^{4}$, C. Jooss ${ }^{2}$, and S. Schulz ${ }^{1}$

${ }^{1}$ Institute of Inorganic Chemistry and Center for Nanointegration Duisburg-Essen (Cenide), University of Duisburg - Essen, 45141 Essen, Germany

${ }^{2}$ Institute of Material Physics, University of Göttingen, 37077 Göttingen, Germany

${ }^{3}$ Faculty of Physics and Center for Nanointegration Duisburg-Essen (CENIDE), University of Duisburg-Essen, Lotharstraße 1, 47048 Duisburg, Germany

${ }^{4}$ Institute for Metallic Materials, Leibniz Institute for Solid State and Materials Research Dresden (IFW Dresden), Helmholtzstr. 20, 01069 Dresden, Germany

Originally published in Semiconductor Science and Technology 33:105002, 2018. [110] DOI: $10.1088 / 1361-6641 /$ aad7a3

After the embargo time, a CC BY-NC-ND 3.0 license applies to the accepted manuscript. This thesis has been published within the embargo time of ref. [110]. For that reason the manuscript is not included in the present version of the thesis. 



\section{References}

[1] W. Haken. 3. Beitrag zur Kenntnis der thermoelektrischen Eigenschaften der Metallegierungen. Ann. Phys., 337:291-336, 1910.

[2] M. Telkes. The Efficiency of Thermoelectric Generators. I. J. Appl. Phys., 18:1116$1127,1947$.

[3] H. J. Goldsmid and R. W. Douglas. The use of semiconductors in thermoelectric refrigeration. Br. J. Appl. Phys., 5:386-390, 1954.

[4] T. C. Harman, B. Paris, S. E. Miller, and H. L. Goering. Preparation and some physical properties of $\mathrm{Bi}_{2} \mathrm{Te}_{3}, \mathrm{Sb}_{2} \mathrm{Te}_{3}$, and $\mathrm{As}_{2} \mathrm{Te}_{3}$. J. Phys. Chem. Solids, 2:181190, 1957.

[5] U. Birkholz. Untersuchung der intermetallischen Verbindung $\mathrm{Bi}_{2} \mathrm{Te}_{3}$ sowie der festen Lösungen $\mathrm{Bi}_{2-\mathrm{x}} \mathrm{Sb}_{\mathrm{x}} \mathrm{Te}_{3}$ und $\mathrm{Bi}_{2} \mathrm{Te}_{3-\mathrm{x}} \mathrm{Se}_{\mathrm{x}}$ hinsichtlich ihrer Eignung als Material für Halbleiter-Thermoelemente. Z. Naturforschg., 13a:780-792, 1958.

[6] K. S̆mirous and L. S̆tourač. Notizen:Feste Lösungen von $\mathrm{Bi}_{2} \mathrm{Te}_{3}$ und $\mathrm{Sb}_{2} \mathrm{Te}_{3}$ als p-leitende Werkstoffe für Halbleiterthermoelemente. Z. Naturforsch., 14a:848-849, 1959 .

[7] R. Venkatasubramanian, E. Siivola, T. Colpitts, and B. O'Quinn. Thin-Film Thermoelectric Devices With High Room-Temperature Figures of Merit. Nature, 413:597-602, 2001.

[8] M. Winkler, X. Liu, U. Schürmann, J. D. König, L. Kienle, W. Bensch, and H. Böttner. Current Status in Fabrication, Structural and Transport Property Characterization, and Theoretical Understanding of $\mathrm{Bi}_{2} \mathrm{Te}_{3} / \mathrm{Sb}_{2} \mathrm{Te}_{3}$ Superlattice Systems. $Z$. Anorg. Allg. Chem., 638:2441-2454, 2012.

[9] T. Dankwort, A.-L. Hansen, M. Winkler, U. Schürmann, J. D. Koenig, D. C. Johnson, N. F. Hinsche, P. Zahn, I. Mertig, W. Bensch, and L. Kienle. Nanostructure, thermoelectric properties, and transport theory of $\mathrm{V}_{2} \mathrm{VI}_{3}$ and $\mathrm{V}_{2} \mathrm{VI}_{3} / \mathrm{IV}-\mathrm{VI}$ based superlattices and nanomaterials. Phys. Status Solidi A, 213:662-671, 2016. 
[10] T. J. Seebeck. Magnetische Polarisation der Metalle und Erze durch TemperaturDifferenz. Abhandlungen der Königlichen Akademie der Wissenschaften zu Berlin, 7:265-373, 1822.

[11] J. C. A. Peltier. Nouvelles expériences sur la caloricité des courants électrique. Ann. Chim. Phys., 56:371-386, 1834.

[12] D. Bhatia, S. Bairagi, S. Goel, and M. Jangra. Pacemakers charging using body energy. J. Pharm. Bioallied Sci., 2:51-54, 2010.

[13] E. Altenkirch. Über den Nutzeffekt der Thermosäule. Phys. Z., 10:560-568, 1909.

[14] E. Altenkirch. Elektrothermische Kälteerzeugung und reversible elektrische Heizung. Phys. Z., 12:920-924, 1911.

[15] T. H. Geballe and G. W. Hull. Seebeck Effect in Germanium. Phys. Rev., 94:1134$1140,1954$.

[16] T. H. Geballe and G. W. Hull. Seebeck Effect in Silicon. Phys. Rev., 98:940-947, 1955.

[17] A. F. Joffe. Semiconductor, Thermoelements and Thermoelectric Cooling. Infosearch Ltd., London, 1957.

[18] A. F. Joffe and L. S. Stil'bans. Physical problems of thermoelectricity. Rep. Prog. Phys., 22:167-203, 1959.

[19] G. D. Mahan. Introduction to thermoelectrics. APL Materials, 4:104806, 2016.

[20] A. Volta. Nuova memoria sull'elettricità animale del Sig. Don Alessandro Volta ... in alcune lettere al Sig. Ab. Anton Maria Vassalli ... Annali di chimica e storia naturale, 5:132-144, 1794 .

[21] A. K. Pattanaik and A. Srinivasan. Electrical and optical studies on Pb-modified amorphous Ge-Se-Te films. Semicond. Sci. Technol., 19:157-161, 2004.

[22] G. F. Snyder and E. S. Toberer. Complex thermoelectric materials. Nat. Mater., $7: 105-114,2008$.

[23] S. I. Kim, K. H. Lee, H. A Mun, H. S. Kim, S. W. Hwang, J. W. Roh, D. J. Yang, W. H. Shin, X. S. Li, Y. H. Lee, G. J. Snyder, and S. W. Kim. Dense dislocation arrays embedded in grain boundaries for high-performance bulk thermoelectrics. Science, 348:109-114, 2015.

[24] L.-D. Zhao, S.-H. Lo, Y. Zhang, H. Sun, G. Tan, C. Uher, C. Wolverton, V. P. Dravid, and M. G. Kanatzidis. Ultralow thermal conductivity and high thermoelectric figure of merit in SnSe crystals. Nature, 508:373-377, 2014. 
[25] C. Chiritescu, D. G. Cahill, N. Nguyen, D. Johnson, A. Bodapati, P. Keblinski, and P. Zschack. Ultralow Thermal Conductivity in Disordered, Layered $\mathrm{WSe}_{2}$ Crystals. Science, 315:351-353, 2007.

[26] H. Ohta, K. Sugiura, and K. Koumoto. Recent Progress in Oxide Thermoelectric Materials: p-Type $\mathrm{Ca}_{3} \mathrm{Co}_{4} \mathrm{O}_{9}$ and n-Type $\mathrm{SrTiO}_{3}{ }^{-}$. Inorg. Chem., 47:8429-8436, 2008 .

[27] L. Wu, Q. Meng, C. Jooss, J.-C. Zheng, H. Inada, D. Su, Q. Li, and Y. Zhu. Origin of Phonon Glass-Electron Crystal Behavior in Thermoelectric Layered Cobaltate. Adv. Funct. Mater., 23:5728-5736, 2013.

[28] H. C. Wang, C. L. Wang, W. B. Su, J. Liu, H. Peng, Y. Sun, J. L. Zhang, M. L. Zhao, J. C. Li, N. Yin, and L. M. Mei. Synthesis and thermoelectric performance of Ta doped $\mathrm{Sr}_{0.9} \mathrm{La}_{0.1} \mathrm{TiO}_{3}$ ceramics. Ceram. Int., 37:2609-2613, 2011.

[29] R. Basu, S. Bhattacharya, R. Bhatt, M. Roy, S. Ahmad, A. Singh, M. Navaneethan, Y. Hayakawa, D. K. Aswal, and S. K. Gupta. Improved thermoelectric performance of hot pressed nanostructured n-type SiGe bulk alloys. J. Mater. Chem. A, 2:69226930, 2014.

[30] S. N. Girard, X. Chen, F. Meng, A. Pokhrel, J. Zhou, L. Shi, and S. Jin. Thermoelectric Properties of Undoped High Purity Higher Manganese Silicides Grown by Chemical Vapor Transport. Chem. Mater., 26:5097-5104, 2014.

[31] S. M. Kauzlarich, S. R. Brown, and G. J. Snyder. Zintl phases for thermoelectric devices. Dalton Trans., 0:2099-2107, 2007.

[32] F. Heusler. Über magnetische Manganlegierungen. Verh. Dtsch. Phys. Ges., 5:219, 1903.

[33] K. Manna, Y. Sun, L. Muechler, J. Kübler, and C. Felser. Heusler, Weyl and Berry. Nat. Rev. Mat., 3:244-256, 2018.

[34] W. Lu, W. Zhang, and L. Chen. Thermoelectric properties of $\left(\mathrm{Fe}_{1-\mathrm{x}} \mathrm{Co}_{\mathrm{x}}\right)_{2} \mathrm{VAl}$ Heusler-type compounds. J. Alloys Compd., 484:812-815, 2009.

[35] M. Gürth, G. Rogl, V. V. Romaka, A. Grytsiv, E. Bauer, and P. Rogl. Thermoelectric high ZT half-Heusler alloys $\mathrm{Ti}_{1-\mathrm{x}-\mathrm{y}} \mathrm{Zr}_{\mathrm{x}} \mathrm{Hf}_{\mathrm{y}} \mathrm{NiSn}(0 \leq x \leq 1 ; 0 \leq y \leq 1)$. Acta Mater., 104:210-222, 2016.

[36] G. S. Nolas, D. T. Morelli, and T. M. Tritt. Skutterudites: A Phonon-Glass-Electron Crystal Approach to Advanced Thermoelectric Energy Conversion Applications. Annu. Rev. Mater. Sci., 29:89-116, 1999.

[37] T. Takabatake, K. Suekuni, T. Nakayama, and E. Kaneshita. Phonon-glass electroncrystal thermoelectric clathrates: Experiments and theory. Rev. Mod. Phys., 86:669716, 2014. 
[38] M. Rull-Bravo, A. Moure, J. F. Fernández, and M. Martín-González. Skutterudites as thermoelectric materials: revisited. RSC Adv., 5:41653-41667, 2015.

[39] X. Shi, J. Yang, J. R. Salvador, M. Chi, J. Y. Cho, H. Wang, S. Bai, J. Yang, W. Zhang, and L. Chen. Multiple-Filled Skutterudites: High Thermoelectric Figure of Merit through Separately Optimizing Electrical and Thermal Transports. J. Am. Chem. Soc., 133:7837-7846, 2011.

[40] A. Saramat, G. Svensson, A. E. C. Palmqvist, C. Stiewe, E. Mueller, D. Platzek, S. G. K. Williams, D. M. Rowe, J. D. Bryan, and G. D. Stucky. Large thermoelectric figure of merit at high temperature in Czochralski-grown clathrate $\mathrm{Ba}_{8} \mathrm{Ga}_{16} \mathrm{Ge}_{30} . J$. Appl. Phys., 99:023708, 2006.

[41] J. I. Frenkel. Wave Mechanics. Elementary Theory. Clarendon Press, Oxford, 1932.

[42] C. Kittel. Einführung in die Festkörperphysik. 14., überarbeitete und erweiterte Auflage. Oldenbourg Wissenschaftsverlag, 2005.

[43] D. Zheng, S. Tanaka, K. Miyazaki, and M. Takashiri. Evaluation of Specific Heat, Sound Velocity and Lattice Thermal Conductivity of Strained Nanocrystalline Bismuth Antimony Telluride Thin Films. J. Electron. Mater., 44:1679-1687, 2015.

[44] H. B. G. Casimir. Note on the conduction of heat in crystals. Physica, 5:495-500, 1938.

[45] J. W. Strutt. XV. On the light from the sky, its polarization and colour. The London, Edinburgh and Dublin Philosophical Magazine and Journal of Science, 41:107-120, 1871.

[46] P. G. Klemens. The Scattering of Low-Frequency Lattice Waves by Static Imperfections. Proc. Phys. Soc. A, 68:1113-1128, 1955.

[47] R. Peierls. Zur kinetischen Theorie der Wärmeleitung in Kristallen. Annalen der Physik, 395:1055-1101, 1929.

[48] J. Callaway. Model for Lattice Thermal Conductivity at Low Temperatures. Phys. Rev., 113:1046-1051, 1959.

[49] P. G. Klemens. Thermal Conductivity and Lattice Vibrational Modes. Solid State Physics, 7:1-98, 1958.

[50] M. G. Holland. Analysis of Lattice Thermal Conductivity. Phys. Rev., 132:24612471, 1963.

[51] Y.-J. Han. Intrinsic thermal-resistive process of crystals: Umklapp processes at low and high temperatures. Phys. Rev. B, 54:8977-8980, 1996.

[52] T. Feng, L. Lindsay, and X. Ruan. Four-phonon scattering significantly reduces intrinsic thermal conductivity of solids. Phys. Rev. B, 96:161201(R), 2017. 
[53] D. Das, S. Das, P. Singha, K. Malik, A. K. Deb, A. Bhattacharyya, V. A. Kulbachinskii, R. Basu, S. Dhara, S. Bandyopadhyay, and A. Banerjee. Evolution of phonon anharmonicity in Se-doped $\mathrm{Sb}_{2} \mathrm{Te}_{3}$ thermoelectrics. Phys. Rev. B, 96:064116, 2017.

[54] D. G. Cahill, S. K. Watson, and R. O. Pohl. Lower Limit to the Thermal Conductivity of Disordered Crystals. Phys. Rev. B, 46:6131-6140, 1992.

[55] J. R. Drabble and C. H. L. Goodman. Chemical bonding in bismuth telluride. J. Phys. Chem. Solids, 5:142-144, 1958.

[56] J. Horák, K. Čermák, and L. Koudelka. Energy formation of antisite defects in doped $\mathrm{Sb}_{2} \mathrm{Te}_{3}$ and $\mathrm{Bi}_{2} \mathrm{Te}_{3}$ crystals. J. Phys. Chem. Solids, 47:805-809, 1986.

[57] W. M. Haynes, editor. CRC Handbook of Chemistry and Physics. CRC Press, Boca Raton, FL, 97th edition, 2016.

[58] W.-S. Kim. Solid state phase equilibria in the Pt-Sb-Te system. J. Alloys Compd., 252:166-171, 1997.

[59] Y. Feutelais, B. Legendre, N. Rodier, and V. Agafonov. A study of the phases in the bismuth - tellurium system. Mater. Res. Bull., 28:591-596, 1993.

[60] I. Lefebvre, M. Lannoo, G. Allan, A. Ibanez, J. Fourcade, J. C. Jumas, and E. Beaurepaire. Electronic Properties of Antimony Chalcogenides. Phys. Rev. Lett., 59:2471-2474, 1987.

[61] Y. L. Chen, J. G. Analytis, J.-H. Chu, Z. K. Liu, S.-K. Mo, X. L. Qi, H. J. Zhang, D. H. Lu, X. Dai, Z. Fang, S. C. Zhang, I. R. Fisher, Z. Hussain, and Z.-X. Shen. Experimental Realization of a Three-Dimensional Topological Insulator, $\mathrm{Bi}_{2} \mathrm{Te}_{3}$. Science, 325:178-181, 2009.

[62] M. Stordeuer and W. Heiliger. Anisotropy of the Thermopower of $\mathrm{p}-\mathrm{Sb}_{2} \mathrm{Te}_{3}$. Phys. Status Solidi B, 78:K103-K106, 1976.

[63] H.-W. Jeon, H.-P. Ha, D.-B. Hyun, and J.-D. Shim. Electrical and Thermoelectric Properties of Undoped $\mathrm{Bi}_{2} \mathrm{Te}_{3}-\mathrm{Sb}_{2} \mathrm{Te}_{3}$ and $\mathrm{Bi}_{2} \mathrm{Te}_{3}-\mathrm{Sb}_{2} \mathrm{Te}_{3}-\mathrm{Sb}_{2} \mathrm{Se}_{3}$ Single Crystals. $J$. Phys. Chem. Solids, 52:579-585, 1991.

[64] J. Nagao, M. Ferhat, E. Hatta, and K. Mukasa. Anisotropic Factor of Electrical Conductivity in p-Bi $\mathrm{Te}_{3}$ Crystals. Phys. Stat. Sol. B, 219:347-349, 2000.

[65] P Lošták, Č. Drašar, A. Krejčová, L. Beneš, J.S. Dyck, W. Chen, and C. Uher. Preparation and some physical properties of tetradymite-type $\mathrm{Sb}_{2} \mathrm{Te}_{3}$ single crystals doped with CdS. J. Cryst. Growth, 222:565-573, 2001.

[66] J. P. Fleurial, L. Gailliard, R. Triboulet, H. Scherrer, and S. Scherrer. Thermal properties of high quality single crystals of bismuth telluride-Part I: Experimental characterization. J. Phys. Chem. Solids, 49:1237-1247, 1988. 
[67] J. M. Yáñez-Limón, J. González-Hernández, J. J. Alvaro-Gil, I. Delgadillo, and H. Vargas. Thermal and electrical properties of the Ge:Sb:Te system by photoacoustic and Hall measurements. Phys. Rev. B, 52:16 321-16 324, 1995.

[68] C. V. Manzano, B. Abad, M. Muñoz Rojo, Y. R. Koh, S. L. Hodson, A. M. Lopez Martinez, X. Xu, A. Shakouri, T. D. Sands, T. Borca-Tasciuc, and M. MartinGonzalez. Anisotropic Effects on the Thermoelectric Properties of Highly Oriented Electrodeposited $\mathrm{Bi}_{2} \mathrm{Te}_{3}$ Films. Scientific Reports, 6:19129, 2016.

[69] X. X. Yang, Z. F. Zhou, Y. Wang, R. Jiang, and W. T. Zheng et al. Raman spectroscopy determination of the Debye temperature and atomic cohesive energy of CdS, CdSe, $\mathrm{Bi}_{2} \mathrm{Se}_{3}$, and $\mathrm{Sb}_{2} \mathrm{Te}_{3}$ nanostructures. J. Appl. Phys., 112:083508, 2012.

[70] J. Q. Jenkins, J. A. Rayne, and R. W. Ure Jr. Elastic Moduli and Phonon Properties of $\mathrm{Bi}_{2} \mathrm{Te}_{3}$. Phys. Rev. B, 5:3171-3184, 1972.

[71] M. König, S. Wiedmann, Ch. Brüne, A. Roth, H. Buhmann, L. W. Molenkamp, X.-L. Qi, and S.-C. Zhang. Quantum Spin Hall Insulator State in HgTe Quantum Wells. Science, 318:766-770, 2007.

[72] J. Liang, L. Cheng, J. Zhang, H. Liu, and Z. Zhang. Maximizing the thermoelectric performance of topological insulator $\mathrm{Bi}_{2} \mathrm{Te}_{3}$ films in the few-quintuple layer regime. Nanoscale, 8:8855-8862, 2016.

[73] D. Hsieh, Y. Xia, D. Qian, L. Wray, F. Meier, J. H. Dil, J. Osterwalder, L. Patthey, A. V. Fedorov, H. Lin, A. Bansil, D. Grauer, Y. S. Hor, R. J. Cava, and M. Z. Hasan. Observation of Time-Reversal-Protected Single-Dirac-Cone TopologicalInsulator States in $\mathrm{Bi}_{2} \mathrm{Te}_{3}$ and $\mathrm{Sb}_{2} \mathrm{Te}_{3}$. Phys. Rev. Lett., 103:146401, 2009.

[74] H. B. Zhang, H. L. Yu, and G. W. Yang. Experimental evidence of the nanoscaled topological metallic surface state of $\mathrm{Bi}_{2} \mathrm{Te}_{3}$ and $\mathrm{Sb}_{2} \mathrm{Te}_{3}$ films. EPL, 95:56002, 2011.

[75] N. Xu, Y. Xu, and J. Zhu. Topological insulators for thermoelectrics. npj Quantum Materials, 2:51, 2017.

[76] G. L. Sun, L. L. Li, X. Y. Qin, D. Li, T. H. Zou, H. X. Xin, B. J. Ren, J. Zhang, Y. Y. Li, and X. J. Li. Enhanced thermoelectric performance of nanostructured topological insulator $\mathrm{Bi}_{2} \mathrm{Se}_{3}$. Appl. Phys. Lett., 106:053102, 2015.

[77] N.-W. Park, W.-Y. Lee, J.-E. Hong, T.-H. Park, S.-G. Yoon, H. Im, H. S. Kim, and S.-K. Lee. Effect of grain size on thermal transport in post-annealed antimony telluride thin films. Nanoscale Research Letters, 10:20, 2015.

[78] M. Winkler, X. Liu, A.-L. Hansen, J. D. König, W. Bensch, L. Kienle, H. Böttner, and K. Bartholomé. Sputtered n-type $\mathrm{Bi}_{2} \mathrm{Te}_{3} /(\mathrm{Bi}, \mathrm{Sb})_{2} \mathrm{Te}_{3}$ superlattice systems. Nanothermoelectrics, 1:1-9, 2013.

[79] Ctirad Uher, editor. Material Aspects of Thermoelectricity. CRC Press, 2016. 
[80] B.-T. Wang, P. Souvatzis, O. Eriksson, and P. Zhang. Lattice dynamics and chemical bonding in $\mathrm{Sb}_{2} \mathrm{Te}_{3}$ from first-principles calculations. J. Chem. Phys., 142:174702, 2015 .

[81] D. Bessas, I. Sergueev, H.-C. Wille, J. Perßon, D. Ebling, and R. P. Hermann. Lattice dynamics in $\mathrm{Bi}_{2} \mathrm{Te}_{3}$ and $\mathrm{Sb}_{2} \mathrm{Te}_{3}$ : Te and $\mathrm{Sb}$ density of phonon states. Phys. Rev. B, 86:224301, 2012.

[82] O. Hellman and D. A. Broido. Phonon thermal transport in $\mathrm{Bi}_{2} \mathrm{Te}_{3}$ from first principle. Phys. Rev. B, 90:134309, 2014.

[83] Y. Wang, J. J. Wang, W. Y. Wang, Z. G. Mei, S. L. Shang, L. Q. Chen, and Z. K. Liu. A mixed-space approach to first-principles calculations of phonon frequencies for polar materials. J. Phys.: Condens. Matter, 22:202201, 2010.

[84] F. Rieger, K. Kaiser, G. Bendt, V. Roddatis, P. Thiessen, S. Schulz, and C. Jooss. Low intrinsic c-axis thermal conductivity in PVD grown epitaxial $\mathrm{Sb}_{2} \mathrm{Te}_{3}$ films. $J$. Appl. Phys., 123:175108, 2018.

[85] M. de Jong, W. Chen, T. Angsten, A. Jain, R. Notestine, A. Gamst, M. Sluiter, C. Krishna Ande, S. van der Zwaag, J. J. Plata, C. Toher, S. Curtarolo, G. Ceder, K. A. Persson, and M. Asta. Charting the complete elastic properties of inorganic crystalline compounds. Sci. Data, 2:150009, 2015.

[86] K. H. Park, M. Mohamed, Z. Aksamija, and U. Ravaioli. Phonon scattering due to van der Waals forces in the lattice thermal conductivity of $\mathrm{Bi}_{2} \mathrm{Te}_{3}$ thin films. $J$. Appl. Phys., 117:015103, 2015.

[87] C. K. Gan and C. H. Lee. Anharmonic phonon effects on linear thermal expansion of trigonal bismuth selenide and antimony telluride crystals. Comput. Mater. Sci., 151:49-52, 2018.

[88] Y. Tian, S. Jia, R. J. Cava, R. Zhong, J. Schneeloch, G. Gu, and K. S. Burch. Understanding the evolution of anomalous anharmonicity in $\mathrm{Bi}_{2} \mathrm{Te}_{3-\mathrm{x}} \mathrm{Se}_{\mathrm{x}}$. Phys. Rev. B, 95:094104, 2017.

[89] P. Erhart, P. Hyldgaard, and D. O. Lindroth. Microscopic Origin of Thermal Conductivity Reduction in Disordered van der Waals Solids. Chem. Mater., 27:5511$5518,2015$.

[90] S. Lee, K. Esfarjani, T. Luo, J. Zhou, Z. Tian, and G. Chen. Resonant bonding leads to low lattice thermal conductivity. Nat. Commun., 5:3525, 2014.

[91] P. L. Kapitza. Heat Transfer and Superfluidity of Helium II. Phys. Rev., 60:354-355, 1941.

[92] W. H. Keesom and A. P. Keesom. On the heat conductivity of liquid helium. Physica, 3:359-360, 1936. 
[93] N. Kürti, B. V. Rollin, and F. Simon. Preliminary experiments on temperature equilibria at very low temperatures. Physica, 3:266-274, 1936.

[94] E. T. Swartz and R. O. Pohl. Thermal boundary resistance. Rev. Mod. Phys., 61:605-668, 1989.

[95] R. Viswanathan. Heat capacity of sapphire between 2 and $10 \mathrm{~K}$ by ac technique. J. Appl. Phys., 46:4086-4087, 1975.

[96] R. M. Costescu, M. A. Wall, and D. G. Cahill. Thermal conductance of epitaxial interfaces. Phys. Rev. B, 67:054302, 2003.

[97] B. C. Gundrum, D. G. Cahill, and R. S. Averback. Thermal conductance of metalmetal interfaces. Phys. Rev. B, 72:245426, 2005.

[98] H.-K. Lyeo and D. G. Cahill. Thermal conductance of interfaces between highly dissimilar materials. Phys. Rev. B, 73:144301, 2006.

[99] S. Zastrow, J. Gooth, T. Boehnert, S. Heiderich, W. Toellner, S. Heimann, S. Schulz, and K. Nielsch. Thermoelectric transport and Hall measurements of low defect $\mathrm{Sb}_{2} \mathrm{Te}_{3}$ thin films grown by atomic layer deposition. Semicond. Sci. Technol., 28:035010, 2013.

[100] T. Sarnet, T. Hatanpaää, E. Puukilainen, M. Mattinen, M. Vehkamäki, K. Mizohata, M. Ritala, and M. Leskel"a. Atomic Layer Deposition and Characterization of $\mathrm{Bi}_{2} \mathrm{Te}_{3}$ Thin Films. J. Phys. Chem. A, 119:2298-2306, 2015.

[101] X. Zhang, Z. Zeng, C. Shen, Z. Zhang, Z. Wang, C. Lin, and Z. Hu. Investigation on the electrical transport properties of highly (001)-textured $\mathrm{Sb}_{2} \mathrm{Te}_{3}$ films deposited by molecular beam epitaxy. J. Appl. Phys., 115:024307, 2014.

[102] J. Krumrain, G. Mussler, S. Borisova, T. Stoica, L. Plucinski, C. M. Schneider, and D. Grützmacher. MBE growth optimization of topological insulator $\mathrm{Bi}_{2} \mathrm{Te}_{3}$ films. J. Cryst. Growth, 324:115-118, 2011.

[103] P. Fan, Z.-H. Zheng, G.-X Liang, X.-M. Cai, and D.-P. Zhang. CompositionDependent Characterization of $\mathrm{Sb}_{2} \mathrm{Te}_{3}$ Thin Films Prepared by Ion Beam Sputtering Deposition. Chin. Phys. Lett., 27:087201, 2010.

[104] Z. H. Zheng, P. Fan, T. B. Chen, Z. K. Cai, P. J. Liu, G. X. Liang, D. P. Zhang, and X. M. Cai. Optimization in fabricating bismuth telluride thin films by ion beam sputtering deposition. Thin Solid Films, 520:5245-5248, 2012.

[105] Y. Saito, P. Fons, A. V. Kolobov, and J. Tominaga. Self-organized van der Waals epitaxy of layered chalcogenide structures. Phys. Status Solidi B, 252:2151-2158, 2015. 
[106] X. Wang, H. He, N. Wang, and L. Miao. Effects of annealing temperature on thermoelectric properties of $\mathrm{Bi}_{2} \mathrm{Te}_{3}$ films prepared by co-sputtering. Appl. Surf. Sci., 276:539-542, 2013.

[107] I. Hilmi, A. Lotnyk, J. W. Gerlach, P. Schumacher, and B. Rauschenbach. Research Update: Van-der-Waals epitaxy of layered chalcogenide $\mathrm{Sb}_{2} \mathrm{Te}_{3}$ thin films grown by pulsed laser deposition. APL Materials, 5:050701, 2017.

[108] A. Dauscher, A. Thomy, and H. Scherrer. Pulsed laser deposition of $\mathrm{Bi}_{2} \mathrm{Te}_{3}$ thin films. Thin Solid Films, 280:61-66, 1996.

[109] L. M. Goncalves, P. Alpuim, A. G. Rolo, and J. H. Correia. Thermal co-evaporation of $\mathrm{Sb}_{2} \mathrm{Te}_{3}$ thin-films optimized for thermoelectric applications. Thin Solid Films, 519:4152-4157, 2011.

[110] G. Bendt, K. Kaiser, A. Heckel, F. Rieger, D. Oing, A. Lorke, N. Perez Rodriguez, G. Schierning, C. Jooss, and S. Schulz. Structural and thermoelectrical characterization of epitaxial $\mathrm{Sb}_{2} \mathrm{Te}_{3}$ high quality thin films grown by thermal evaporation. Semicond. Sci. Technol., 33:105002, 2018.

[111] L. M. Goncalves, C. Couto, P. Alpuim, A. G. Rolo, F. Völklein, and J. H. Correia. Optimization of thermoelectric properties on $\mathrm{Bi}_{2} \mathrm{Te}_{3}$ thin films deposited by thermal co-evaporation. Thin Solid Films, 518:2816-2821, 2010.

[112] G. Bendt, S. Zastrow, K. Nielsch, P. S. Mandal, J. Sánchez-Barriga, O. Rader, and S. Schulz. Deposition of topological insulator $\mathrm{Sb}_{2} \mathrm{Te}_{3}$ films by an MOCVD process. J Mater. Chem. A, 2:8215-8222, 2014.

[113] H. You, S. H. Baek, K.-C. Kim, O-J. Kwon, J.-S. Kim, and C. Park. Growth and thermoelectric properties of $\mathrm{Bi}_{2} \mathrm{Te}_{3}$ films deposited by modified MOCVD. J. Cryst. Growth, 346:17-21, 2012.

[114] C. Zhang, Z. Peng, Z. Li, L. Yu, K. A. Khor, and Q. Xiong. Controlled growth of bismuth antimony telluride $\mathrm{Bi}_{\mathrm{x}} \mathrm{Sb}_{2-\mathrm{x}} \mathrm{Te}_{3}$ nanoplatelets and their bulk thermoelectric nanocomposites. Nano Energy, 15:688-696, 2015.

[115] F. Serrano-Sánchez, M. Gharsallah, N. M. Nemes, N. Biskup, M. Varela, J. L. Martínez, M. T. Fernández-Díaz, and J. A. Alonso. Enhanced figure of merit in nanostructured $(\mathrm{Bi}, \mathrm{Sb})_{2} \mathrm{Te}_{3}$ with optimized composition, prepared by a straightforward arc-melting procedure. Sci. Rep., 7:6277, 2017.

[116] R. Venkatasubramanian. Lattice thermal conductivity reduction and phonon localizationlike behavior in superlattice structures. Phys. Rev. B, 61:3091-3097, 2000.

[117] A.-L. Hansen, T. Dankwort, M. Winkler, J. Ditto, D. C. Johnson, J. D. König, K. Bartholomé, L. Kienle, and W. Bensch. Synthesis and Thermal Instability of High-Quality $\mathrm{Bi}_{2} \mathrm{Te}_{3} / \mathrm{Sb}_{2} \mathrm{Te}_{3}$ Superlattice Thin Film Thermoelectrics. Chem. Mater., 26:6518-6522, 2014. 
[118] R. Venkatasubramanian, T. Colpitts, B. O'Quinn, S. Liu, N. El-Masry, and M. Lamvik. Low-temperature organometallic epitaxy and its application to superlattice structures in thermoelectrics. Appl. Phys. Lett., 75:1104-1106, 1999.

[119] O. M. Corbino. Periodische Widerstandsänderungen feiner Metallfäden, die durch Wechselströme zum Glühen gebracht werden, sowie Ableitung ihrer thermischen Eigenschaften bei hoher Temperatur. Phys. Z., 12:292-295, 1911.

[120] D. G. Cahill. Thermal conductivity measurement from 30 to $750 \mathrm{~K}$ : the $3 \omega$ method. Rev. Sci. Instrum., 61:802-808, 1990.

[121] D. G. Cahill, M. Katiyar, and J. R. Abelson. Thermal conductivity of a-Si:H thin films. Phys. Rev. B, 50:6077-6081, 1994.

[122] H. S. Carslaw and J. C. Jaeger. Conduction of Heat in Solids. Oxford University Press, 2nd edition, 1946.

[123] P. Thiessen. 3w-method for the thermal characterisation of complex perovskite oxide superlattices. Master thesis, Georg-August-Universität Göttingen, 2016.

[124] T. Borca-Tasciuc, A. R. Kumar, and G. Chen. Data reduction in $3 \omega$ method for thin-film thermal conductivity determination. Rev. Sci. Instrum., 72:2139-2147, 2001.

[125] T. Tong and A. Majumdar. Reexamining the 3-omega technique for thin film thermal characterization. Rev. Sci. Instrum., 77:104902, 2006.

[126] D. A. Ditmars, S. Ishihara, S. S. Chang, and G. Bernstein. Enthalpy and HeatCapacity Standard Reference Material: Synthetic Sapphire $\left(\alpha-\mathrm{Al}_{2} \mathrm{O}_{3}\right)$ from 10 to 2250 K. Journal of Research of the National Bureau of Standards, 87:159-163, 1982.

[127] J. Lindner. Verfication and Optimization of an Advanced 3w-System for Measurement of Thermal Conductivity. Bachelor thesis, Georg-August-Universität Göttingen, 2014.

[128] G. Arend. Preparation and Analysis of Insulating Layers for the 3w-Method. Bachelor thesis, Georg-August-Universität Göttingen, 2016.

[129] S. A. Wiedigen. Thermische Leitfähigkeitsmessungen an Perowskit-Oxiden mittels der 3w-Methode. Diploma thesis, Georg-August-Universität Göttingen, 2010.

[130] C. E. Raudzis. Anwendung und Erweiterung der $3 \omega$ Methode zur Charakterisierung komplexer Mehrschichtsysteme in der Mikrosystemtechnik. Der Andere Verlag, 2007.

[131] M. Y. Ali, W. Hung, and F. Yongqi. A Review of Focused Ion Beam Sputtering. International Journal of Precision Engineering and Manufacturing, 11:157-170, 2010.

[132] D. B. Williams and C. B. Carter. Transmission Electron Microscopy. Springer US, 2nd edition, 2009. 
[133] R. Erni. Aberration-Corrected Imaging in Transmission Electron Microscopy. Imperial College Press, 2010.

[134] H. Sawada, T. Tomita, M. Naruse, T. Honda, P. Hambridge, P. Hartel, M. Haider, C. Hetherington, R. Doole, A. Kirkland, J. Hutchison, J. Titchmarsh, and D. Cockayne. Experimental evaluation of a spherical aberration-corrected TEM and STEM. J. Electron Microsc., 54:119-121, 2005.

[135] D. J. Smith. Ultimate resolution in the electron microscope? Mater. Today, 11:30$38,2008$.

[136] L. Wang, Z. Tan, S. Meng, A. Druzhinina, R. A. Varushchenko, and G. Li. Heat capacity enhancement and thermodynamic properties of nanostructured amorphous $\mathrm{SiO}_{2}$. J. Non-Cryst. Solids, 296:139-142, 2001.

[137] G. Bendt, S. Gassa, F. Rieger, C. Jooss, and S. Schulz. Low-temperature MOCVD deposition of $\mathrm{Bi}_{2} \mathrm{Te}_{3}$ thin films using $\mathrm{Et}_{2} \mathrm{BiTeEt}$ as single source precursor. J. Cryst. Growth, 490:77-83, 2018. 



\section{Acknowledgement}

During my work at the Institute of Material Physics, I have been supported by many colleagues on both the scientific and the technical level. Therefore, I want to express my deep gratitude to all members of the IMP and in particular to those of the working group Jooß.

At first I want to thank Prof. Dr. Christian Jooß for the possibility of carrying out fundamental research and of writing my doctoral thesis in an open and appreciative work environment. I have valued the profound goal-oriented discussions which always promoted my research and expanded my scientific horizon.

I like to thank Prof. Dr. Vasily Moshnyaga for not only being co-referee of my thesis, but also for the trusting and fruitful scientific cooperation.

Thanks to Dr. Vladimir Roddatis who got the maximum out of our sessions at the TITAN providing impressive high-resolution images and in-depth insights into tiniest structures. Jonas Lindner did a great job by writing and improving the $3 \omega$ measurement program. Patrick Thiessen has to be mentioned for his magnificent dedication to the $3 \omega$ analysis and for our numerous profound discussions. Mike Kanbach spent a lot of time in the clean room establishing resist structures of excellent reliability, which formed the basis for the precise $3 \omega$ measurements shown in the present thesis.

I would like to especially thank our DFG project partners at the Institute of Inorganic Chemistry at the University of Duisburg-Essen, Prof. Dr. Stephan Schulz, Dr. Georg Bendt and last but not least Kevin Kaiser for great interdisciplinary and pleasant cooperation. Thanks to the untiring efforts of Kevin Kaiser, it even became possible to carry out the presented measurements at films of exceptional quality.

At this point I want to express my gratitude to the DFG for funding my work.

Explicitly, I want to thank the technical staff of the IMP enabling the smooth running of diverse machines I have used. The electronic workshop laid the foundation for the $3 \omega$ setup by building customized components. And the strong commitment of Michael Malchow, Matthias Hahn and Volker Raddisch to sputter devices, electron microscopes and the FIB, enabled my work to be possible in this way.

Countless conversations with colleagues on scientific topics and beyond helped me to advance and made my work more comfortable. To name only a few, I really enjoyed the discussions with Emanuel Ronge, Jonas Lindner and Leif Kochanneck.

Finally, my sincere thanks go to friends and family for their encouragement, in particular to my sister Ruth Rieger and my parents Judith and Norbert Rieger.

Most especially I thank my girlfriend Isabell Brendel for providing backing and distraction all the time and for the extensive proofreading during the last days. 